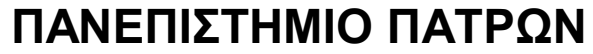

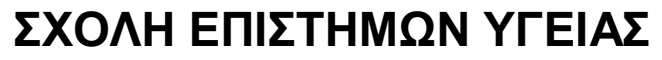

TMHMA IATPIKH $\Sigma$

\author{
ПАӨО^ОГІКН КАINIKH-PЕYMАТО^ОГІКО TМНМА

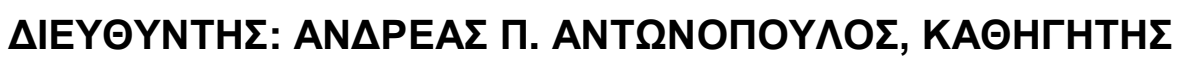

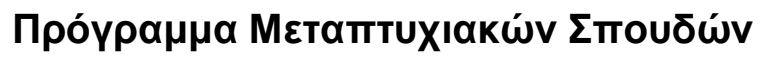

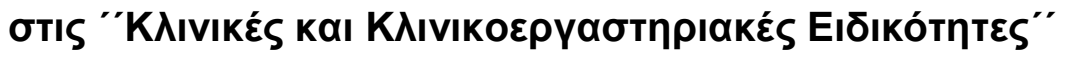

\begin{abstract}
$\Delta$ ІठакторІки́ $\Delta$ Іатрıßи́

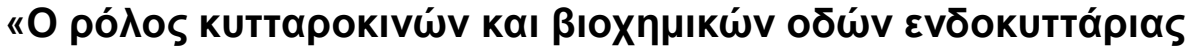

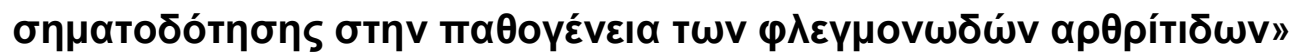

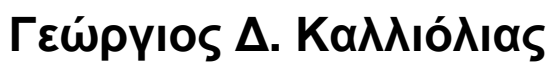

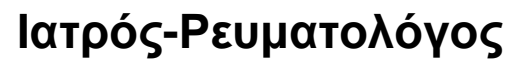

ПАТРА 2009 


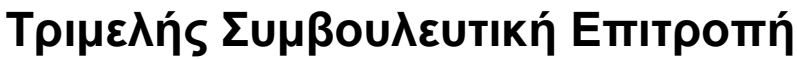

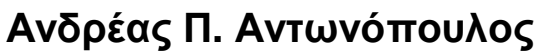

KaӨnүптńs

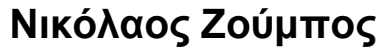

KaӨnүптńs

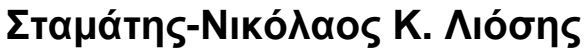

Eтíkoupos KaӨnүптńs

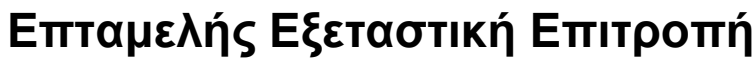

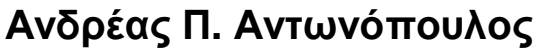 \\ KaӨnүптńs

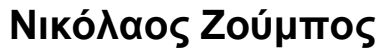 \\ KaӨnүฑтńs

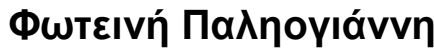 \\ КаӨпүท́трıа

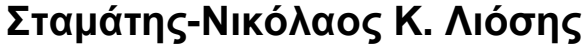 \\ Eтíkoupos KaӨnynтńs

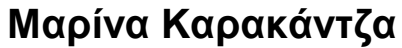 \\ Еті́коupп KaӨпүท́трıа

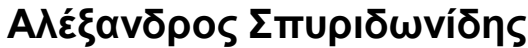 \\ Eтíkoupos KaӨnүптńs

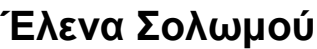 \\ \દ́ктораऽ
}




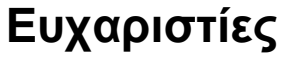

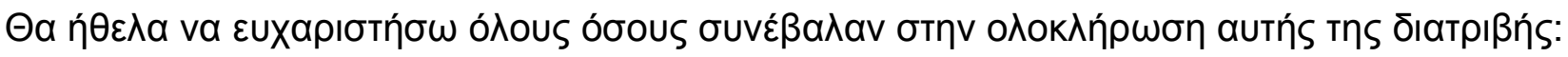

Tov Dr Lionel B. Ivashkiv (Professor of Medicine and Immunology, Weill Medical College of

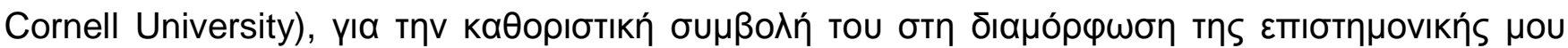

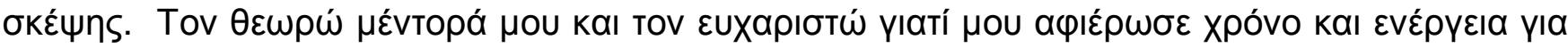

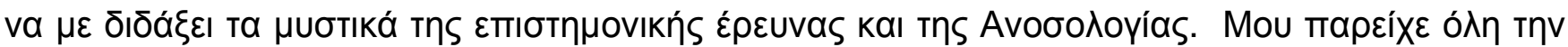

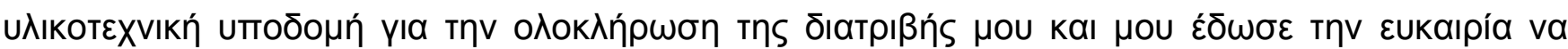

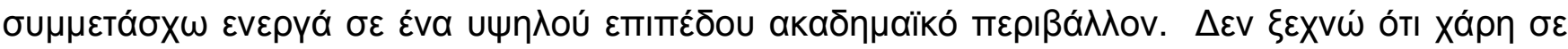

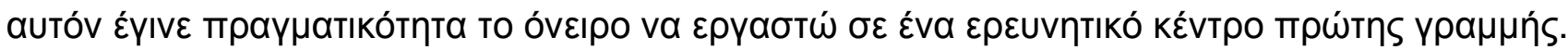

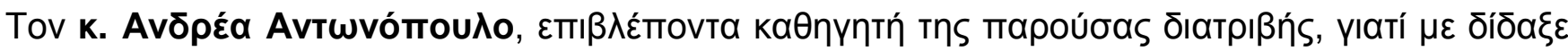

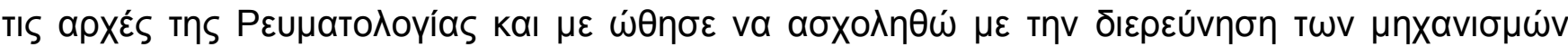

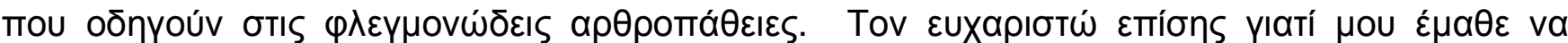

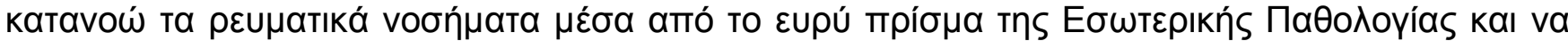

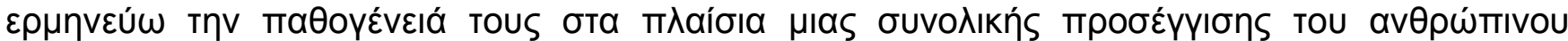
opyaviouoú.

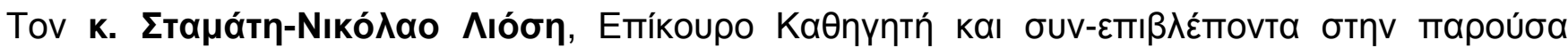

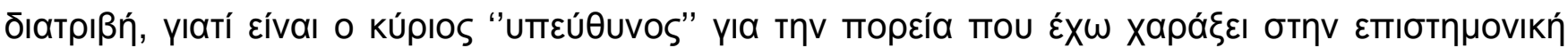

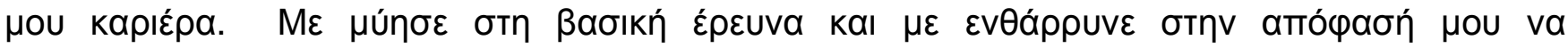

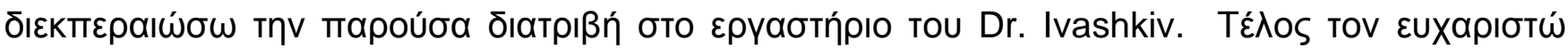

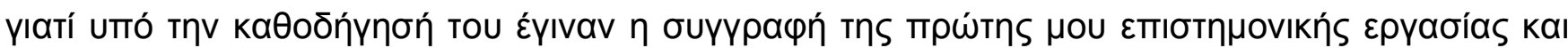




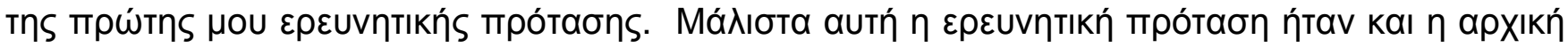

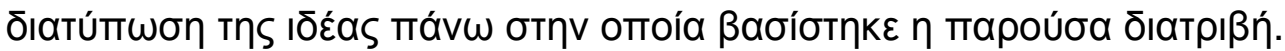

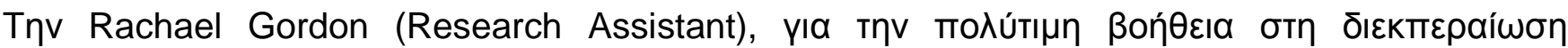

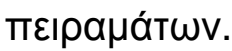

Tov Dr. Jong Dae Ji (Associate Professor, Division of Rheumatology, College of Medicine, Korea

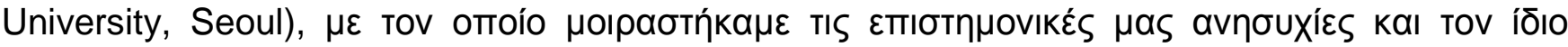
та́үүко үıа 2 xpóvıа.

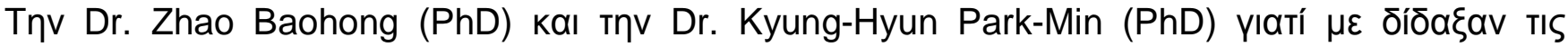

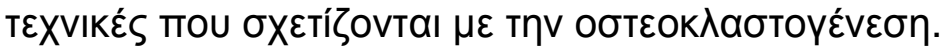

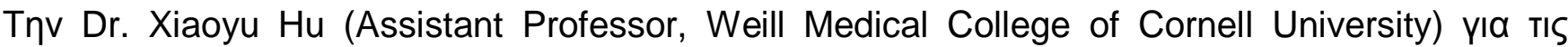

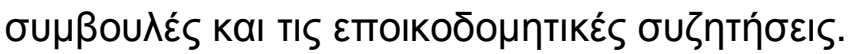

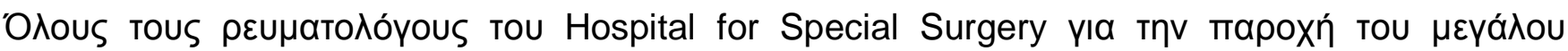

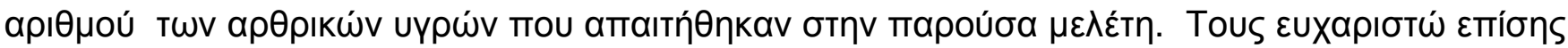

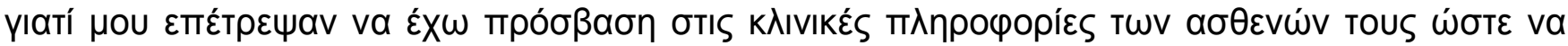

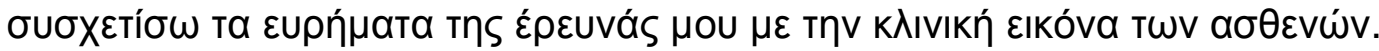

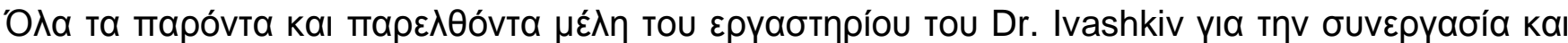

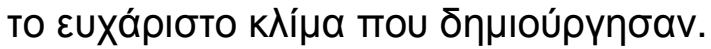




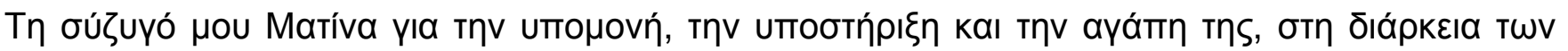

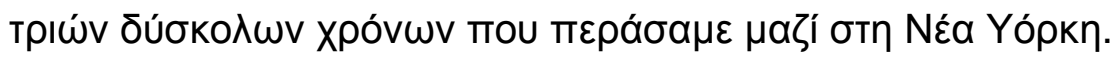

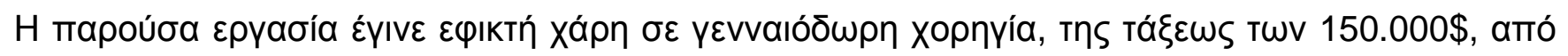
то Arthritis Foundation, New York Chapter (Stavros S. Niarchos International Fellowship

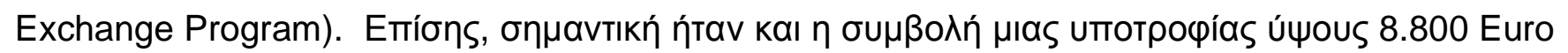

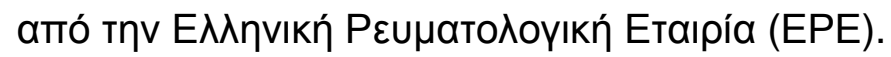




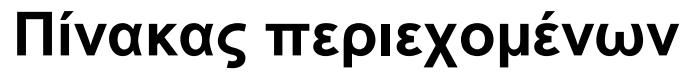

$\Sigma \varepsilon \lambda i ́ \delta \alpha$

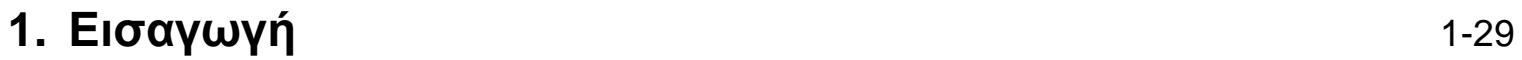

$\begin{array}{ll}\text { 1.1. PEYMATOEI } \Delta H \Sigma \text { APOPITISA } & \text { 2-17 }\end{array}$

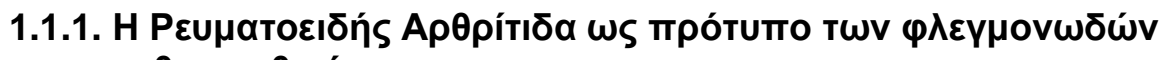
$\alpha \rho \theta \rho \circ \pi \alpha \theta \varepsilon ı \omega ́ v$.

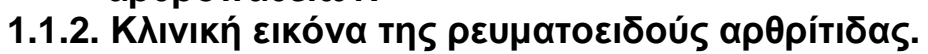

3-4

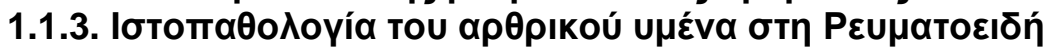

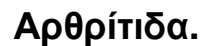

\section{5}

$6-17$

6

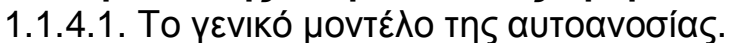

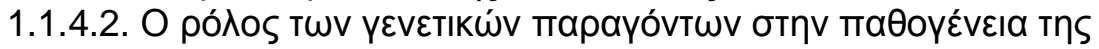

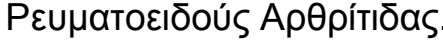

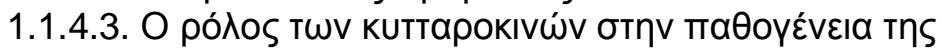

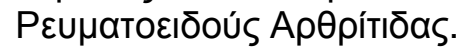

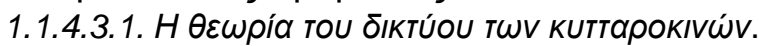

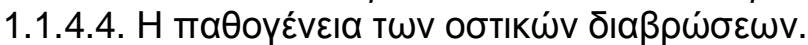

1.2. INTEP^EYKINH 27 (IL-27)

1.2.1. $\Delta$ ouń tnS IL-27.

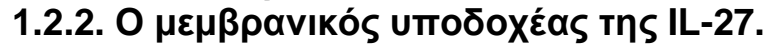

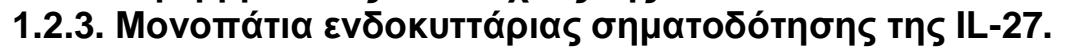

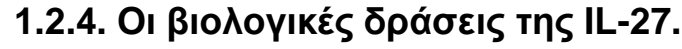

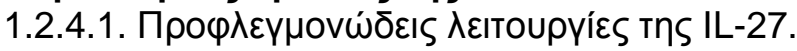

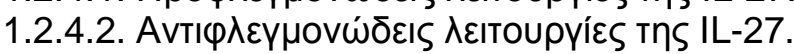

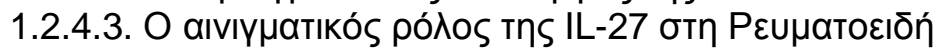
АрӨрі́тıठ́а.

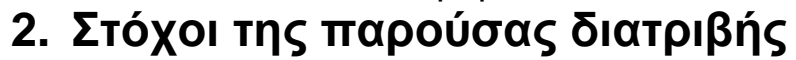

$7-8$

9-12

11-12

13-17

$18-29$

$18-19$

20-21

$22-23$

24-29

$24-25$

26-27

28-29

30-32

$33-42$

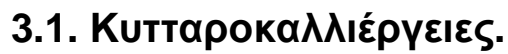

34-35

35

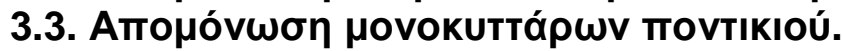

35-36

36-37

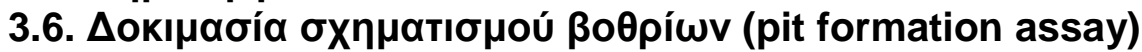

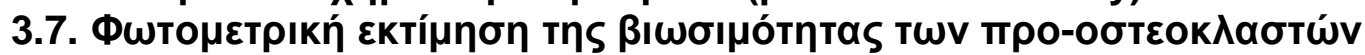
(MTT assay)

38

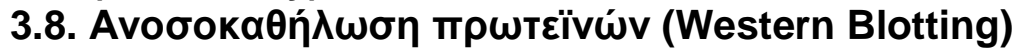

38-39

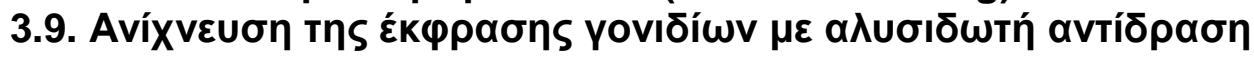

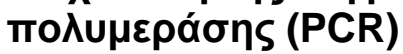

$39-42$

$43-89$

$44-59$

48-49

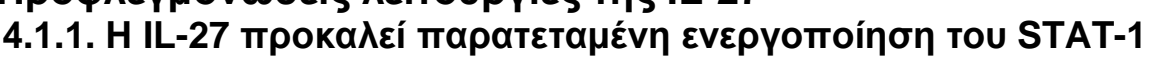

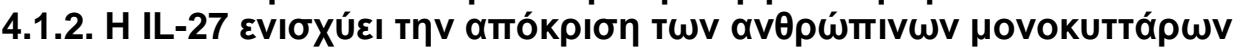

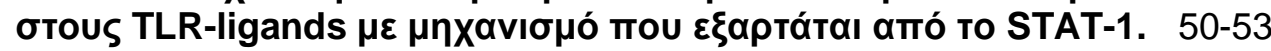




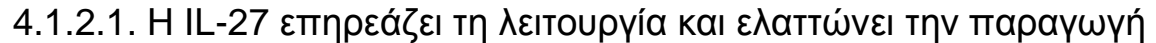 TnS IL-10}

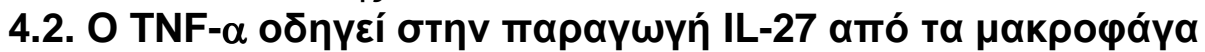

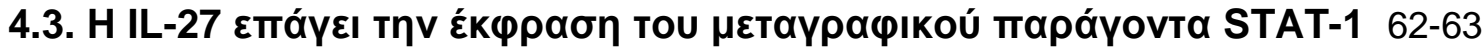

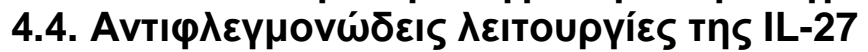

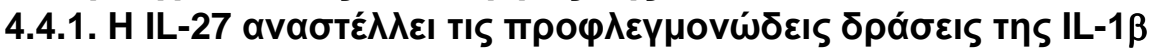

64-66

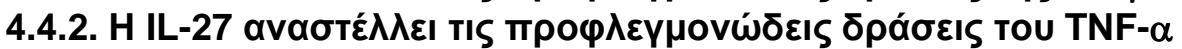

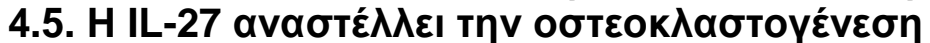

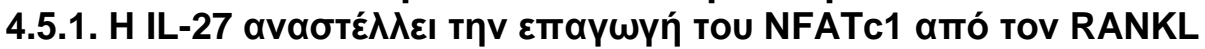

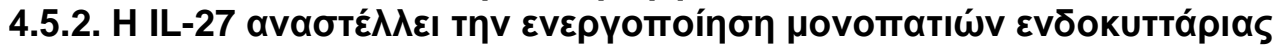

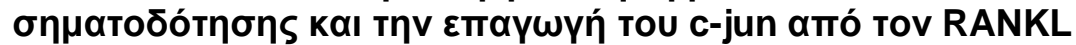

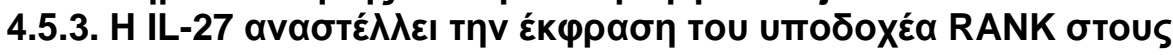

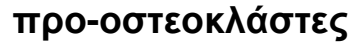

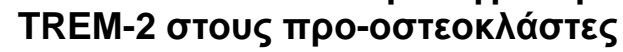

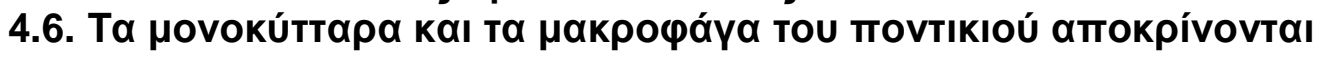

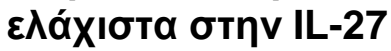

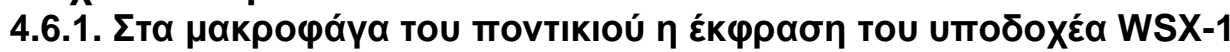

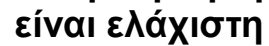

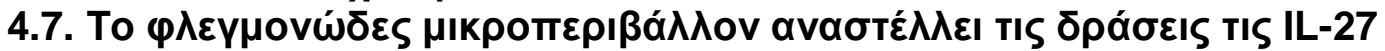

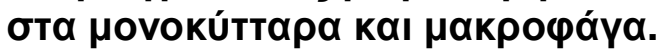

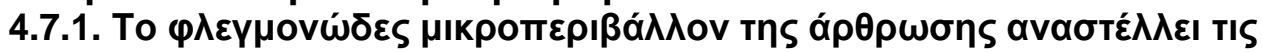

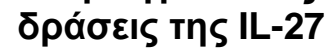

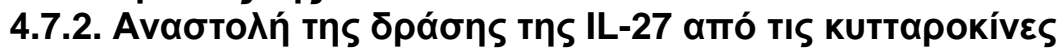
TNF- $\alpha$ KaI IL-1 $\beta$

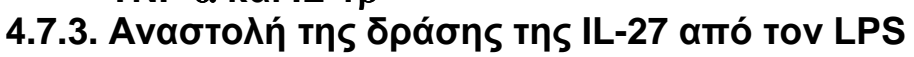

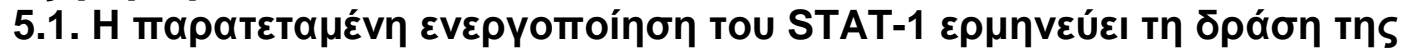

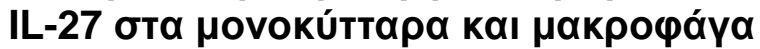

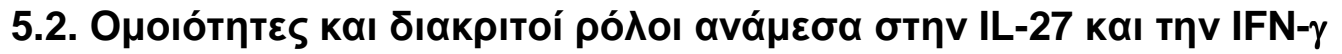

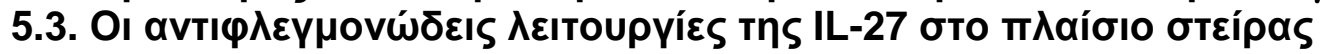
$\varphi \lambda \varepsilon Y \mu o v n ́ s$

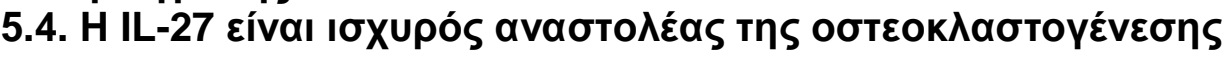

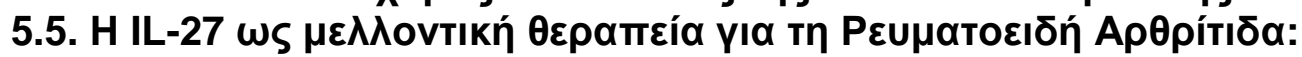

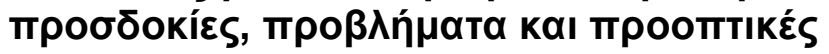

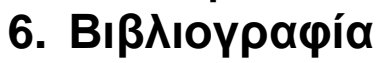

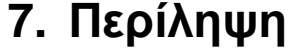

126-128

8. Summary

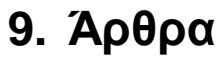




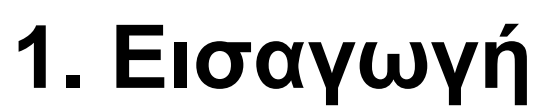




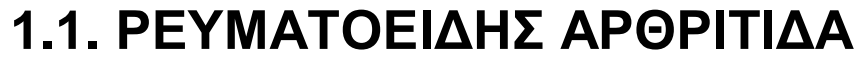

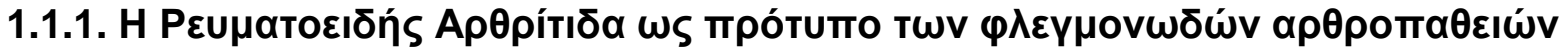

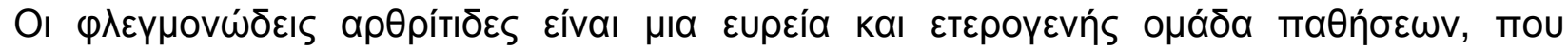

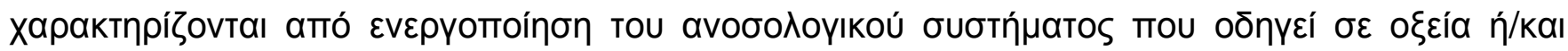

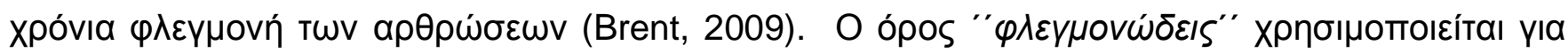

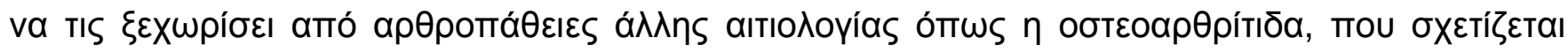

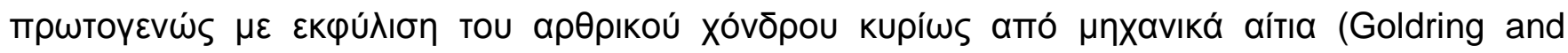

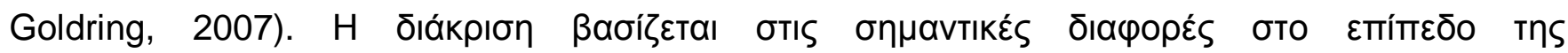

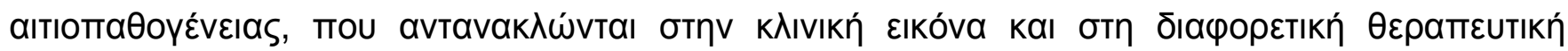
avтıнєти́тıоn (Berenbaum, 2008; Marino and McDonald, 1991; O'Dell, 2004; Schett, 2007). To

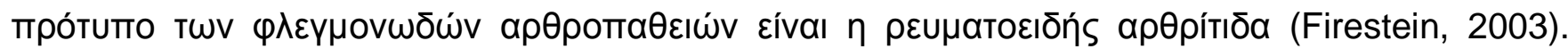

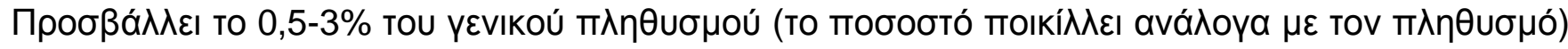

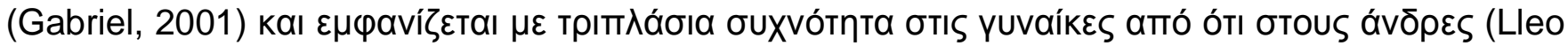
et al., 2008). 

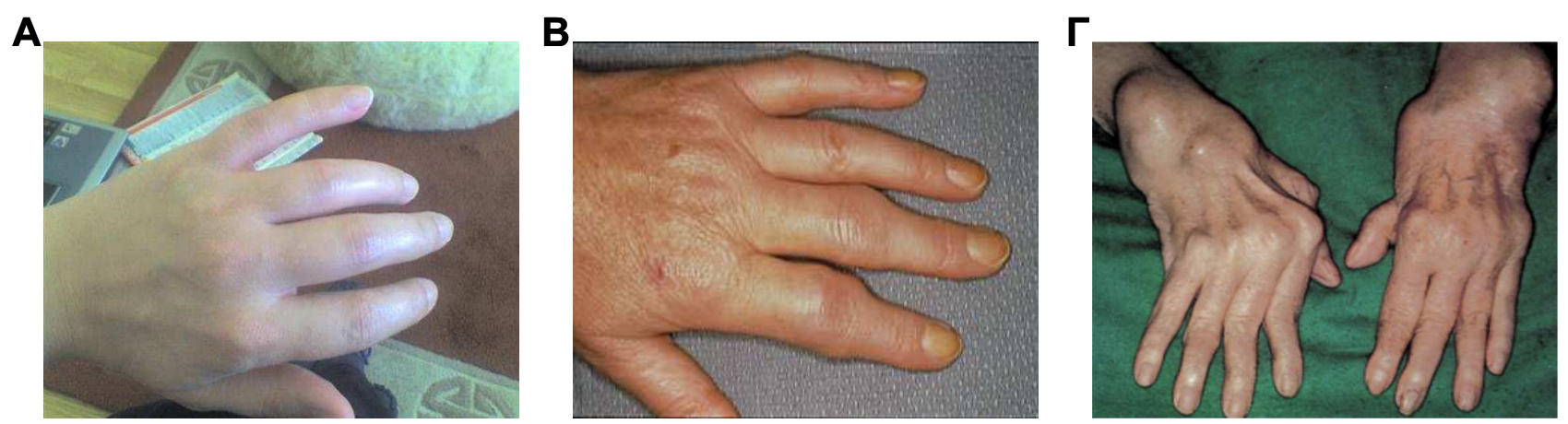

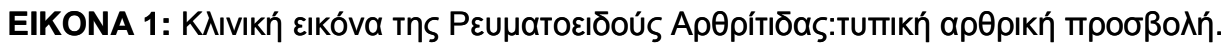

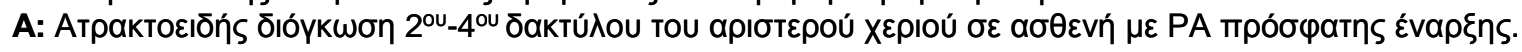

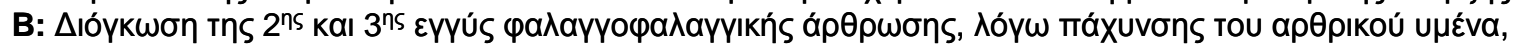

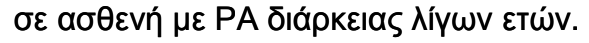

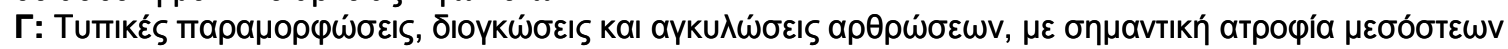

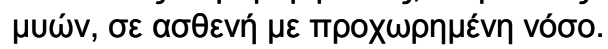

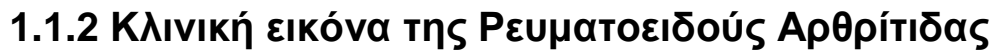

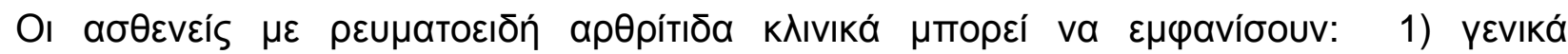

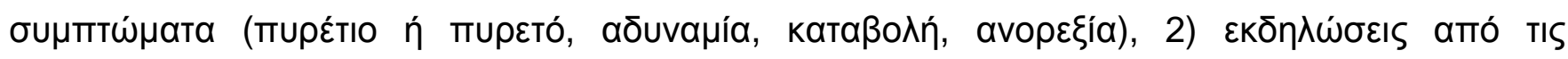

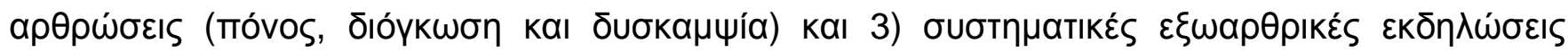

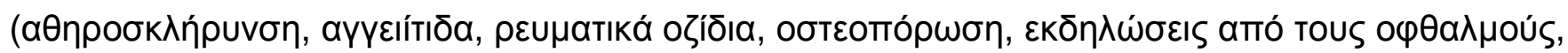

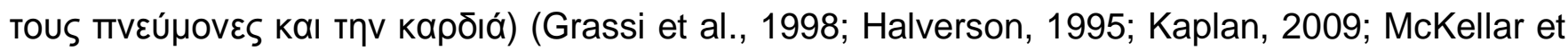

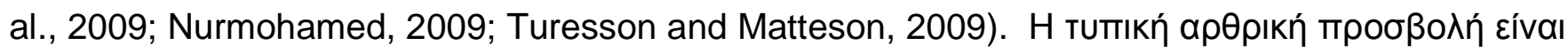

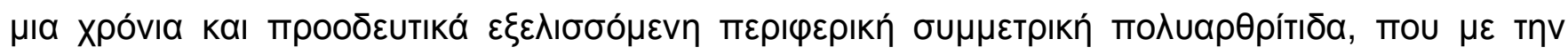

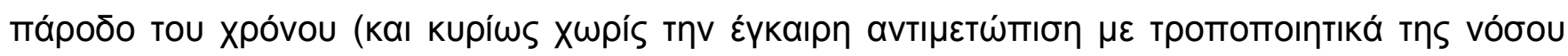

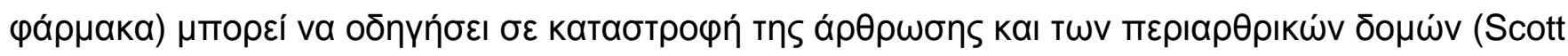

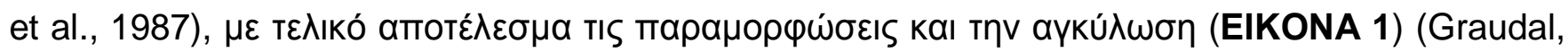

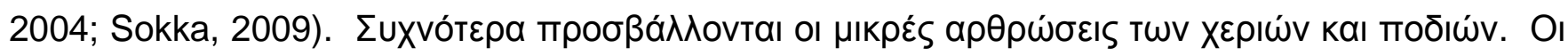

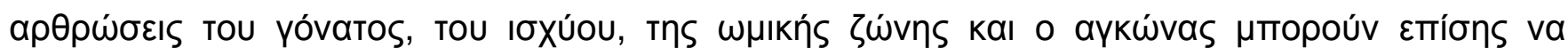

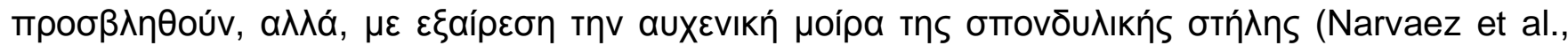

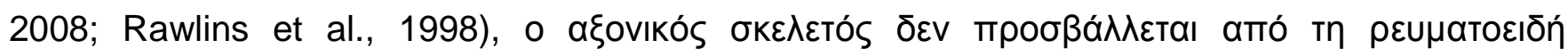




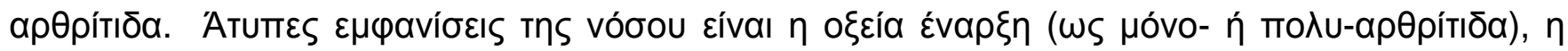

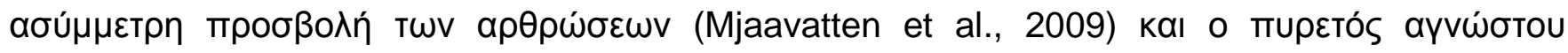
aıтı০^oyías (Revaz et al., 2006). 


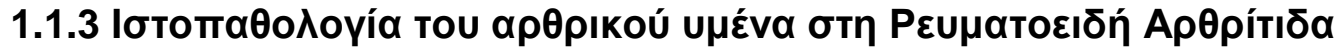

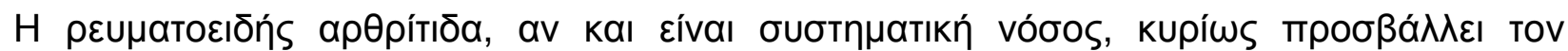

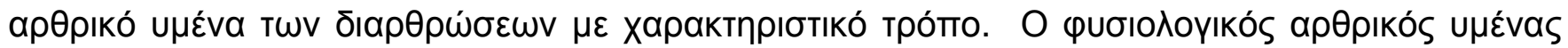

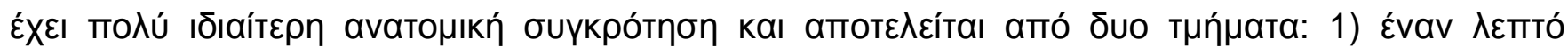

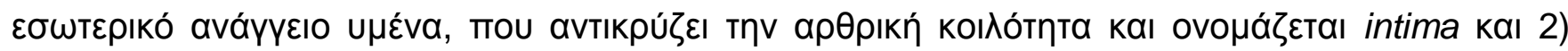

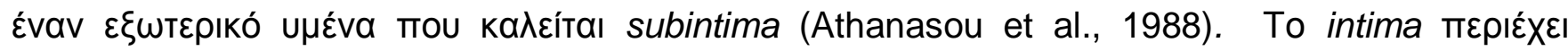

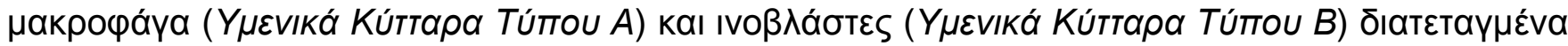

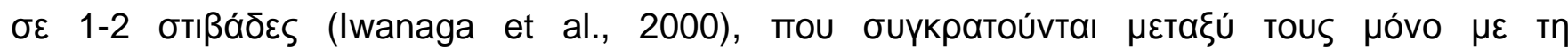

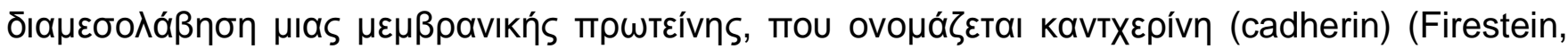

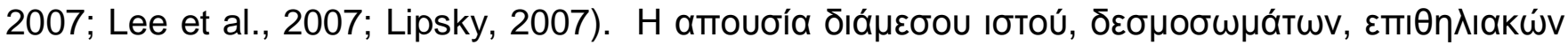

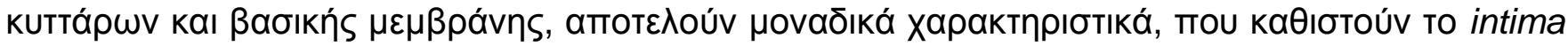

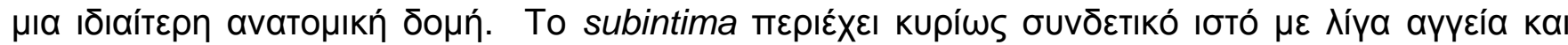

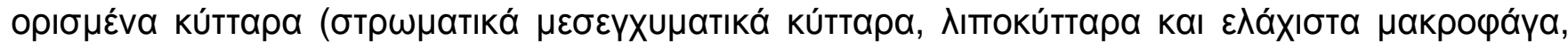

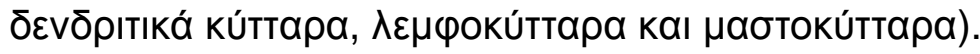

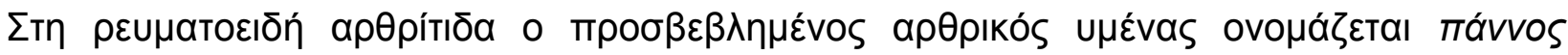

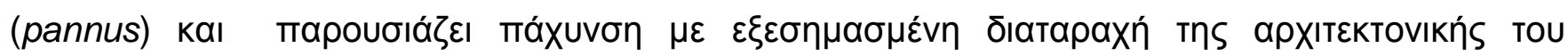
(Bresnihan et al., 2000; Hitchon and el-Gabalawy, 2003; van Oosterhout et al., 2008) kal

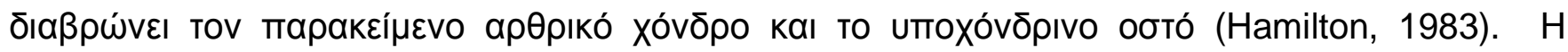

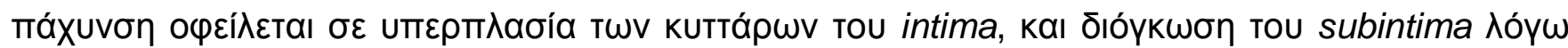

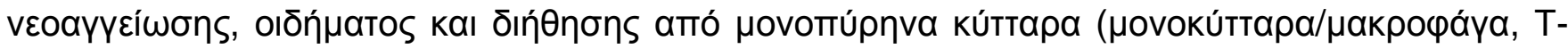

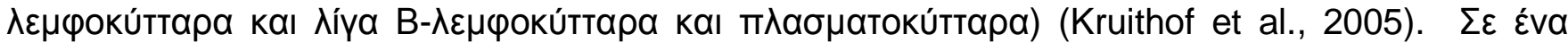

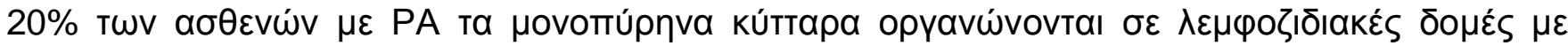

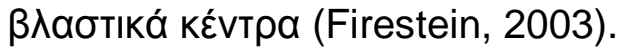




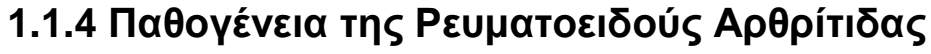

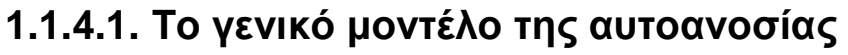

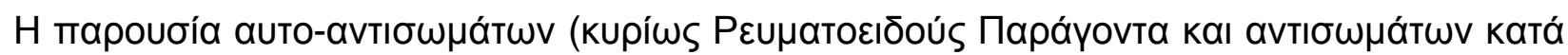

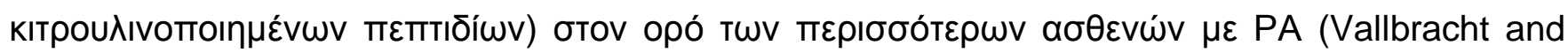

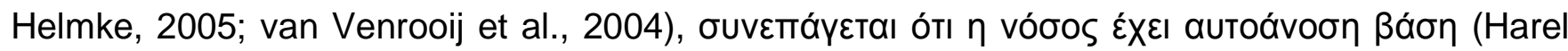

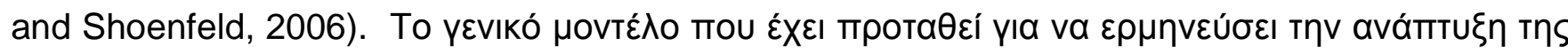

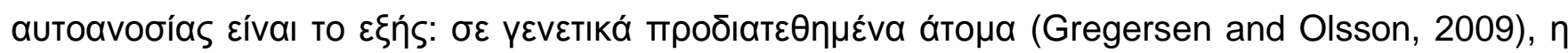

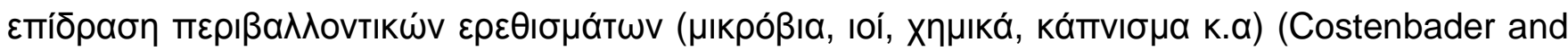

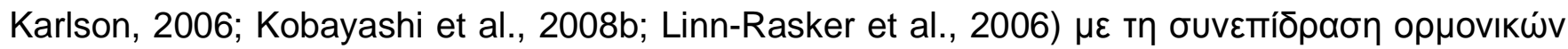

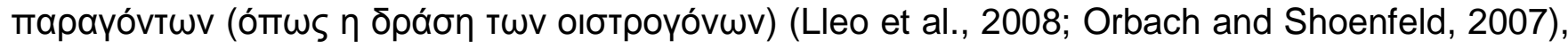

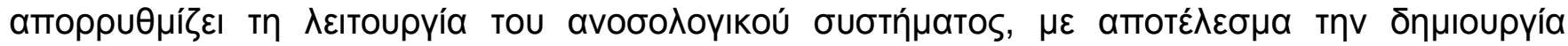

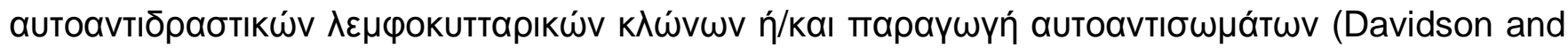
Diamond, 2001; Klareskog et al., 2006a; Klareskog et al., 2006b; Theofilopoulos, 1995a; Theofilopoulos, 1995b). 


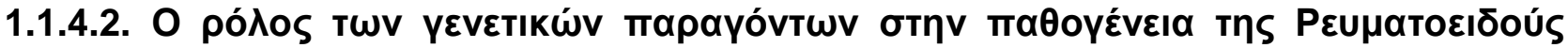

\section{ApӨpítıסаs}

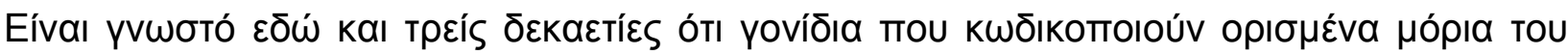

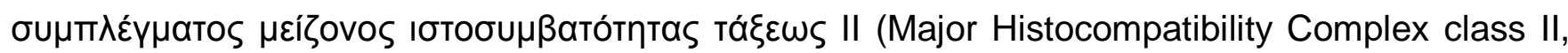

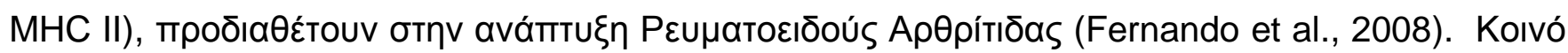

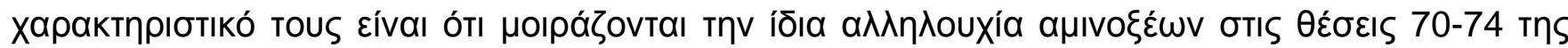

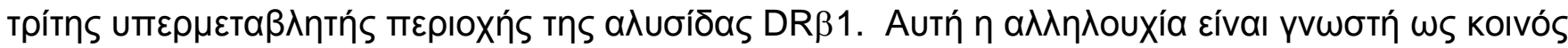

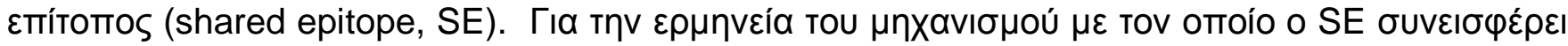

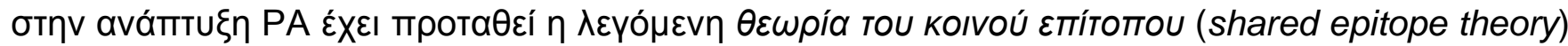

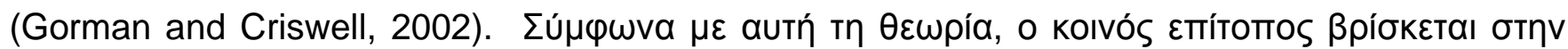

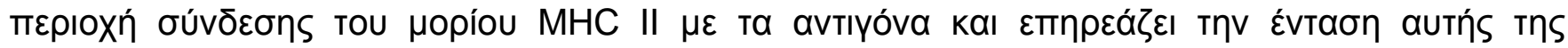

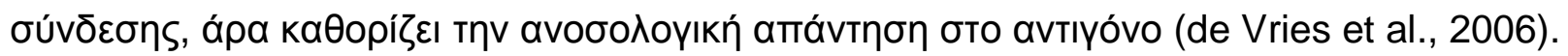

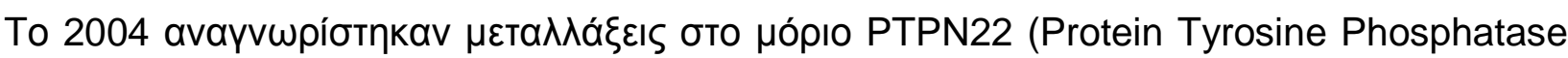

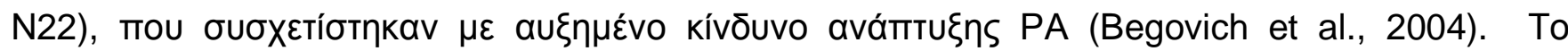

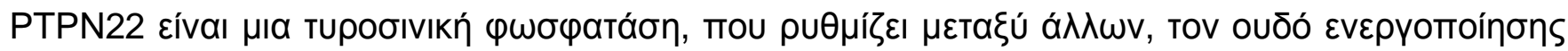

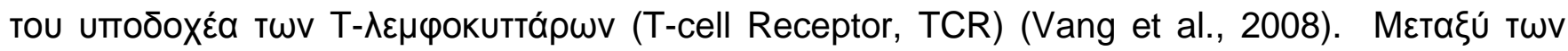

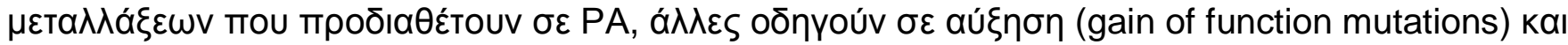

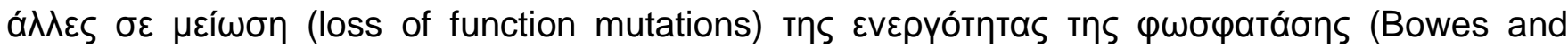

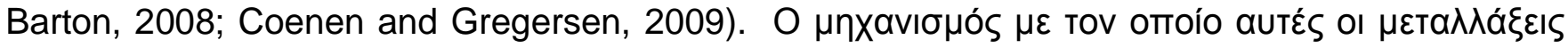

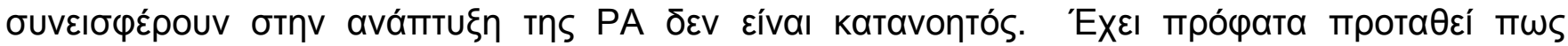

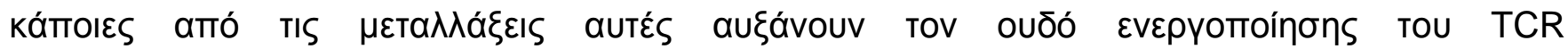

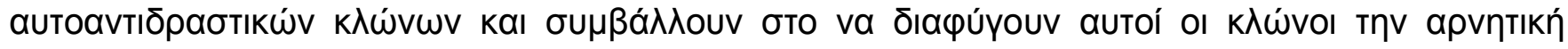

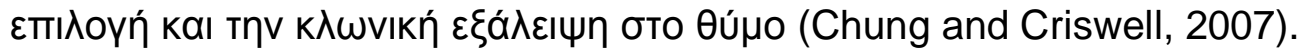




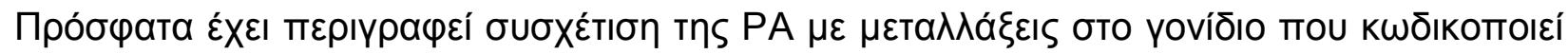

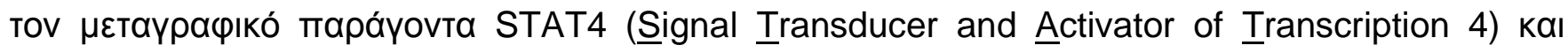

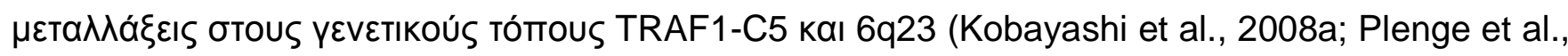

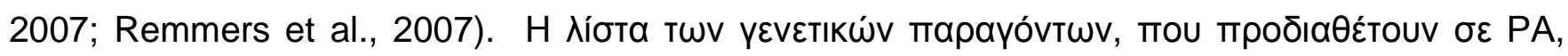

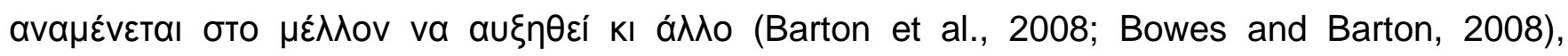

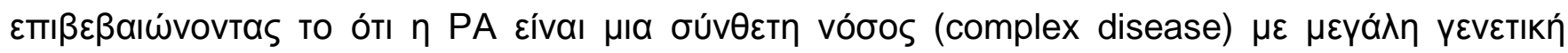

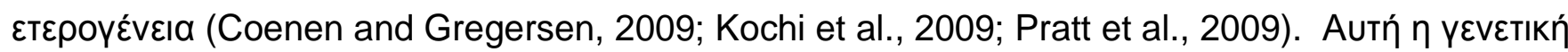

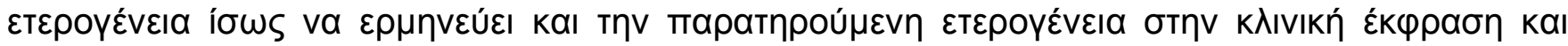

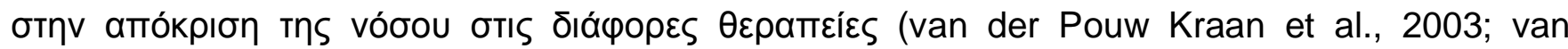
Oosterhout et al., 2008). 


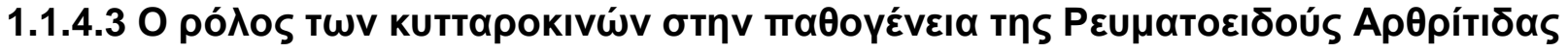

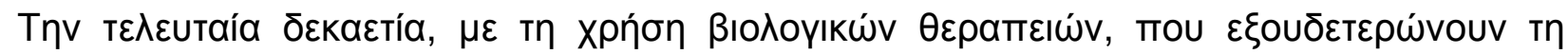

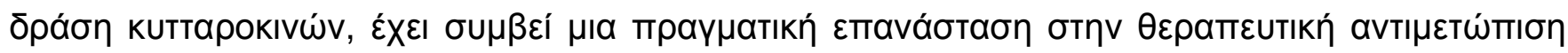

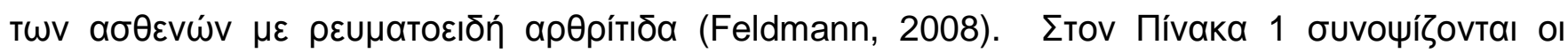

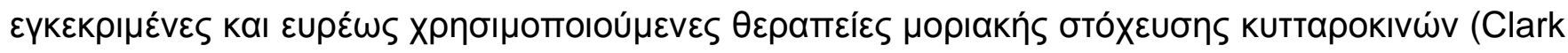
et al., 2004; Mertens and Singh, 2009; Scott and Kingsley, 2006; Taylor and Feldmann, 2009;

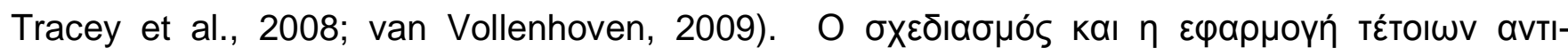

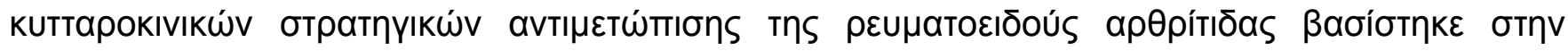

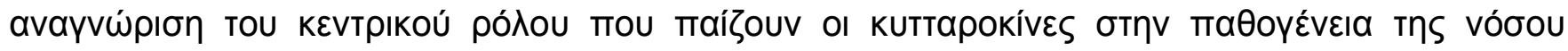
(Brennan and Mclnnes, 2008; Choy and Panayi, 2001; Feldmann, 2009; Feldmann et al., 2001; Gabay and Mclnnes, 2009).

\begin{tabular}{|c|c|}
\hline $\begin{array}{l}\text { ITOXEYOMENH } \\
\text { KYTTAPOKINH }\end{array}$ & 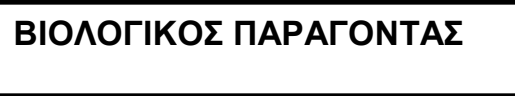 \\
\hline \multicolumn{2}{|l|}{ A) TNF- $\alpha$} \\
\hline & 1. Etanercept (Enbrel) \\
\hline & 2. Infliximab (Remicade) \\
\hline & 3. Adalimumab (Humira) \\
\hline & 4. Certolizumab Pegol (Cimzia) \\
\hline & 5. Golimumab (Simponi) \\
\hline \multicolumn{2}{|l|}{ B) IL-1 } \\
\hline & 1. Anakinra (Kineret) \\
\hline
\end{tabular}

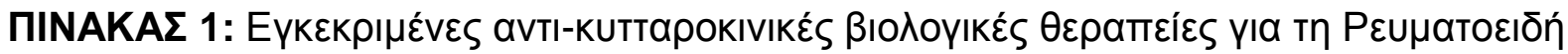
АрӨрі́тІठа. 


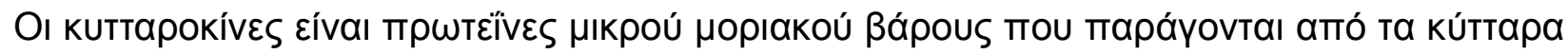

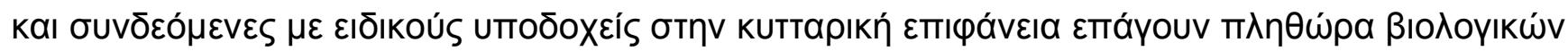

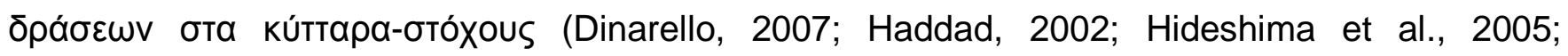

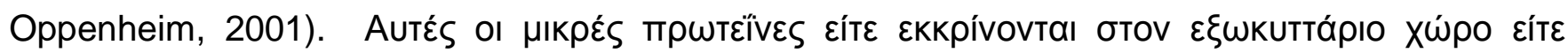

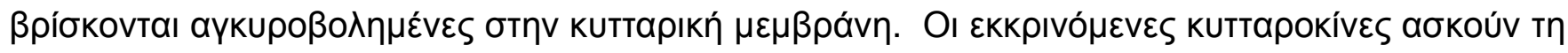

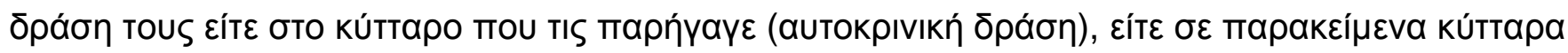

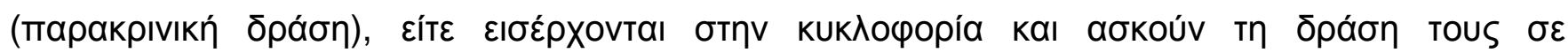

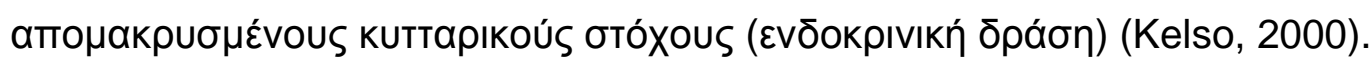




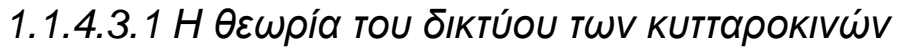

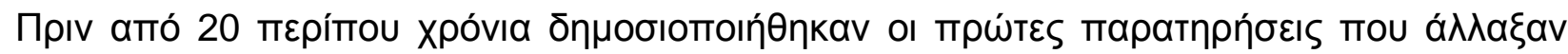

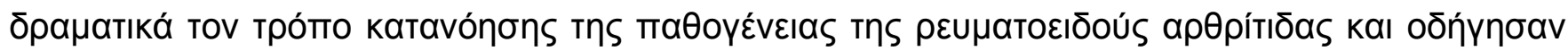

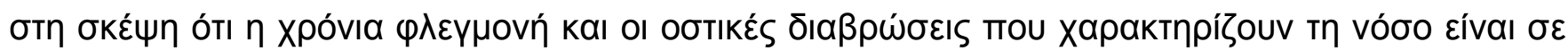

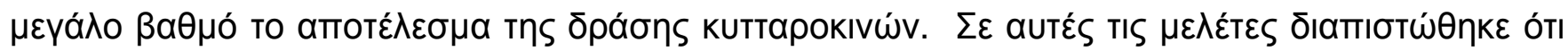

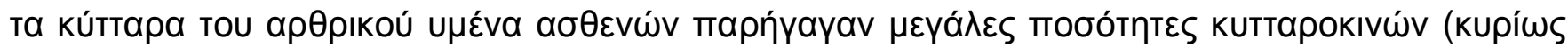

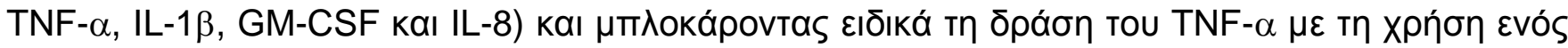

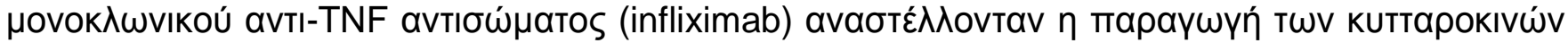
autúv (Brennan et al., 1989a; Brennan et al., 1989b; Haworth et al., 1991). A

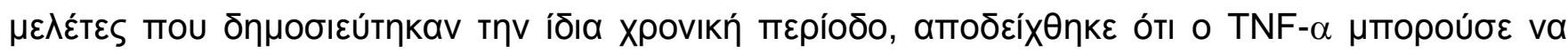

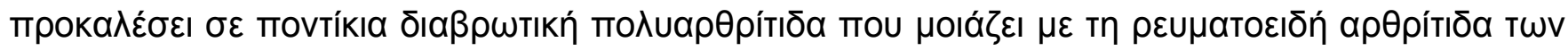

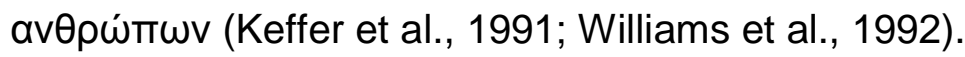

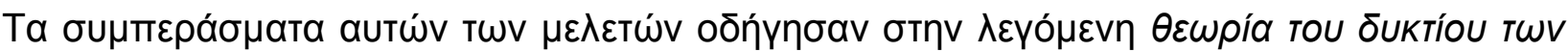

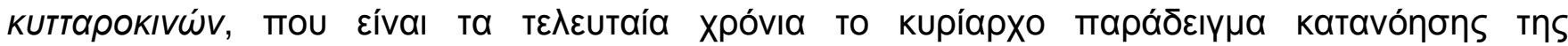

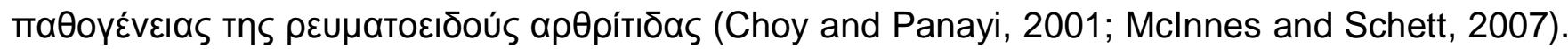

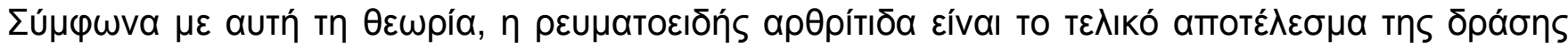

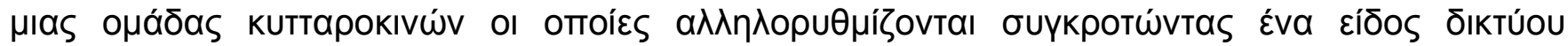

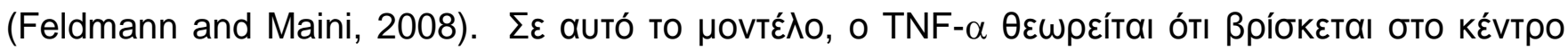

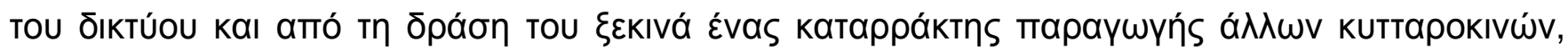

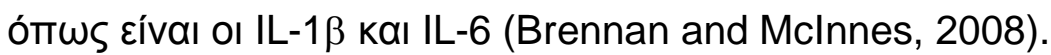

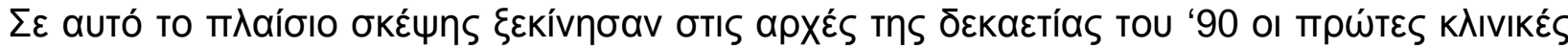

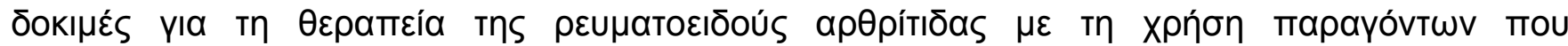

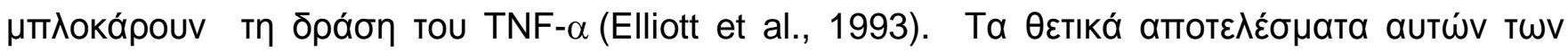




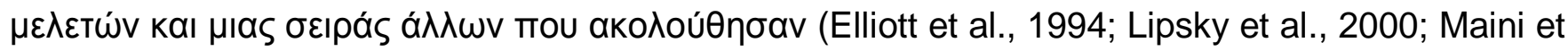

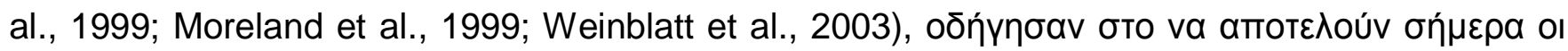

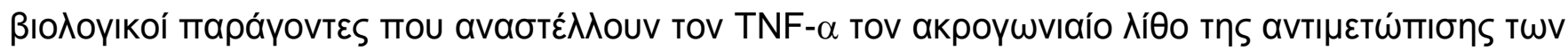

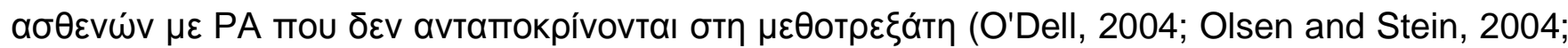

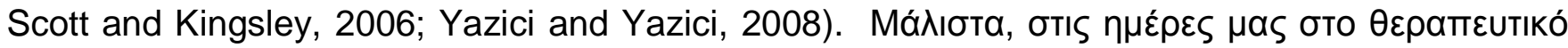

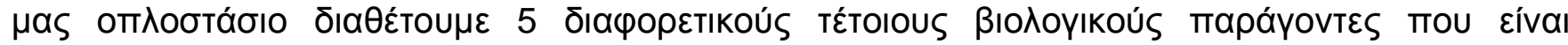

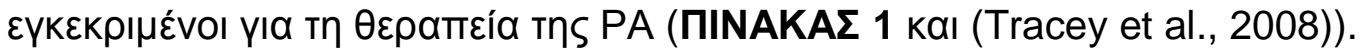

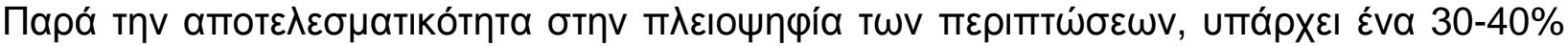

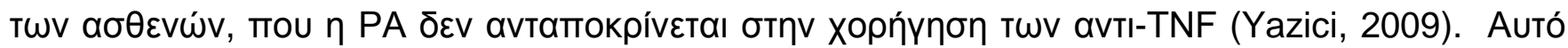

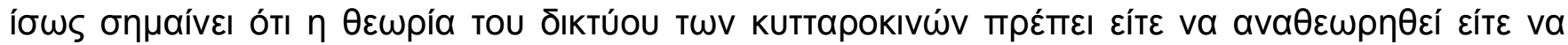

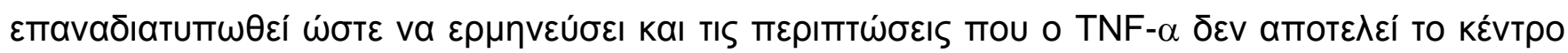

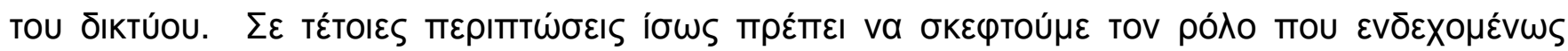

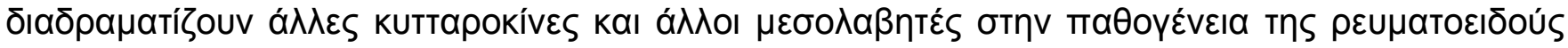
aрӨрі́тıбas (Gabay and Mclnnes, 2009). 


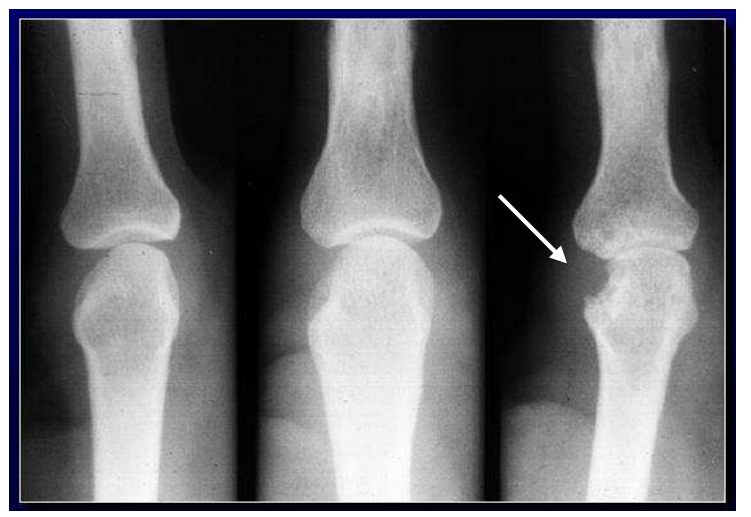

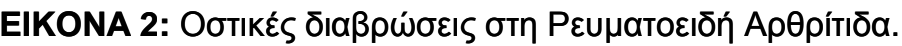

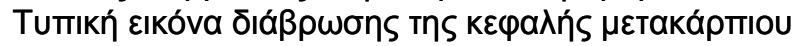

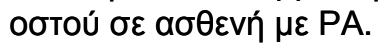

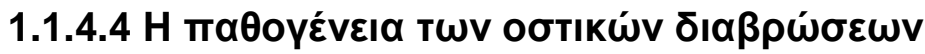

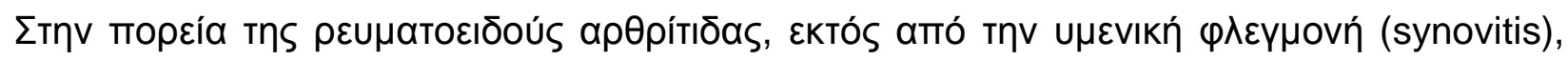

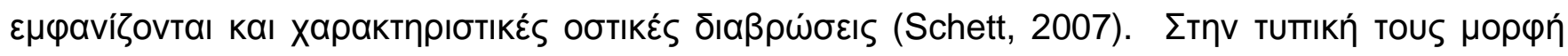

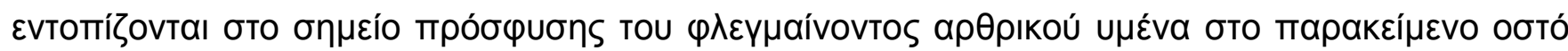

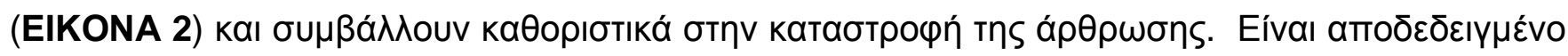

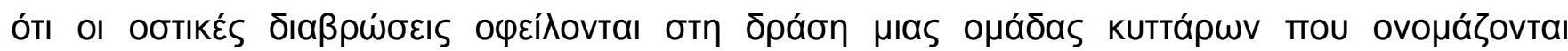

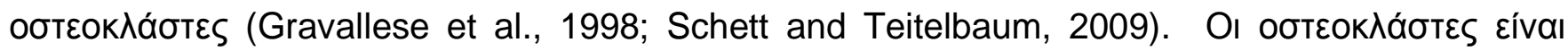

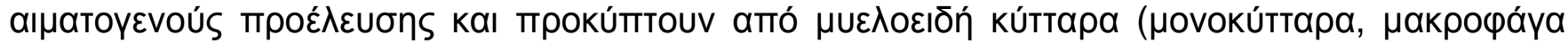

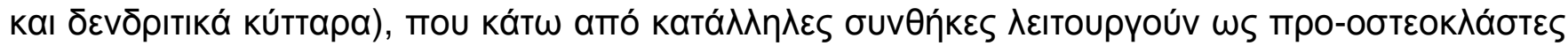

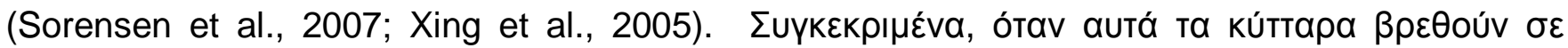

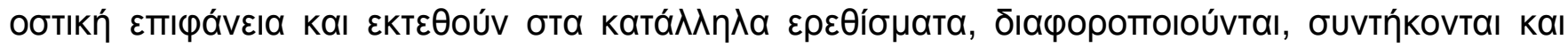

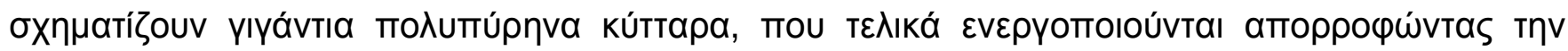

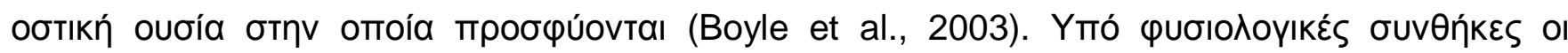

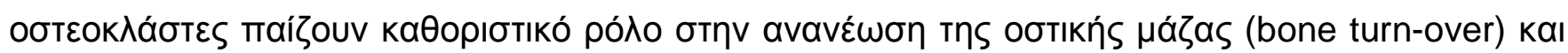

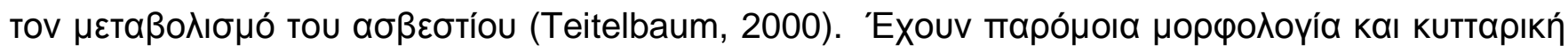

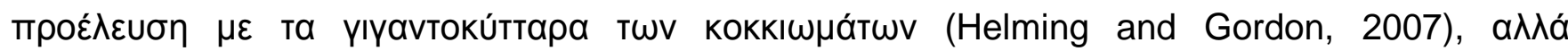

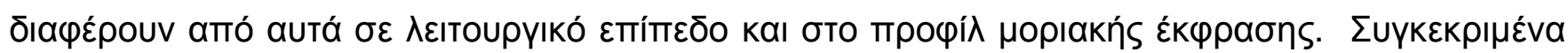




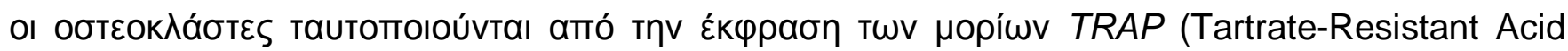
Phosphatese), Calcitonin Receptor, Cathepsin K kaı Integrinß3 (Boyle et al., 2003; Lee et al., 1995).

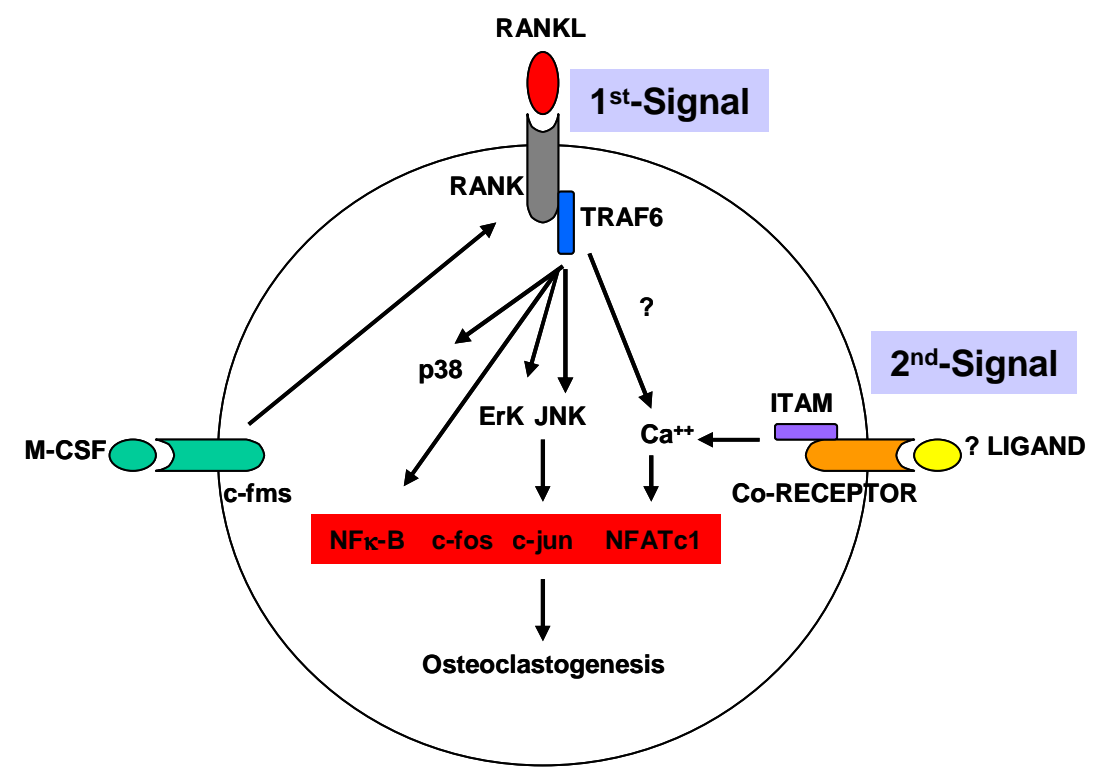

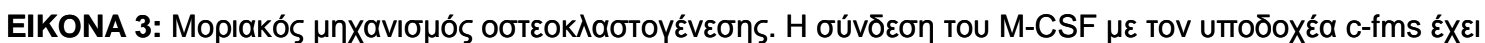

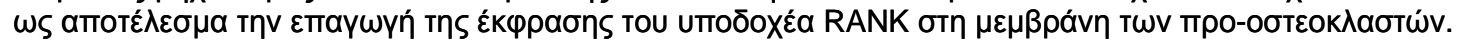

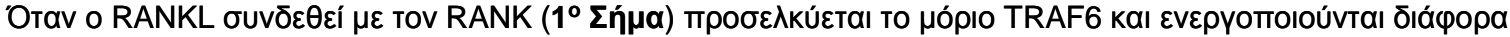

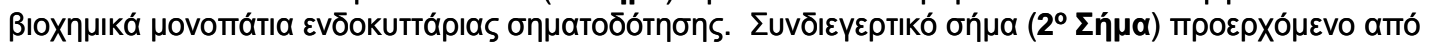

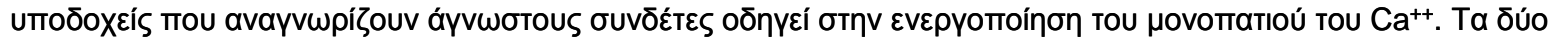

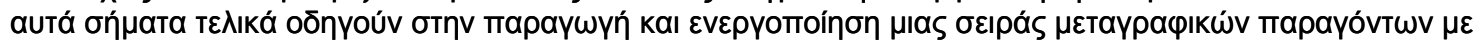

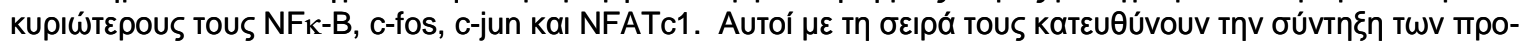

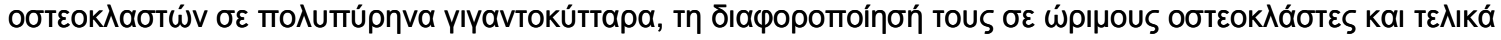

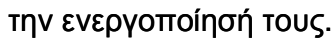

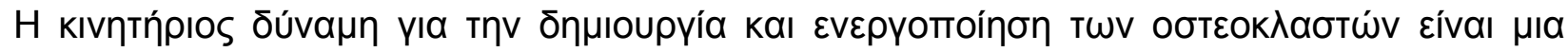

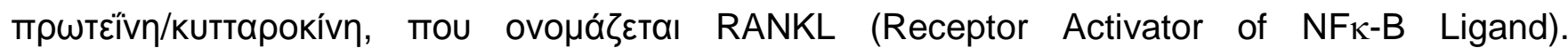

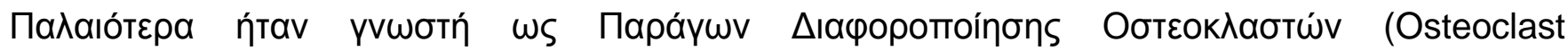

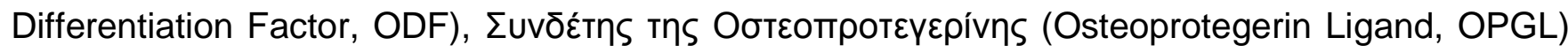
(Lacey et al., 1998) ń TRANCE (INF-

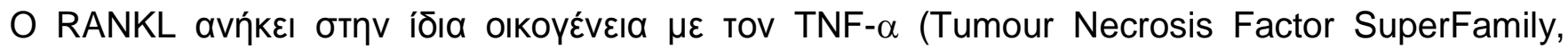

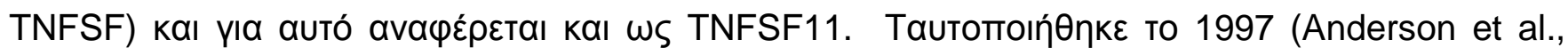




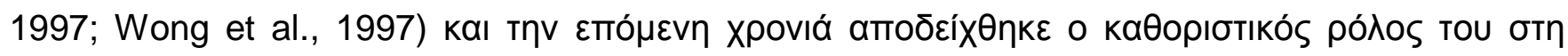

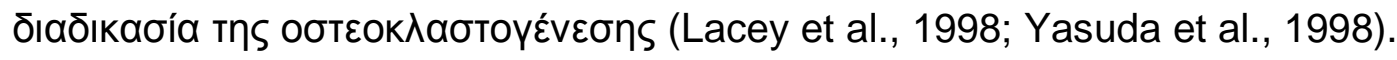

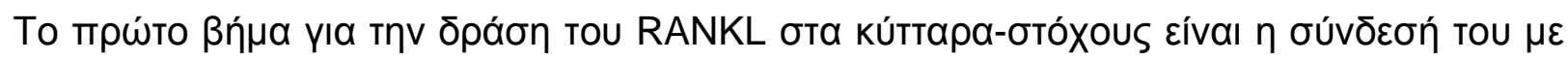

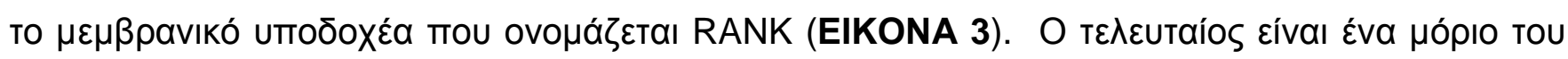

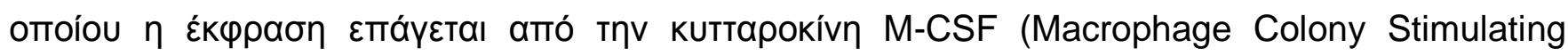

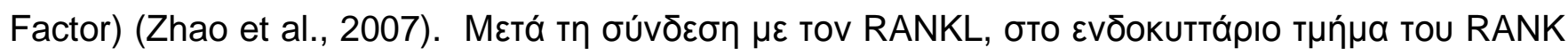

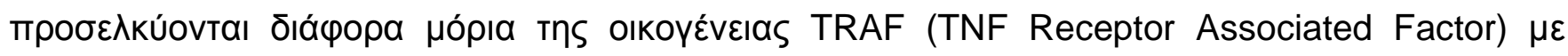
кupıóтєро то TRAF6 (Galibert et al., 1998; Gohda et al., 2005; Kobayashi et al., 2001; Mizukami

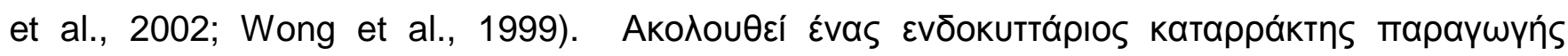

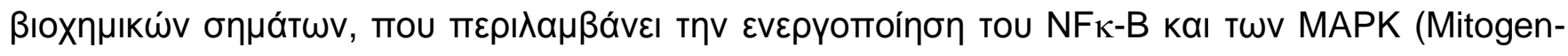

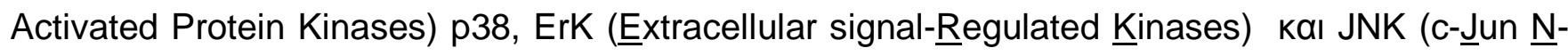
terminal Kinases) (Takayanagi, 2007a; Wada et al., 2006).

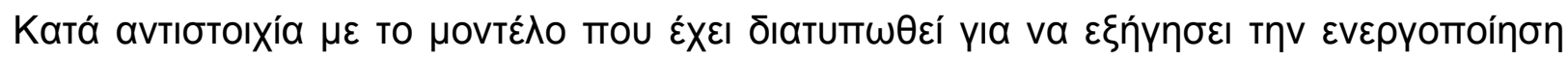

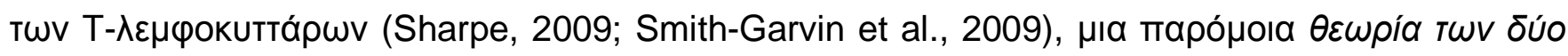

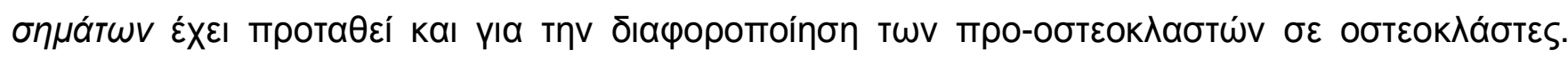

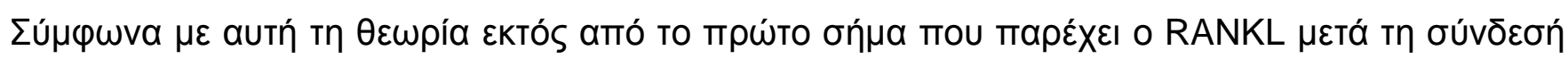

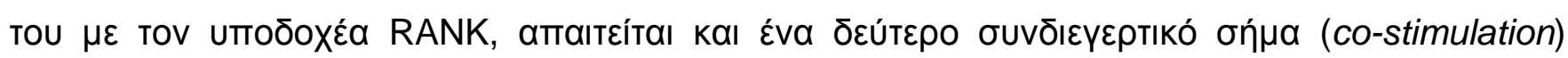

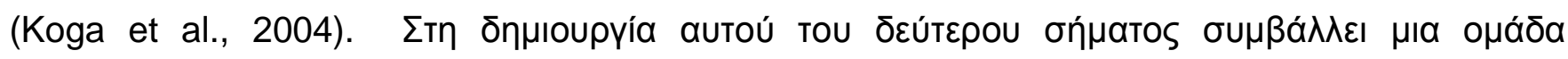

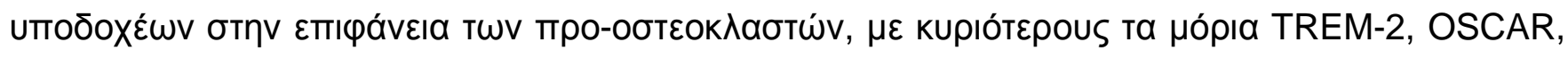
SIRPß1 kaı ILT7 (Humphrey et al., 2006; Matsuo and Irie, 2008; Paloneva et al., 2003). 'Otav ol

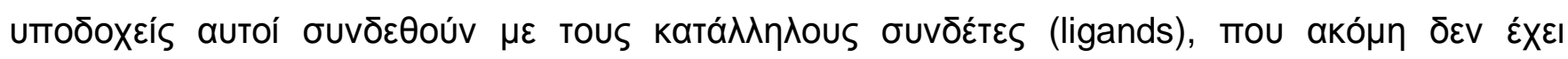

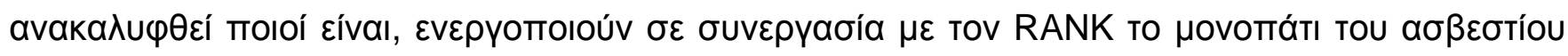

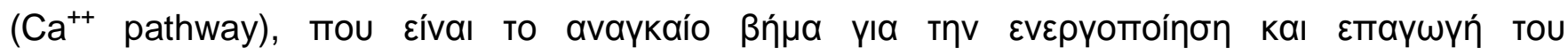




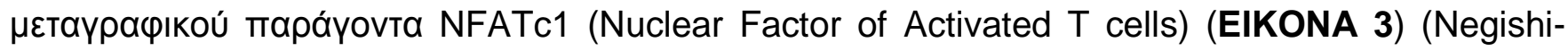

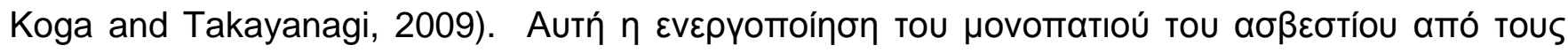

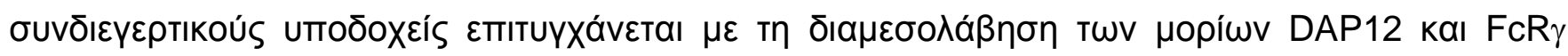

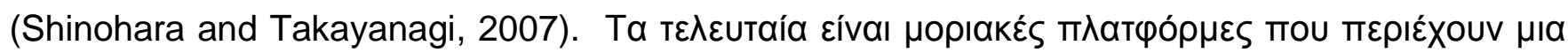
a

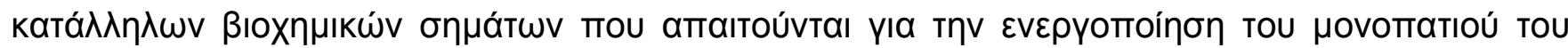

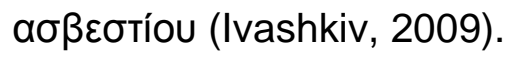

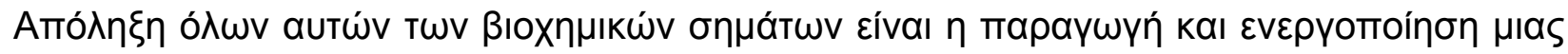

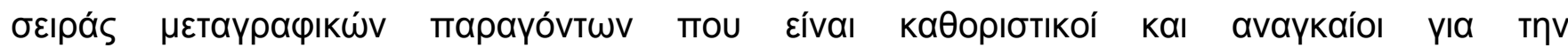

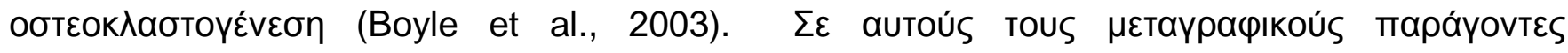

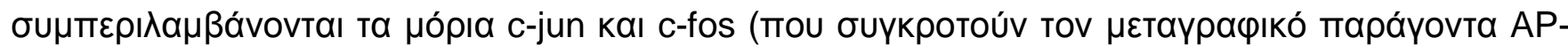

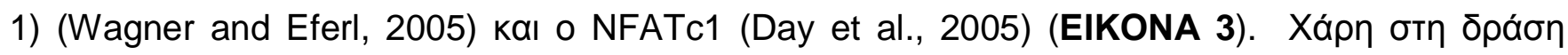

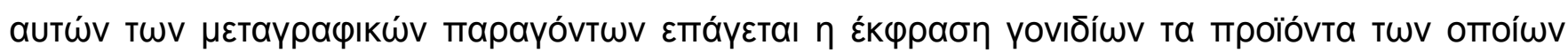

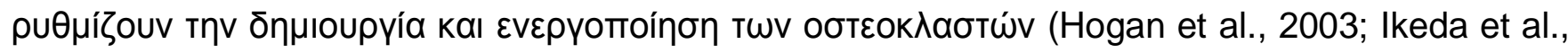
2004; Koga et al., 2005; Takayanagi, 2007b; Teitelbaum, 2004).

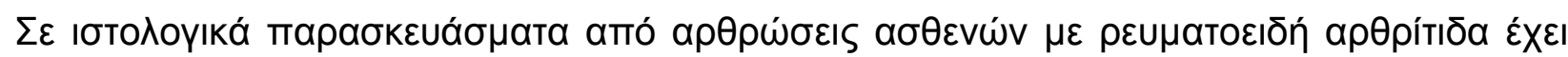

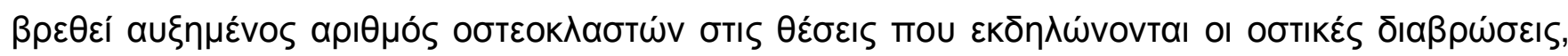

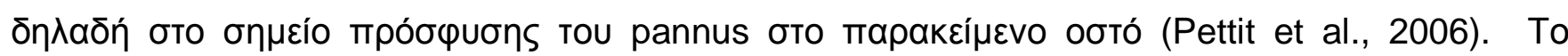

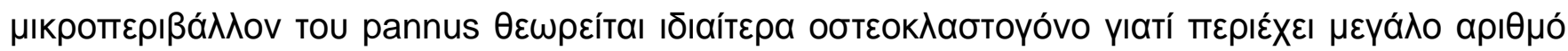

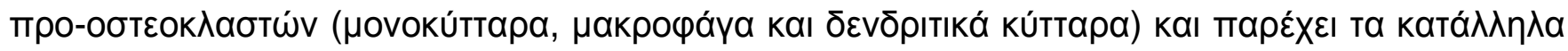

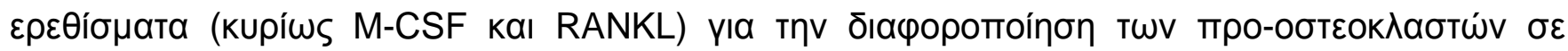

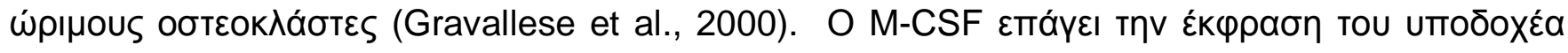

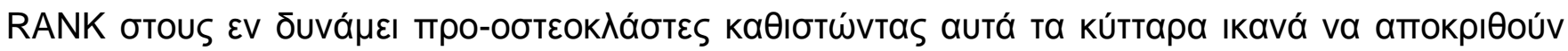




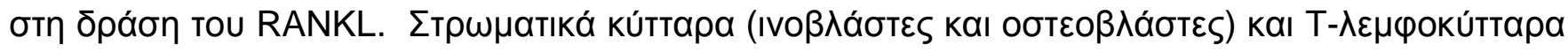

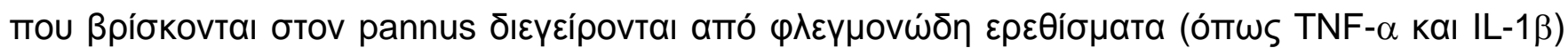

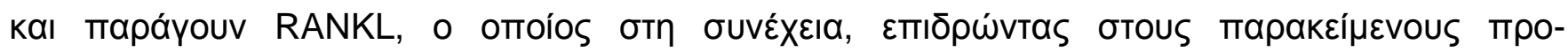

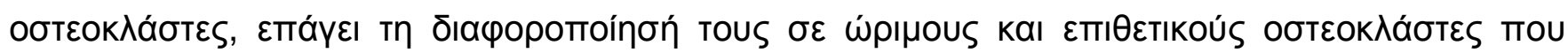

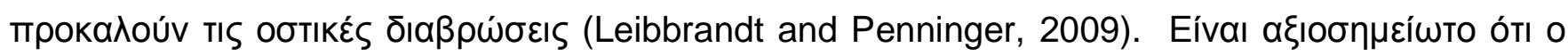
TNF- $\alpha$ (Kanazawa and Kudo, 2005; Kobayashi et al., 2000; Romas et al., 2002) kaı $\eta$ IL-17

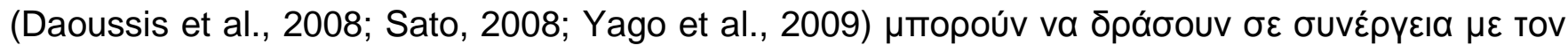

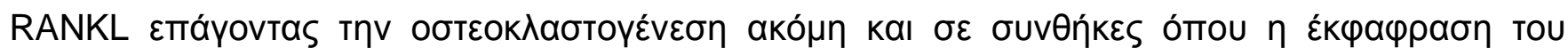

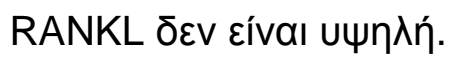




\subsection{INTEPAEYKINH 27 (IL-27)}

\subsection{1 $\Delta$ ouń tns IL-27}

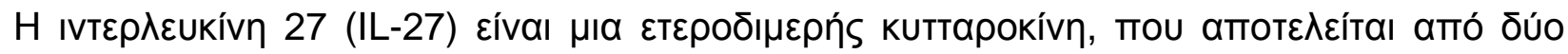

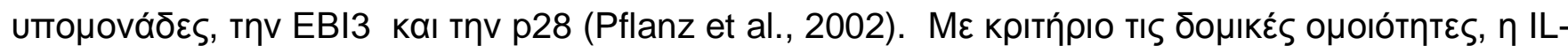

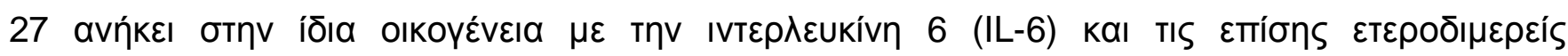

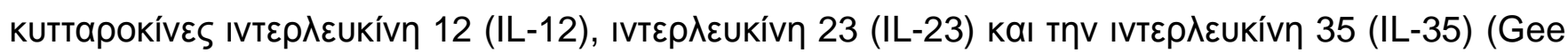

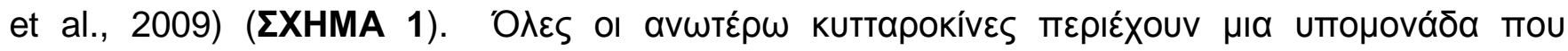

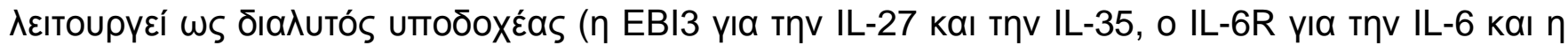

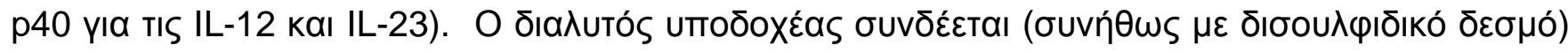

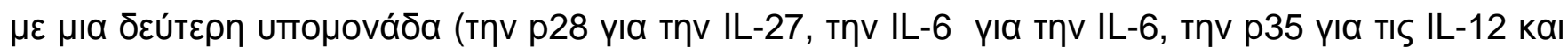

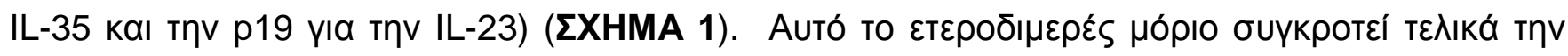

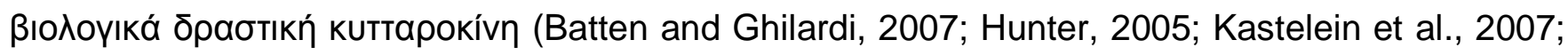

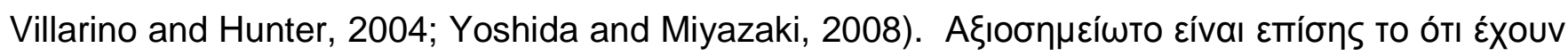

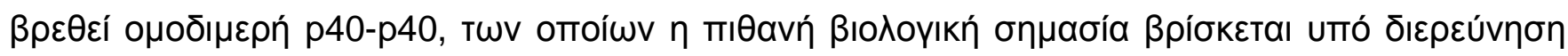

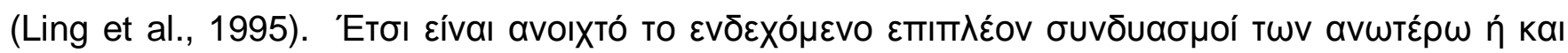

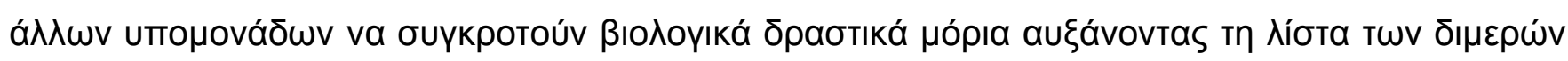
KUTTapokivúv.

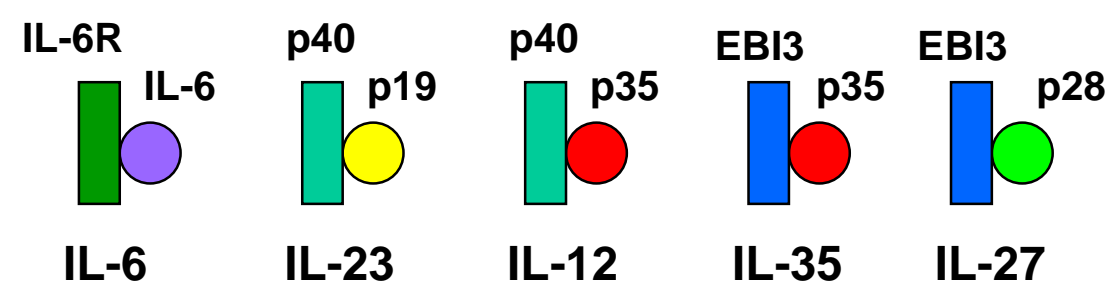

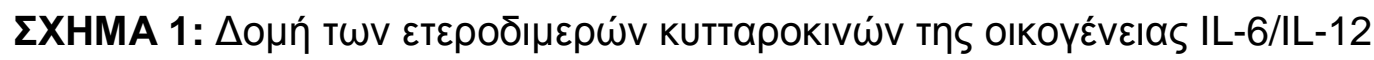




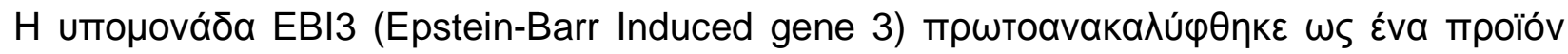

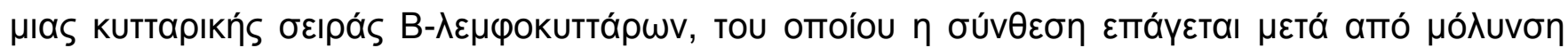

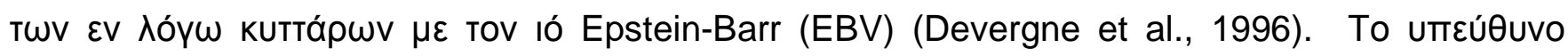

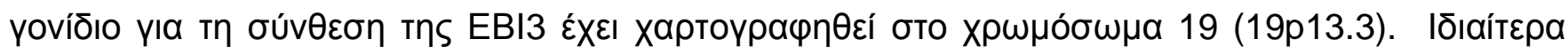

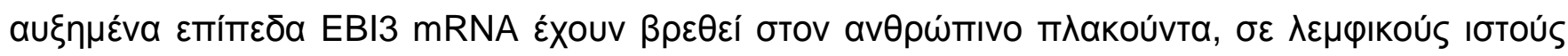

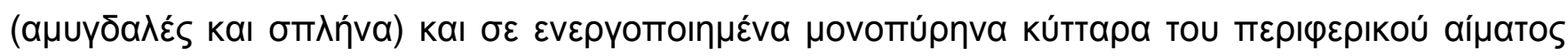

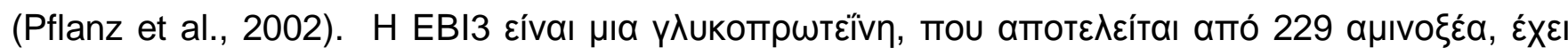

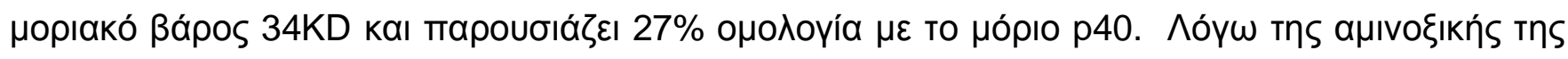

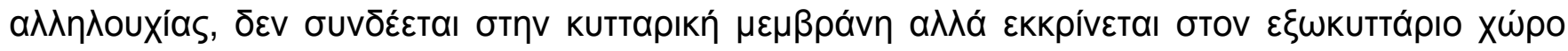

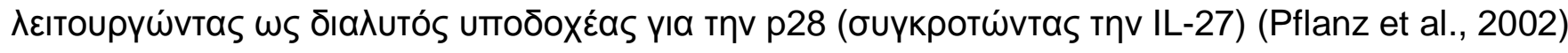

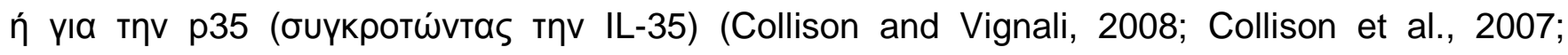
Niedbala et al., 2007).

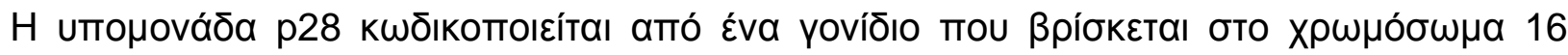

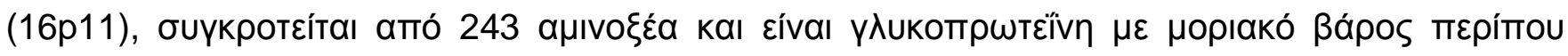

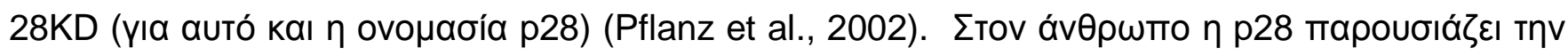

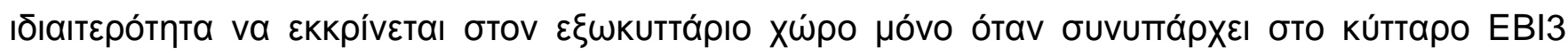

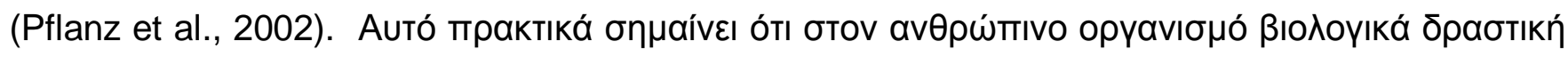

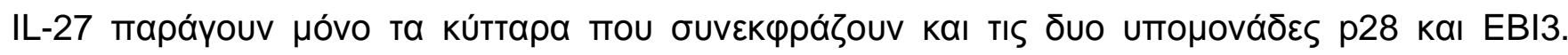

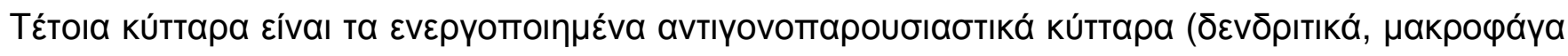

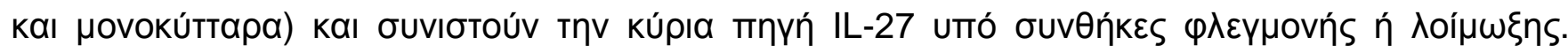

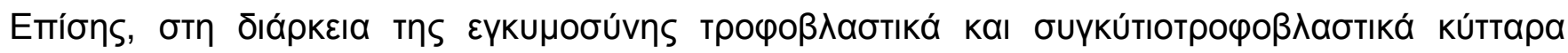
тарáyouv IL-27 (Coulomb-L'Hermine et al., 2007). 


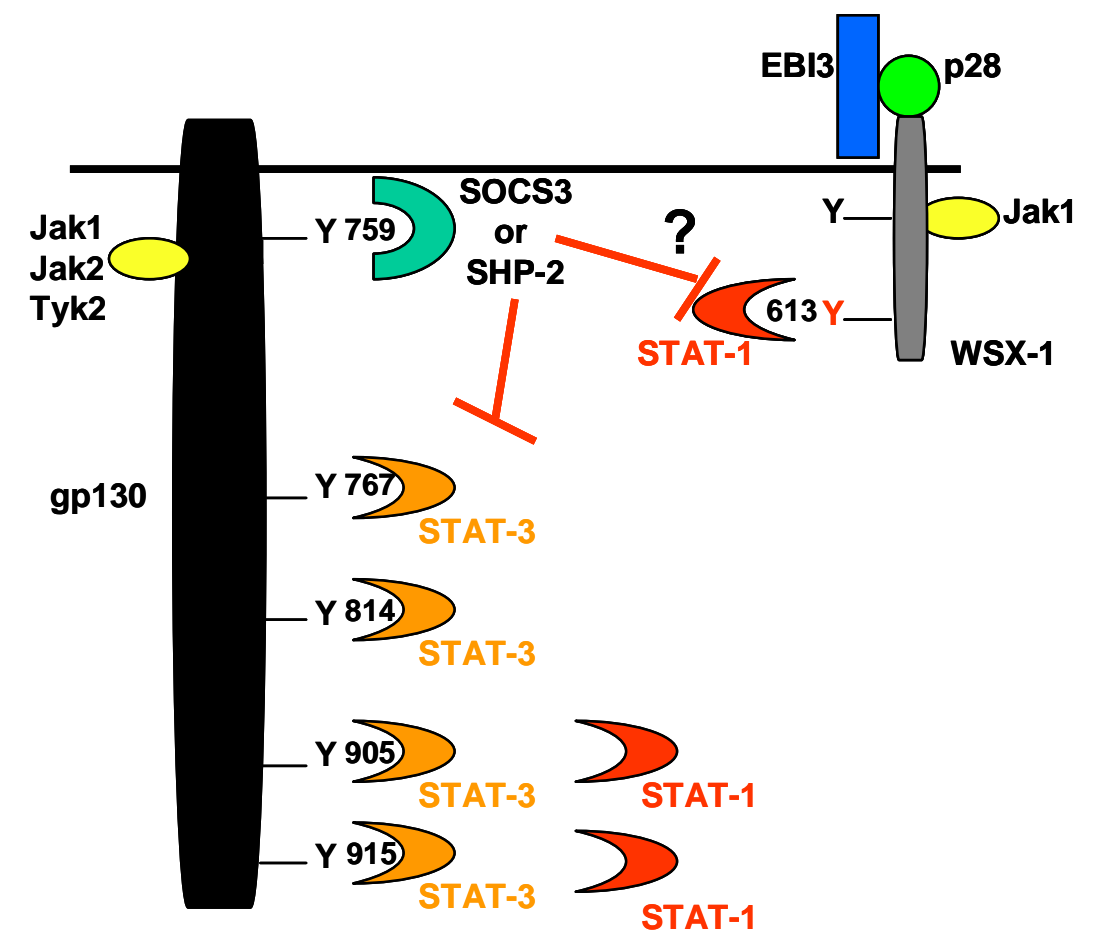

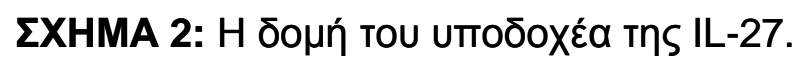

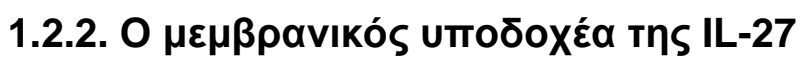

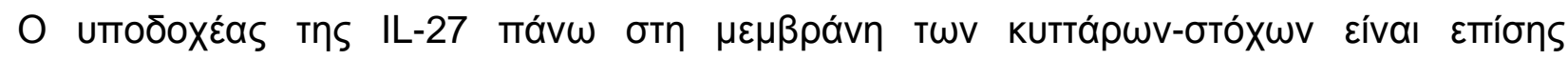

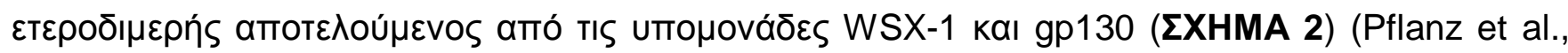

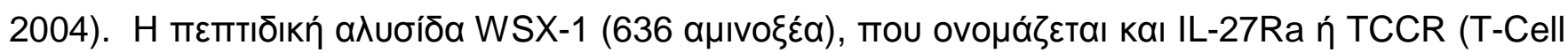

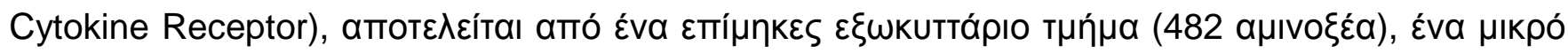

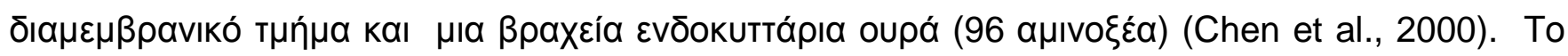

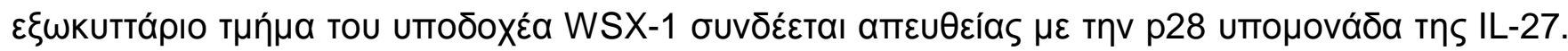

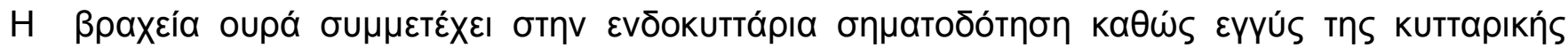

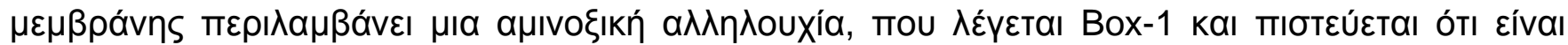

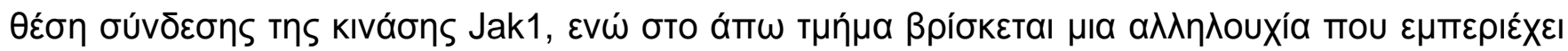

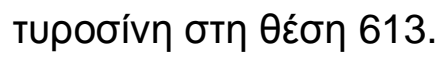




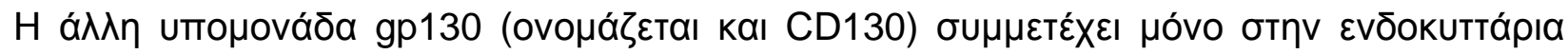

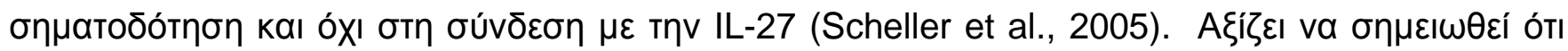

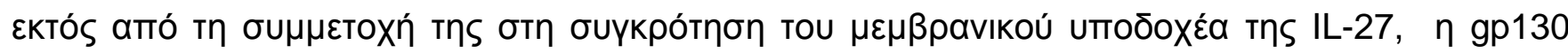

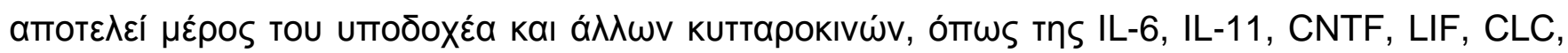
OSM kaı CT-1 (Boulanger and Garcia, 2004; Bravo and Heath, 2000; Murakami et al., 2004;

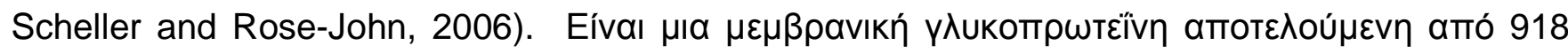
a

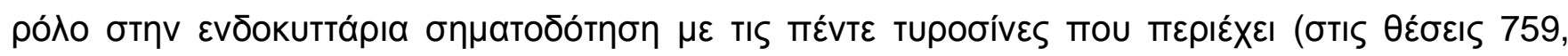
767, 814, 905 kaı 915) (IXHMA 2) (Ernst and Jenkins, 2004; Kamimura et al., 2003; Schmitz et

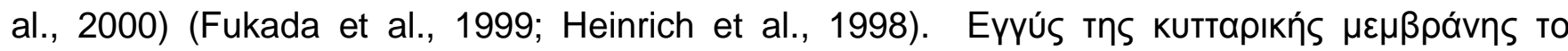

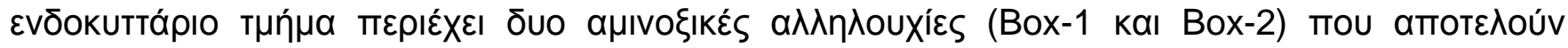

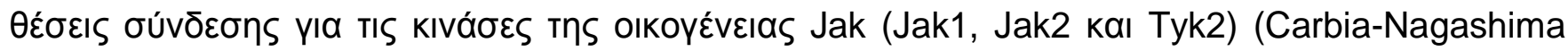
and Arzt, 2004; Chow et al., 2002; Kurth et al., 2000). 


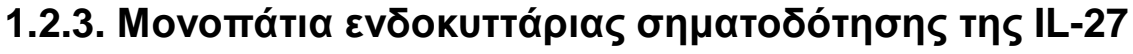

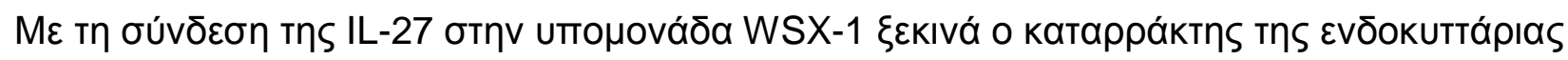

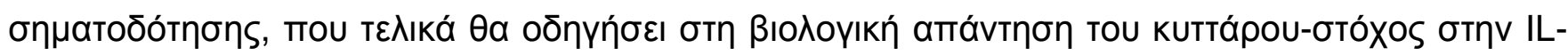

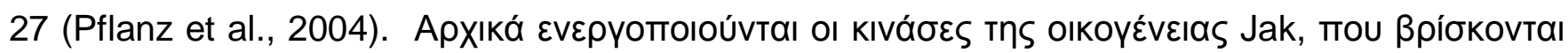

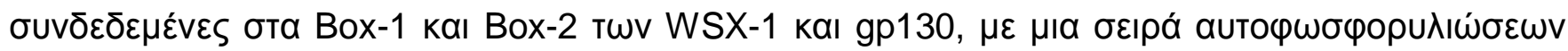

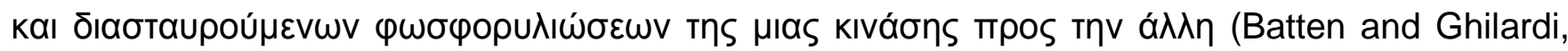

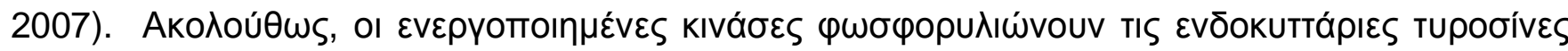

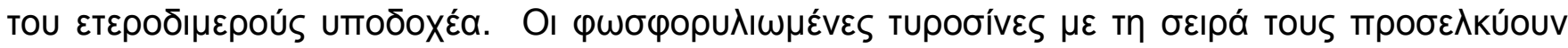

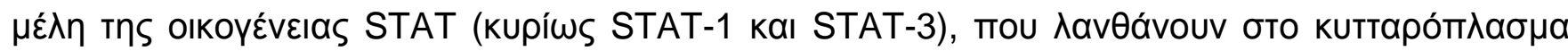

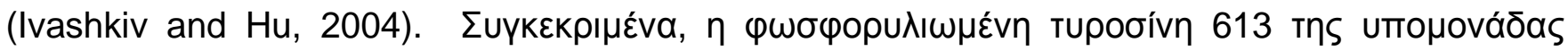

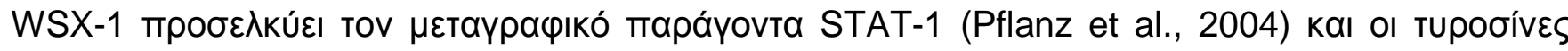

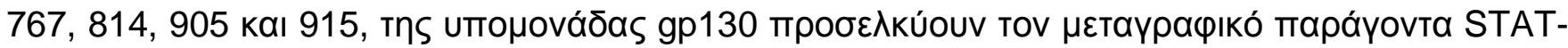

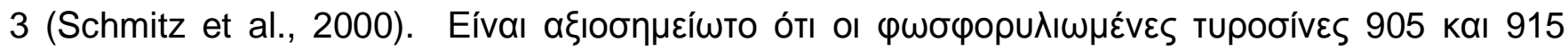

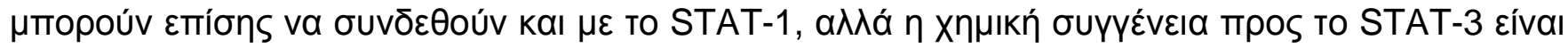

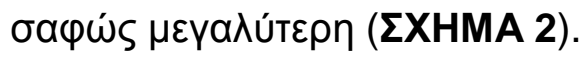

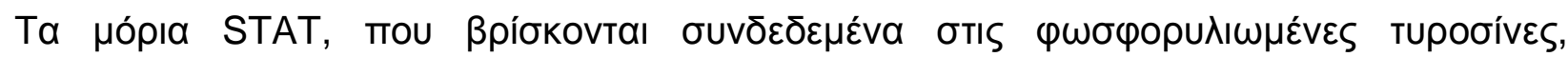

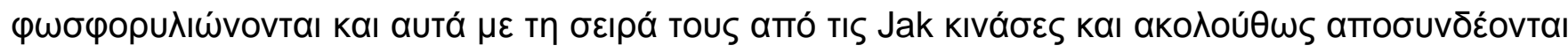

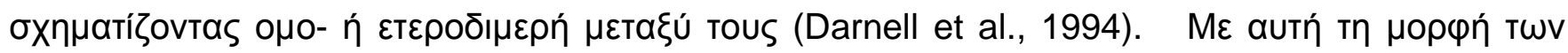

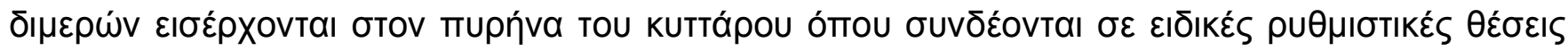

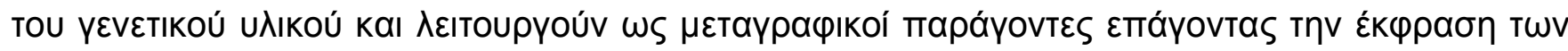

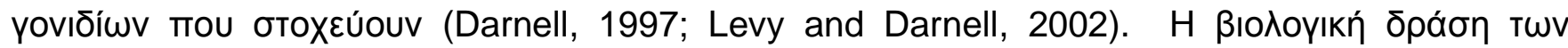

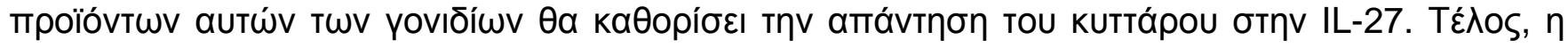

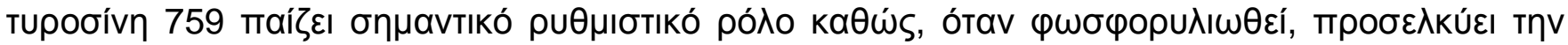




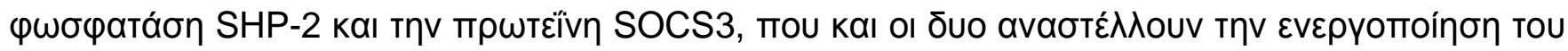

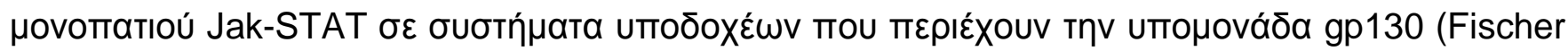
et al., 2004; Sengupta et al., 1996). 


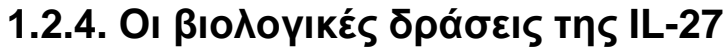

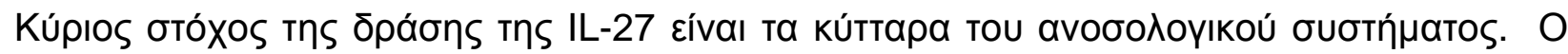

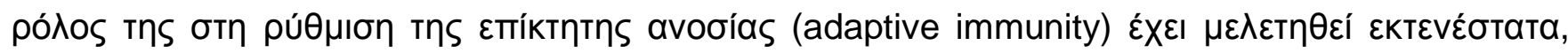

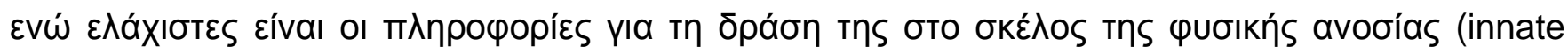

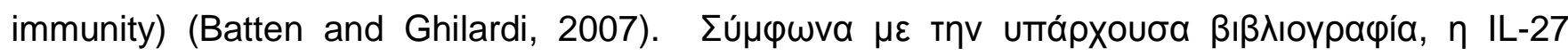

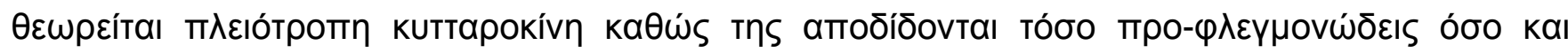

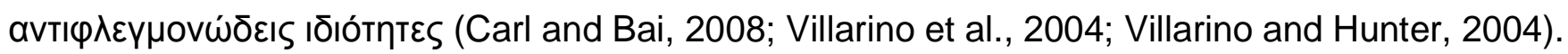

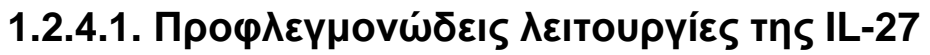

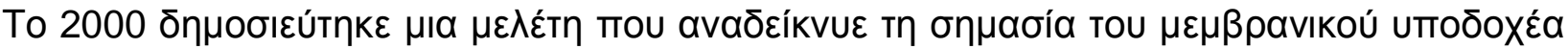

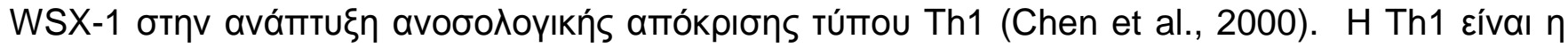

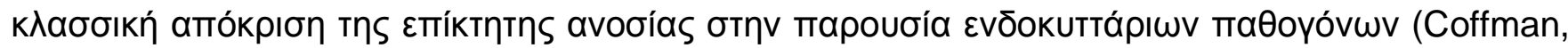

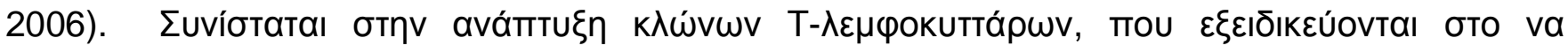

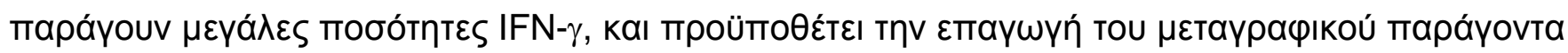

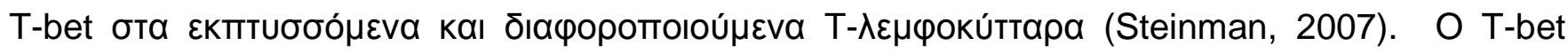

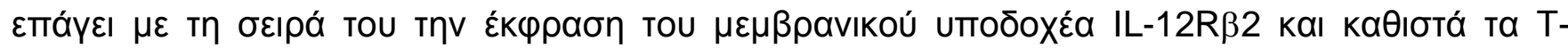

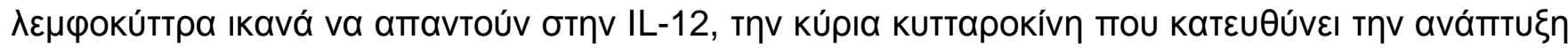

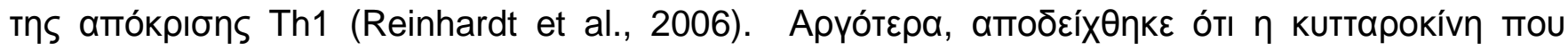

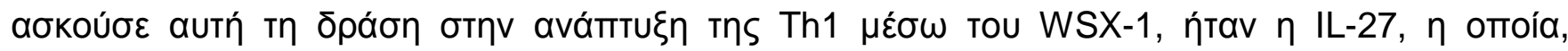

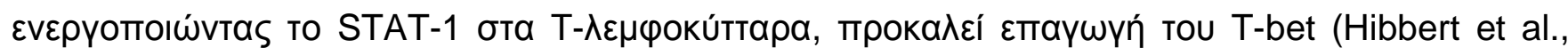
2003; Kamiya et al., 2004; Lucas et al., 2003; Owaki et al., 2006; Takeda et al., 2003).

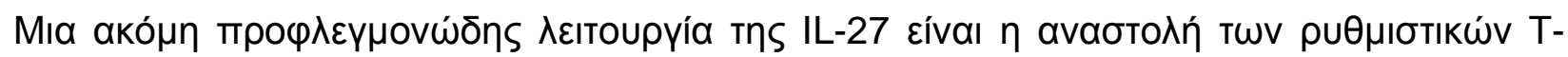

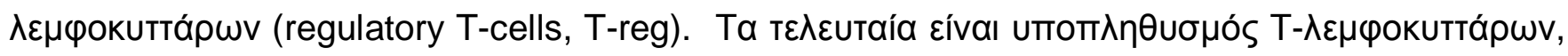




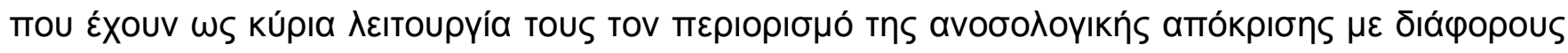

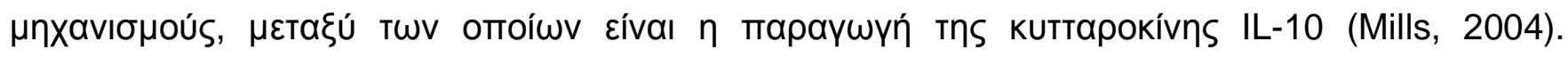

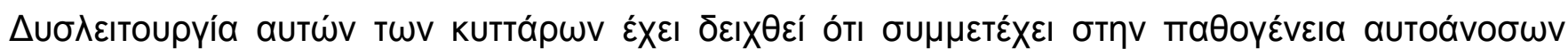

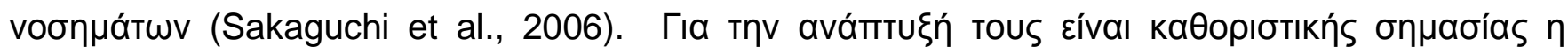

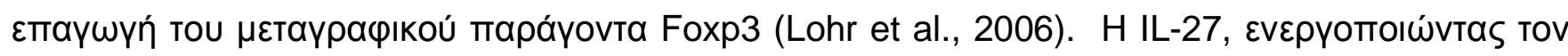

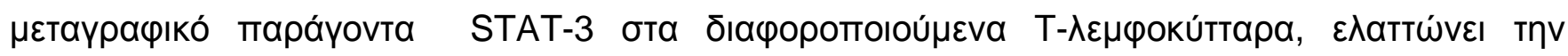

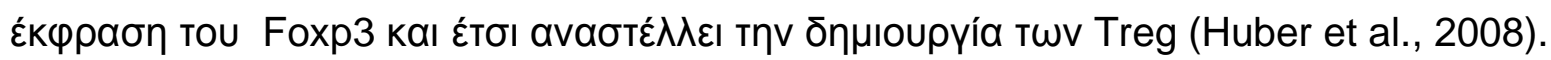




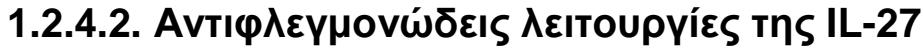

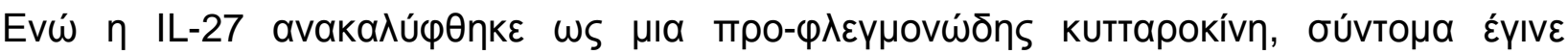

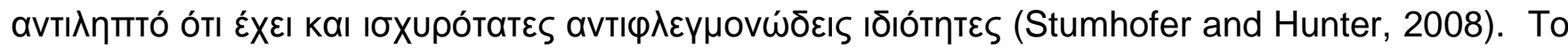

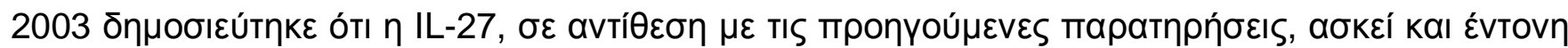

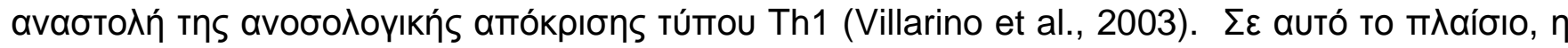

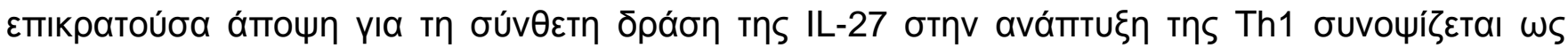

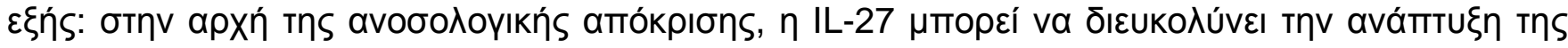

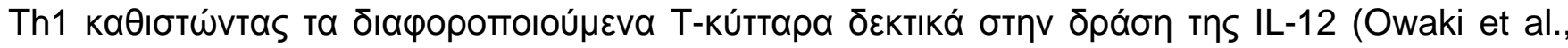

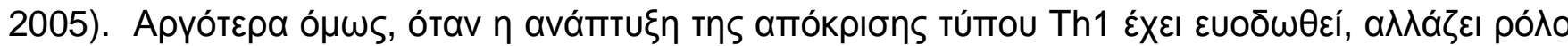

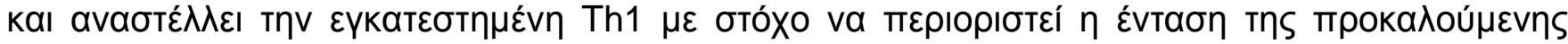

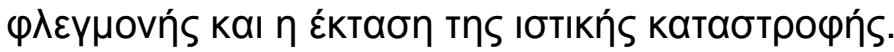

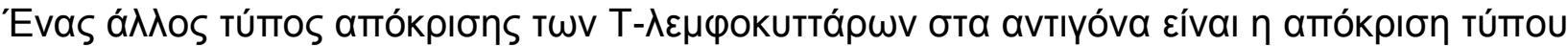

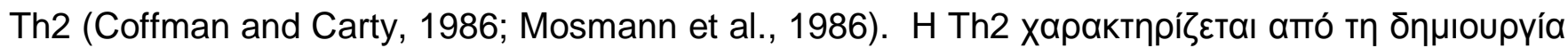

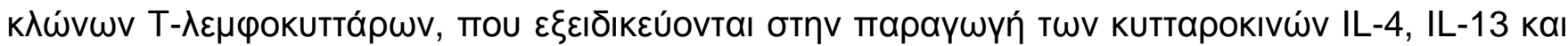

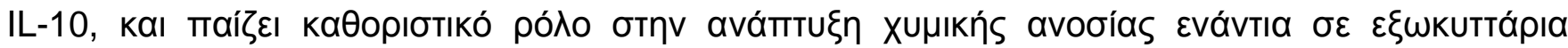

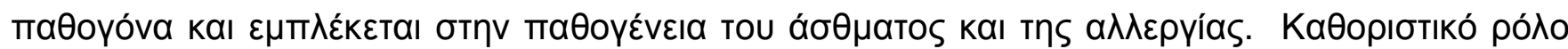

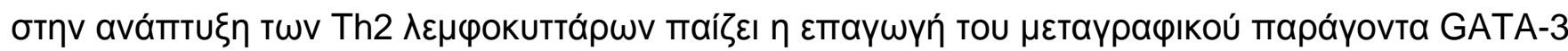

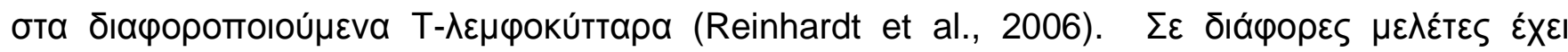

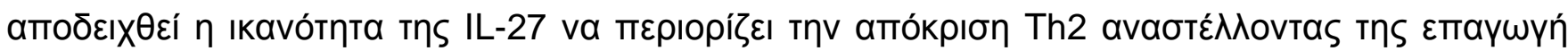
Tou GATA-3 (Lucas et al., 2003).

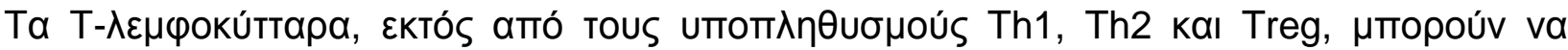

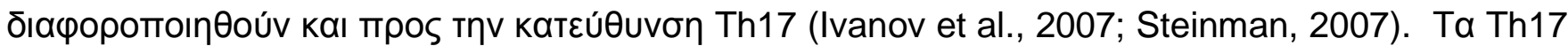

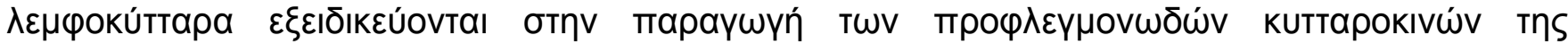


оıкоү́vદıаS IL-17 (Gaffen, 2009; Kolls and Linden, 2004; Miossec et al., 2009; Weaver et al.,

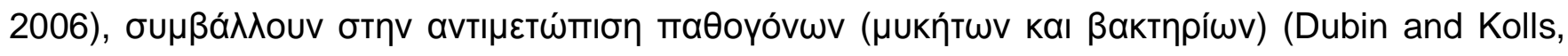

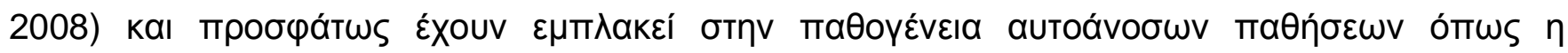

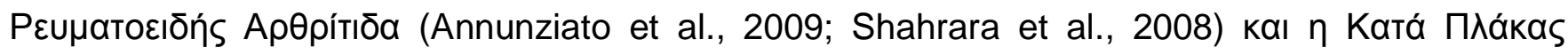

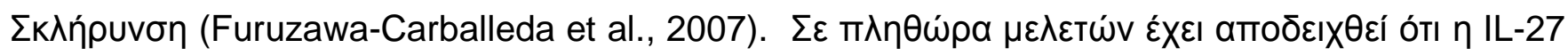

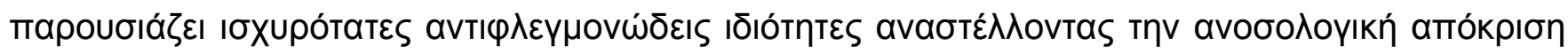
тúтоu Th17 (Batten et al., 2006; Colgan and Rothman, 2006; Diveu et al., 2009; Fitzgerald et al.,

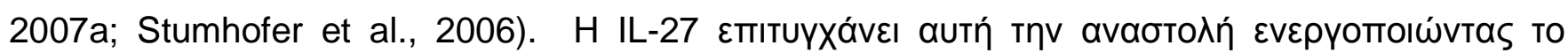

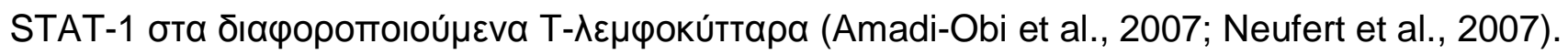

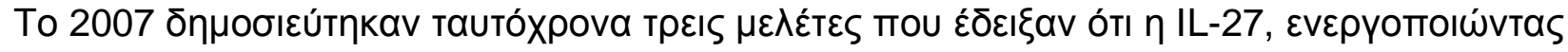

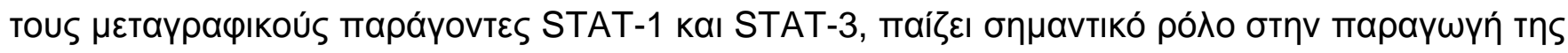

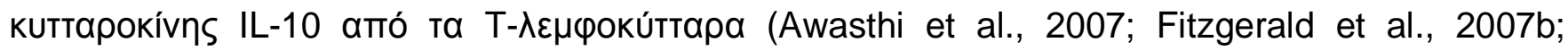

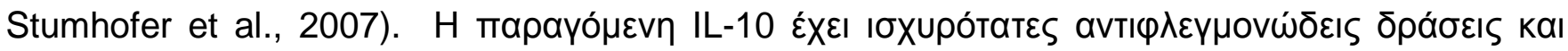

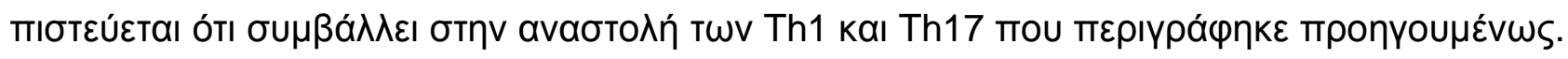




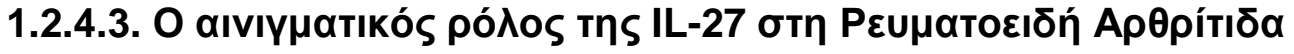

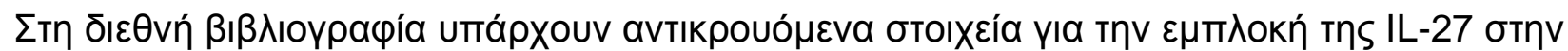

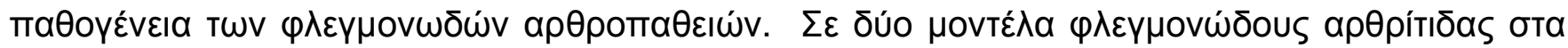

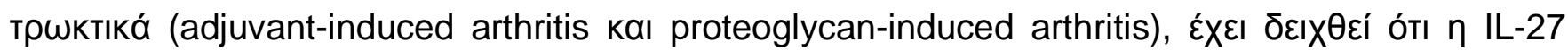

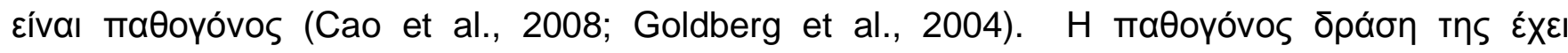

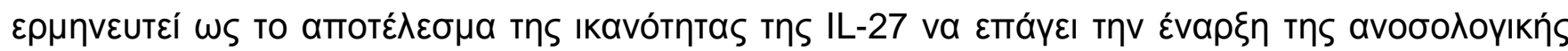

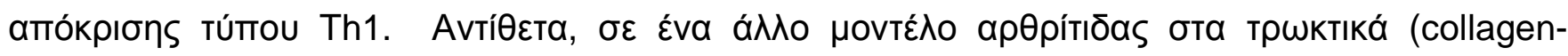

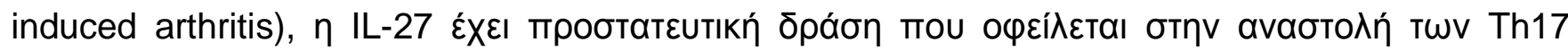
(Niedbala et al., 2008).

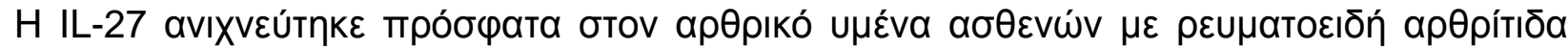

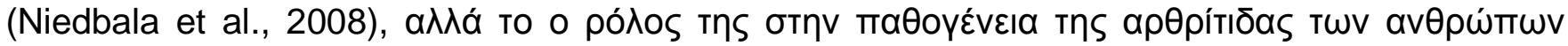

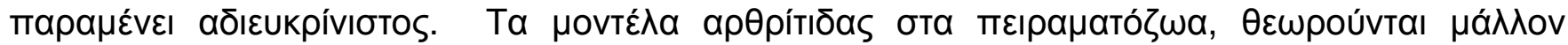

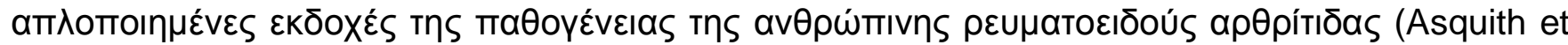

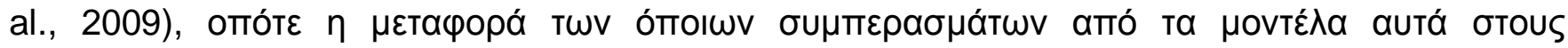

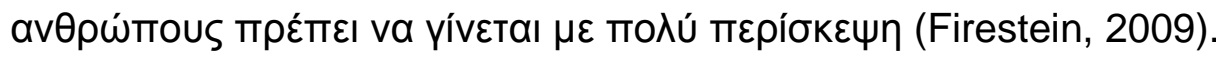

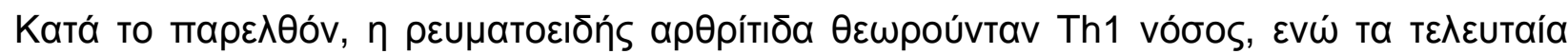

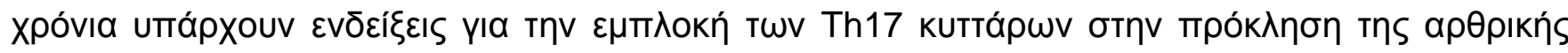

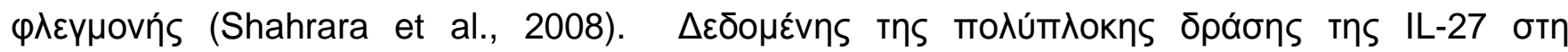

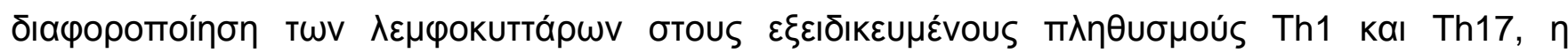

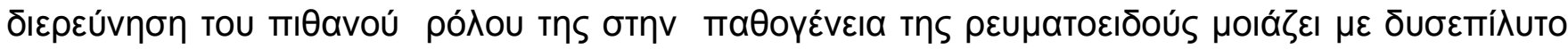
aíviүua.

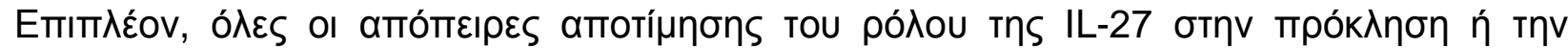

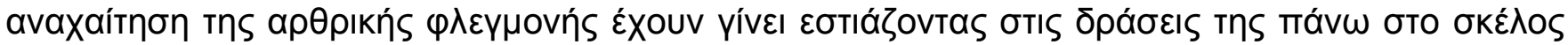




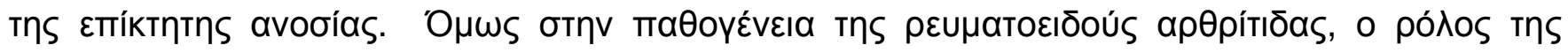

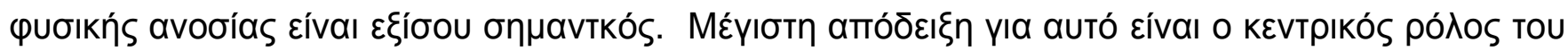

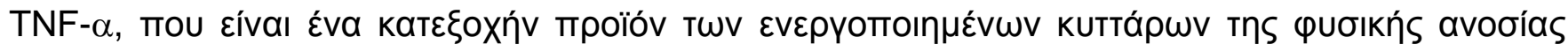

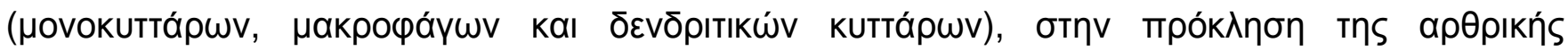
$\varphi \lambda \varepsilon \gamma \mu$ ovńs. 


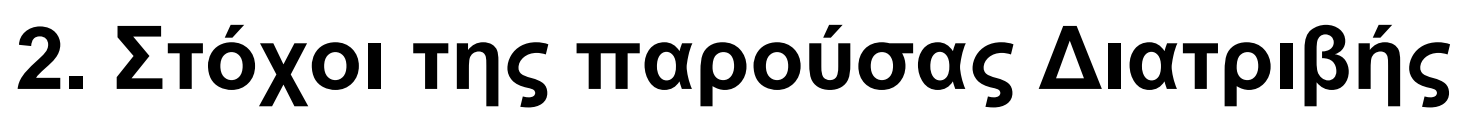




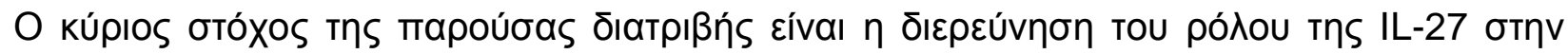

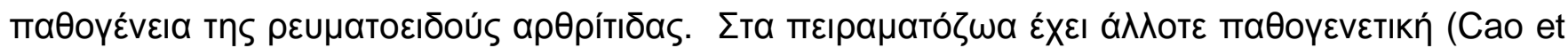

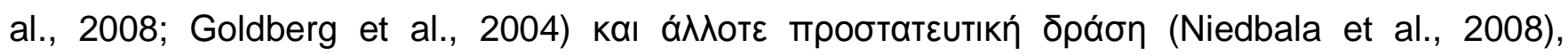

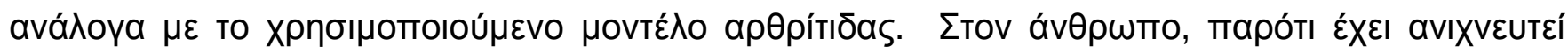

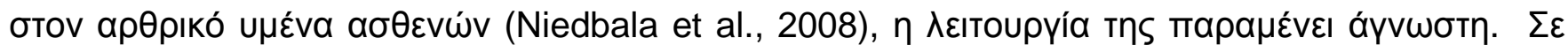

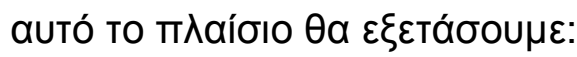

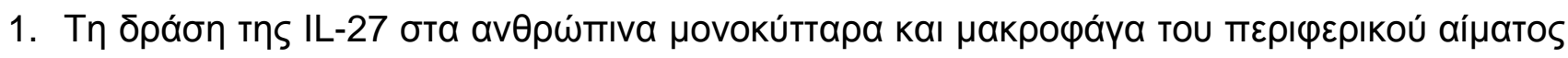

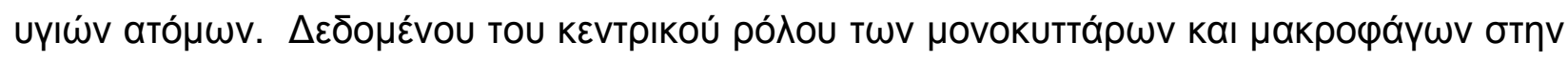

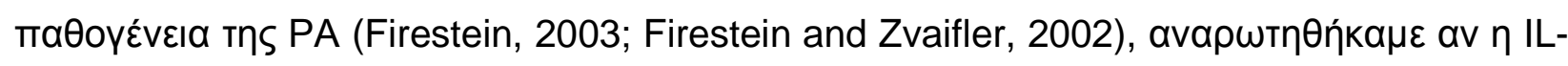

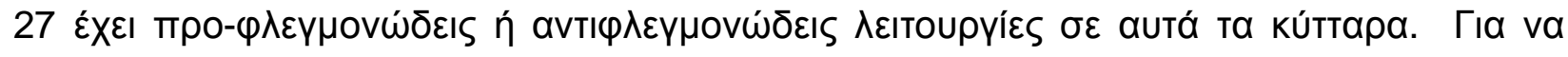

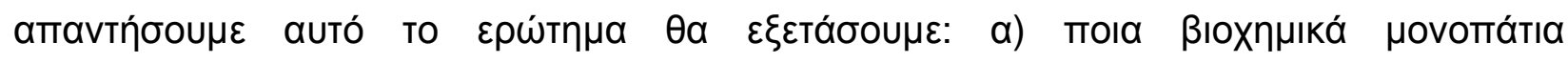

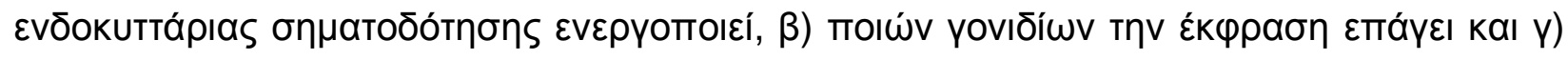

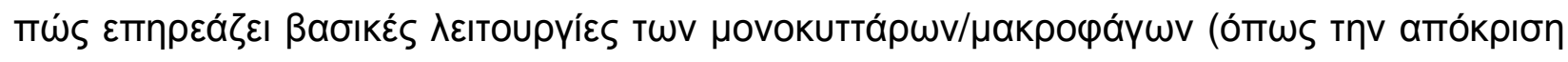

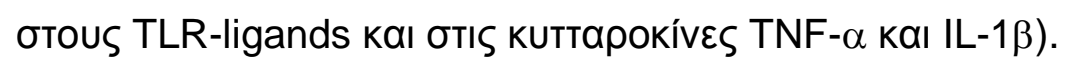

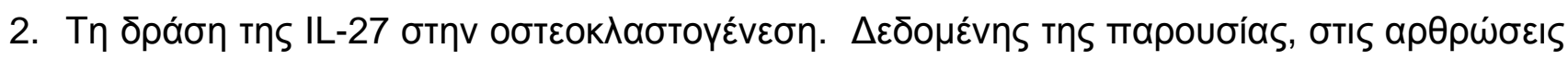

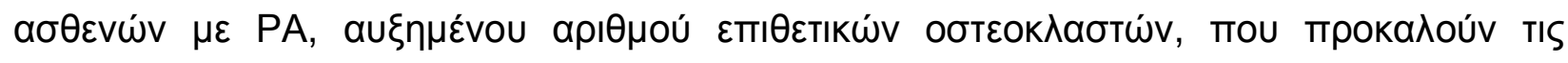

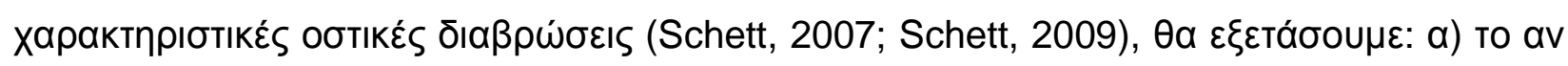

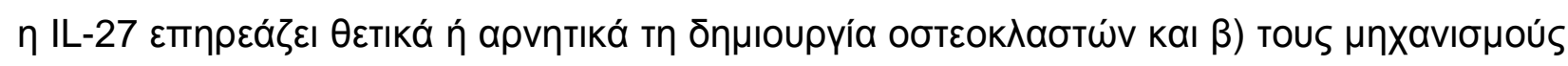
$\mu \varepsilon$ тоu оттоі́о

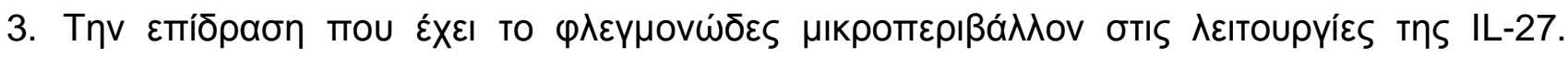

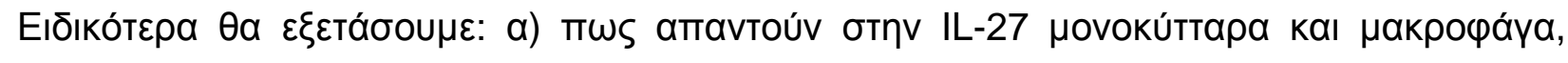

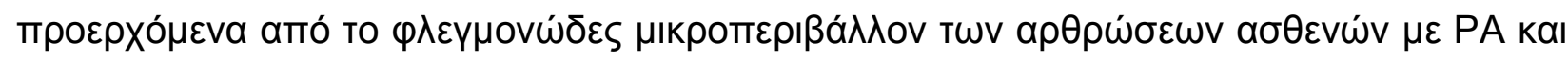

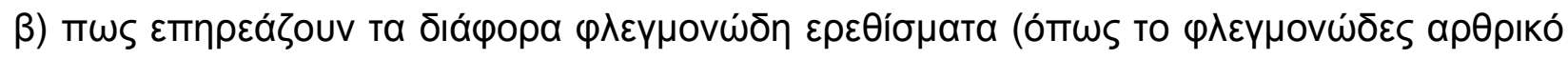




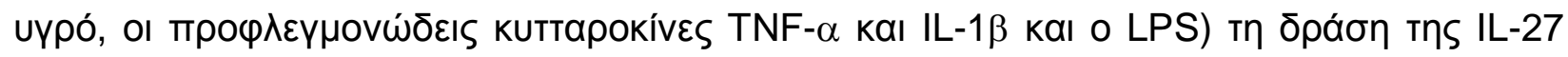

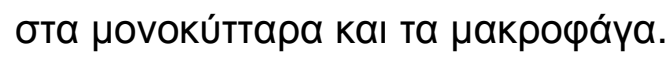




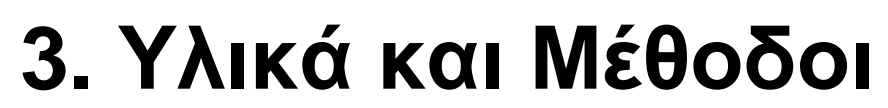




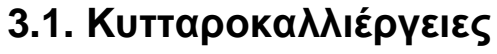

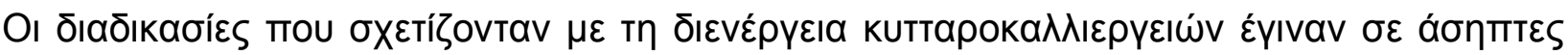

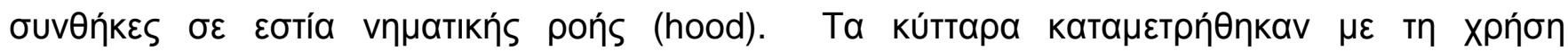

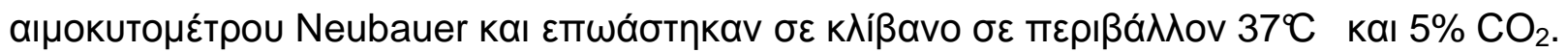

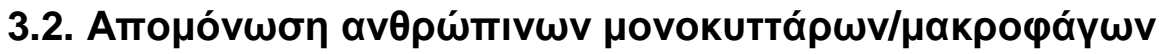

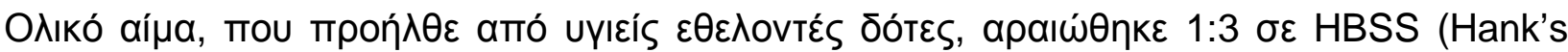

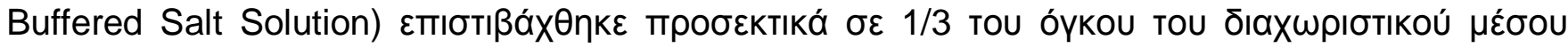

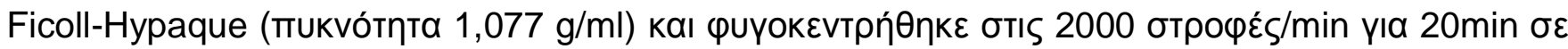

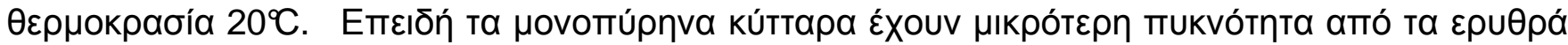

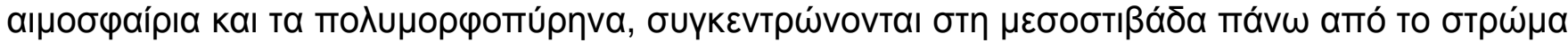

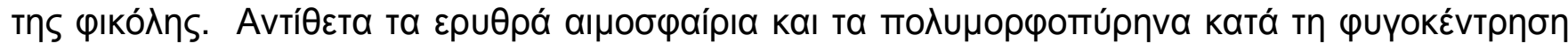

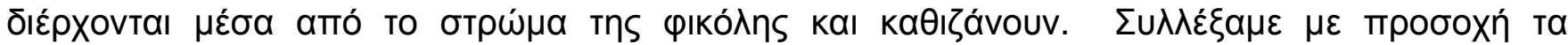

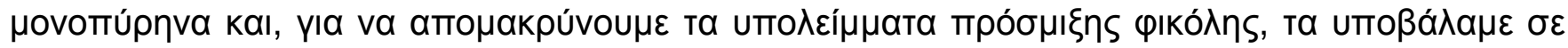

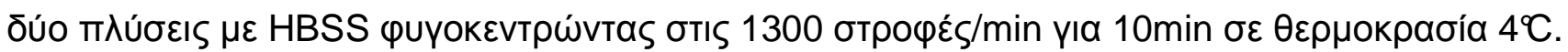

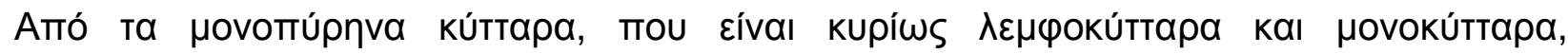

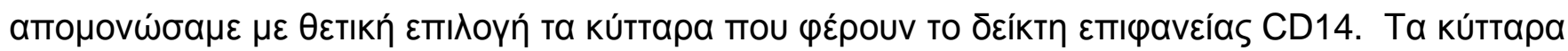

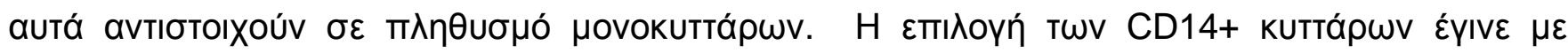

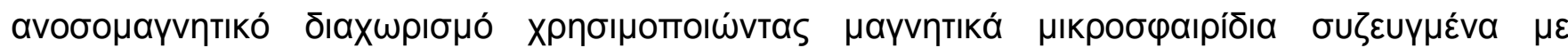

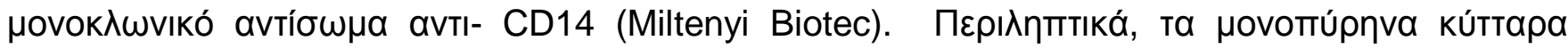

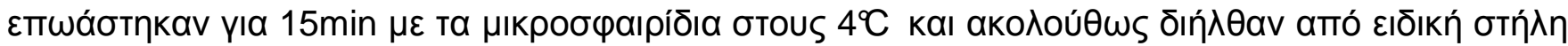

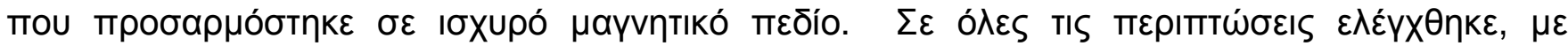

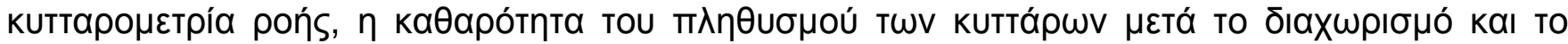




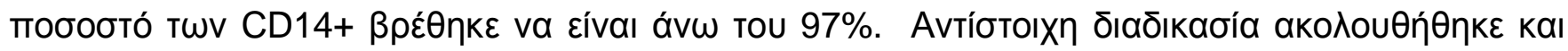

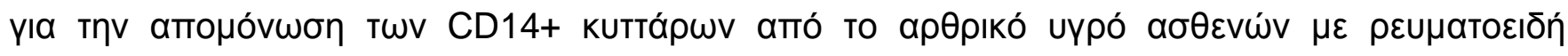
арӨрі́тıठа.

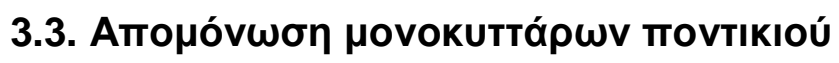

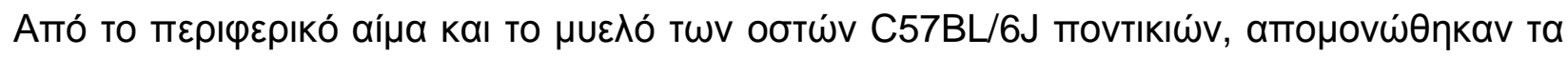

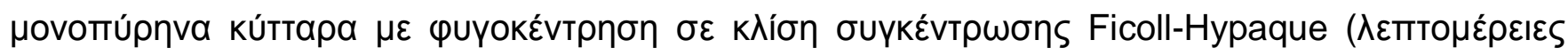

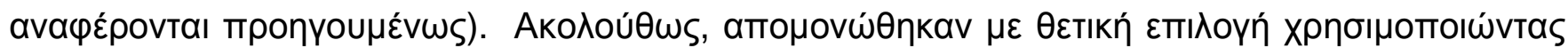

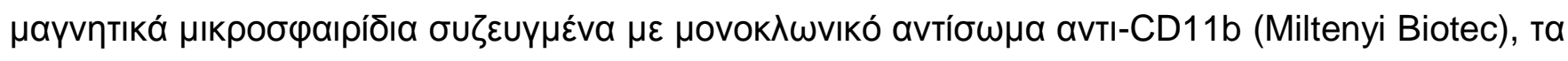

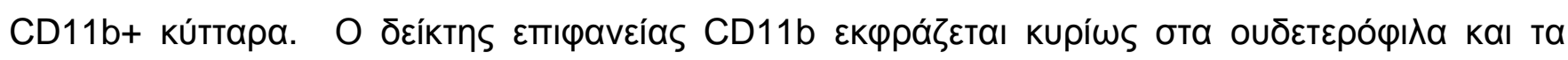

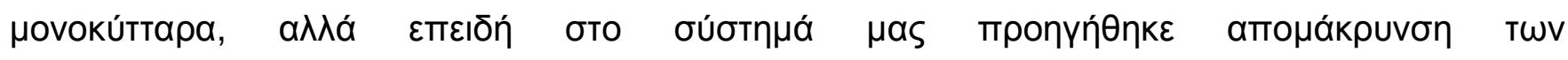

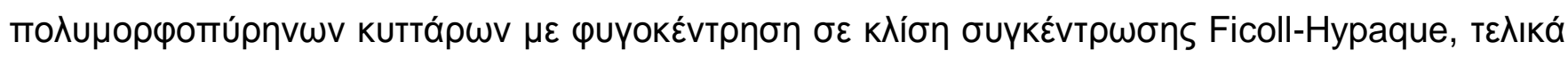

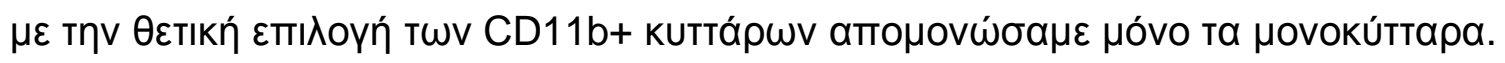

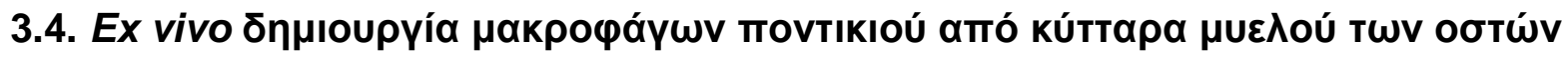

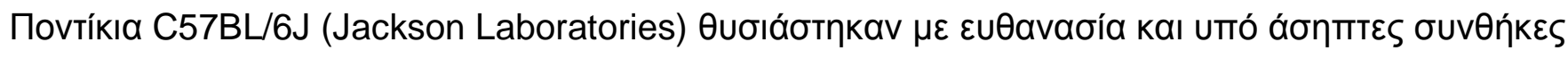

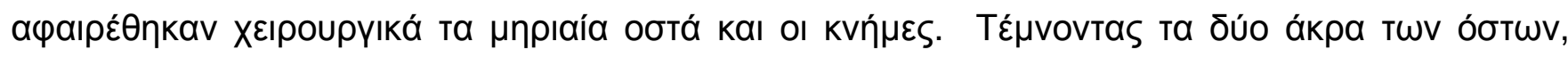

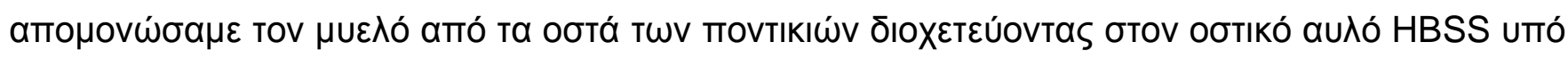

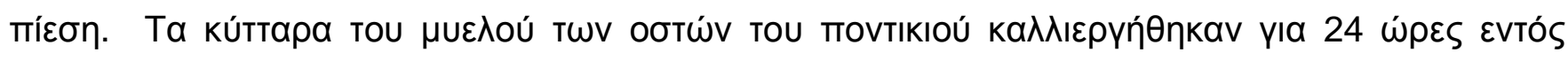

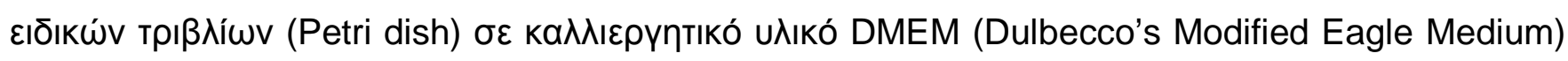

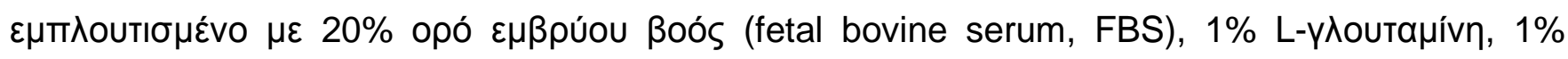

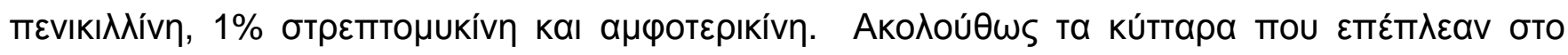

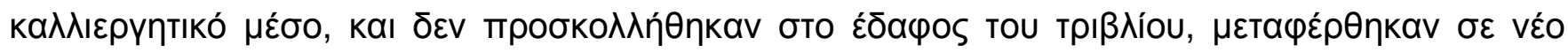




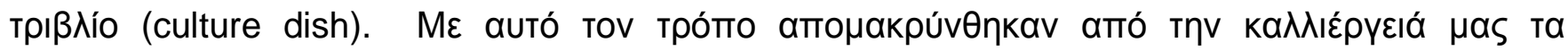

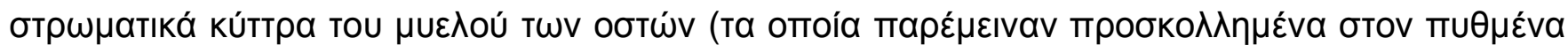

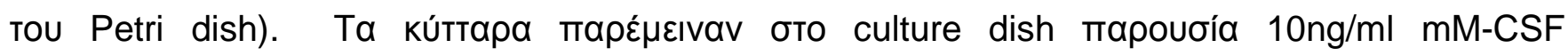

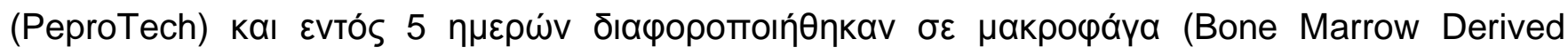
Macrophages, BMDM).

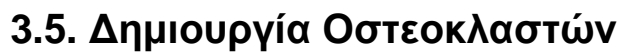

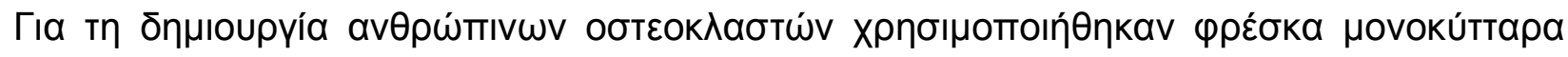

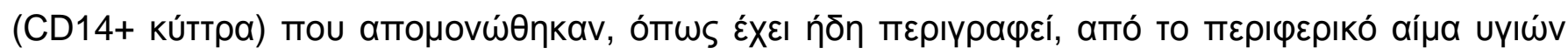

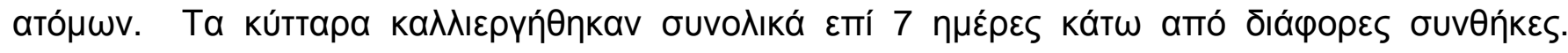

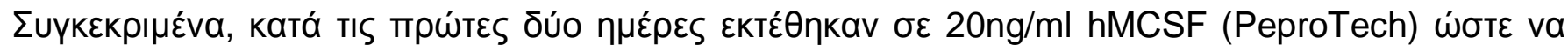

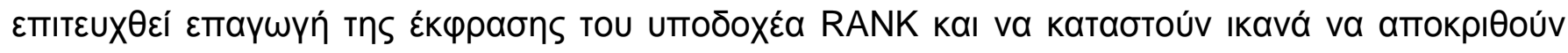

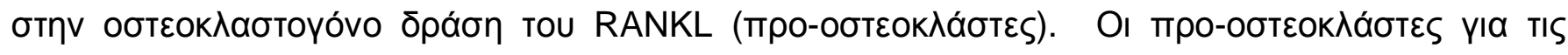

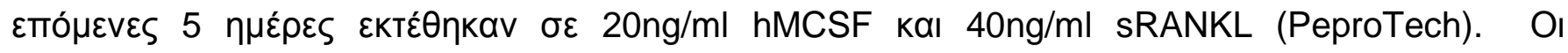

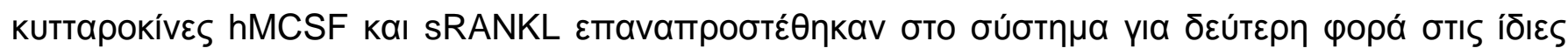

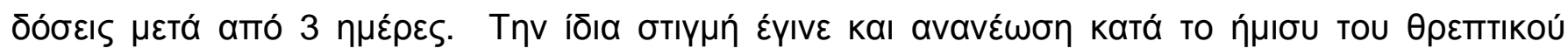

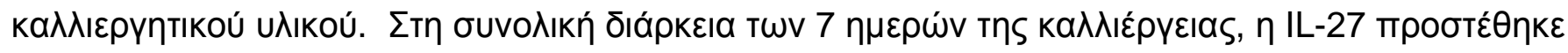

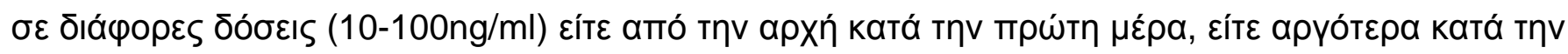

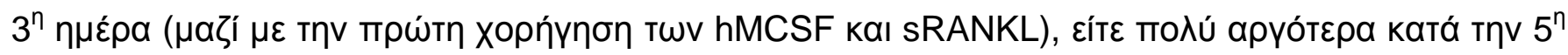

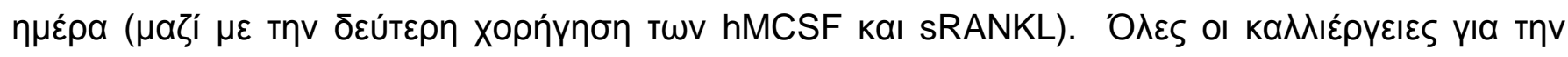

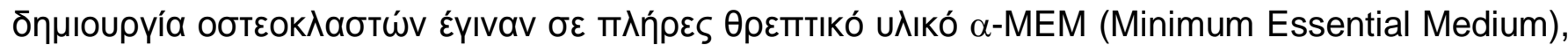

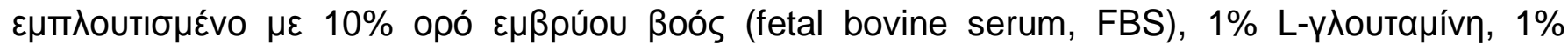

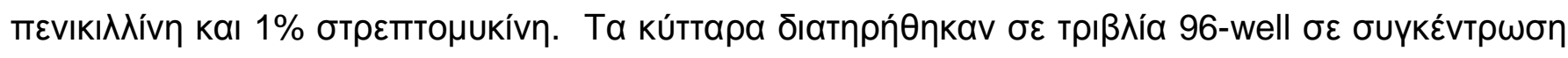




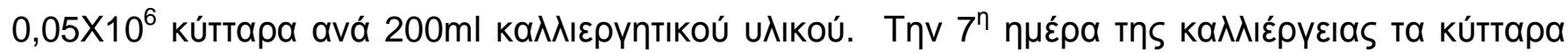

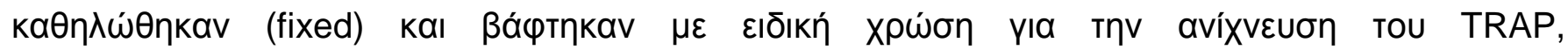
хрпбıнотоіи́vтаs то Acid Phosphatase Leukocyte diagnostic kit (Sigma, San Diego, CA),

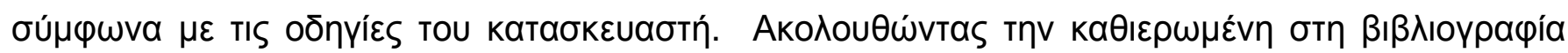

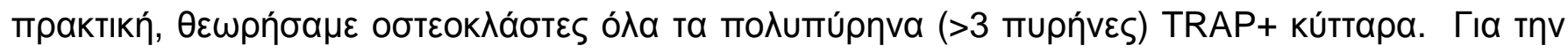

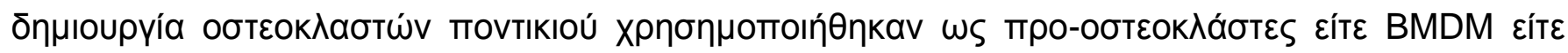

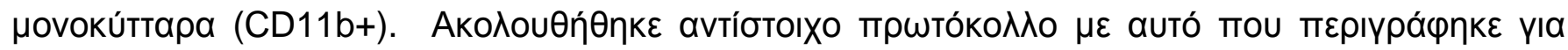

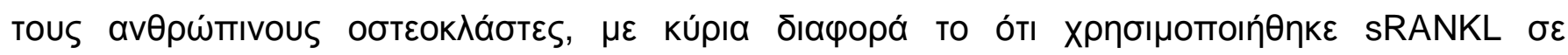

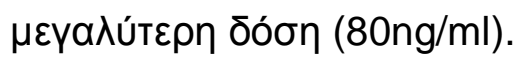

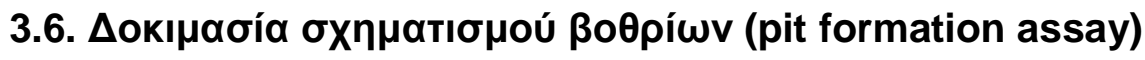

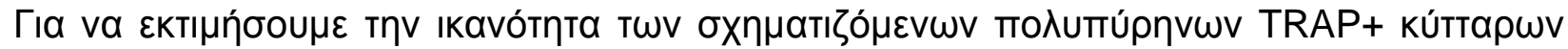

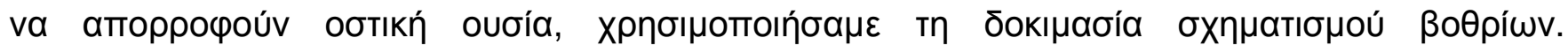

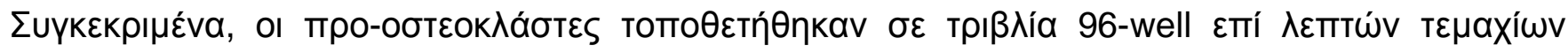

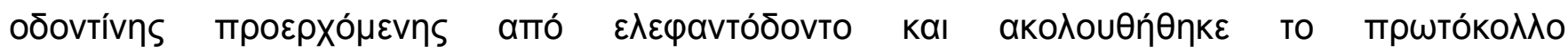

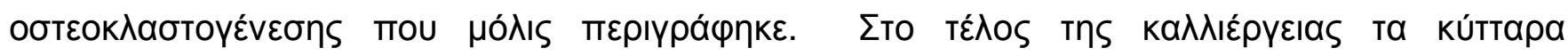

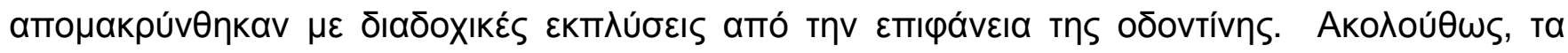

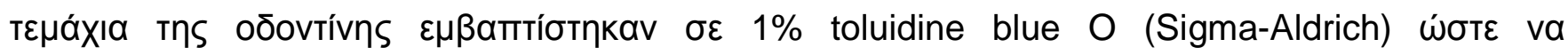

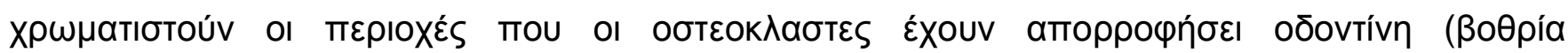

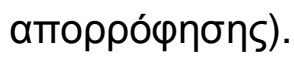




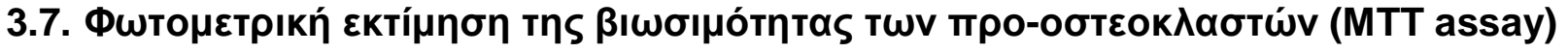

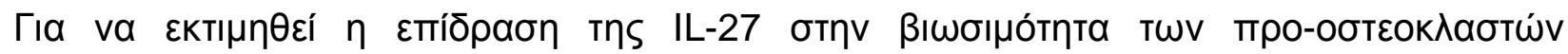

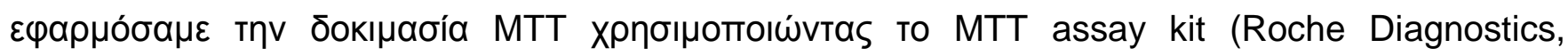

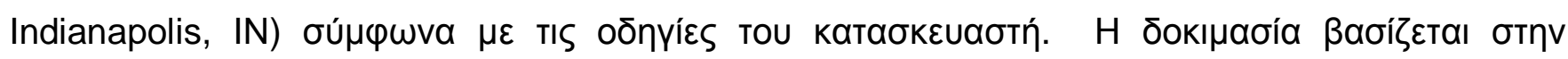

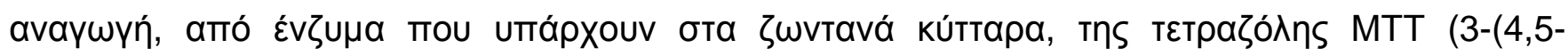

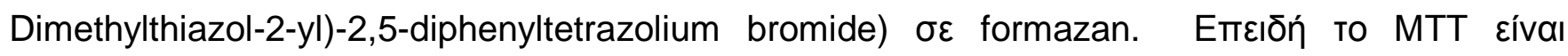

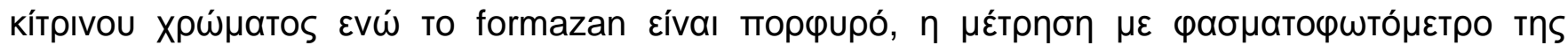

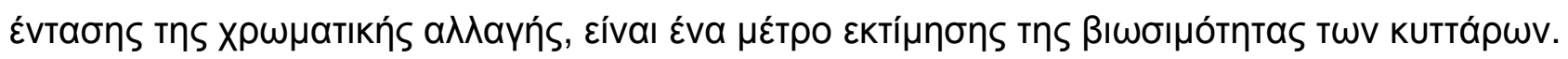

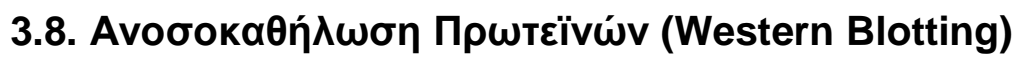

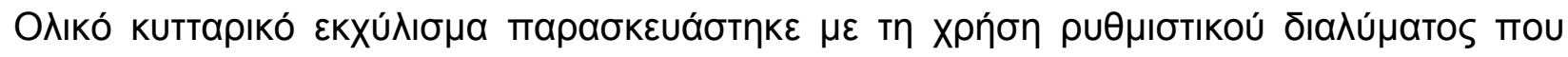

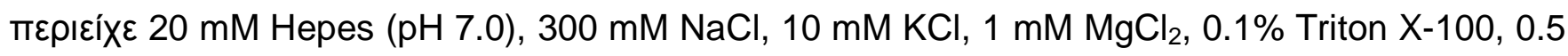
mM DTT, 20\% glycerol kaı $1 \times$ proteinase inhibitor cocktail (Roche, Basel, Switzerland).

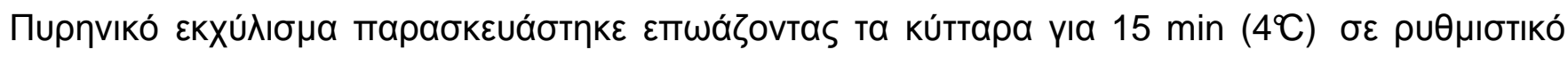

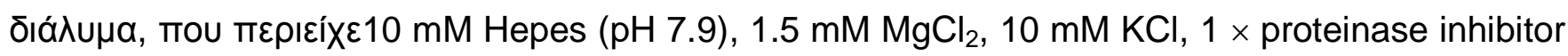

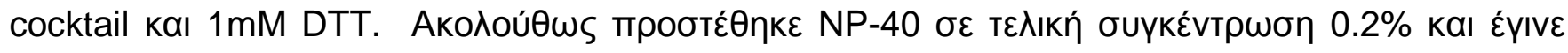

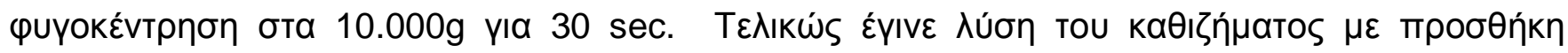

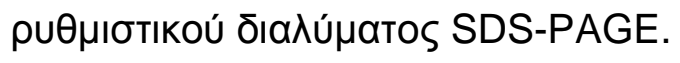

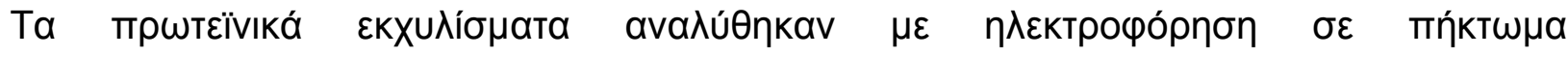

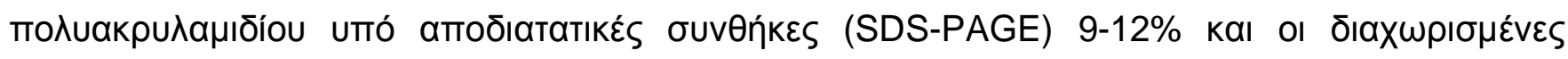

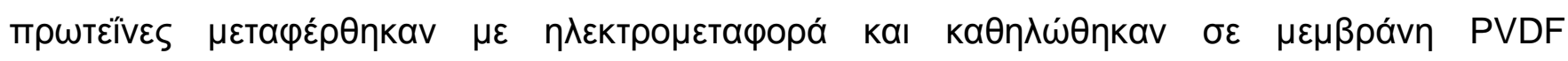

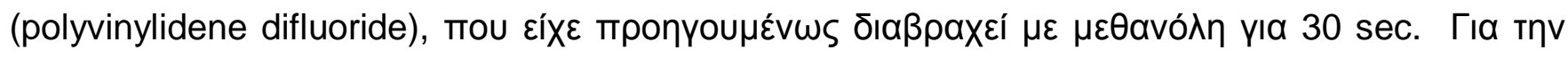

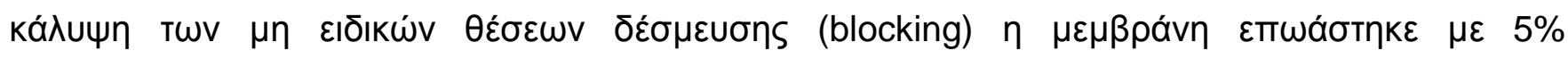




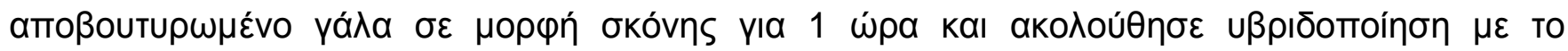

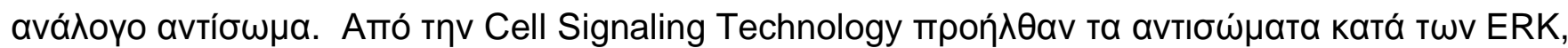

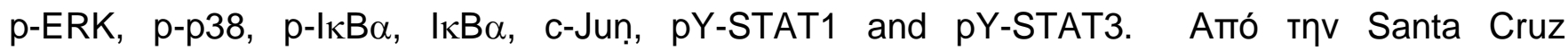

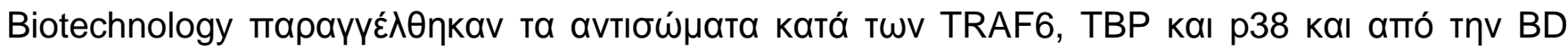

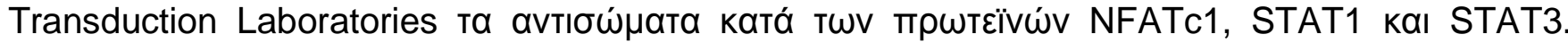

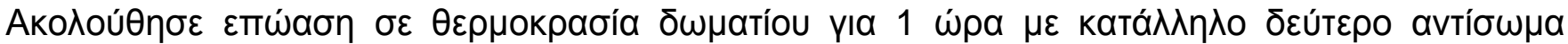

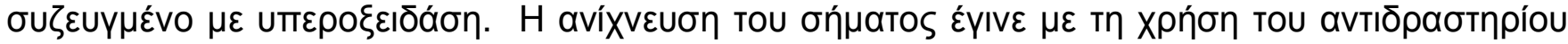

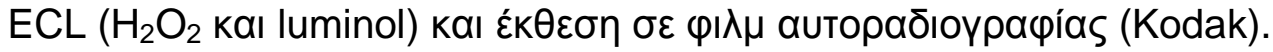

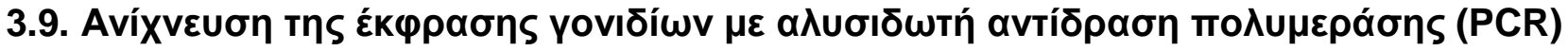

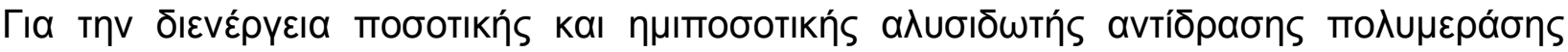

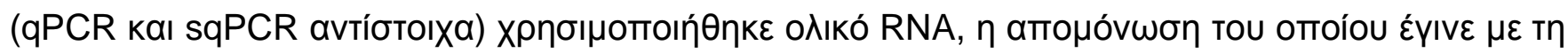

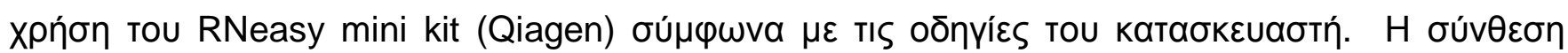

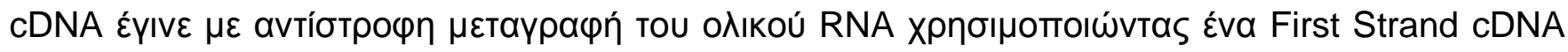

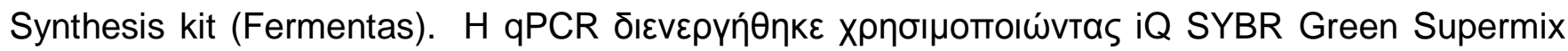

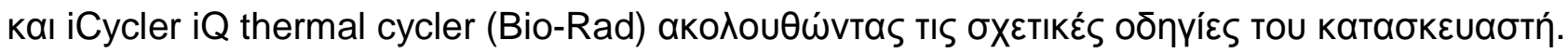

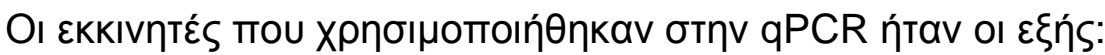

- hGAPDH forward 5'-ATCAAGAAGGTGGTGAAGCA-3'

hGAPDH reverse 5'-GTCGCTGTTGAAGTCAGAGGA-3'

- hSOCS1 forward 5'-TGTTGTAGCAGCTTAACTGTATC-3'

hSOCS1 reverse 5'-AGAGGTAGGAGGTGCGAGT-3'

- hSOCS3 forward 5'-CACTCTTCAGCATCTCTGTCGGAAG-3'

hSOCS3 reverse 5'-CATAGGAGTCCAGGTGGCCGTTGAC-3' 
- hCXCL9 forward 5'-ATCAGCACCAACCAAGGGACT-3' hCXCL9 reverse 5'-GCTTTTTCTTTTGGCTGACCTG-3'

- hCXCL10 forward 5'-ATTTGCTGCCTTATCTTTCTG-3' hCXCL10 reverse 5'-TCTCACCCTTCTTTTTCATTGTAG-3'

- hSTAT-1 forward 5'-TGGGTTTGACAAGGTTCTT-3' hSTAT-1 reverse 5'-TATGCAGTGCCACGGAAAG-3'

- hIRF-1 forward 5'-GCACTAAGCGAAAATTGCA-3' hIRF-1 reverse 5'-GGGAGTTTCCTTCACATTCA-3'

- hCR1 forward 5'-GGATCCCTGCTGGCGGTTGTG-3' hCR1 reverse 5'-CCAGGGCGGCATTCATAGTTCAGA-3'

- hDEPP forward 5'-GGCCGCCCTGCTGTGTCC-3' hDEPP reverse 5'-CCTCTGGCTTGGCTGCTTTTCCTG-3'

- hCD163 forward 5'-CCAGTCCCAAACACTGTCCT-3' hCD163 reverse 5'-CACTCTCTATGCAGGCCACA-3'

- hPGDH forward 5'-GCTGGAGTGAATAATGAGAAAAAC-3' hPGDH reverse 5'-CAATAAACCGGCTGCTGTG-3'

- hTNF- $\alpha$ forward 5'-AATAGGCTGTTCCCATGTAGC-3' hTNF- $\alpha$ reverse 5'-AGAGGCTCAGCAATGAGTGA-3'

- hIL-6 forward 5'-TAATGGGCATTCCTTCTTCT-3' hIL-6 reverse 5'-TGTCCTAACGCTCATACTTTT-3'

- hlL-10 forward 5'-TTATCTTGTCTCTGGGCTTGG-3' hIL-10 reverse 5'-GTTGGGGAATGAGGTTAGGG-3'

- hNFATC1 forward 5'-TGCAAGCCGAATTCTCTTGGTGGTT-3' 
hNFATc1 reverse 5'-ACGTTGGCGGGAAGGTAGGTGAAA-3'

- hRANK forward 5'-CCATCATCTTTGGCGTTTG-3'

hRANK reverse 5'-AGCTGTGAGTGCTTTCCCT-3'

- hTREM-2 forward 5'-AGCCTCTTGGAAGGAGAAAT-3'

hTREM-2 reverse 5'-AGGAGGAGAAGGATGGAAGT-3'

- hOSCAR forward 5'-GCTGTGCCCTGTGCTGT-3'

hOSCAR reverse 5'-CCACTAATCGCGTCTTCC-3'

- hSIRP $\beta 1$ forward 5'-AGGCCACTTCCCACGGGTAACAAC-3'

hSIRP $\beta 1$ reverse 5'-AGTAGTAGGTGCCGGCGTCTGCT-3'

- hILT7 forward 5'-GTGGCTGGCTTGGTCCTGCTGTTC-3'

hILT7 reverse 5'-CTGTTCCCAAGGCTCCACCACTCT-3'

- hDAP12 forward 5'-CCTCTCCTGCTGGCTGTAAG-3'

hDAP12 reverse 5'-GCAACTGCAATCGCTCTGG-3'

- hFcR $\gamma$ forward 5'-ATGCCATCCTGTTTCTGTAT-3'

hFcR $\gamma$ reverse 5'-AAGTCTCCTGGTTCCTGGTGCTCA-3'

- hCathepsin K forward 5'-CTCTTCCATTTCTTCCACGAT-3'

hCathepsin $\mathrm{K}$ reverse 5'-ACACCAACTCCCTTCCAAAG-3'

- hintegrin $\beta 3$ forward 5'-GGAAGAACGCGCCAGAGCAAAATG-3'

hIntegrin 33 reverse 5'-CCCCAAATCCCTCCCCACAAATAC-3'

- mGAPDH forward 5'-ATCAAGAAGGTGGTGAAGCA-3'

mGAPDH reverse 5'-AGACAACCTGGTCCTCAGTGT-3'

- mSOCS1 forward 5'-CATCCCTCTTAACCCGGTAC-3'

mSOCS1 reverse 5'-TGGAAGGGGAAGGAACTCAG-3' 
- mSOCS3 forward 5'-ATTTGCCTCAATCACTTTTAT-3' mSOCS3 reverse 5'-ACTGGGATTTGGTTGAGTTT-3'

- mIRF-1 forward 5'-CCCACAGAAGAGCATAGCAC-3' mIRF-1 reverse 5'-AGCAGTTCTTTGGGAATAGG-3'

- mIP-10 forward 5'-ATTCTTTAAGGGCTGGTCTGA-3' mIP-10 reverse 5'-CACCTCCACATAGCTTACAGT-3'

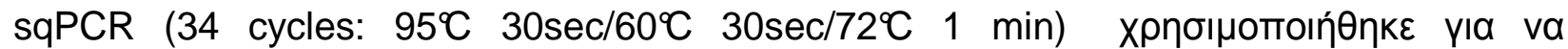

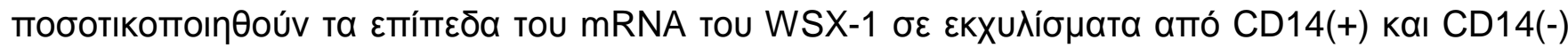

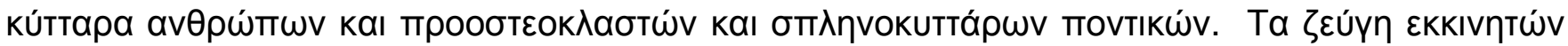

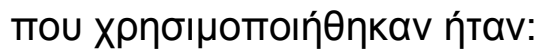

- hWSX-1 forward 5'-GGAATTACCTGCTGCTGCTC-3' hWSX-1 reverse 5'-GAGGCTGAATCCAAGCAGAC-3'

- mWSX-1 forward 5'-CAAGAAGAGGTCCCGTGCTG-3' mWSX-1 reverse 5'-TTGAGCCCAGTCCACCACAT-3' 


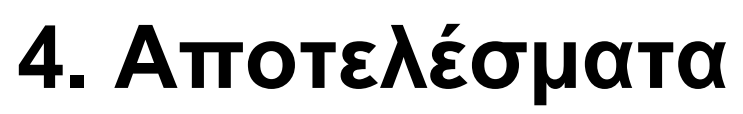




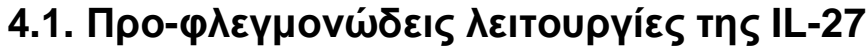

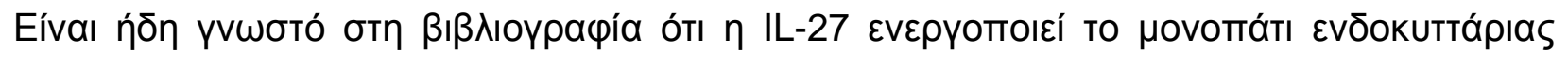

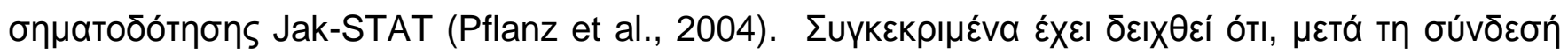

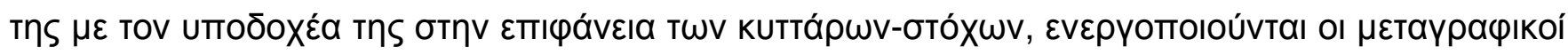

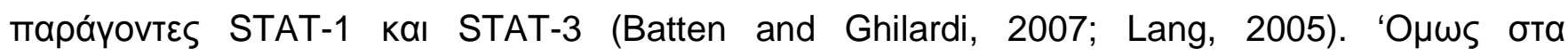

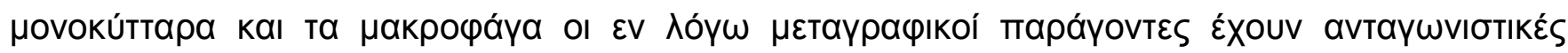

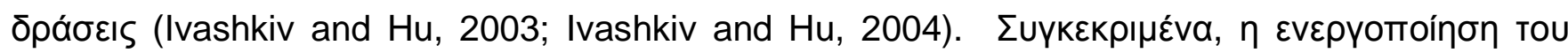

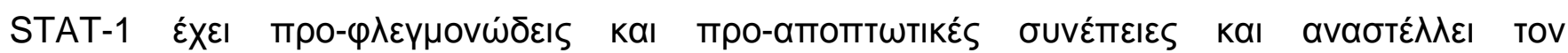

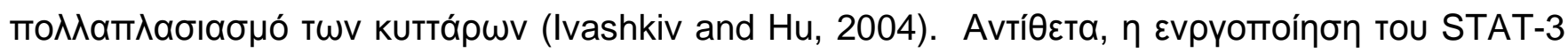

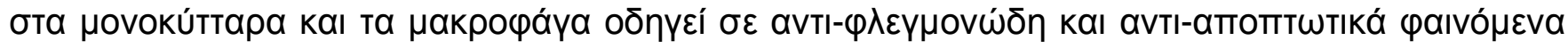

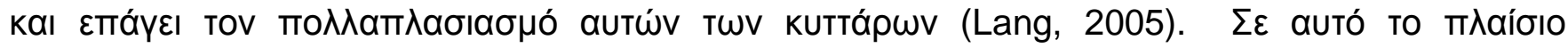

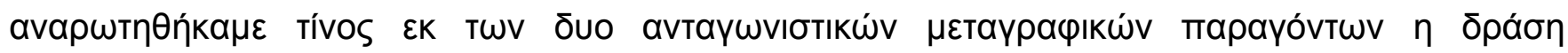

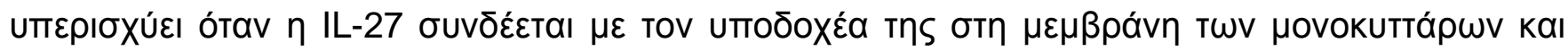

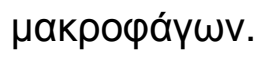

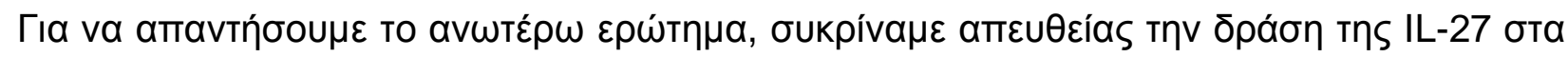

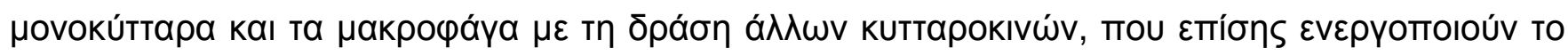

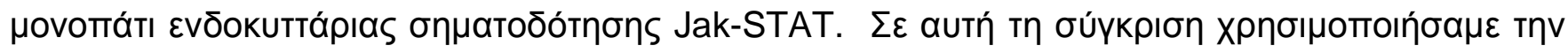

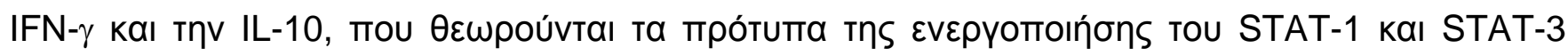

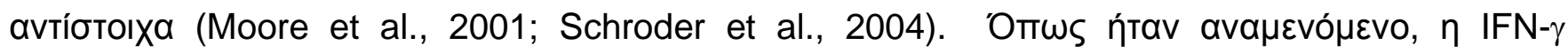

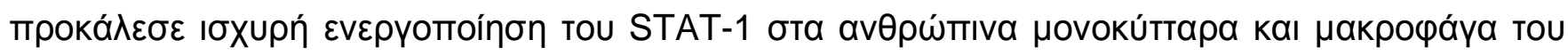

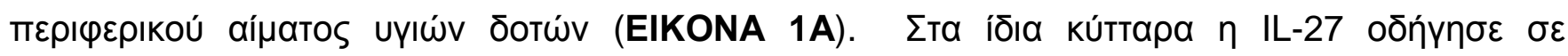

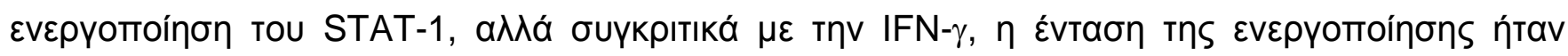

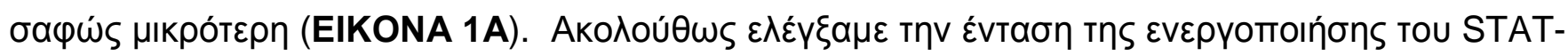




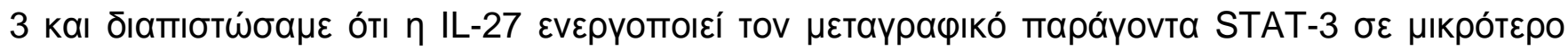

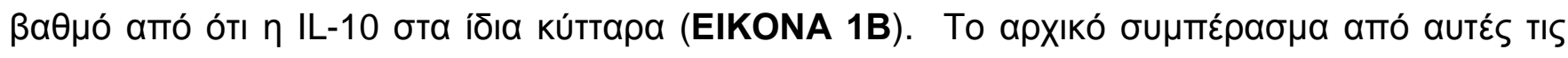

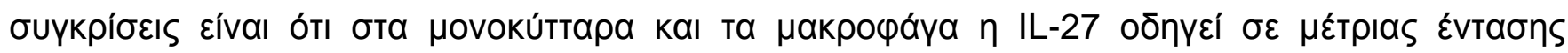

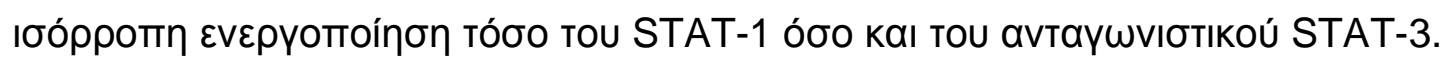

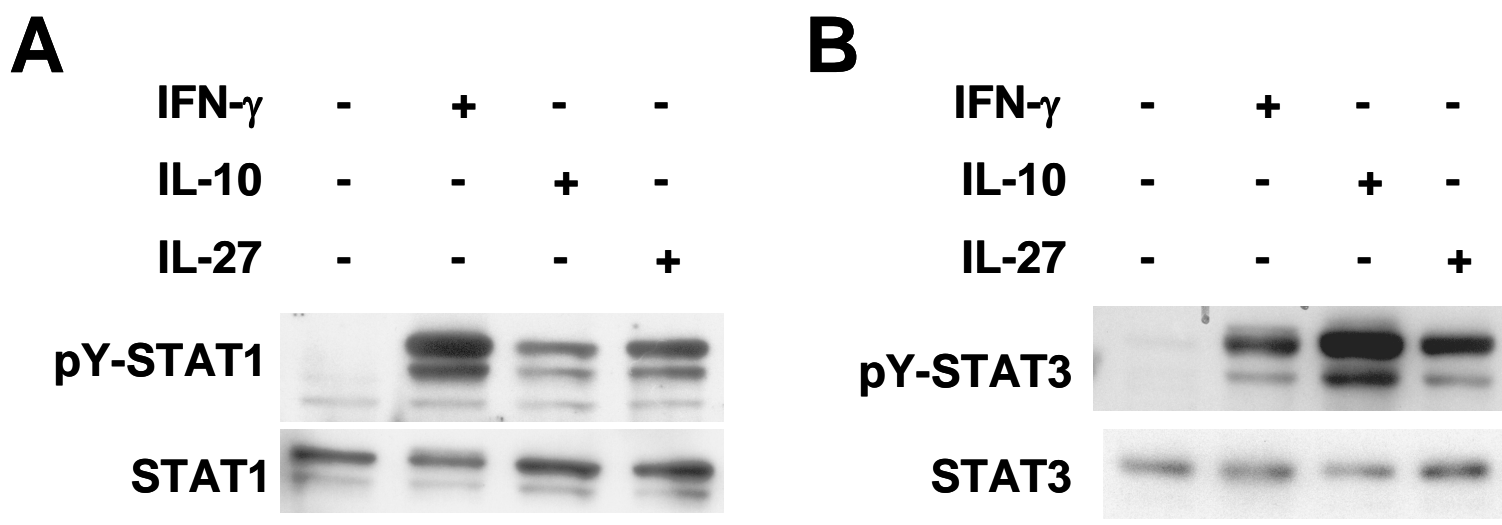

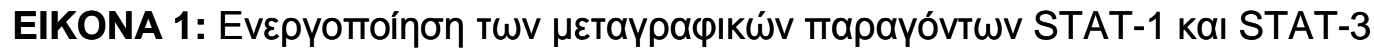

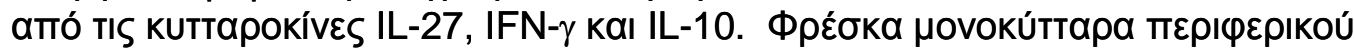

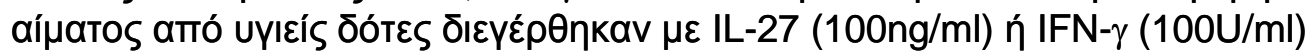

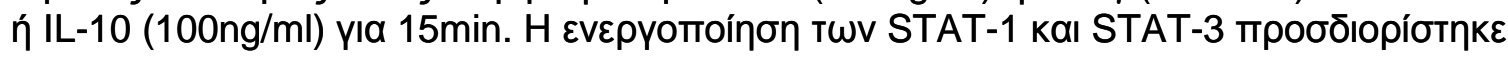

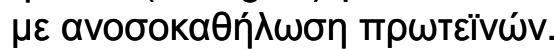

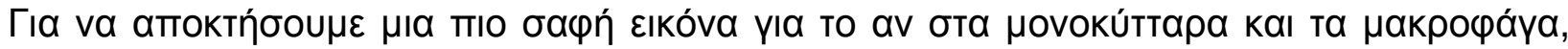

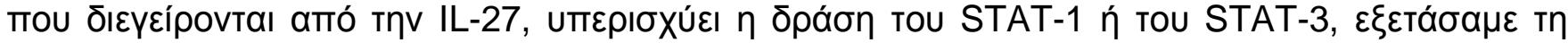

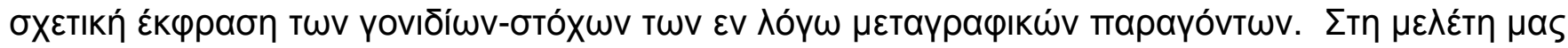

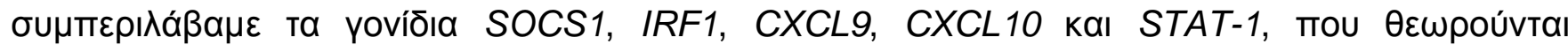

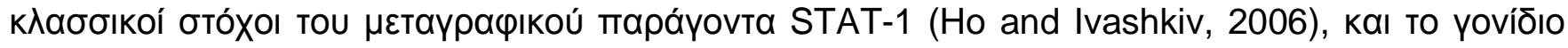

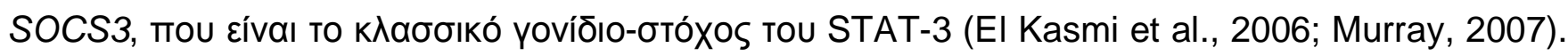

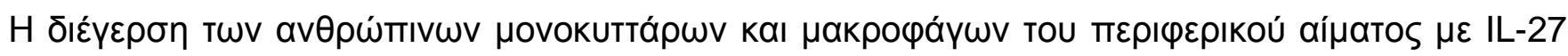

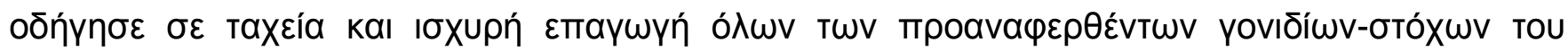

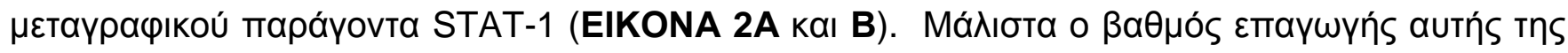

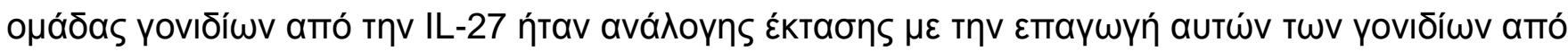




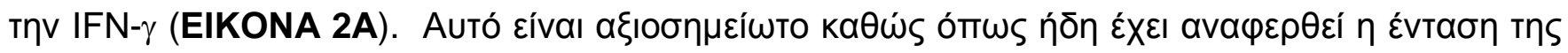

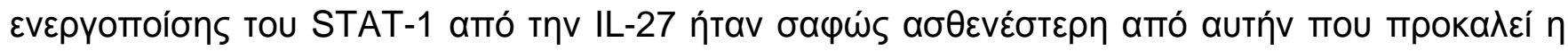

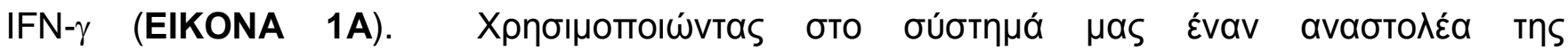

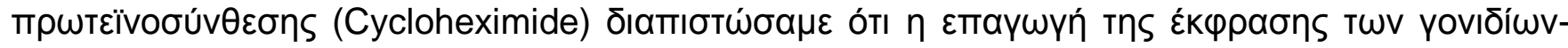

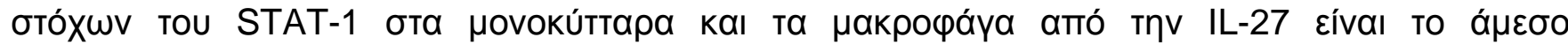

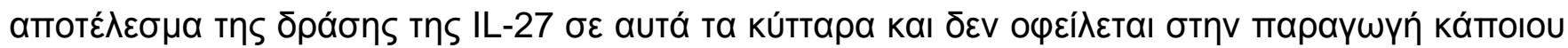

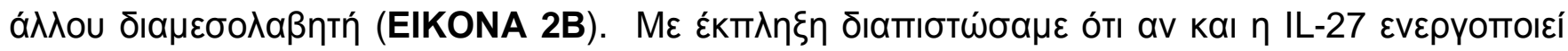

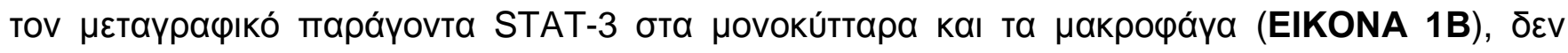

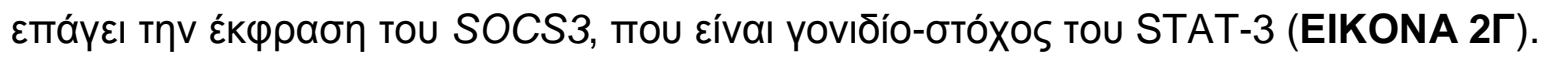
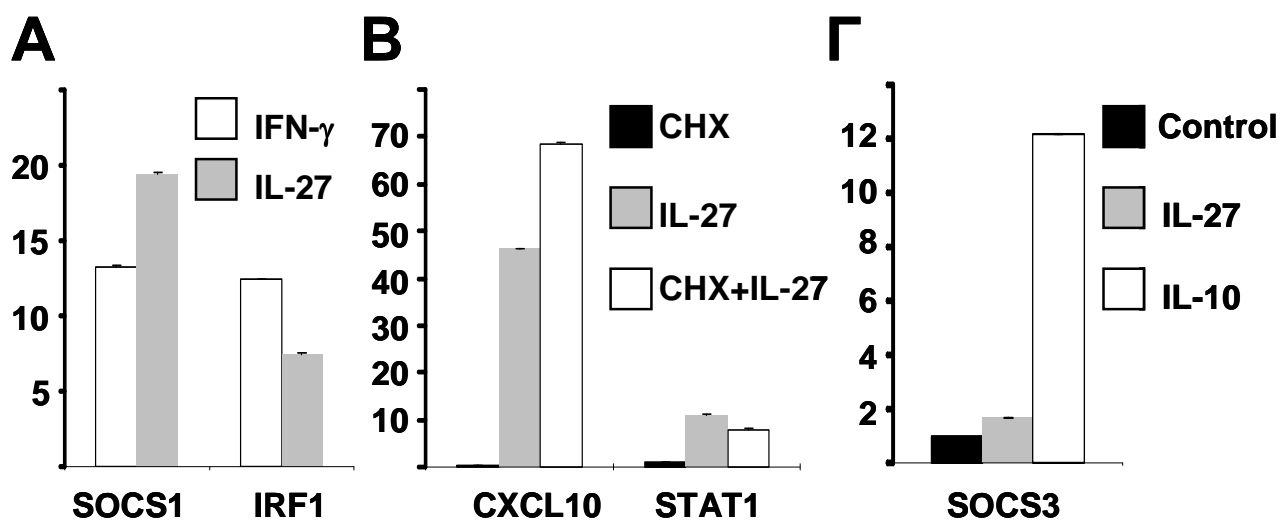

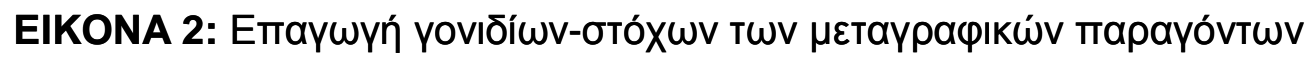

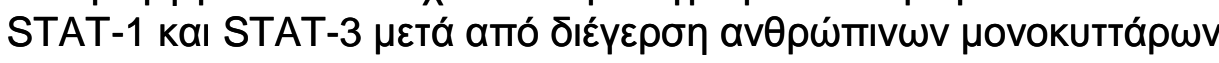

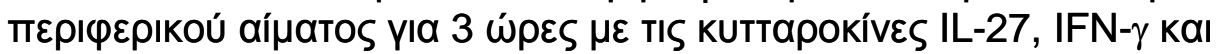

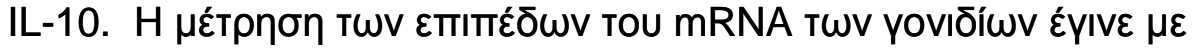
qPCR.

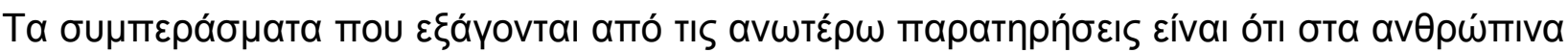

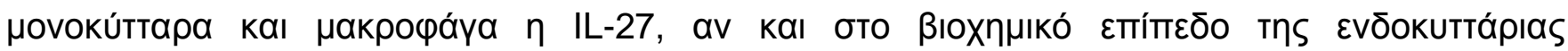

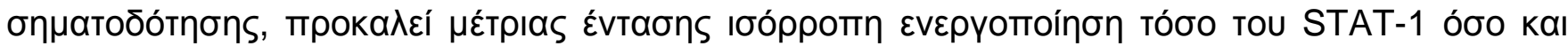




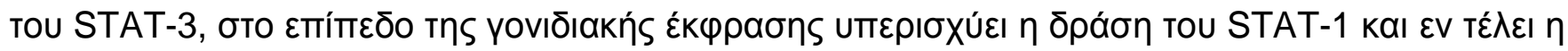

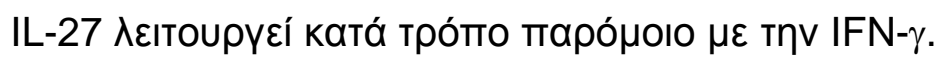




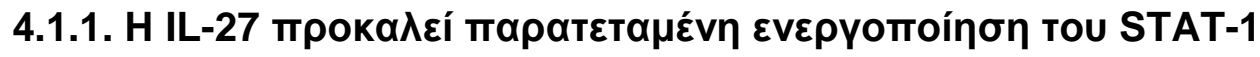

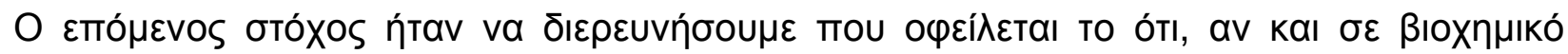

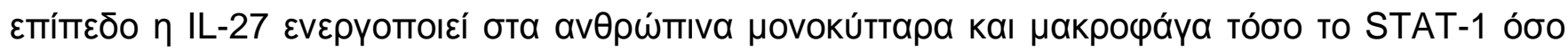

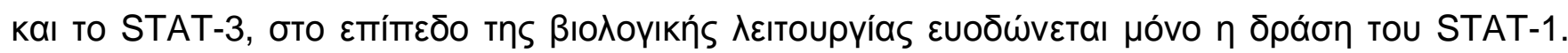

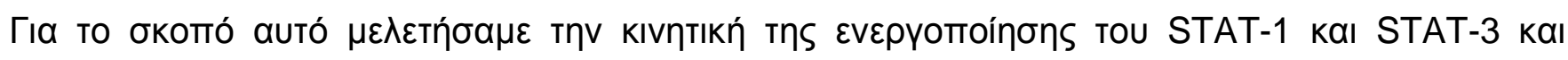

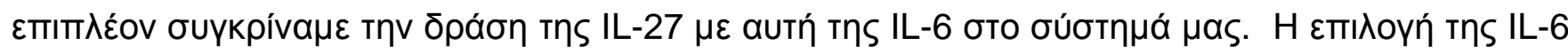

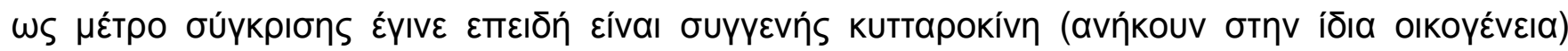

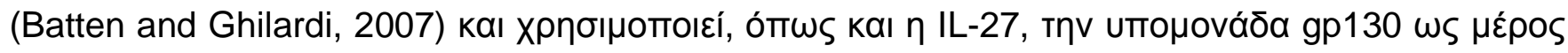

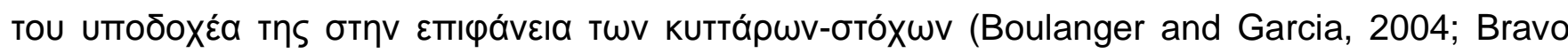
and Heath, 2000; Chow et al., 2002; Fukada et al., 1999; Heinrich et al., 1998; Kamimura et al., 2003; Murakami et al., 2004; Scheller and Rose-John, 2006; Schmitz et al., 2000).

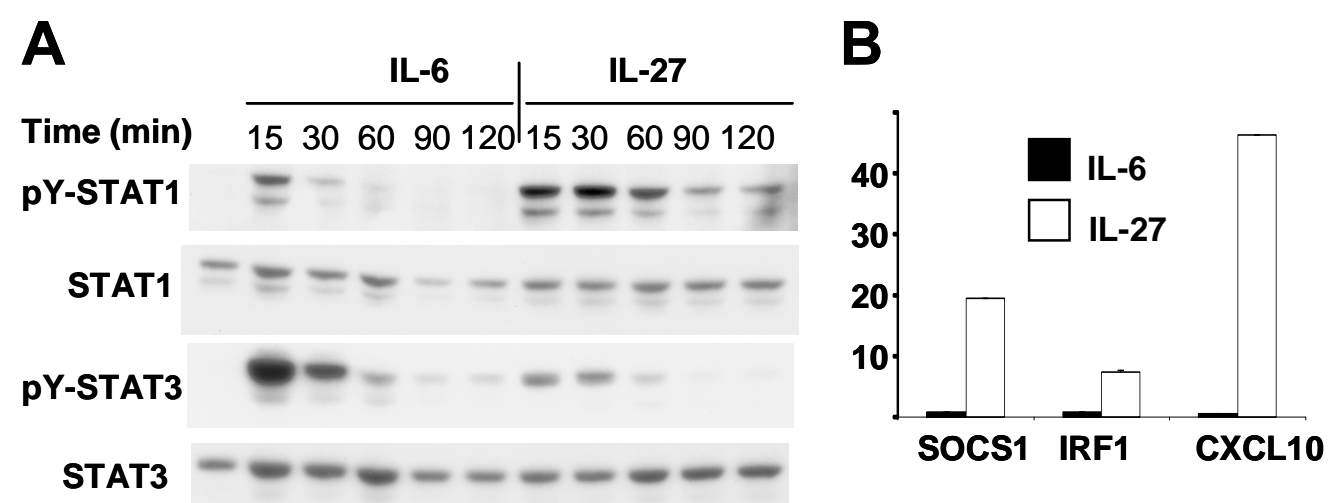

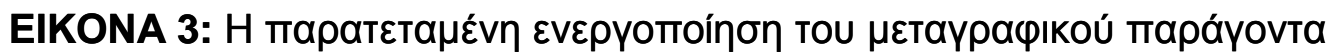

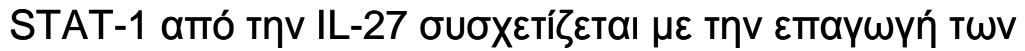

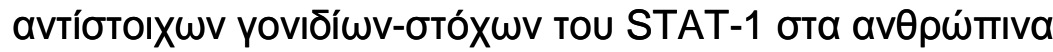

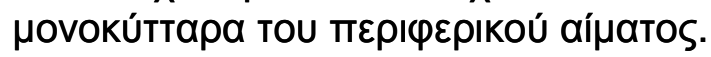

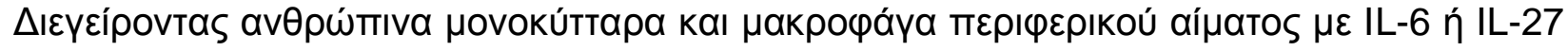

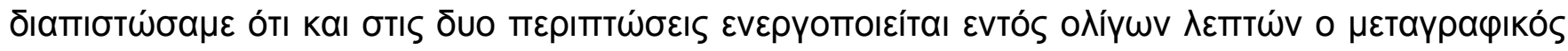

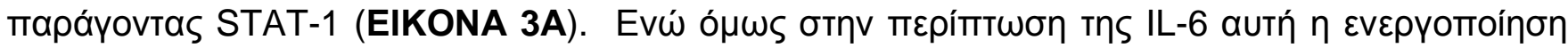




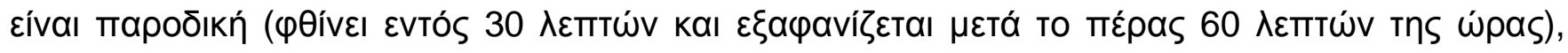

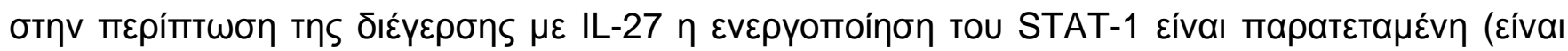

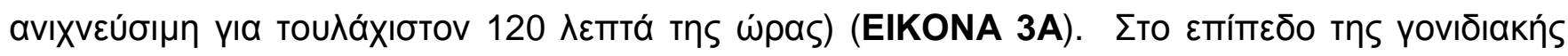

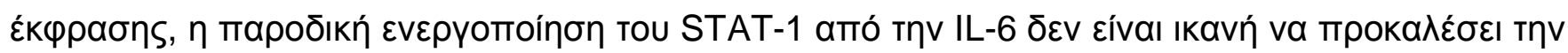

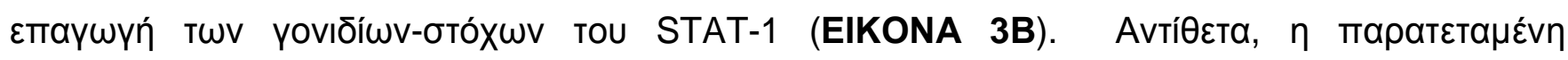

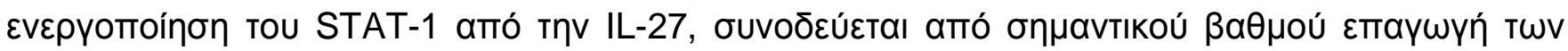

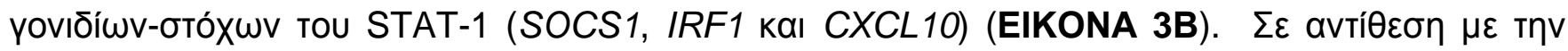

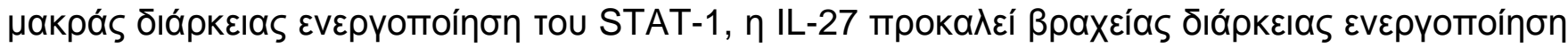

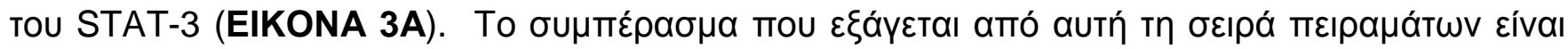

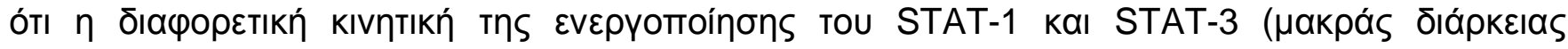

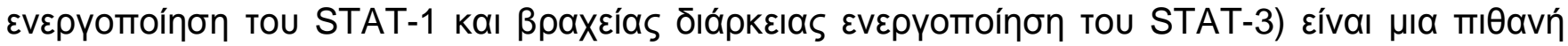

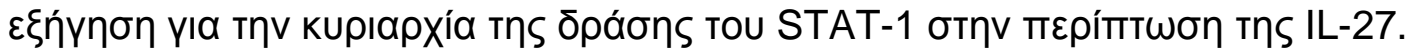




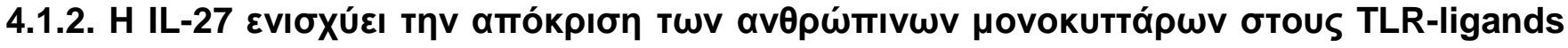

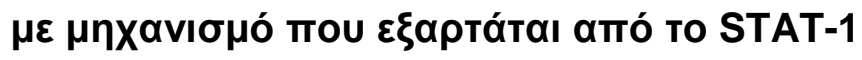

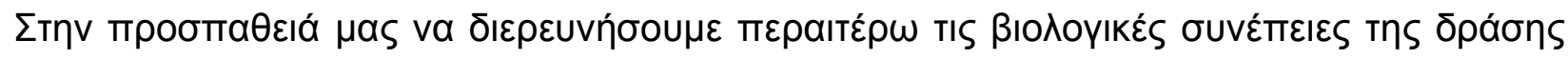

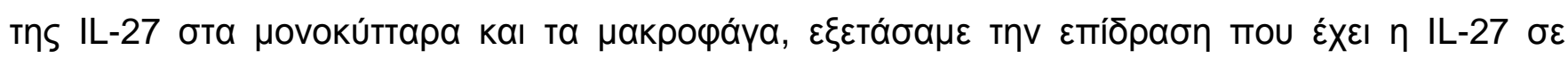

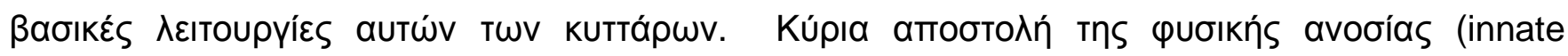

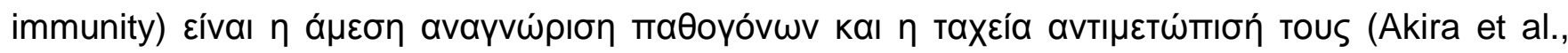
2006; Janeway and Medzhitov, 1998; Medzhitov and Janeway, 1998a; Medzhitov and Janeway,

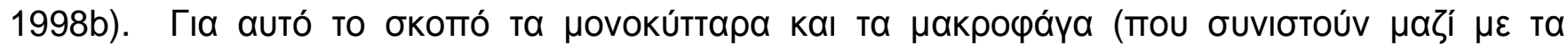

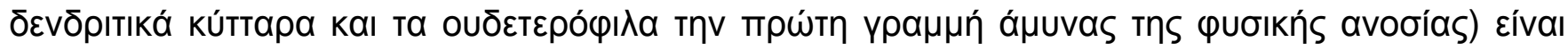

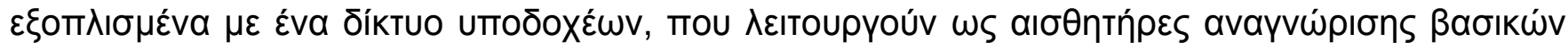

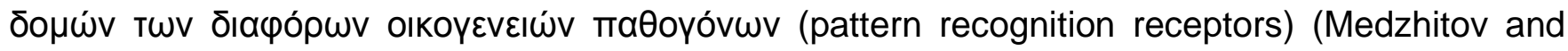

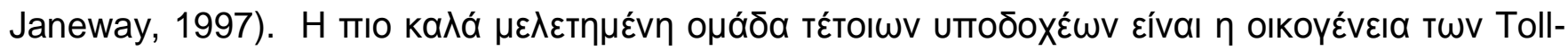

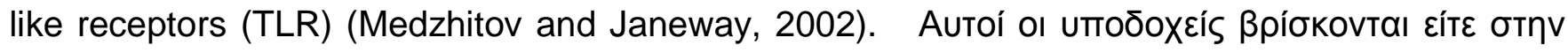

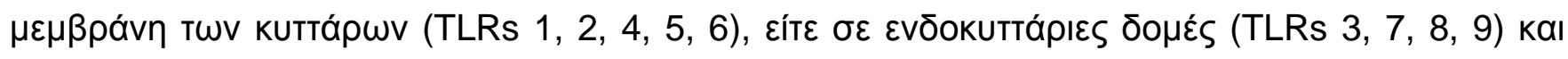

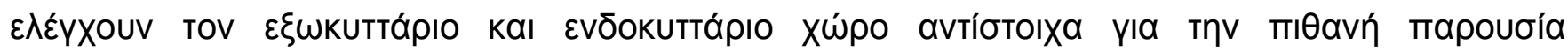

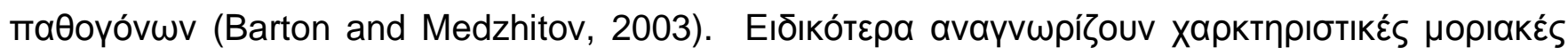

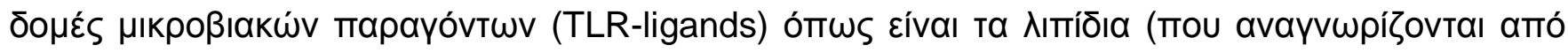

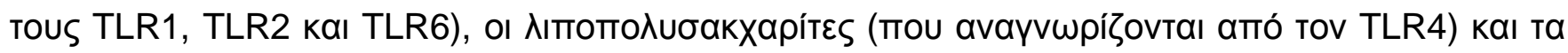

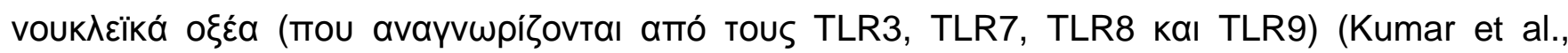
2009).

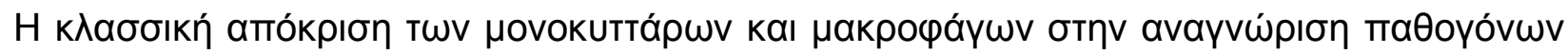

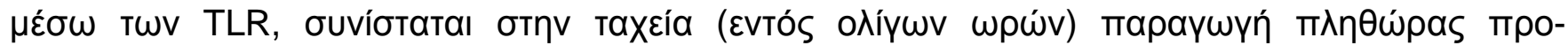

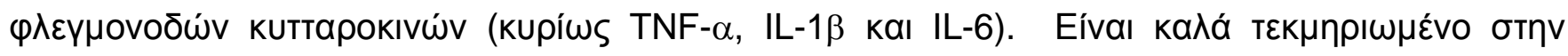




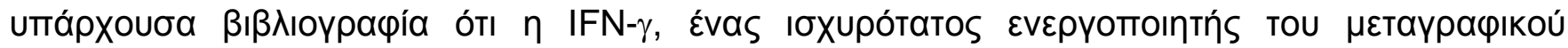

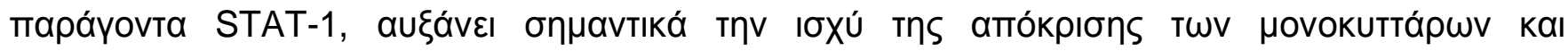

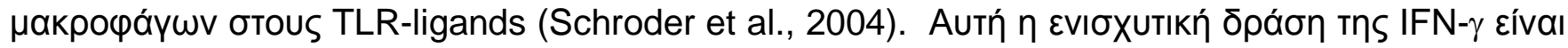
үvwotń ws priming.
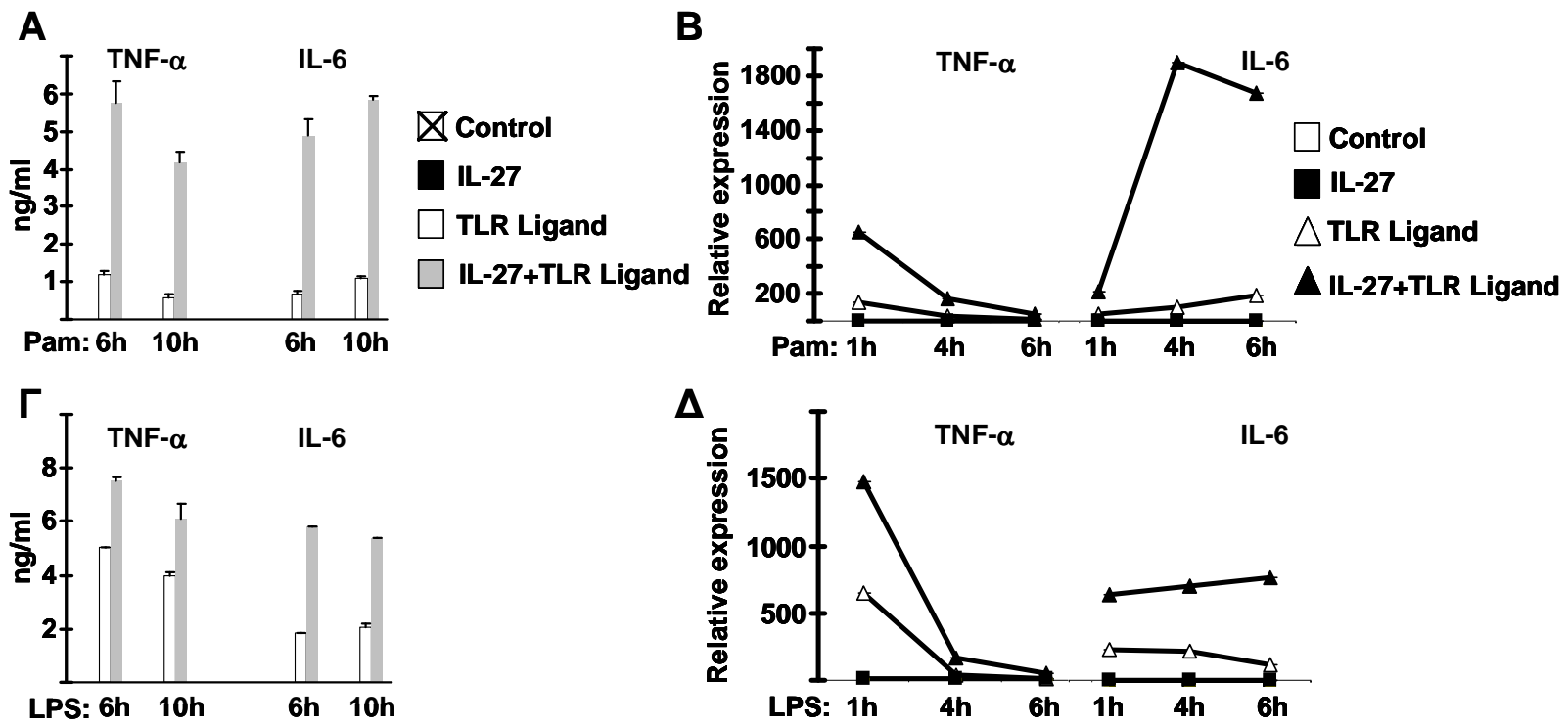

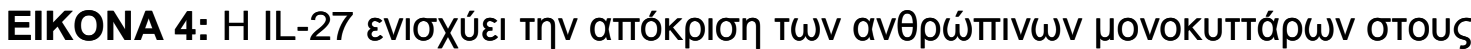
TLR-ligands

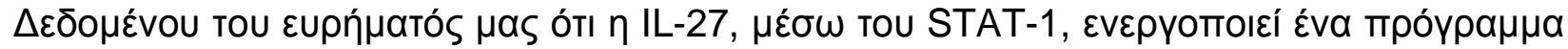

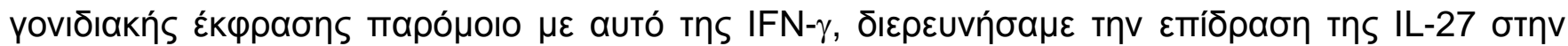

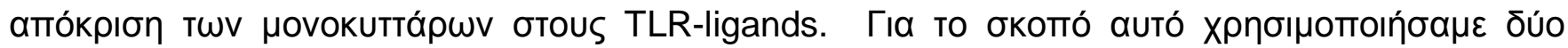

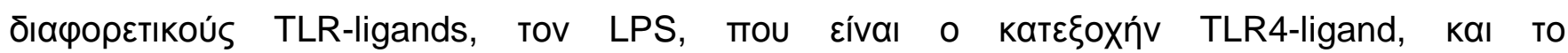

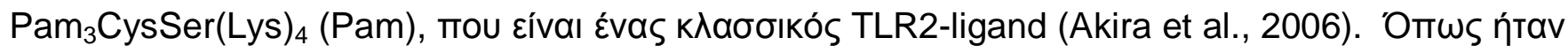

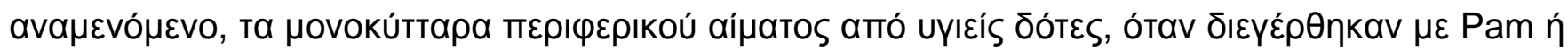

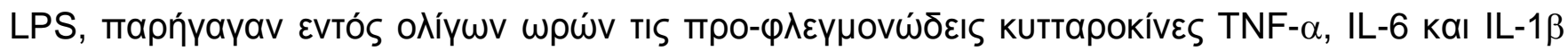

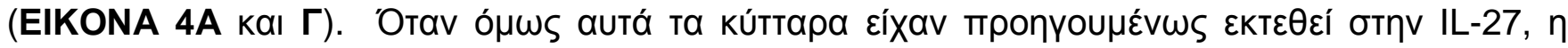




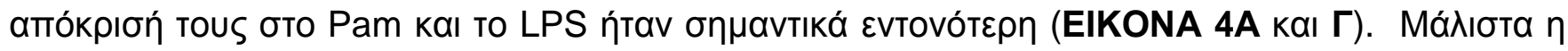

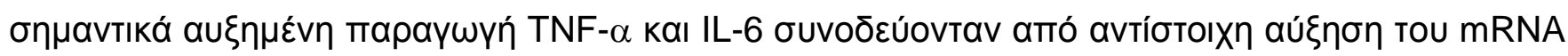

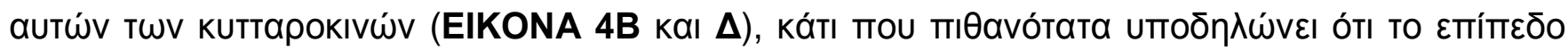

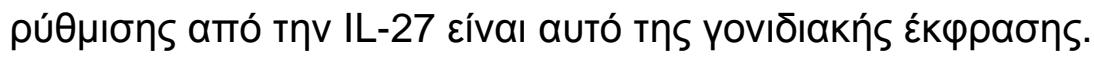

A

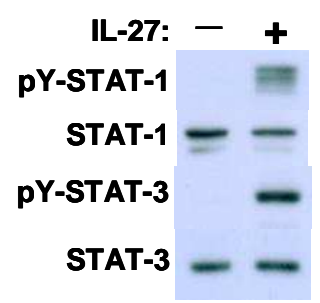

B

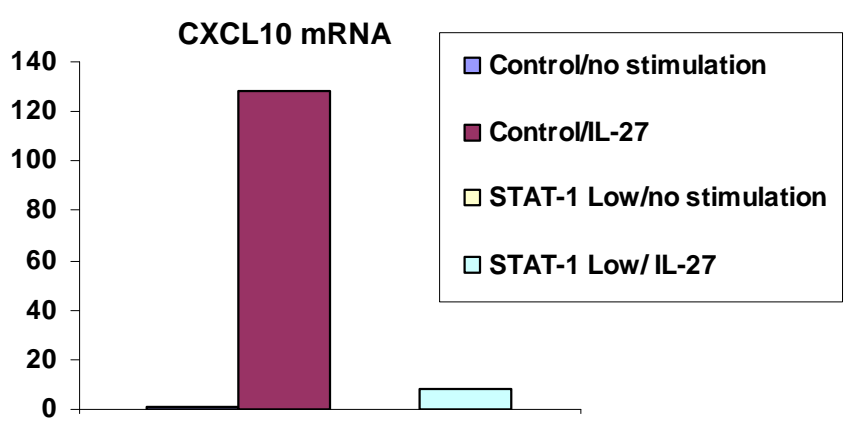

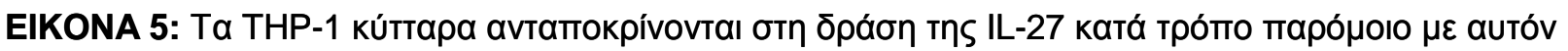

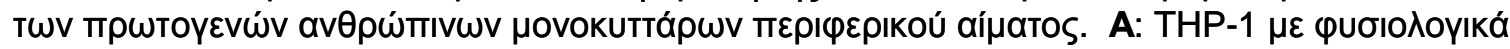

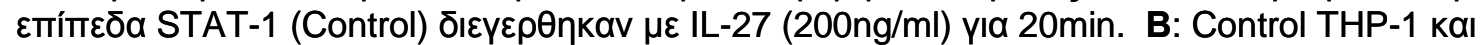

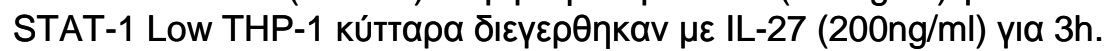

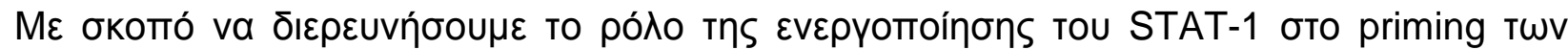

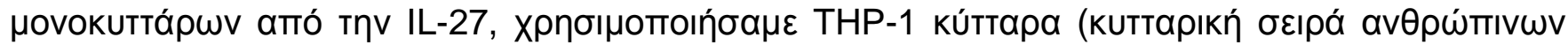

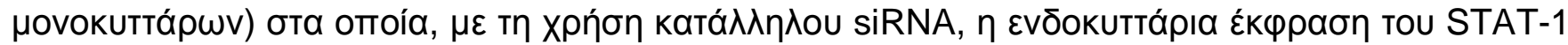

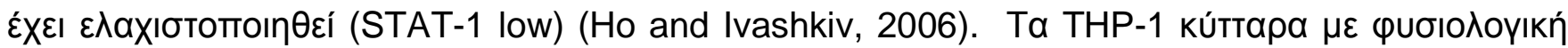

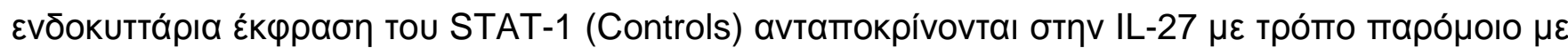

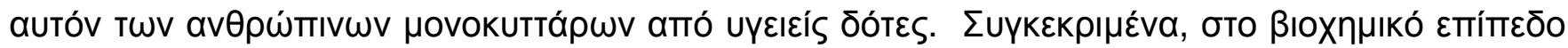

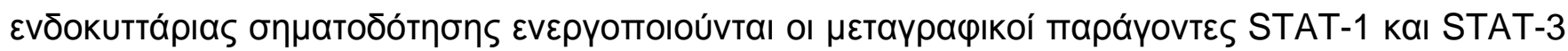

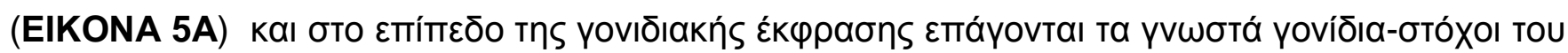
STAT-1 (SOCS1, IRF1, CXCL9, CXCL10 каI STAT-1) (EIKONA 5B). Avtí日Eta, ota STAT-1 low

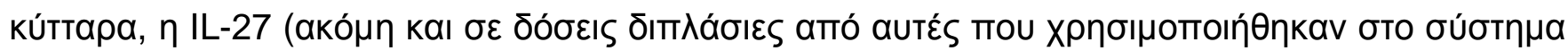

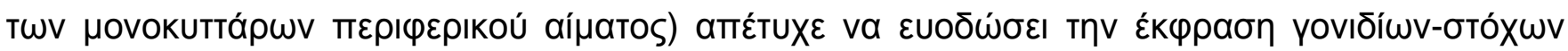
TOU STAT-1 (EIKONA 5B). 


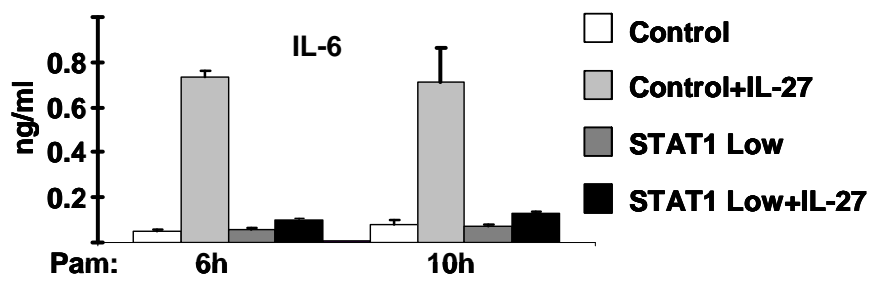

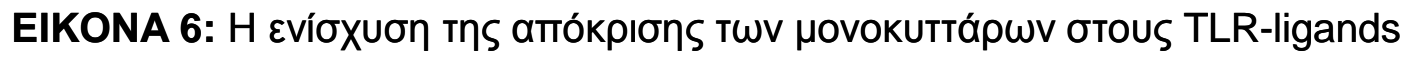

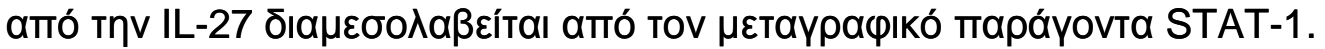

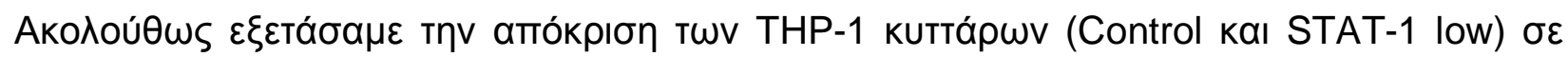

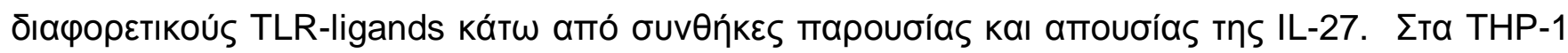

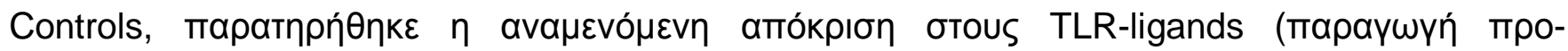

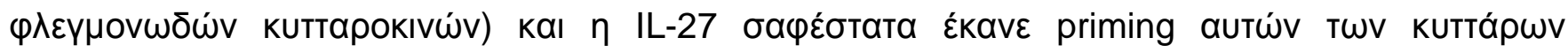

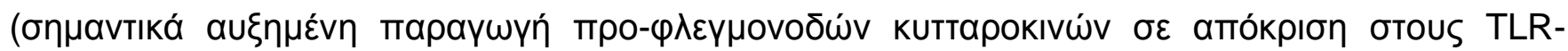

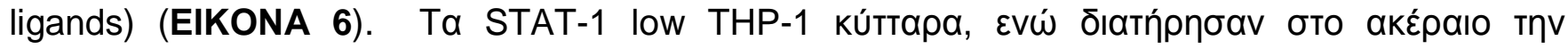

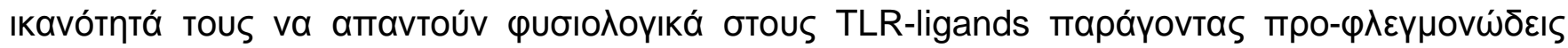

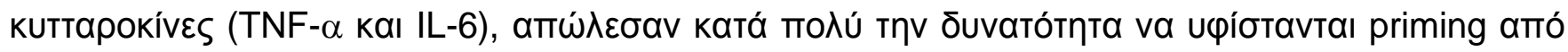

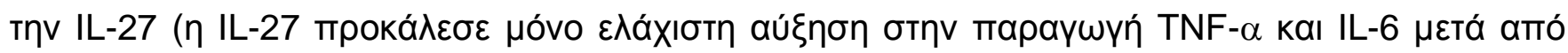

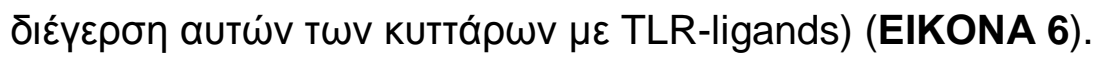

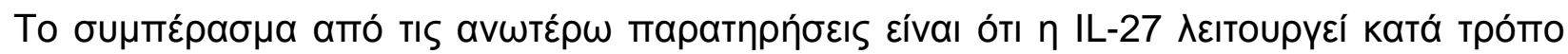

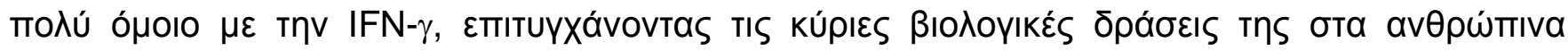

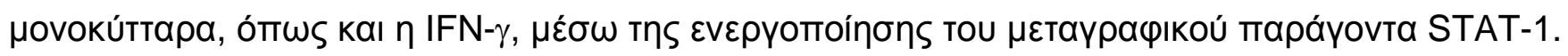




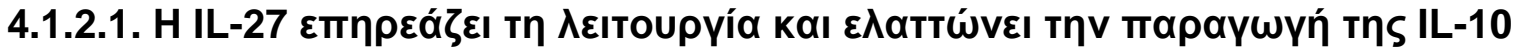

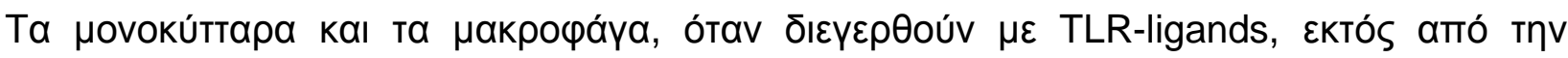

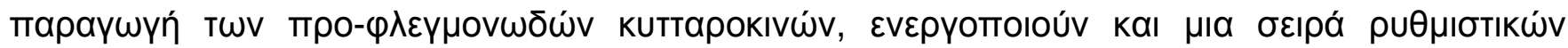

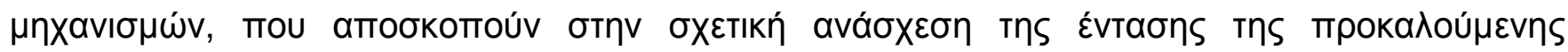

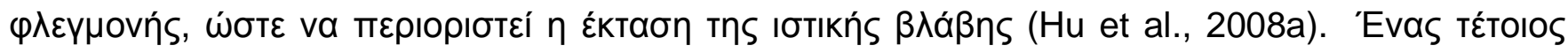

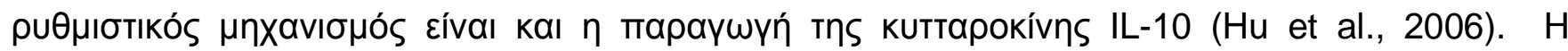

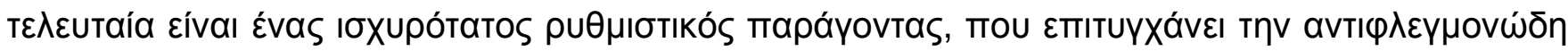

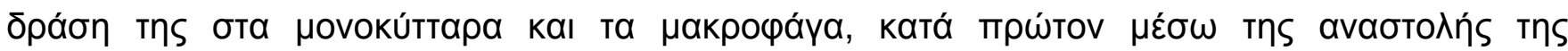

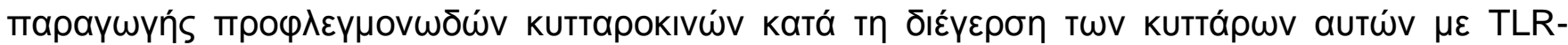

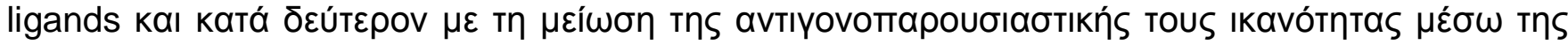

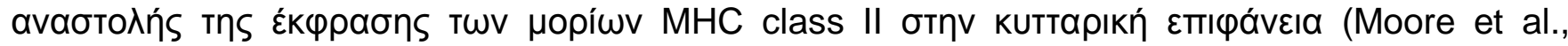

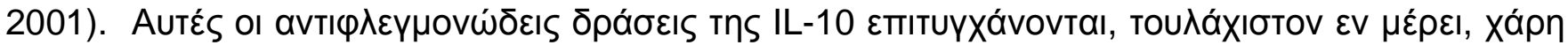

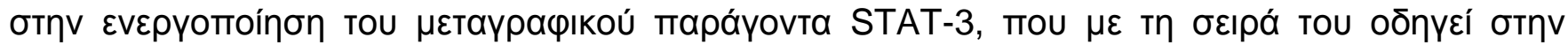

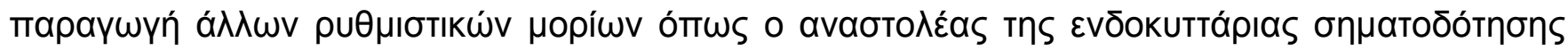

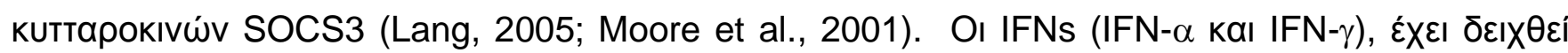

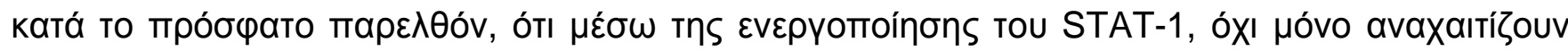

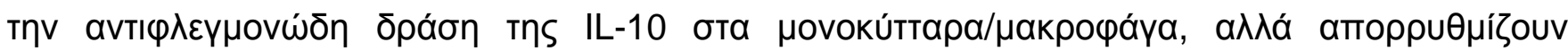

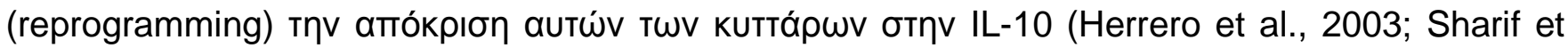

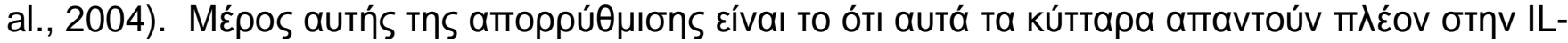

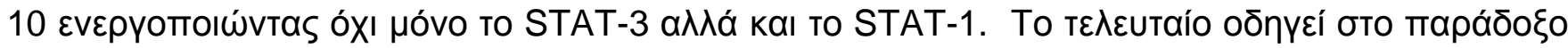

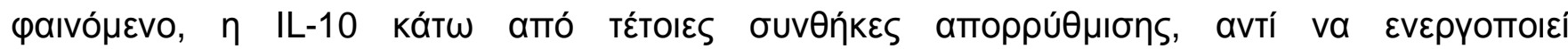

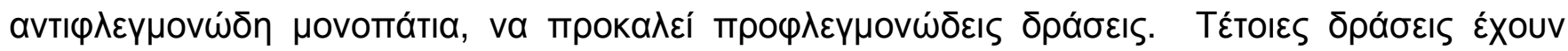

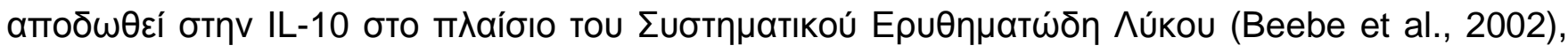




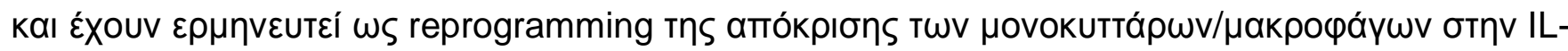

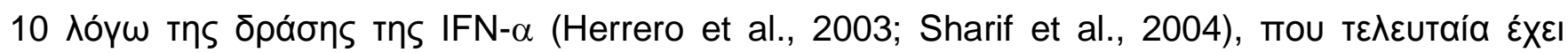

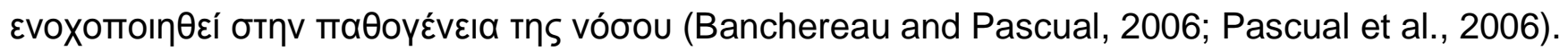
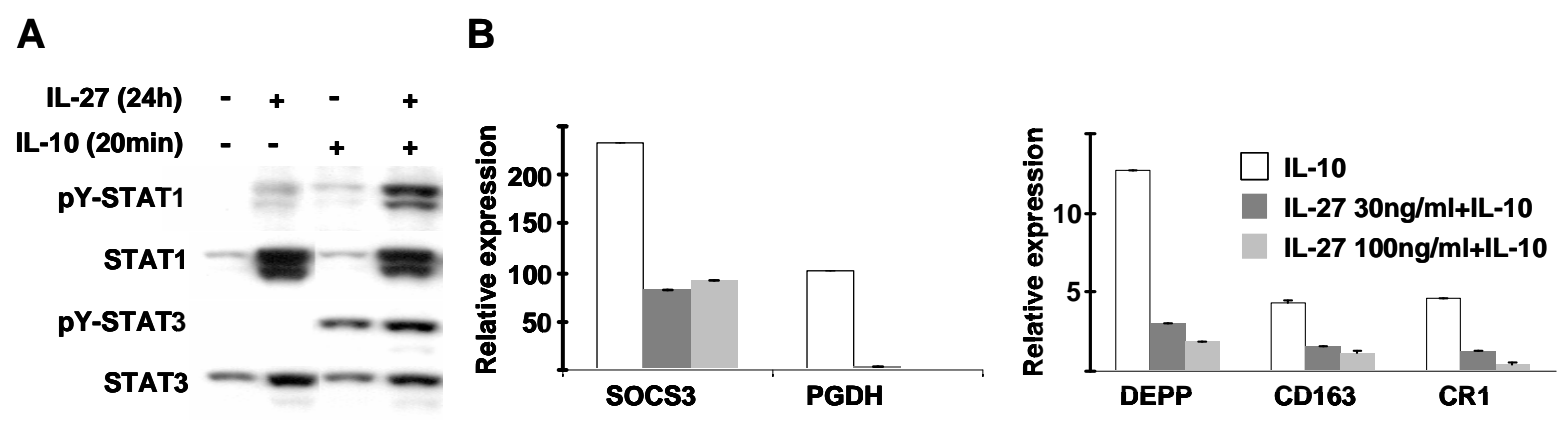

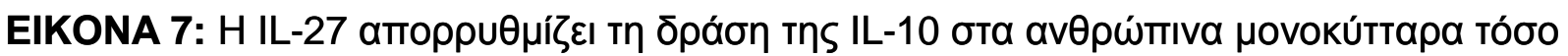

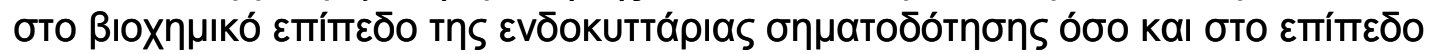

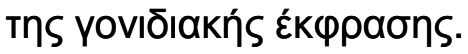

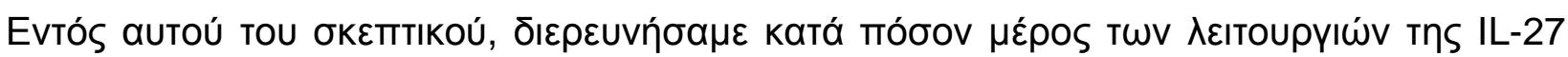

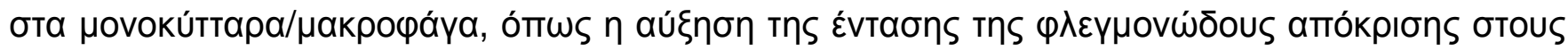

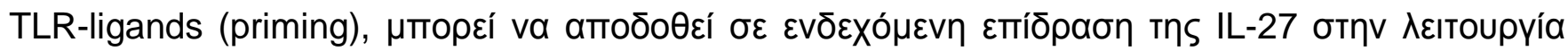

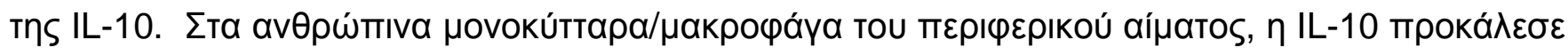

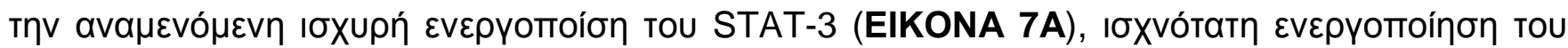

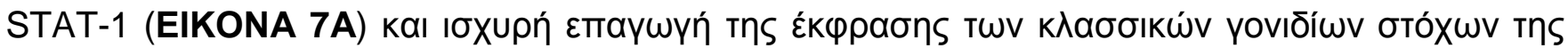
IL-10 (SOCS3, PGDH, DEPP, CD163 kaı CR1) (EIKONA 7B). 'Otav autá ta kúttapa kíxav

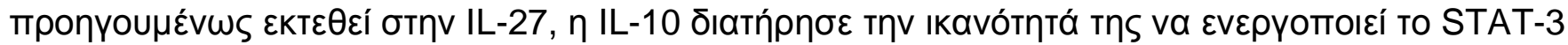

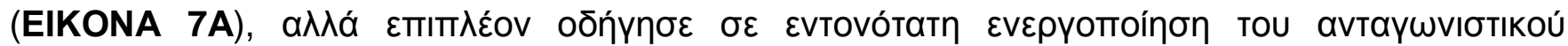

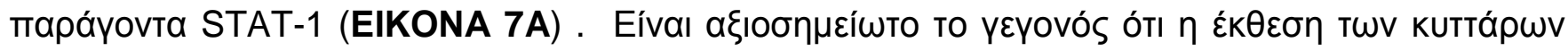

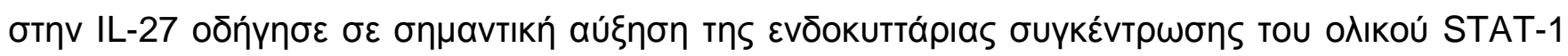

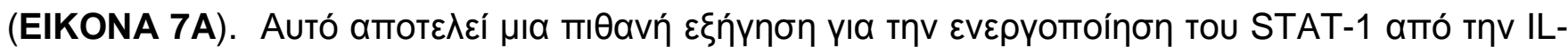




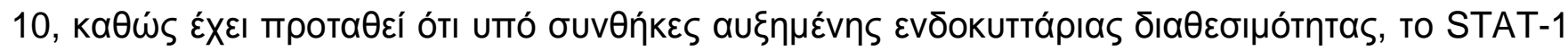

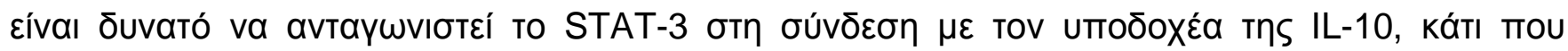

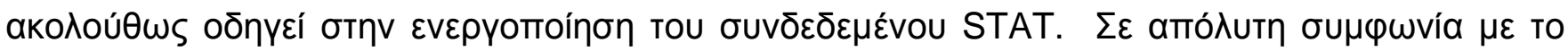

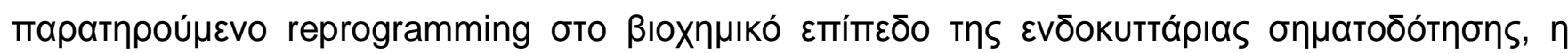

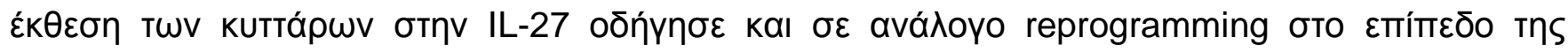

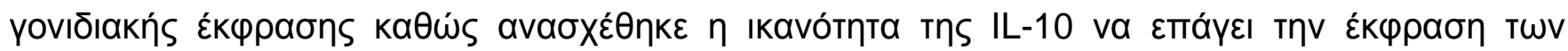

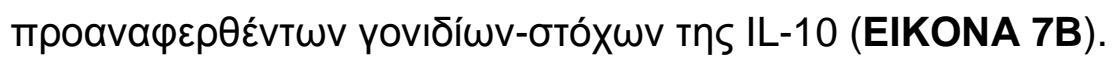
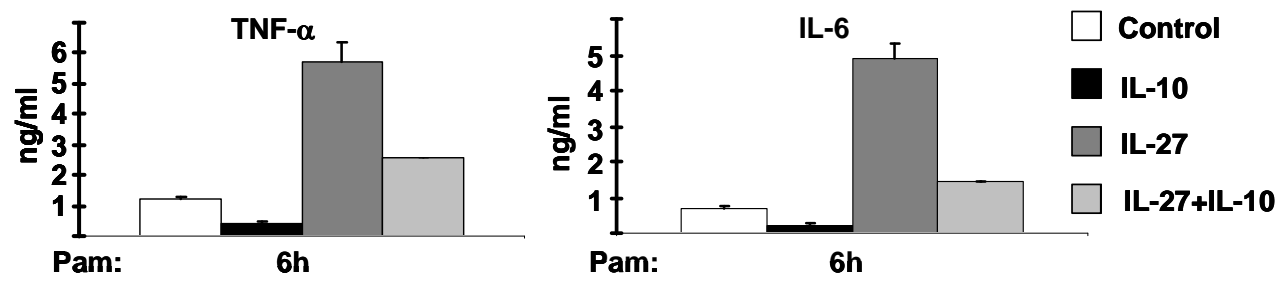

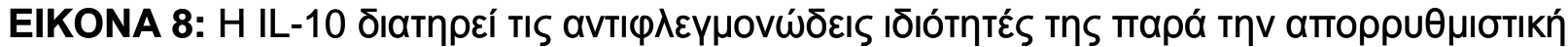

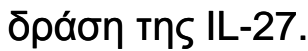

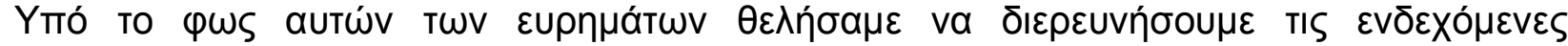

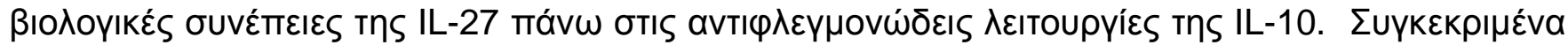

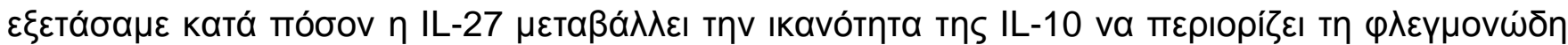

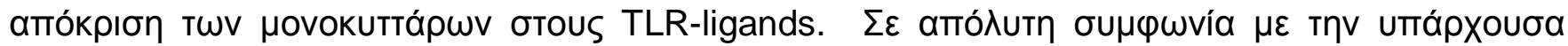

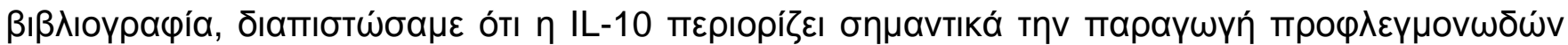

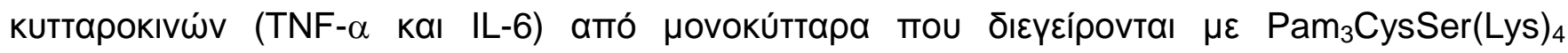

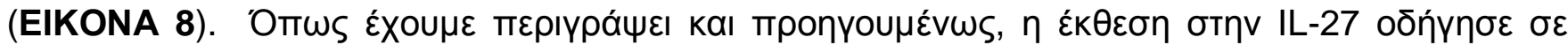

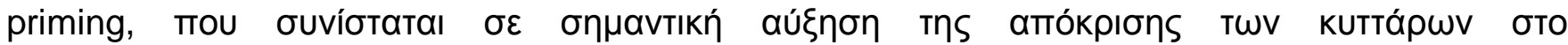

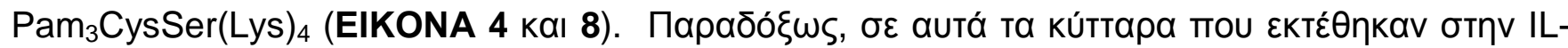

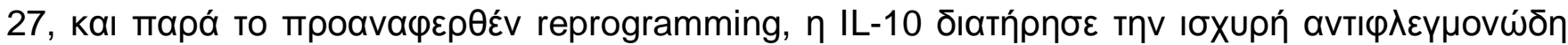




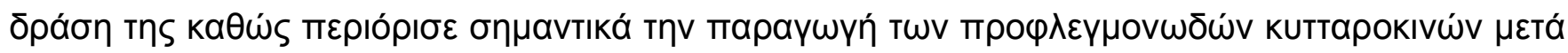

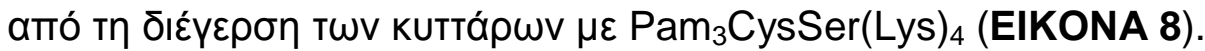

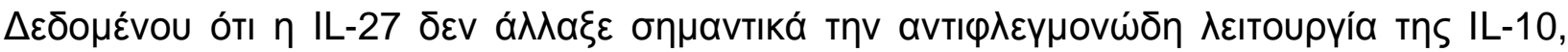

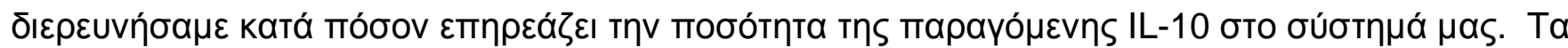

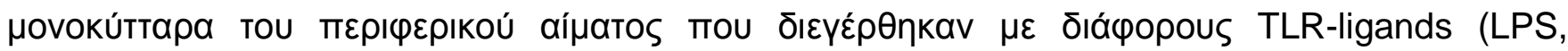

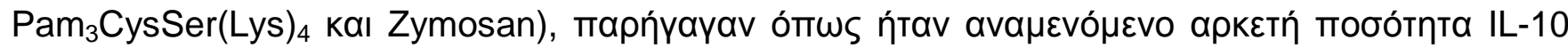
(EIKONA 9A, 9Г кवI 9E).

A
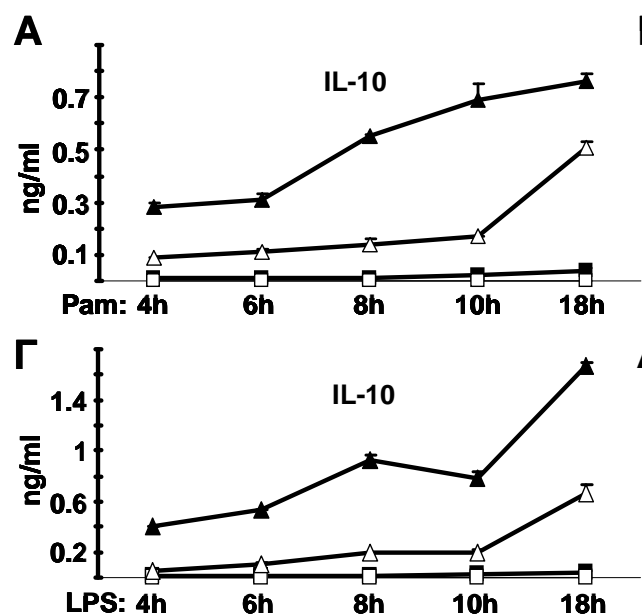

E

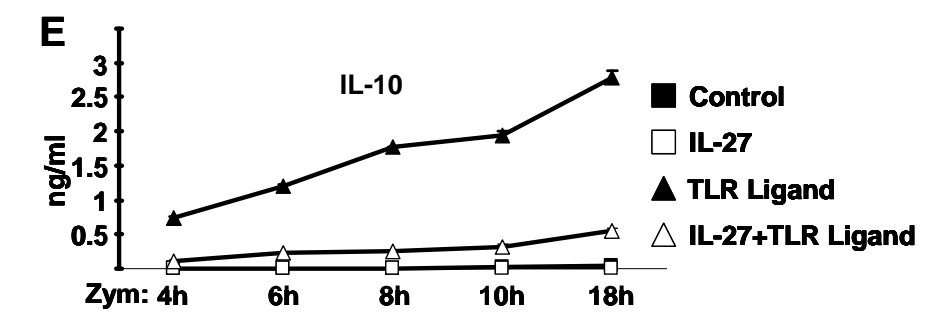

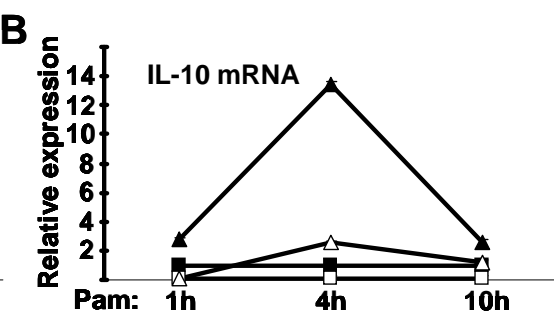

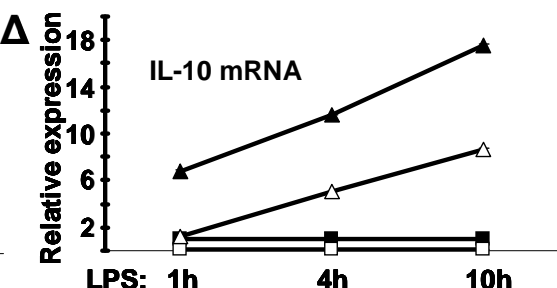

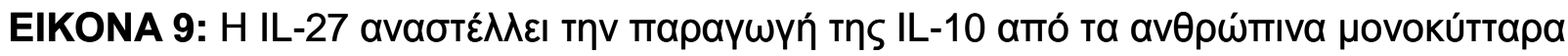

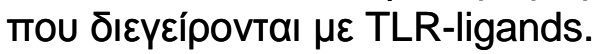

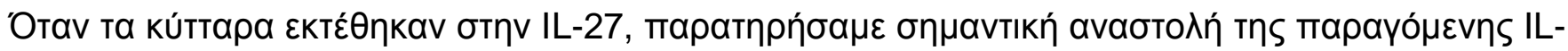

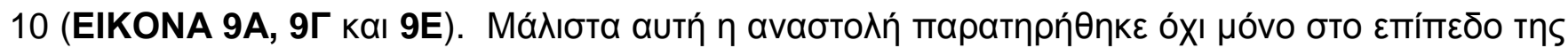

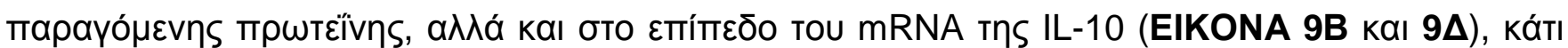

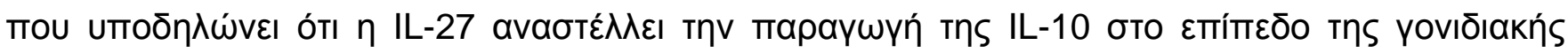




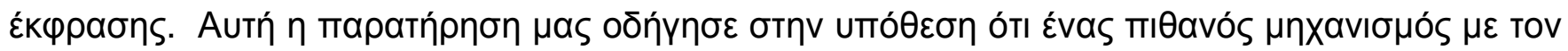

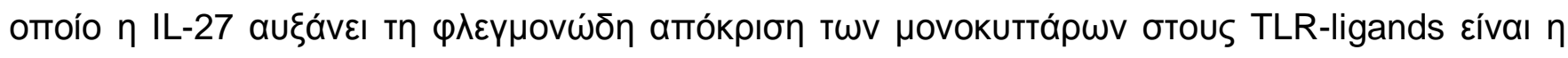

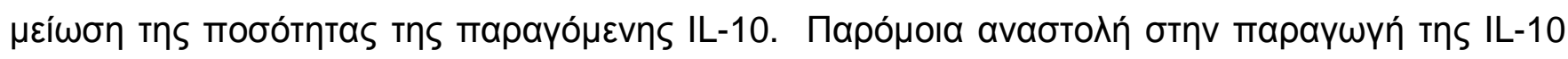

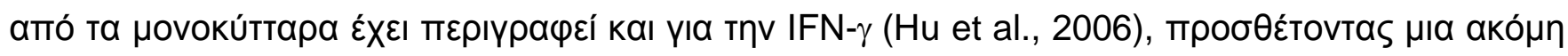

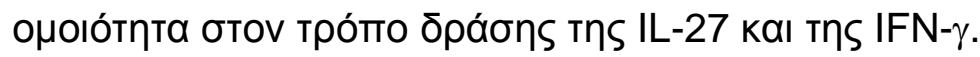




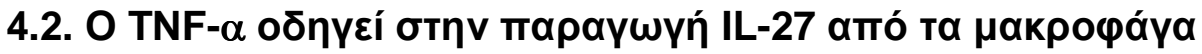

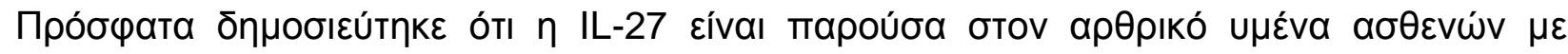

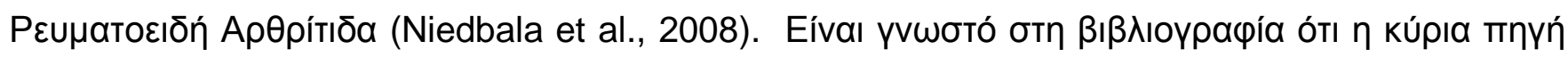

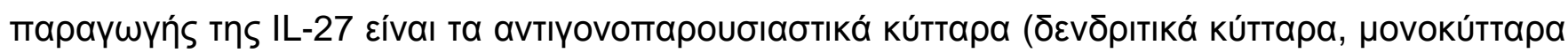

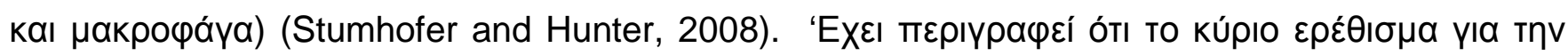

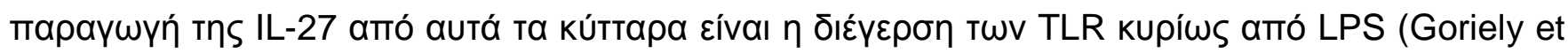
al., 2008; Liu et al., 2007; Molle et al., 2007; Wirtz et al., 2005; Yoshida et al., 2009). O TLR

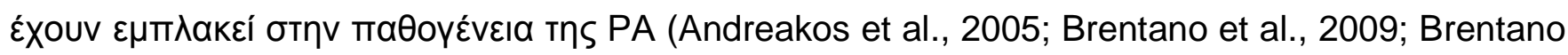

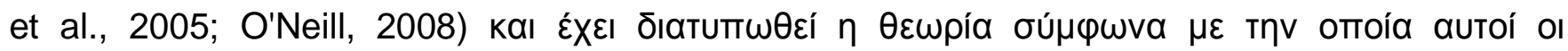

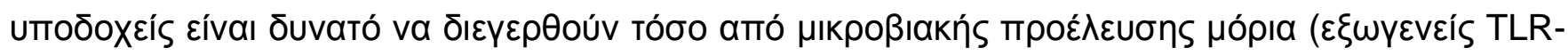

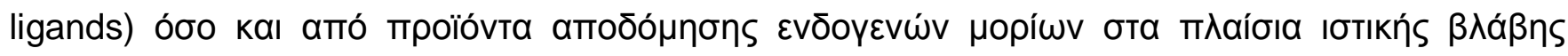

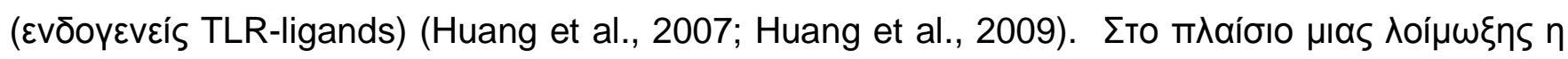

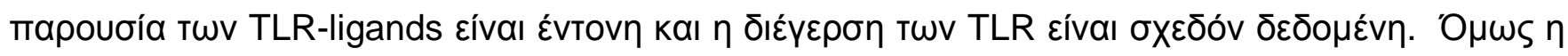

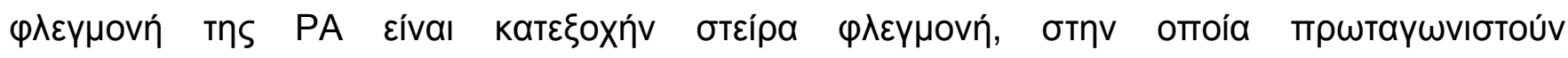

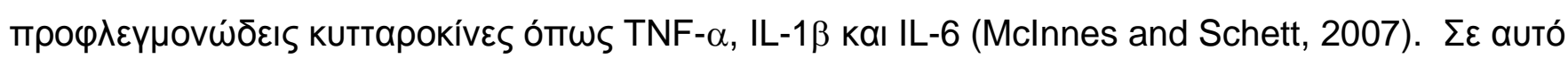

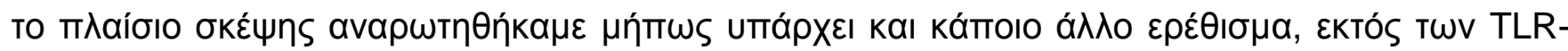

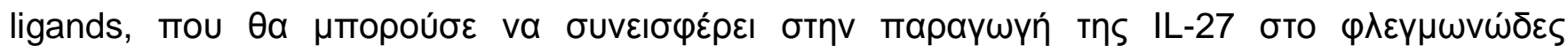

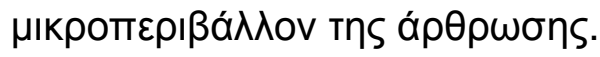


A

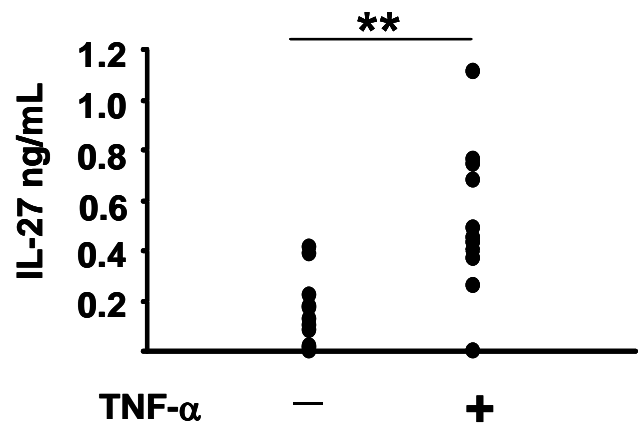

B

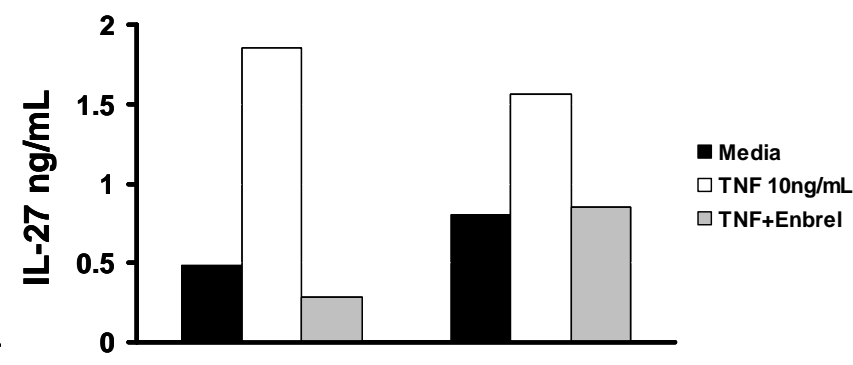

Dótns A
$\Delta$ ótns B

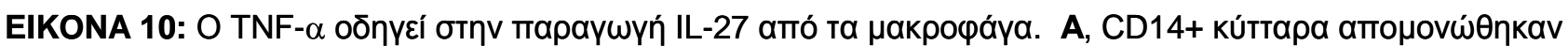

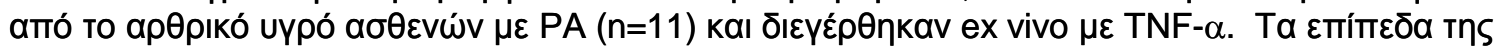

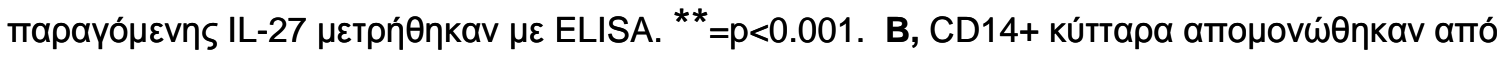

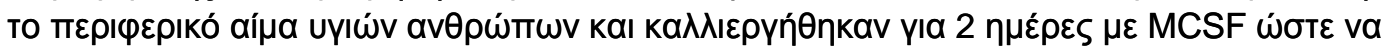

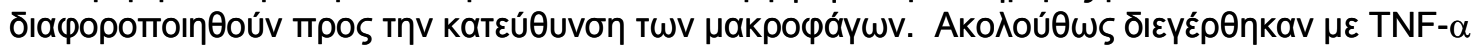

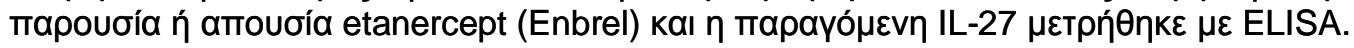

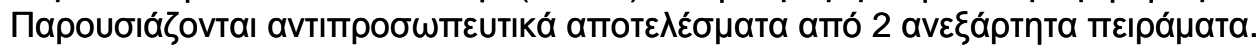

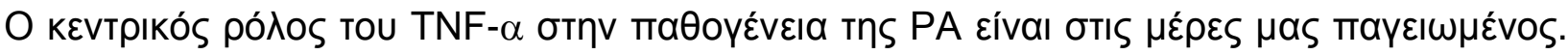

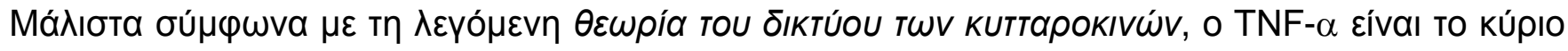

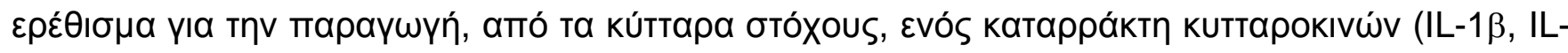

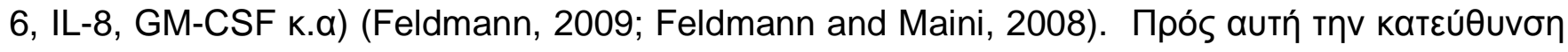

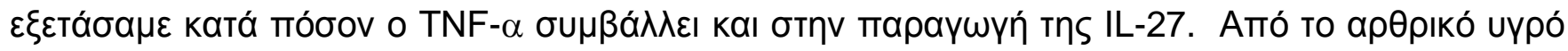

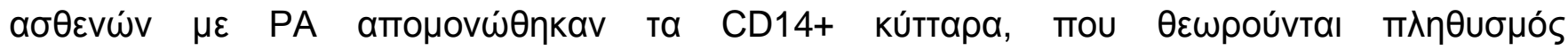

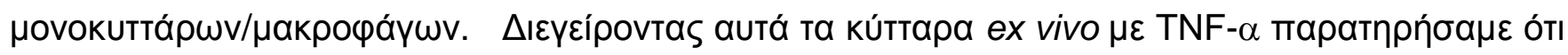

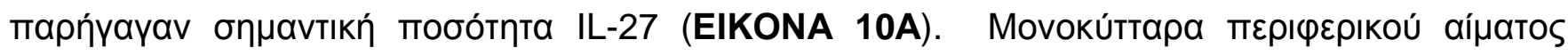

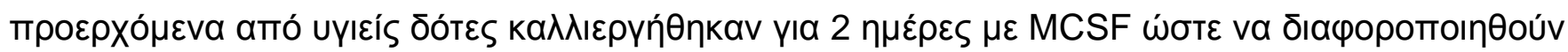

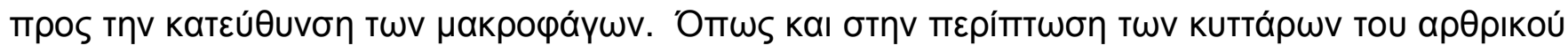

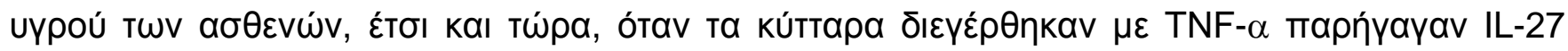

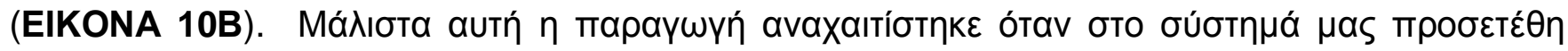

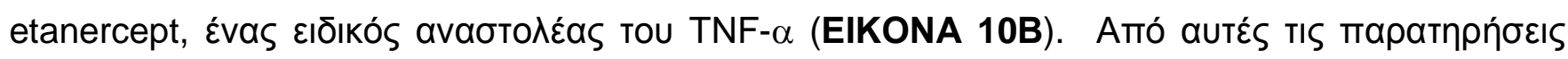

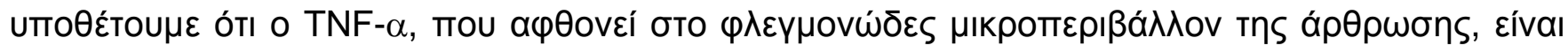




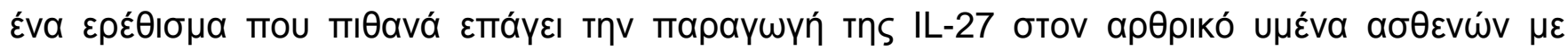

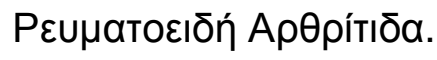




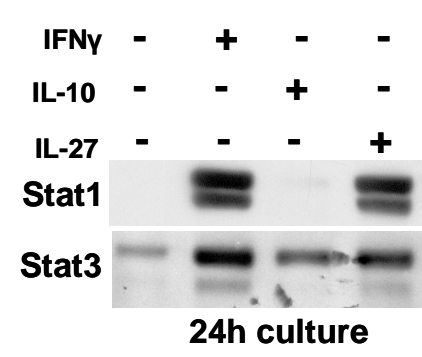

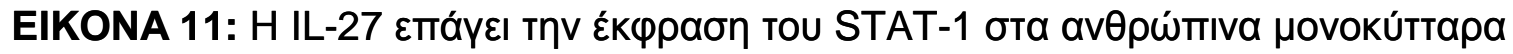

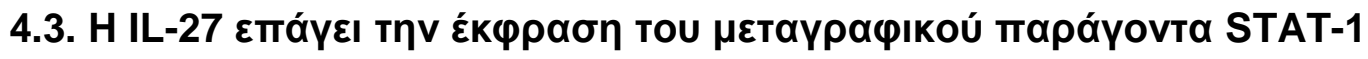

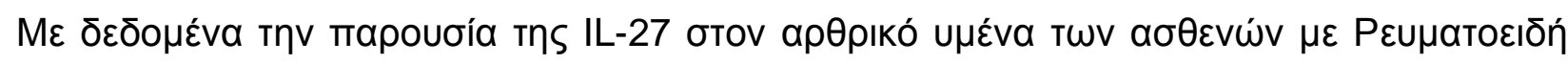

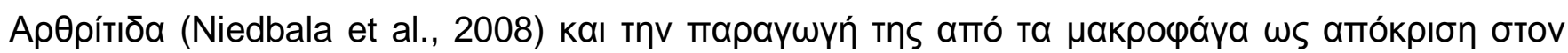

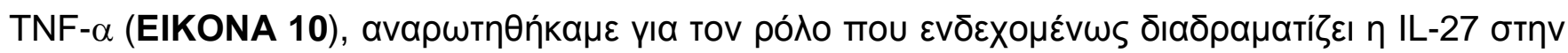

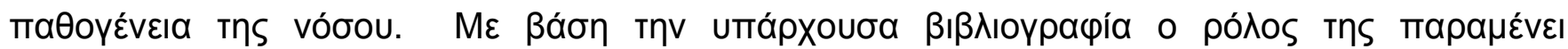

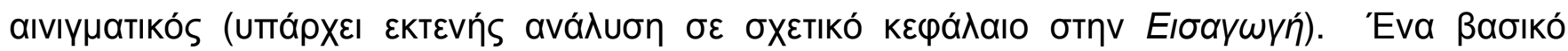

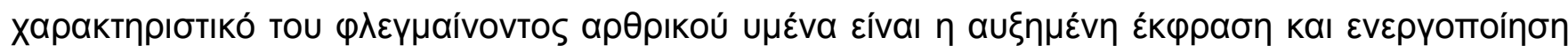

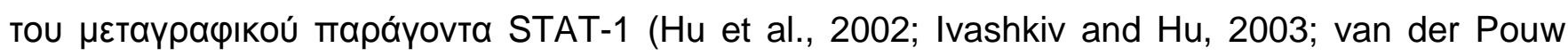

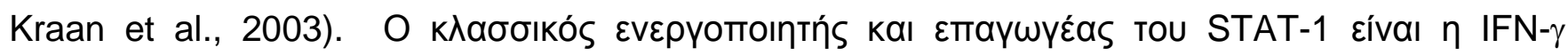

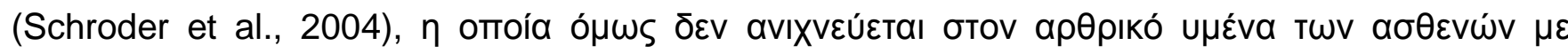

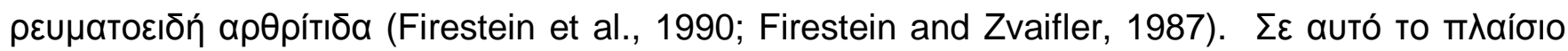

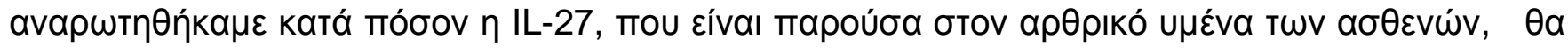

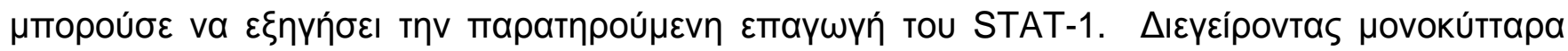

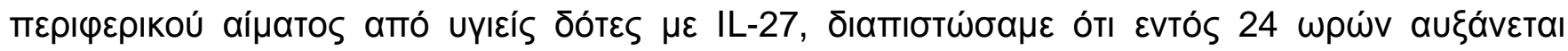

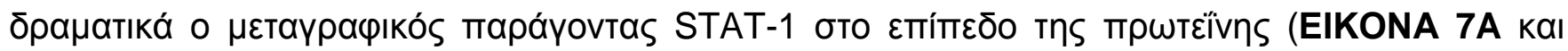

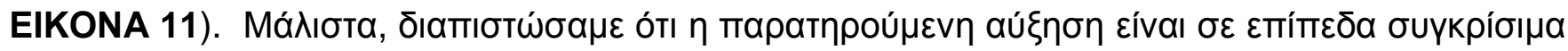

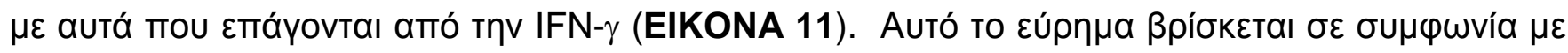

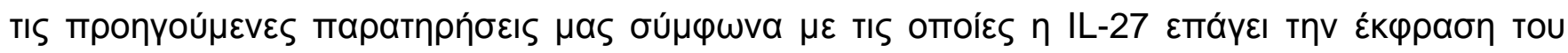




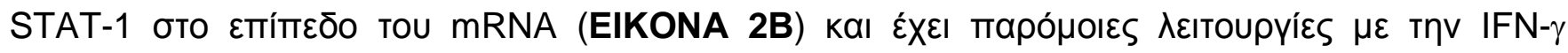
(EIKONA 2A). 


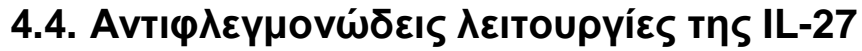

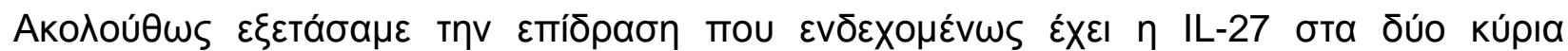

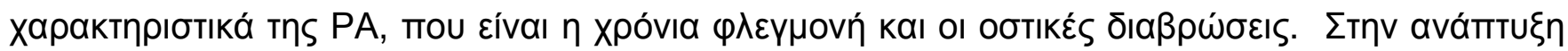

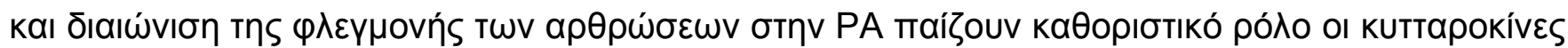
IL-1 $\beta$ kal TNF- $\alpha$ (Burger et al., 2006; Dayer, 2003; Feldmann et al., 2001; Feldmann and Maini,

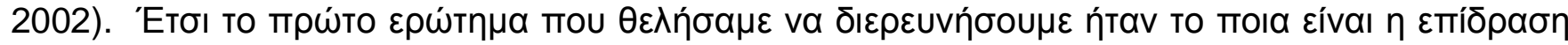

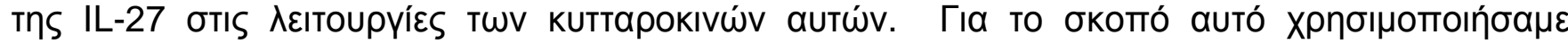

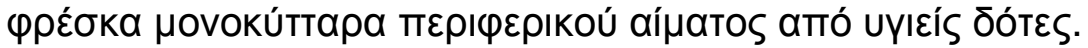

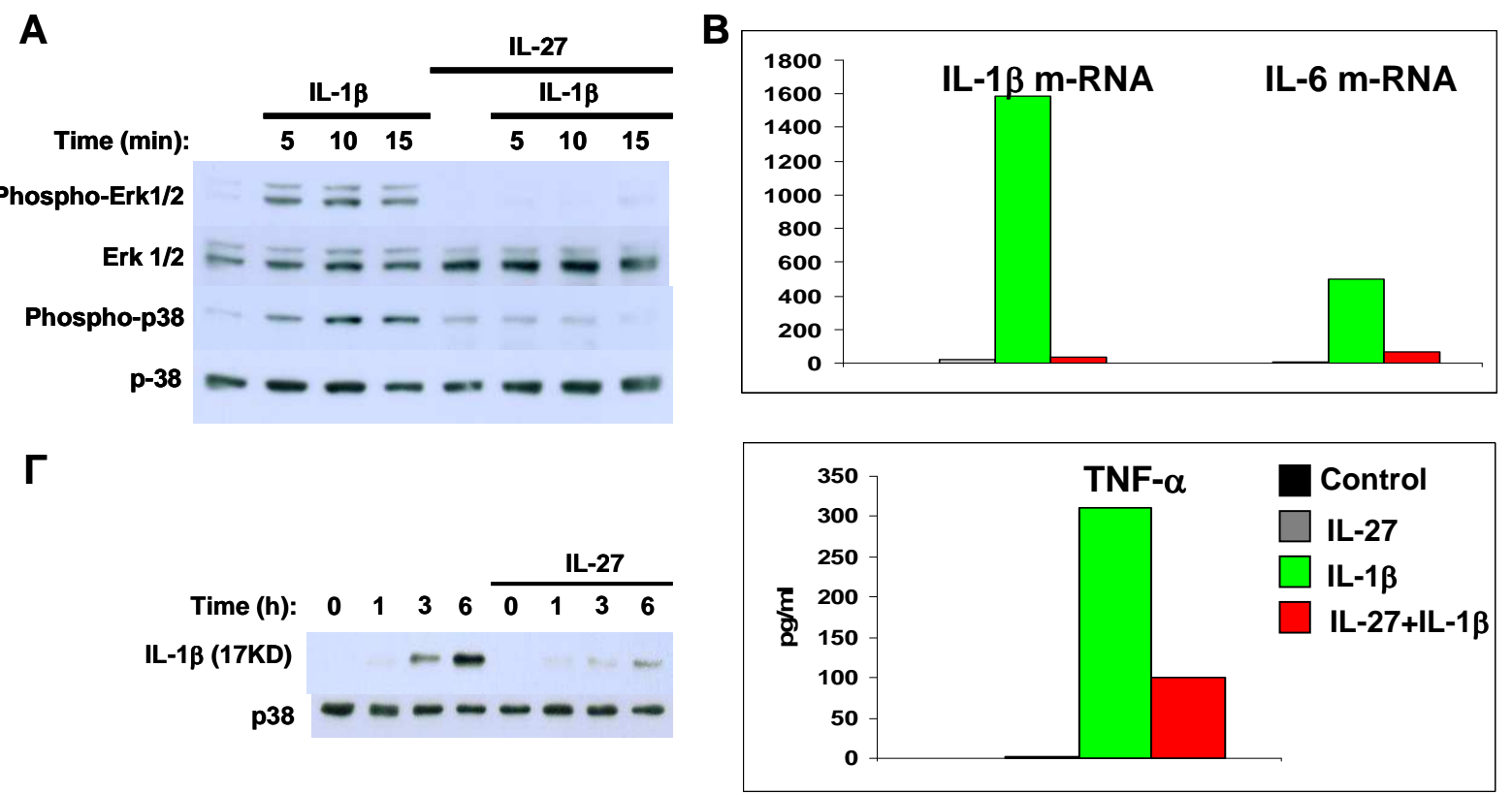

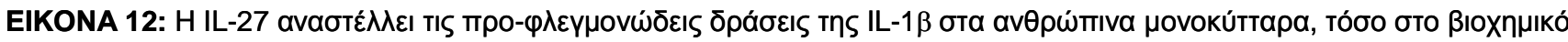

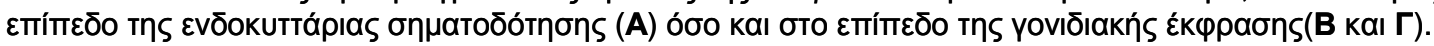

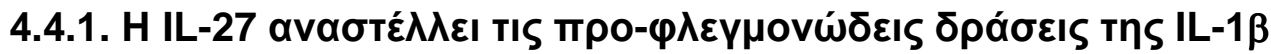

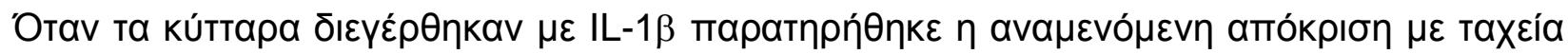

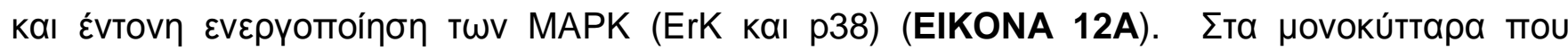

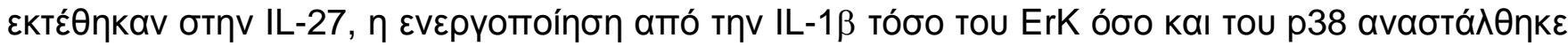




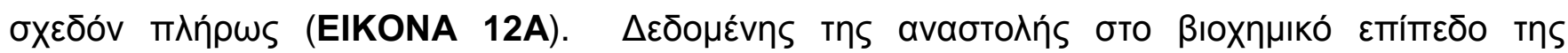

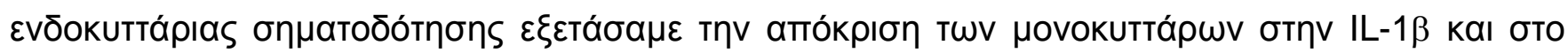

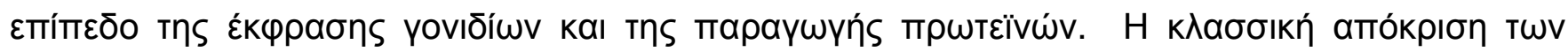

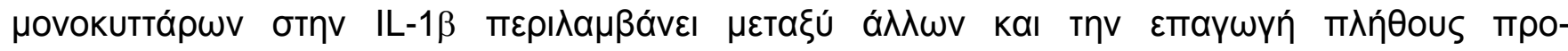

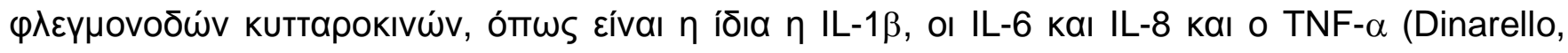

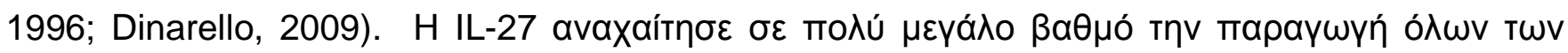

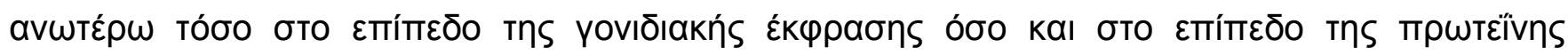
(EIKONA 12B каı Г).

A

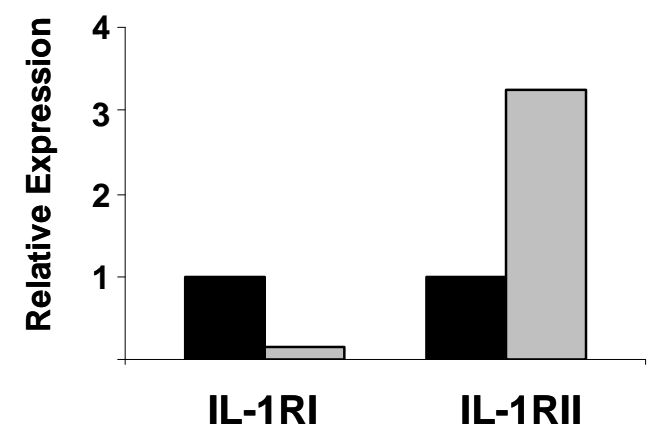

B

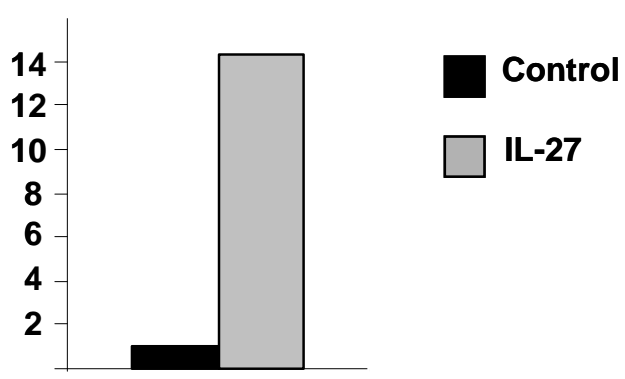

IL-1Ra

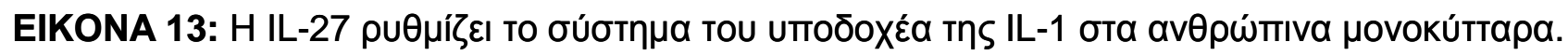

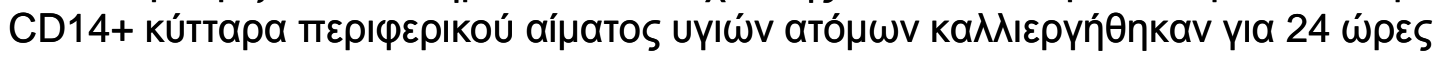

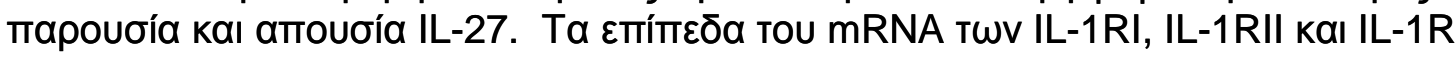

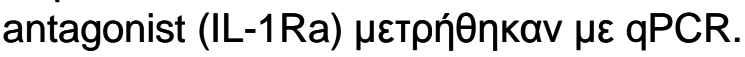

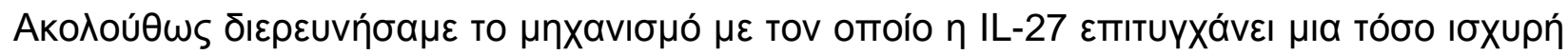

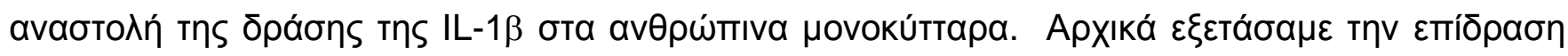

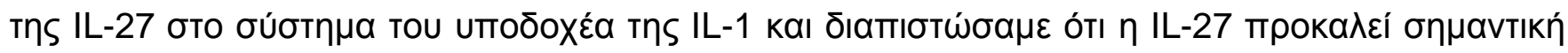

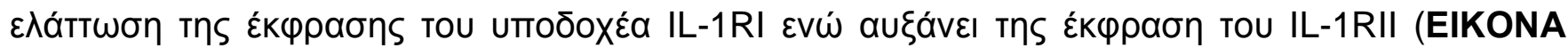

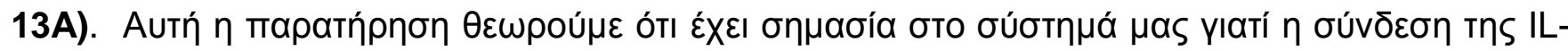




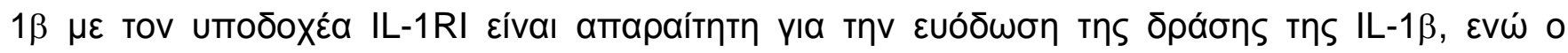

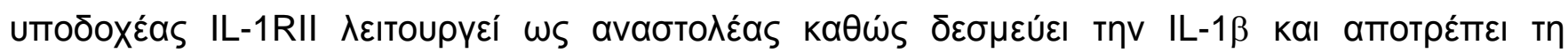

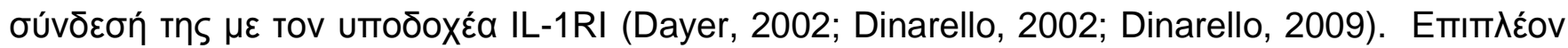

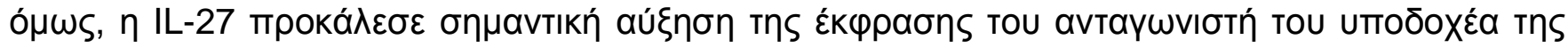

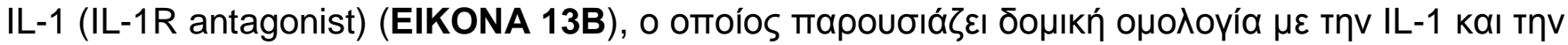

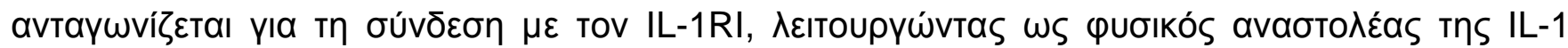

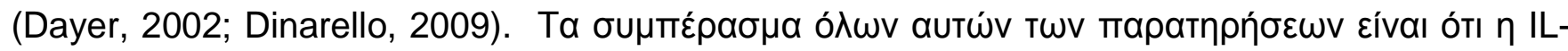

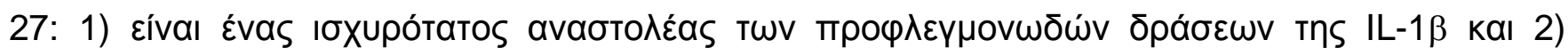

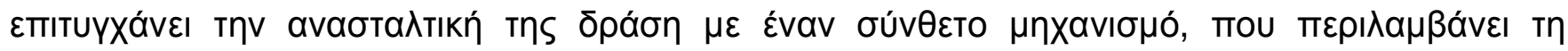

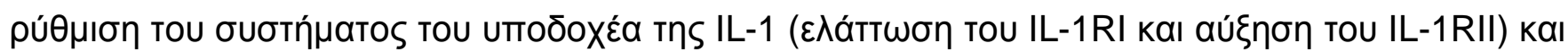

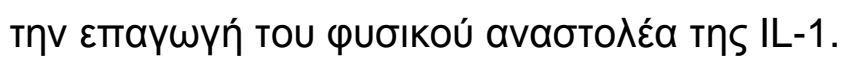


A

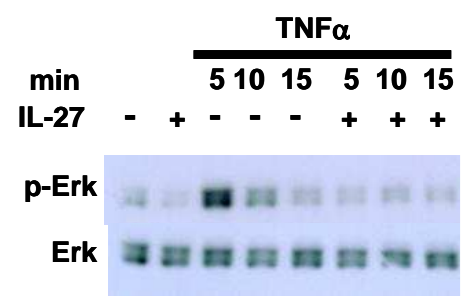

B

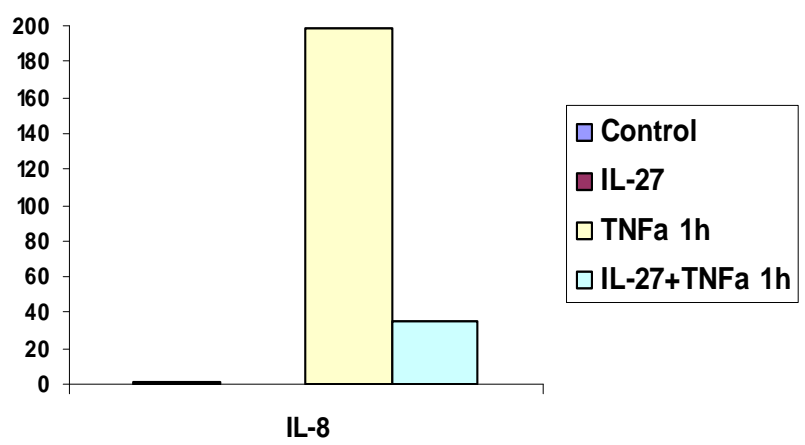

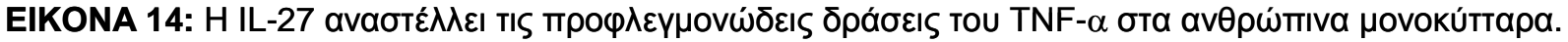

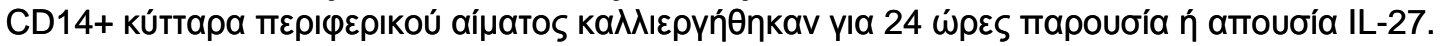

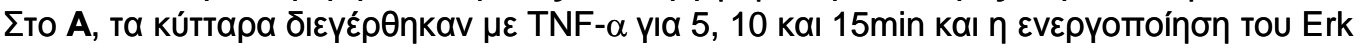

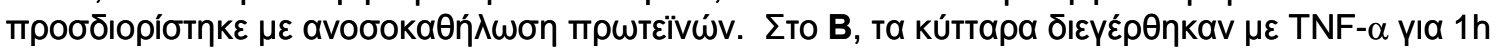

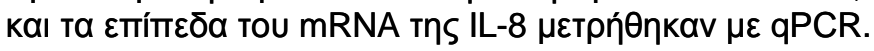

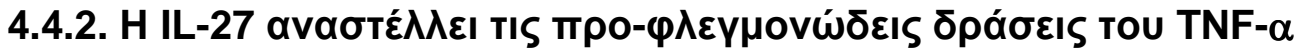

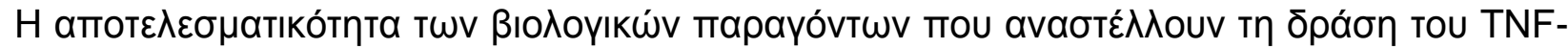

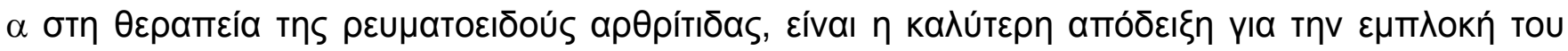

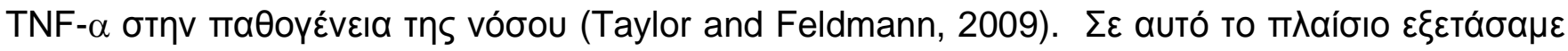

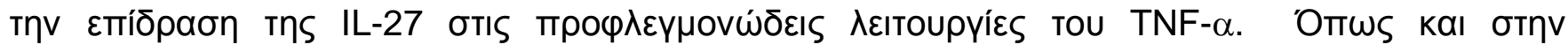

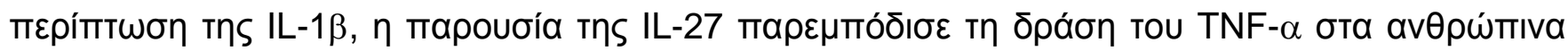

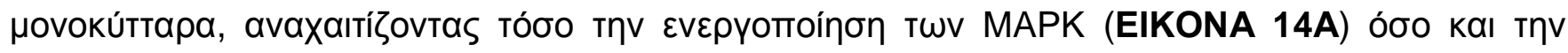

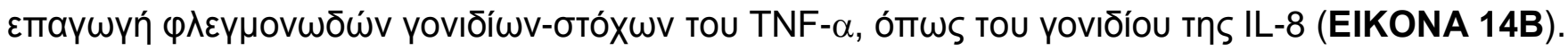



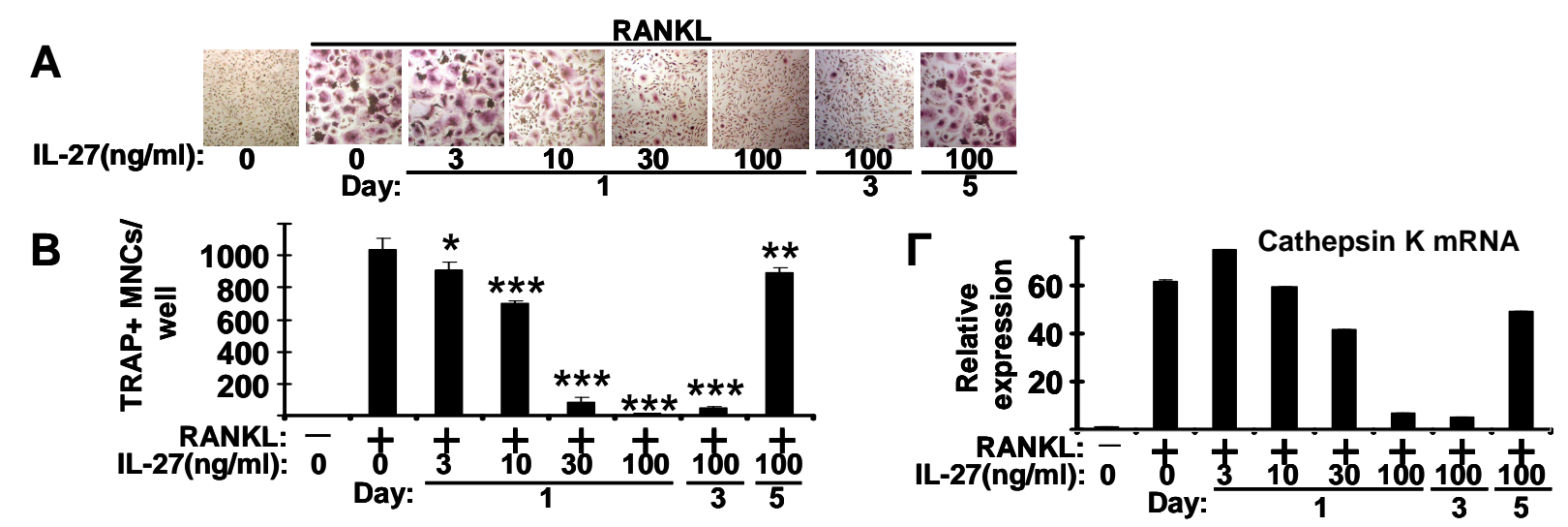

$\Delta$
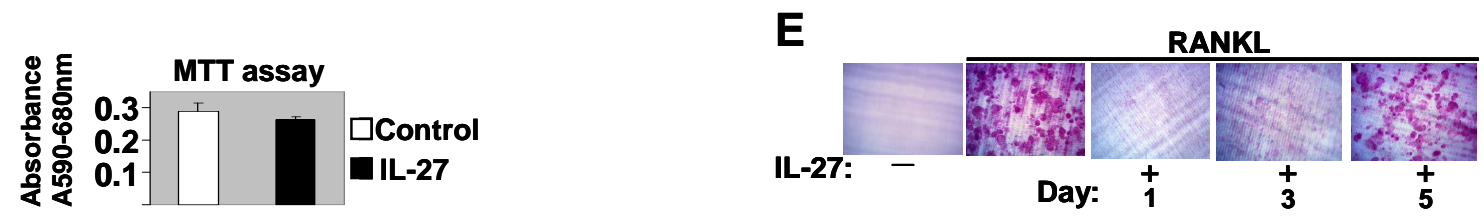

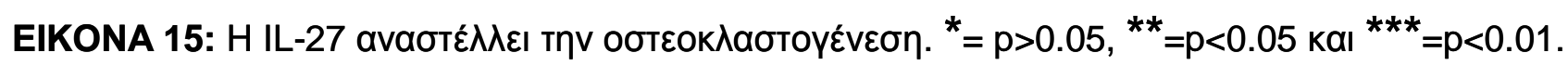

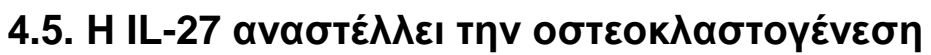

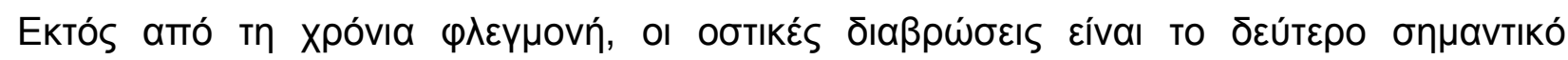

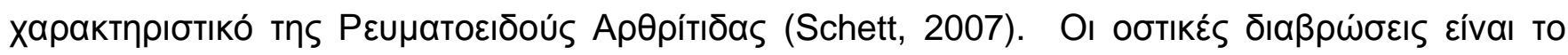

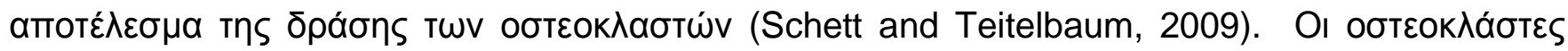

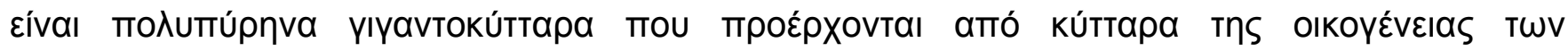

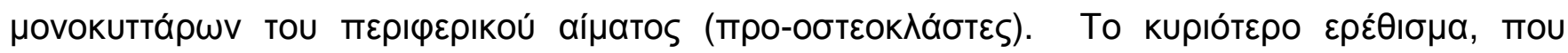

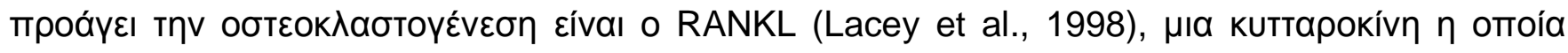

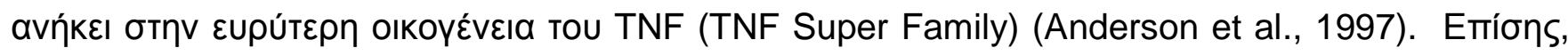

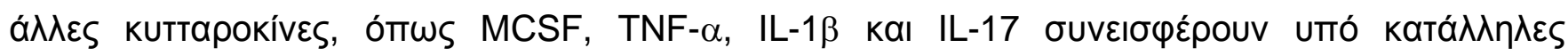

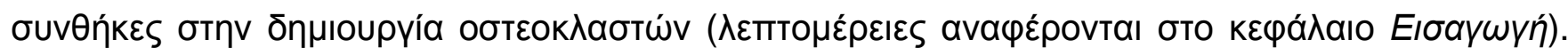

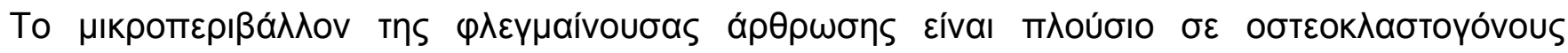

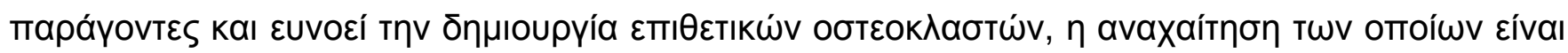

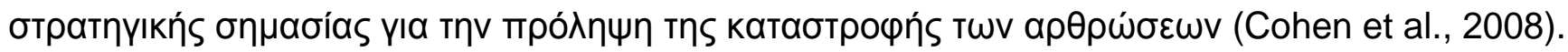




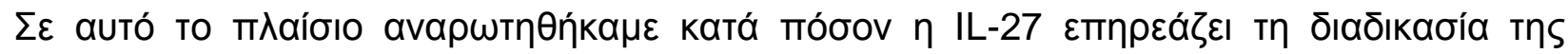

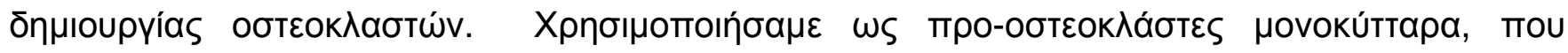

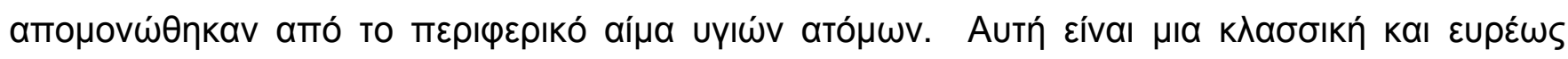

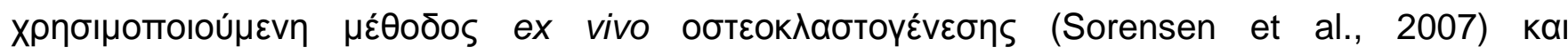

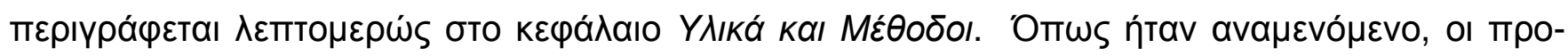

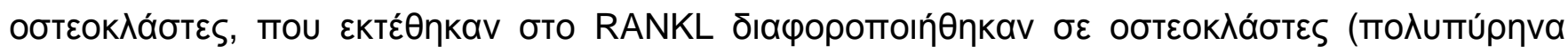

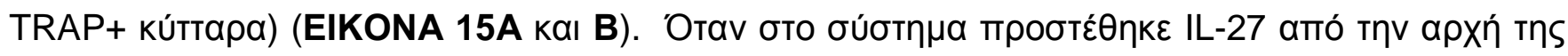

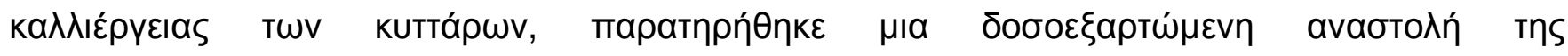

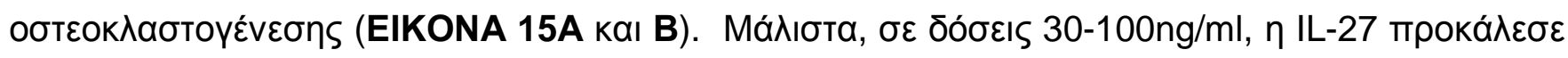

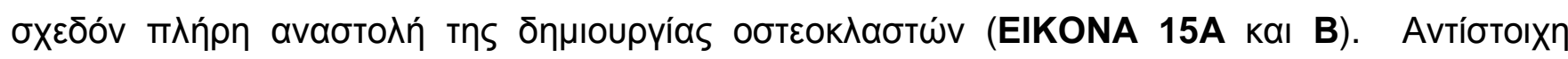

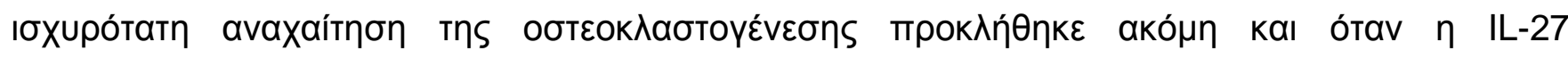

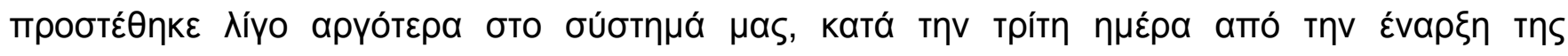

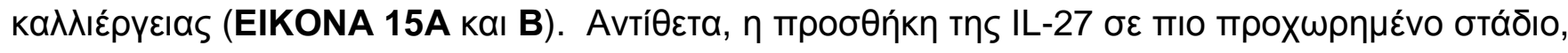

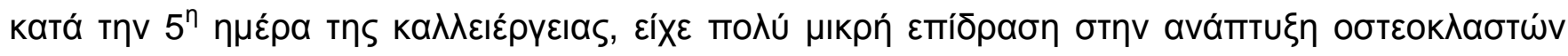

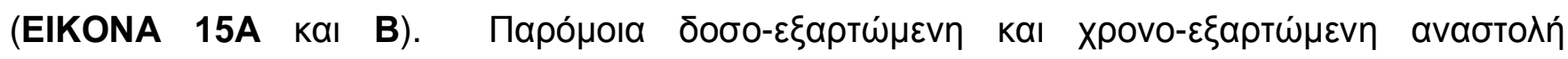

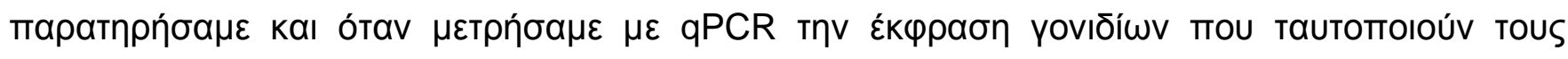

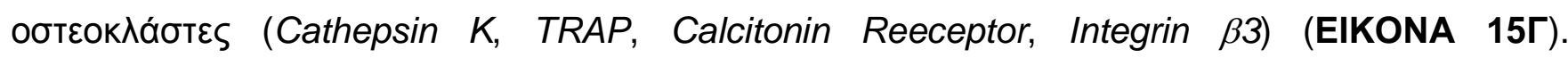

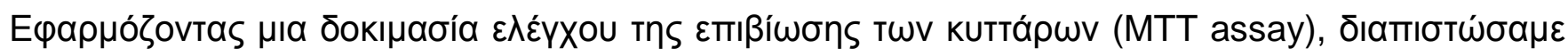

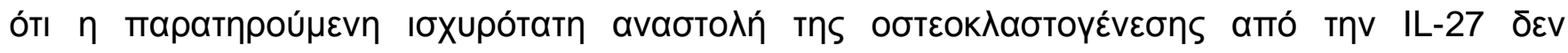

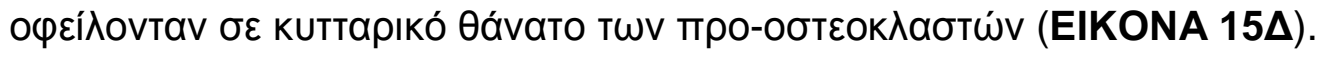

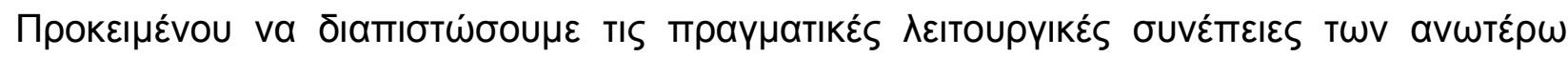

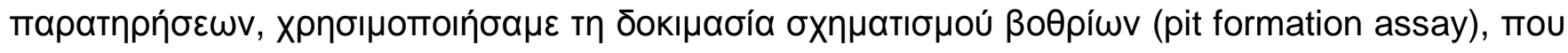

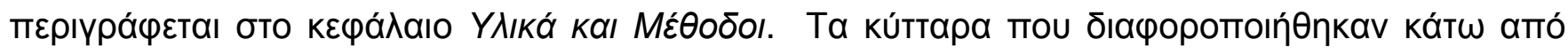




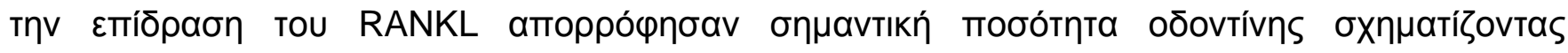

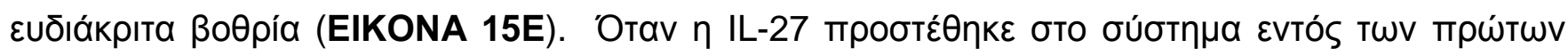

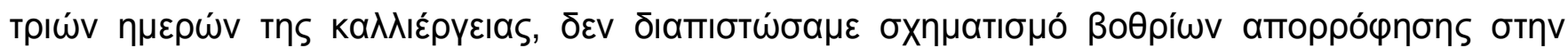

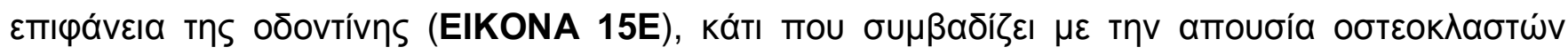

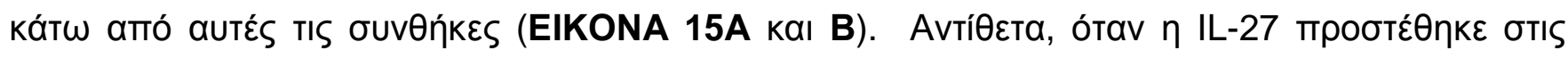

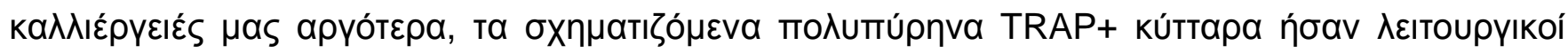

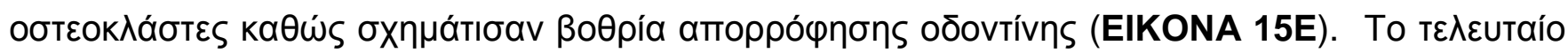

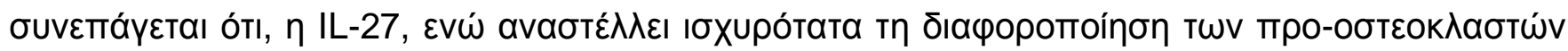

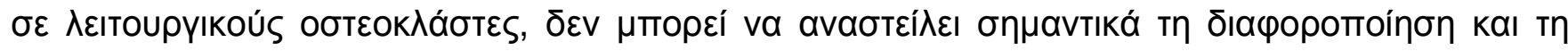

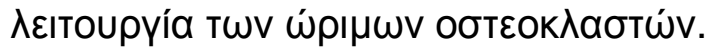



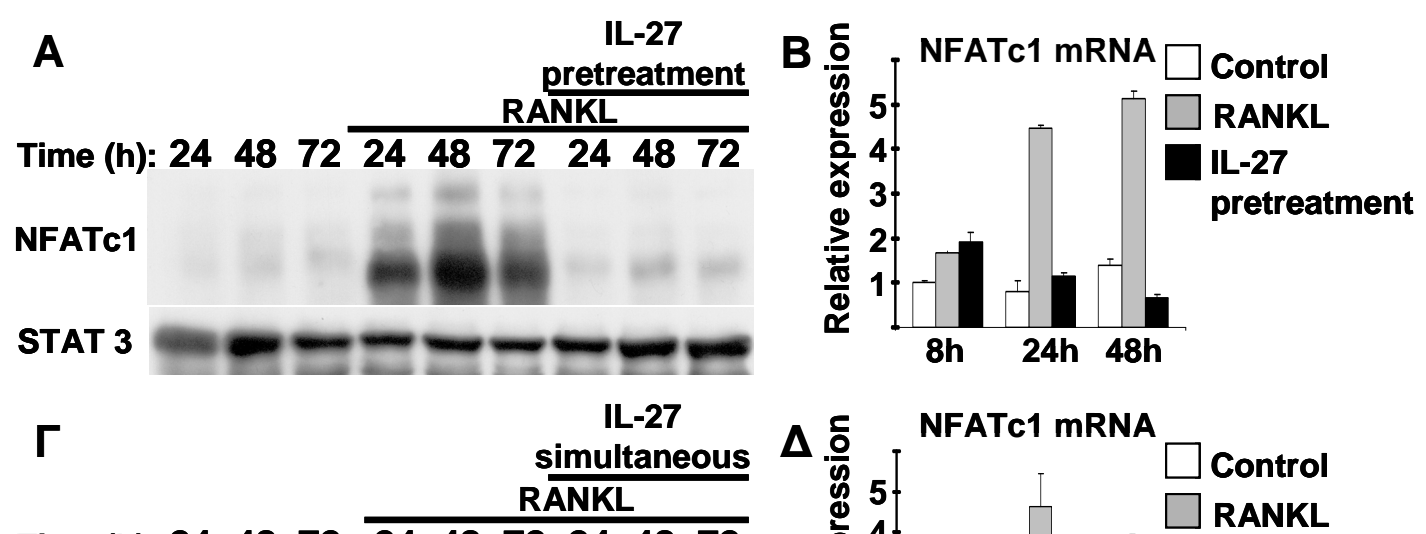

Time (h): $2 4 4 8 7 2 \longdiv { 2 4 4 8 7 2 \quad 2 4 4 8 7 2 }$ NFATc1

STAT 3

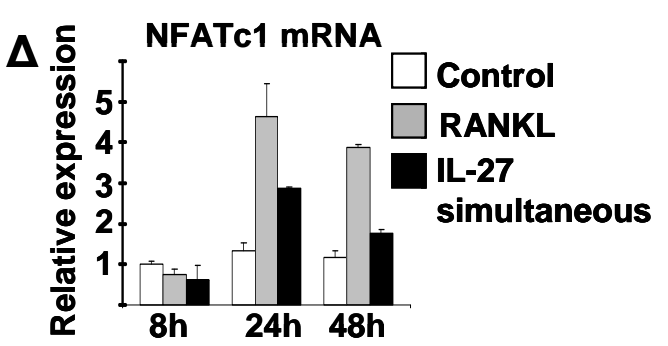

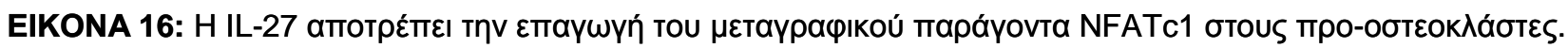

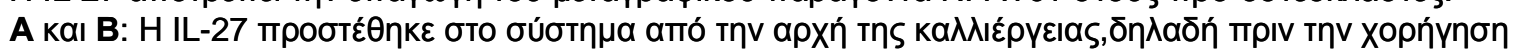

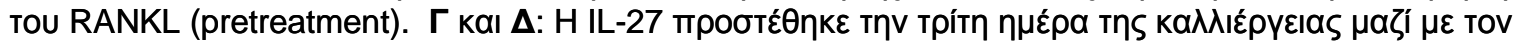
RANKL (simultaneous).

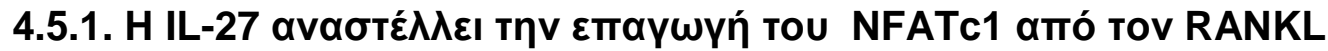

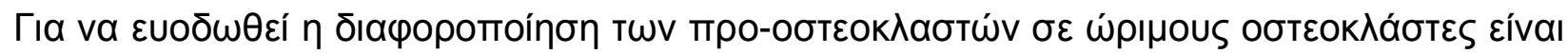

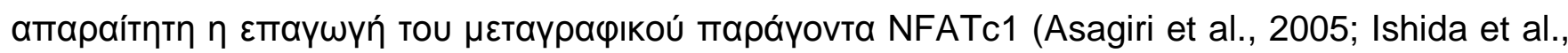
2002; Koga et al., 2005; Negishi-Koga and Takayanagi, 2009; Stern, 2006; Takayanagi, 2007b;

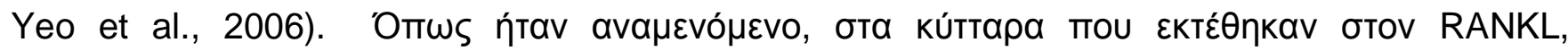

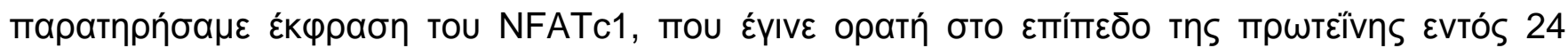

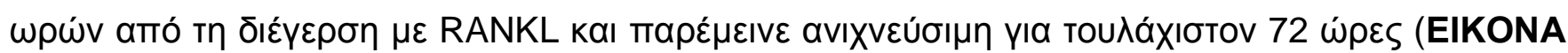

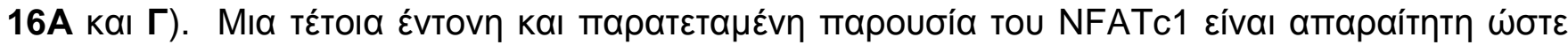

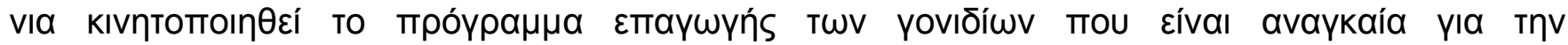

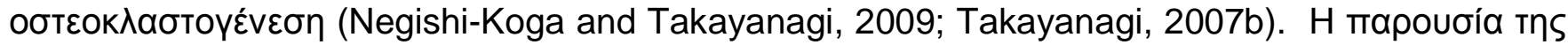

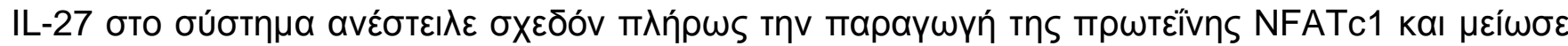

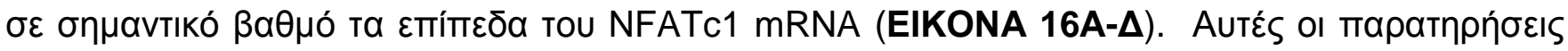




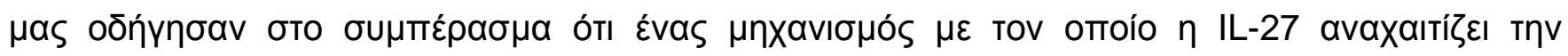

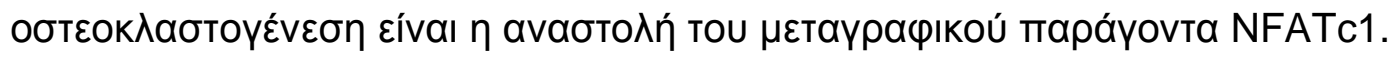



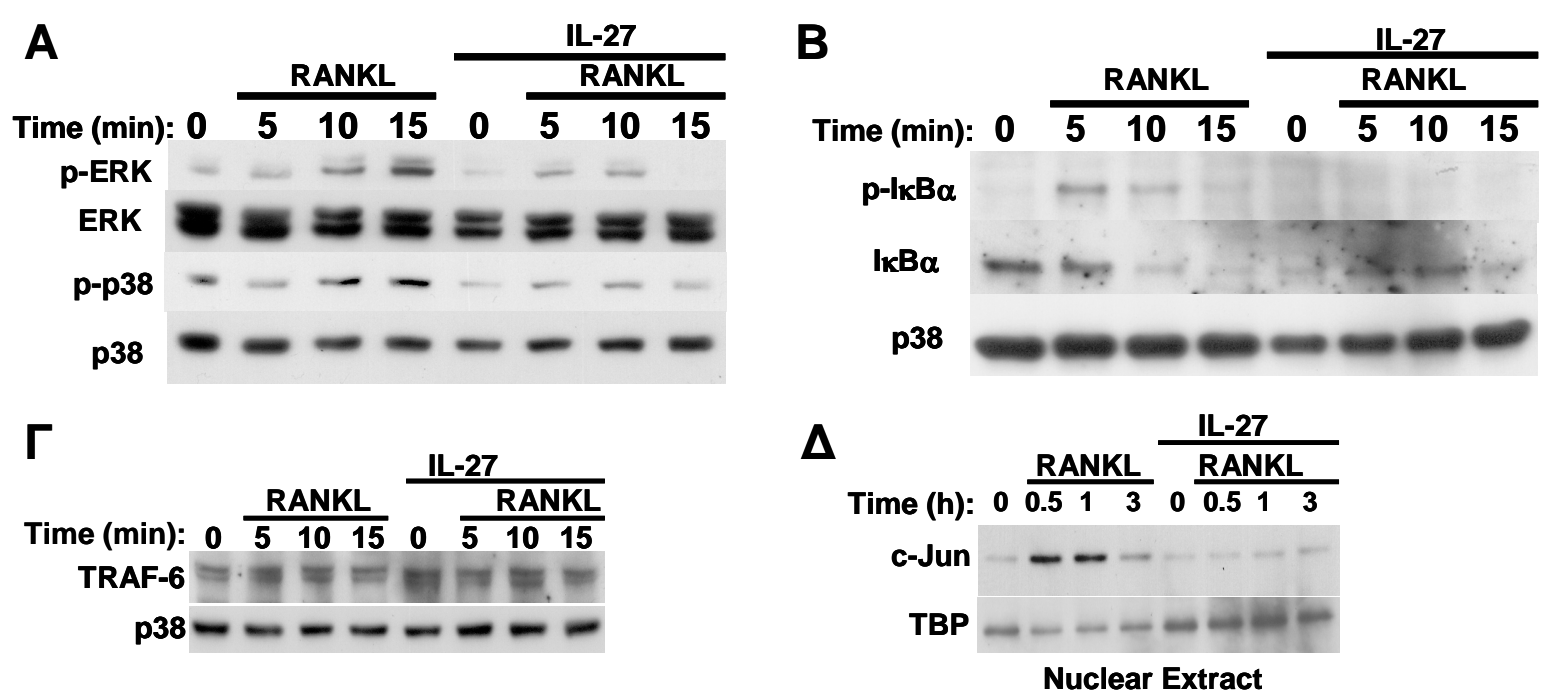

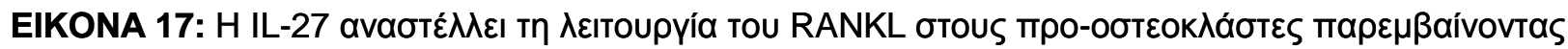

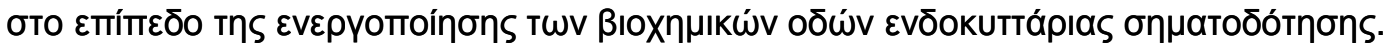

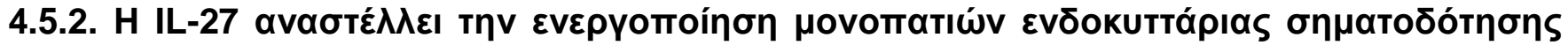

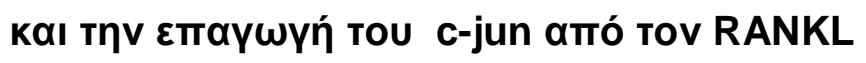

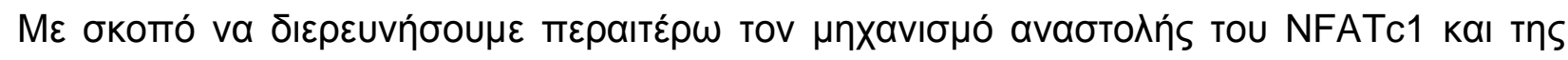

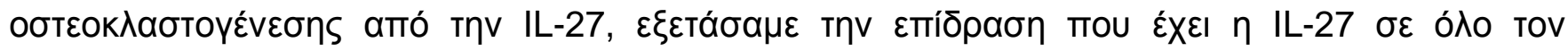

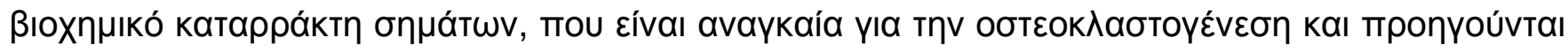

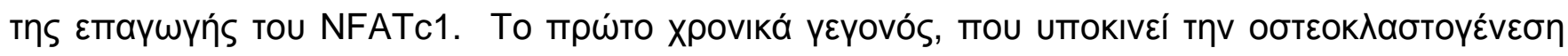

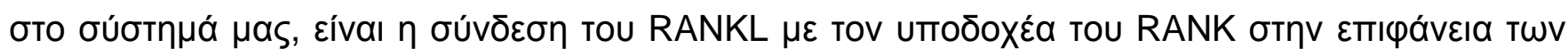

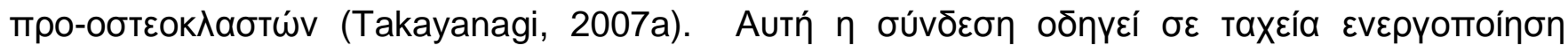

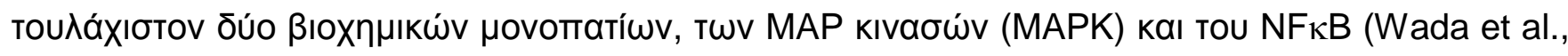

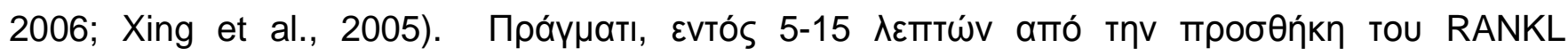

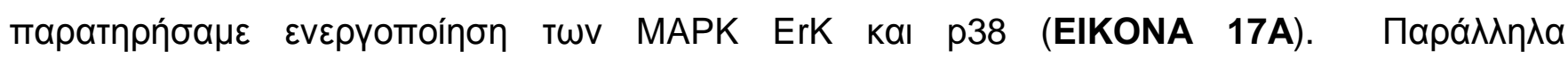

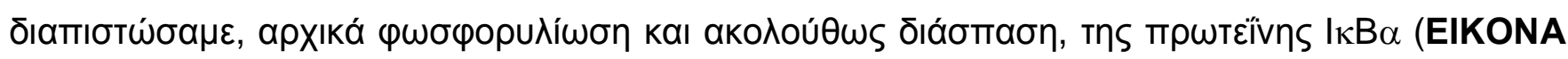

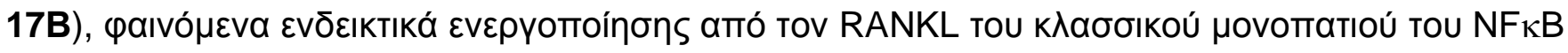


(Brown et al., 2008; Chiu et al., 2009; Vallabhapurapu and Karin, 2009). H парouбía tns IL-27

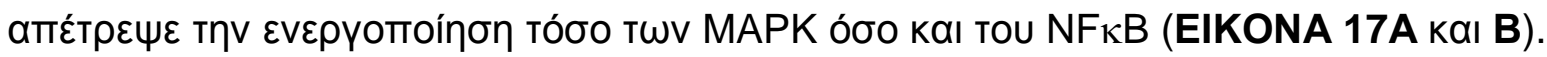

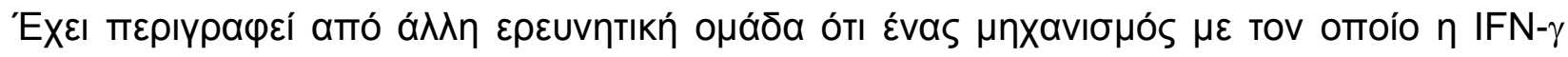

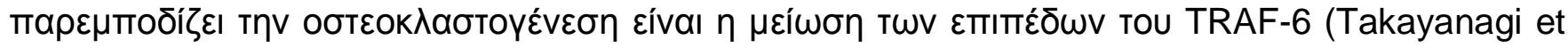

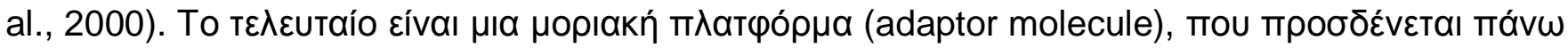

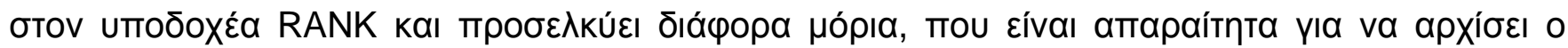

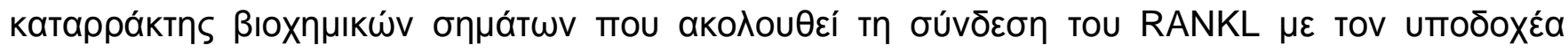

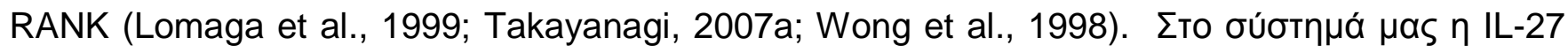

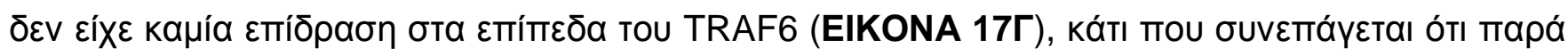

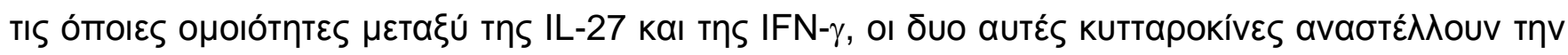

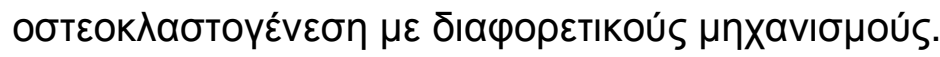

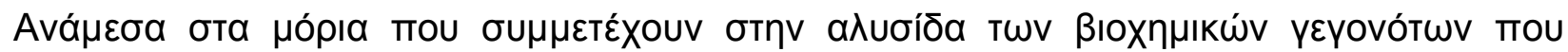

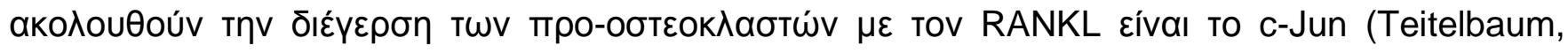

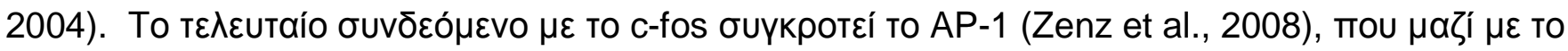

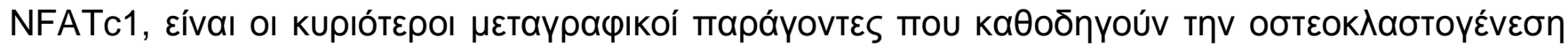

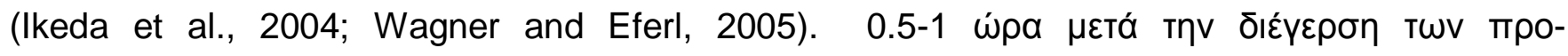

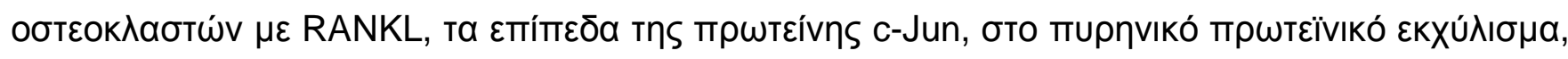

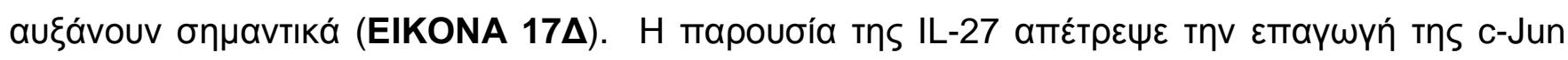

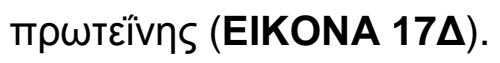




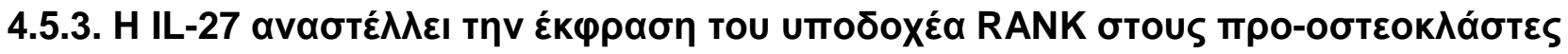

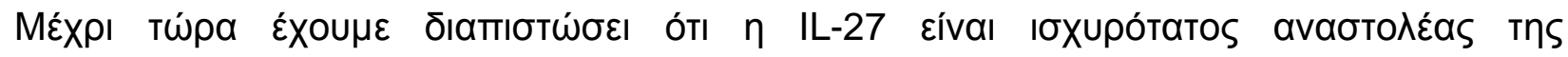

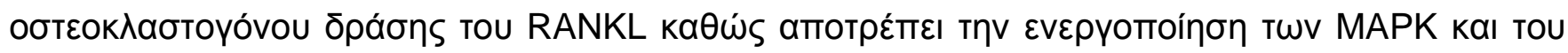

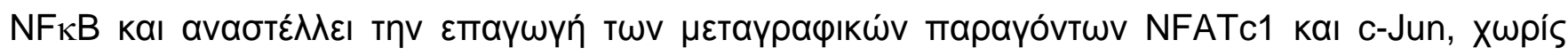

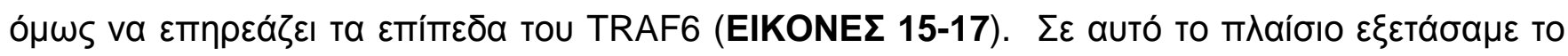

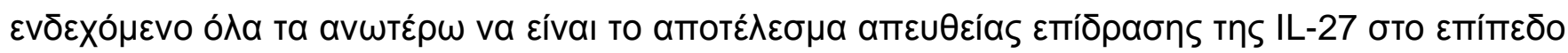

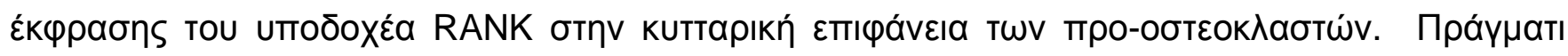

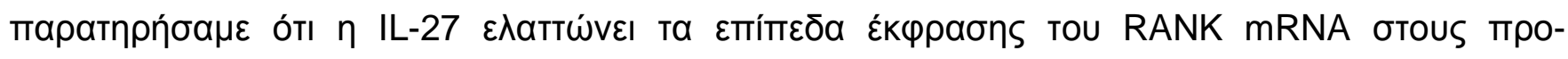

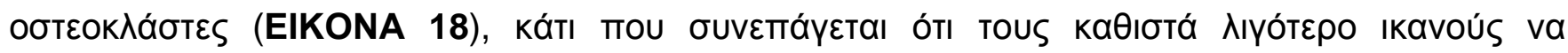

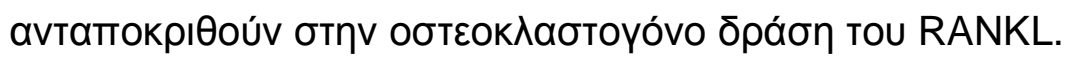

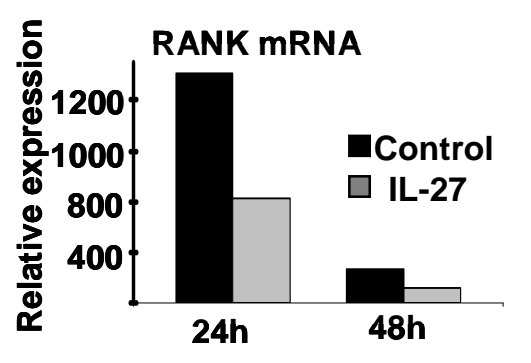

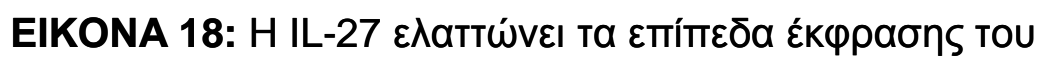

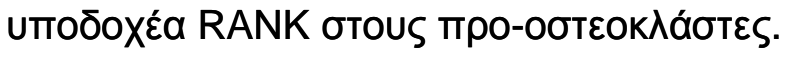

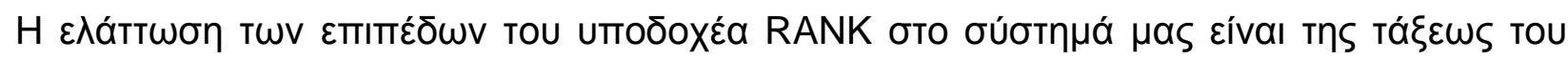

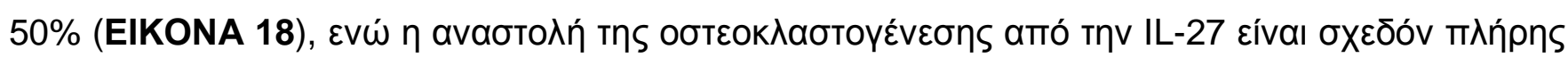

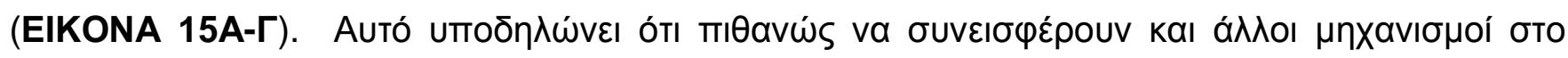

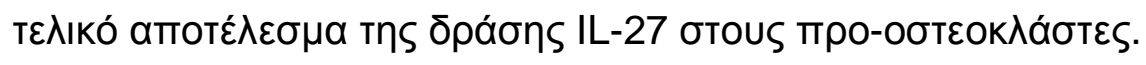



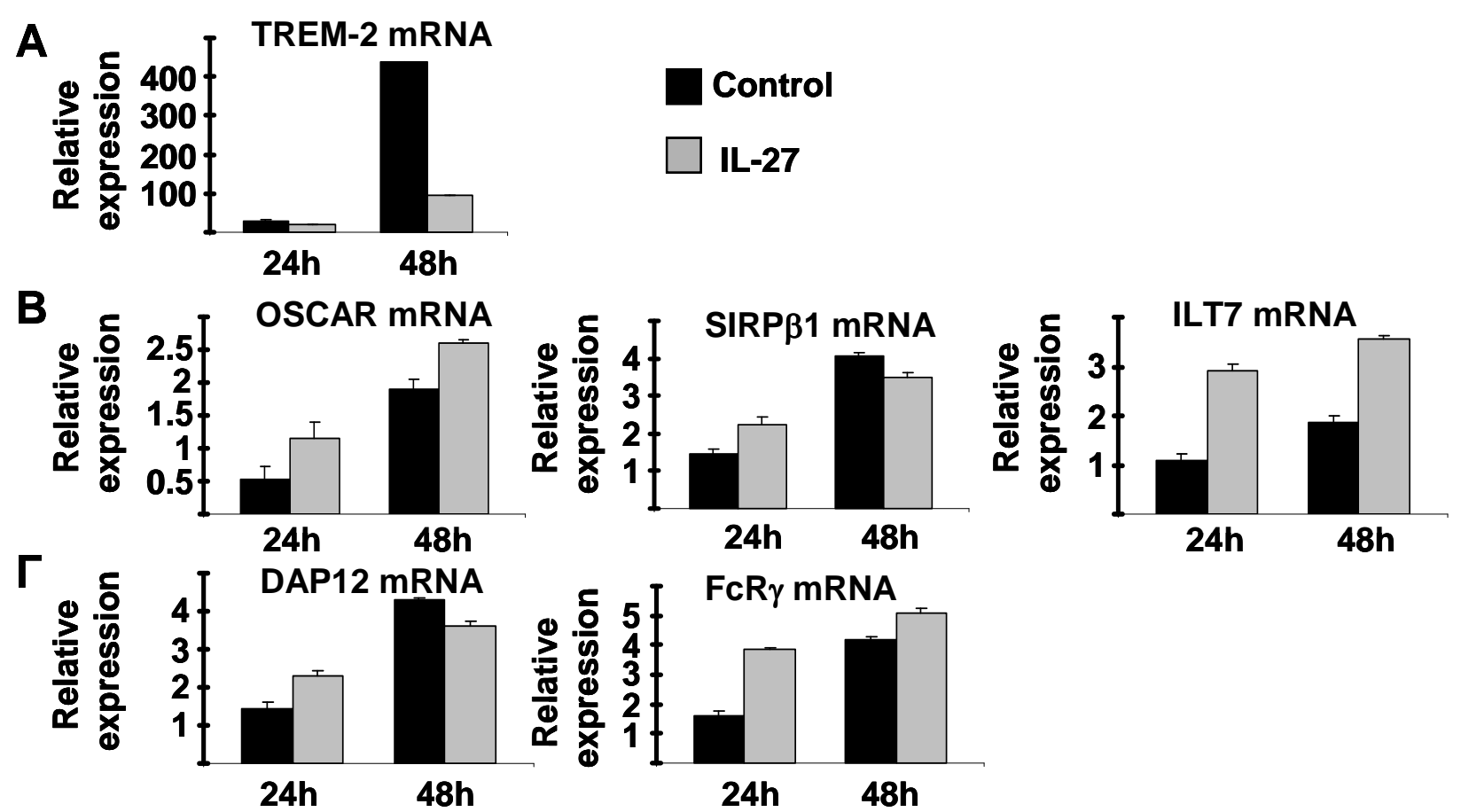

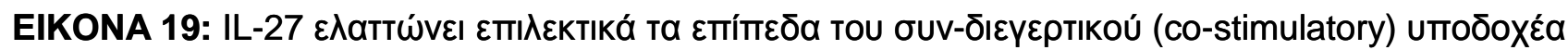

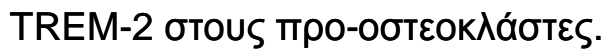

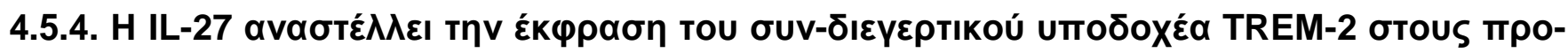

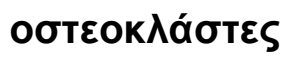

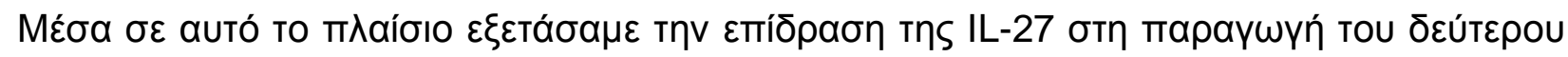

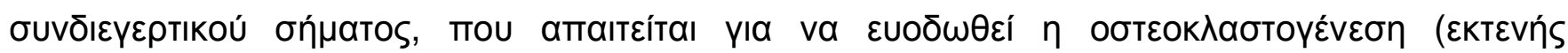

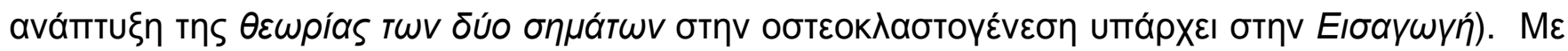

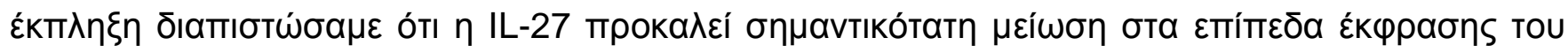

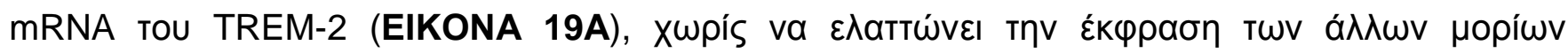

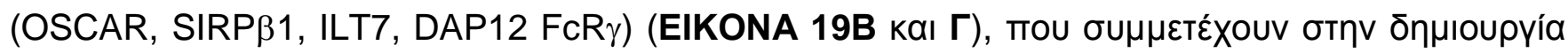

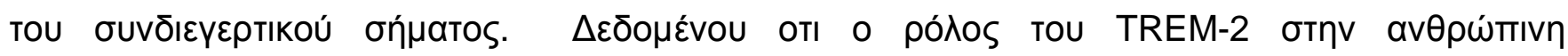

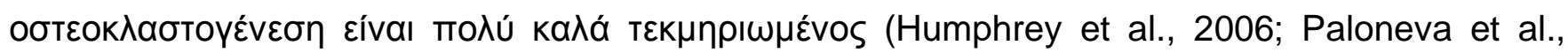

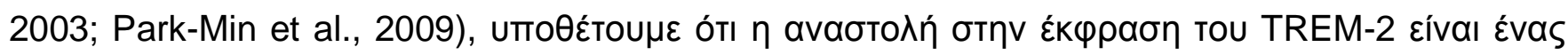




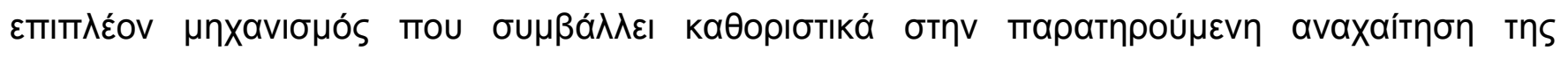

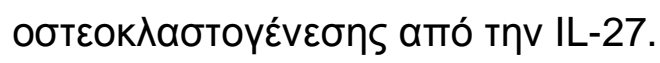


A

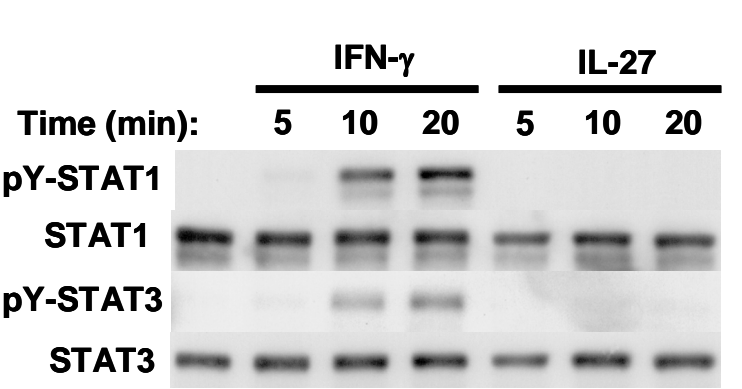

Movokútrapa

B

Cont IFN- $\gamma$ IL-6 IL-27

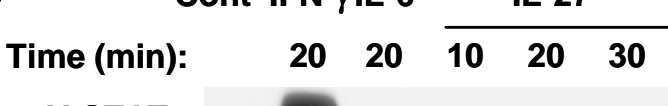
pY-STAT1

STAT1

pY-STAT3

STAT3

$\Delta$

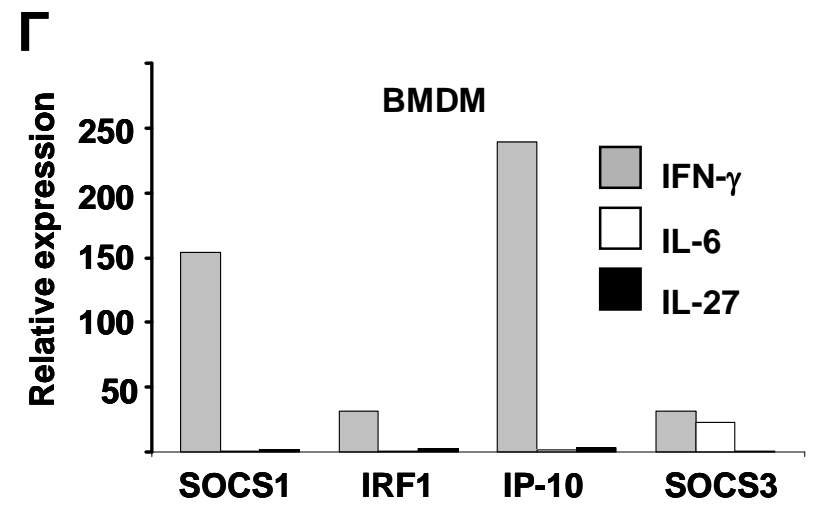

Makpọáya (BMDM)

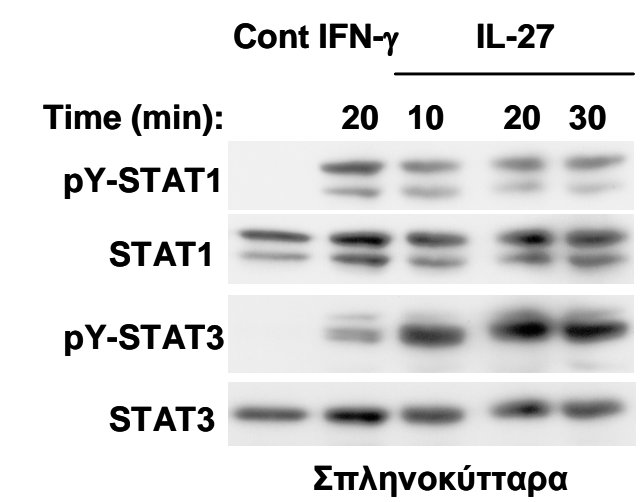

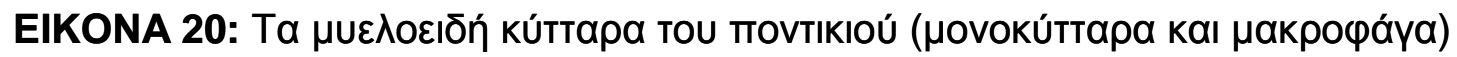

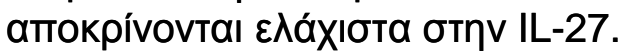

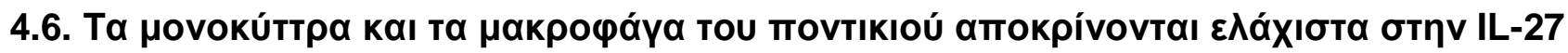

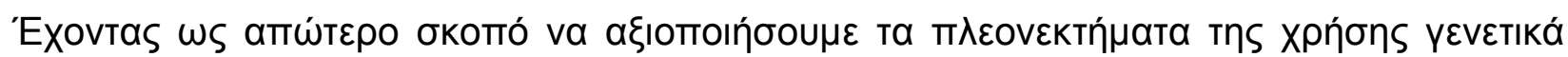

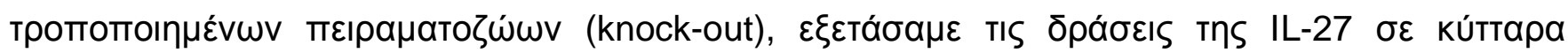

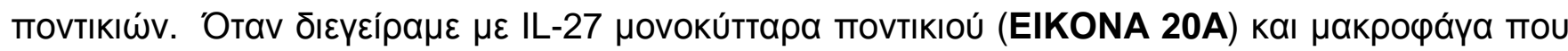

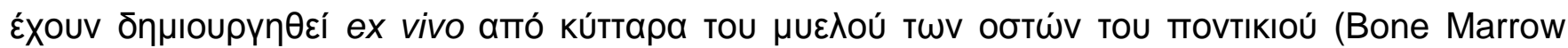

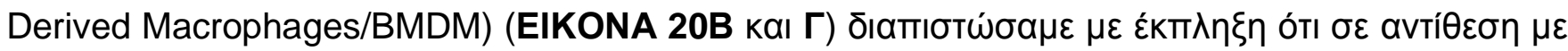

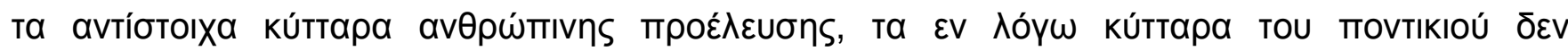

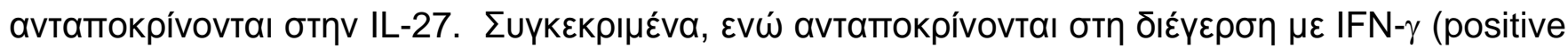

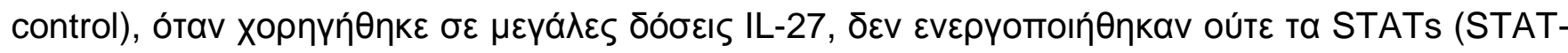

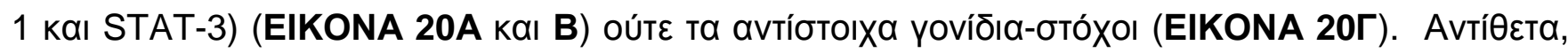




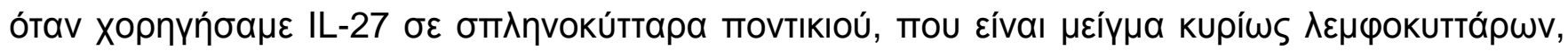

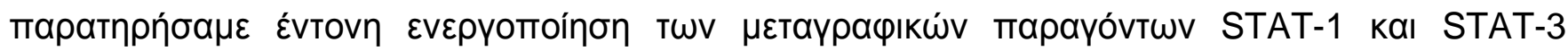

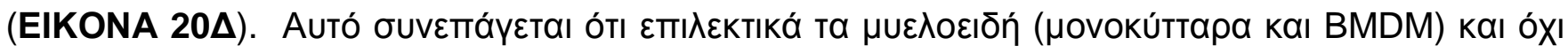

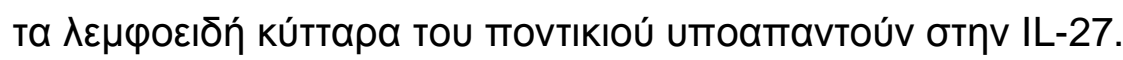

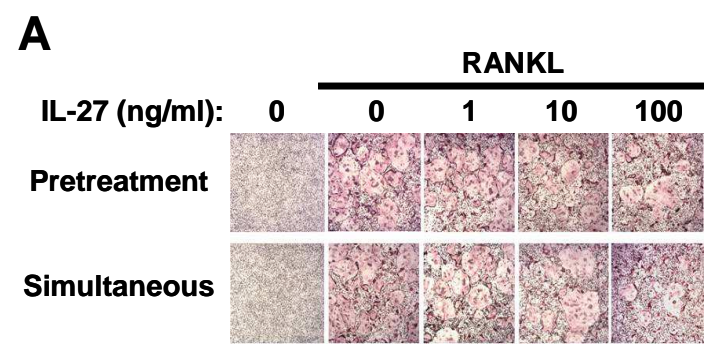

B

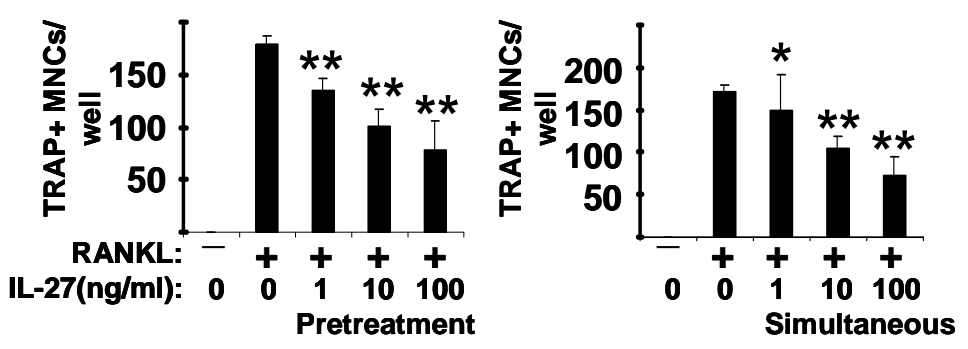

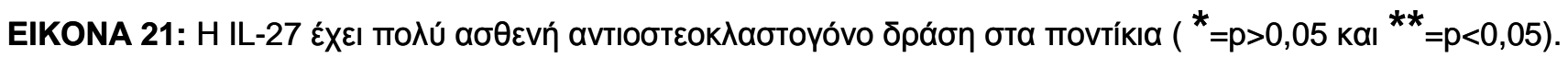

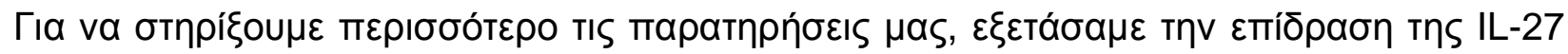

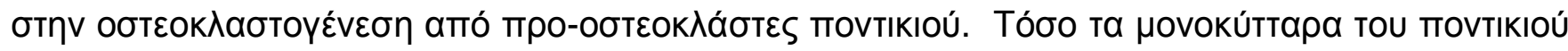

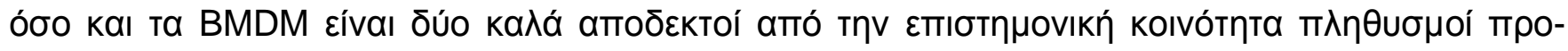

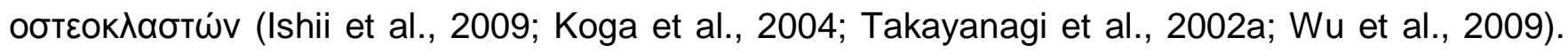

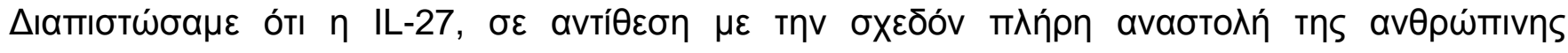

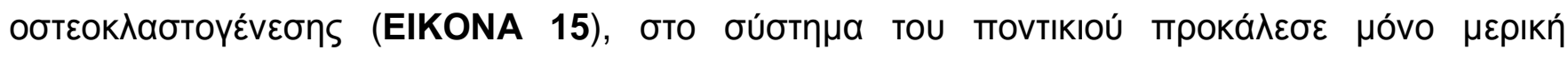

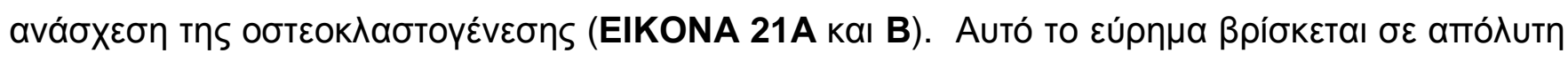

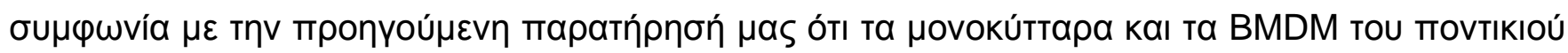

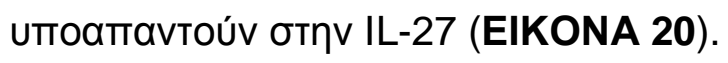




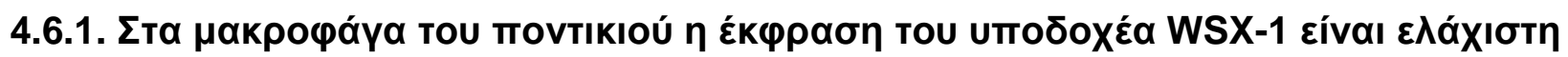

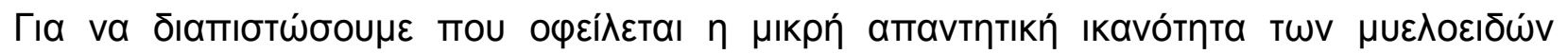

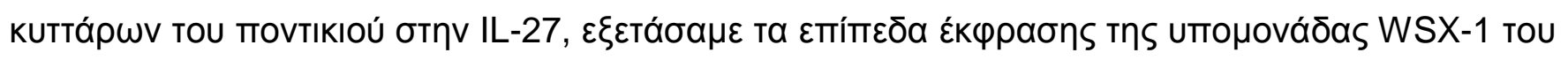

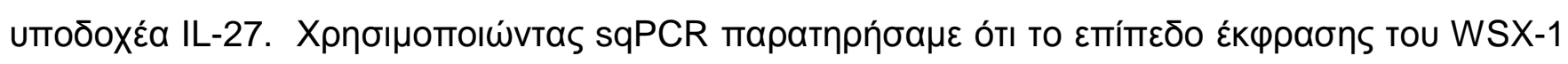

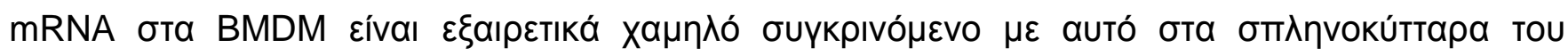

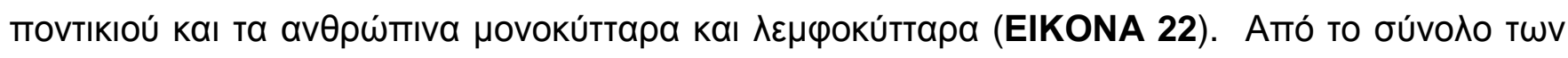

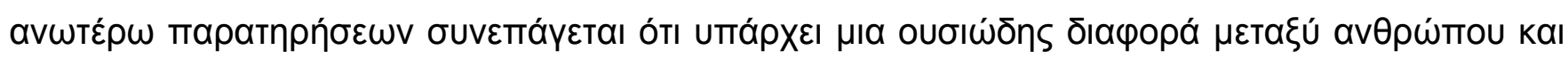

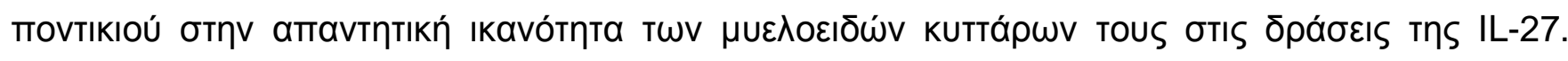

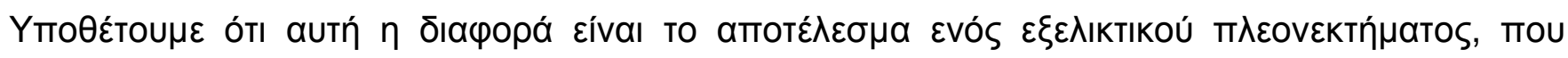

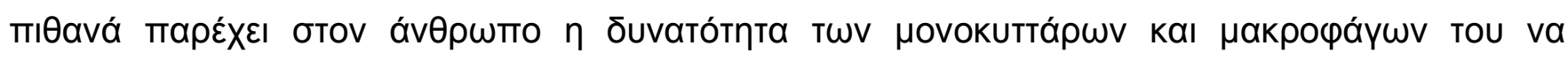

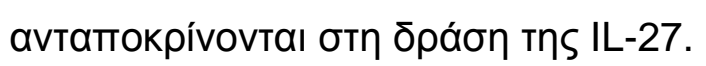

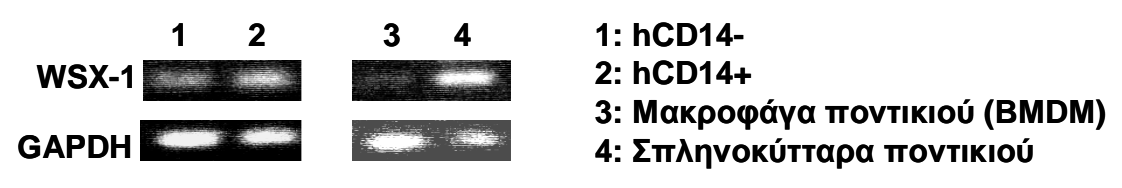

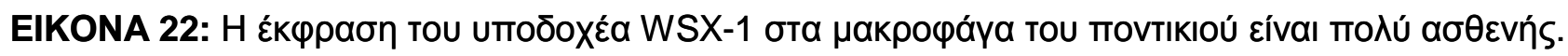




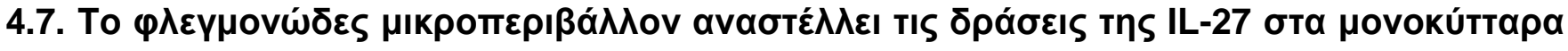 каı та накроча́үa}

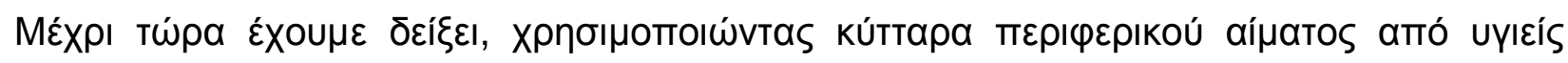

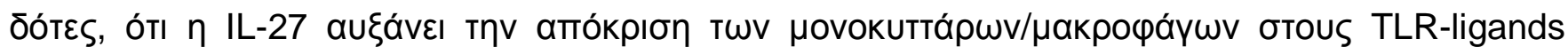

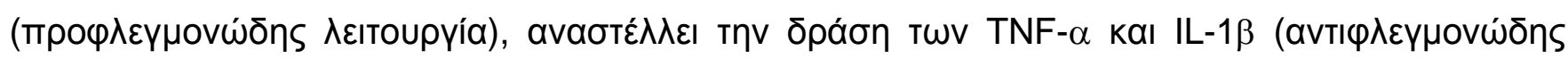

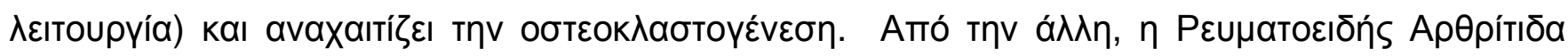

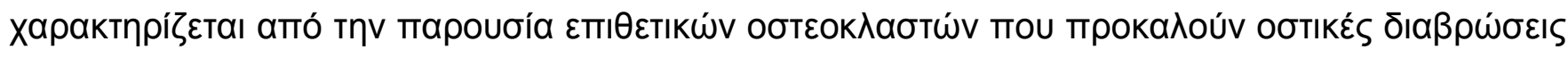

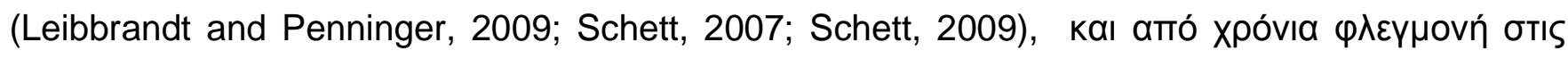

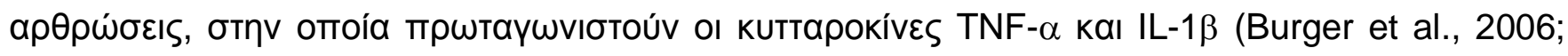
Dayer, 2003; Feldmann, 2008; Feldmann et al., 2001; Feldmann and Maini, 2002). $\Sigma \varepsilon$ auтó то

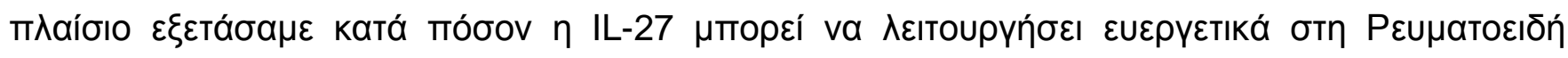

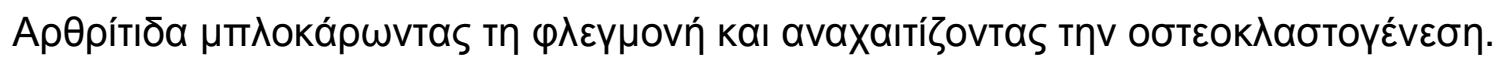




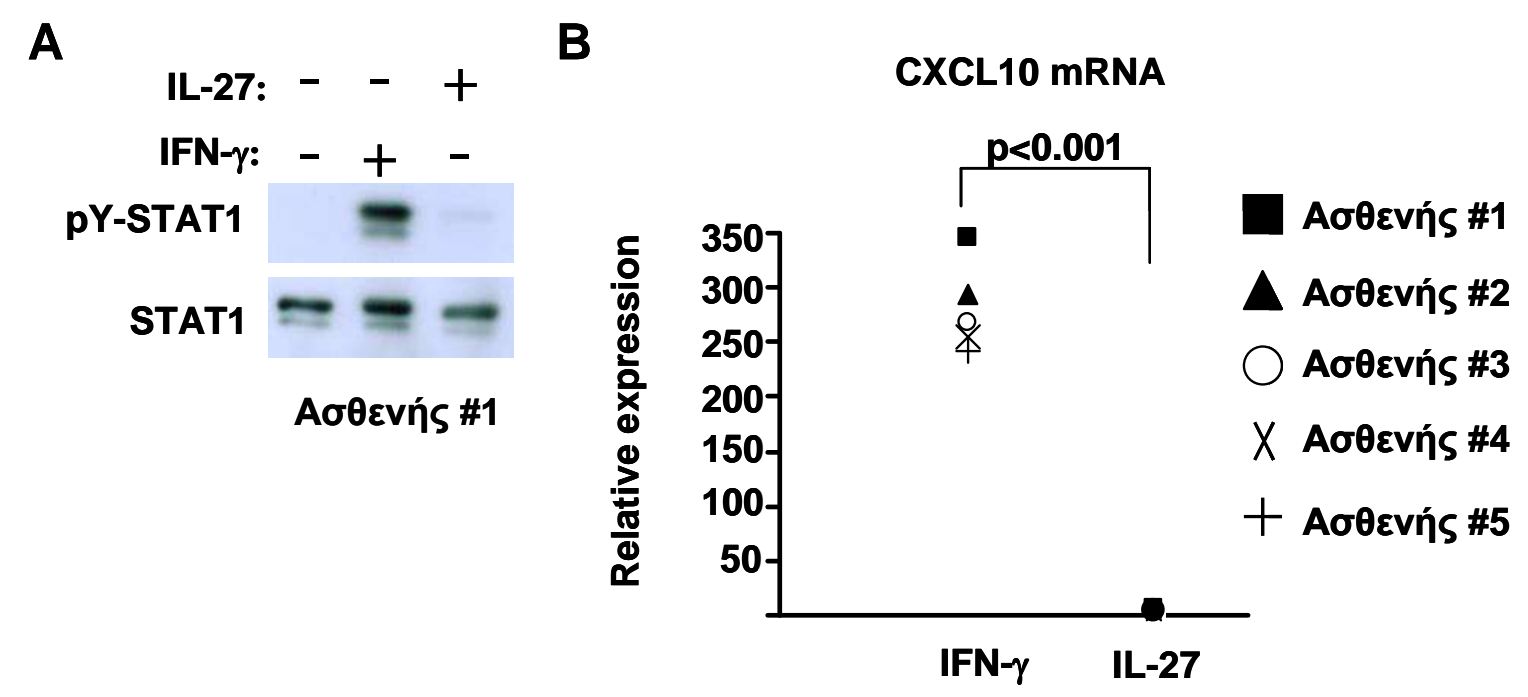

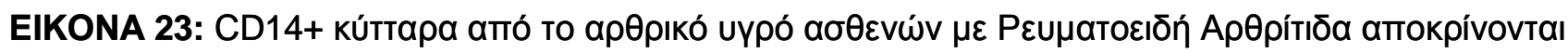

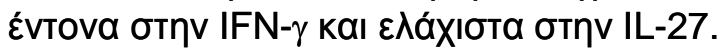

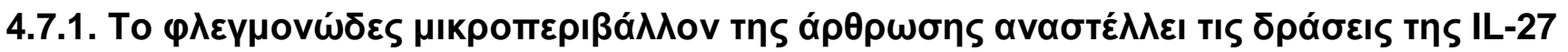

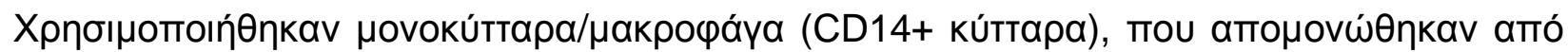

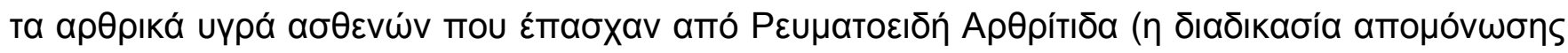

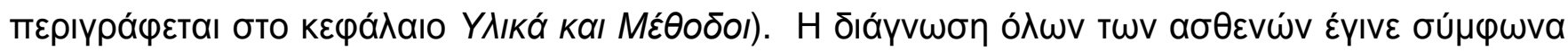

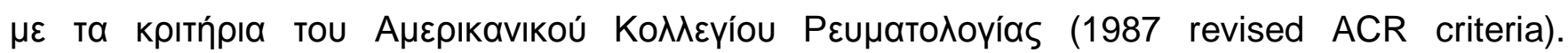

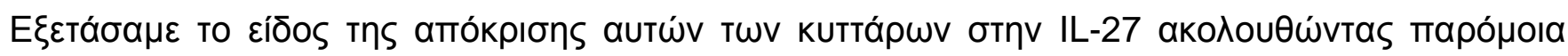

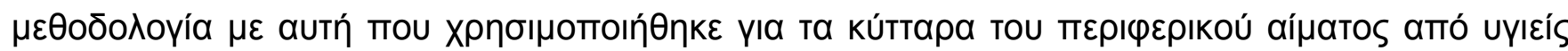

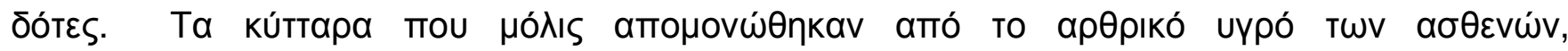

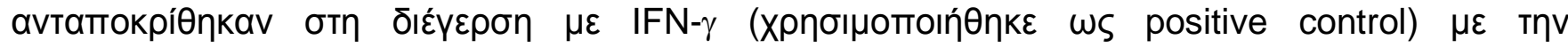

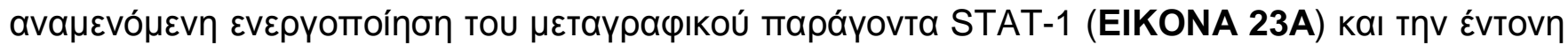

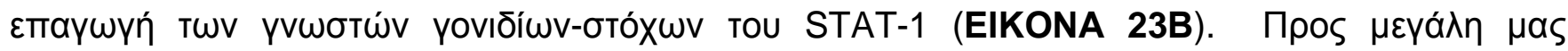

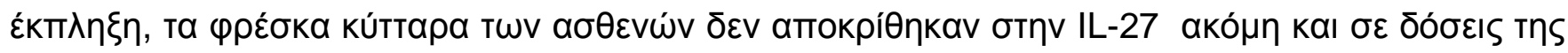

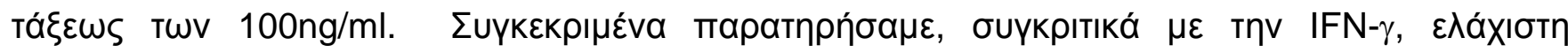




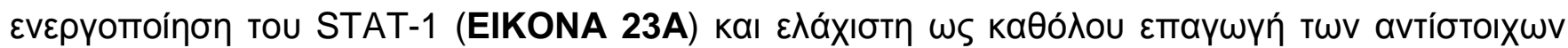

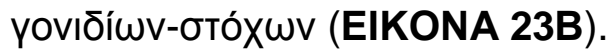

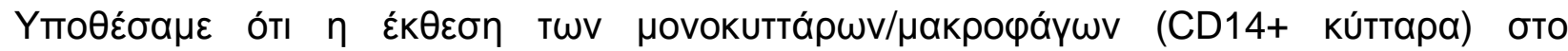

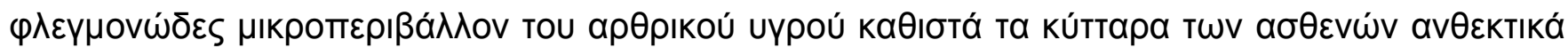

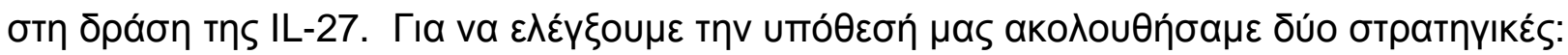

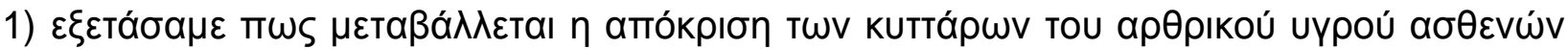

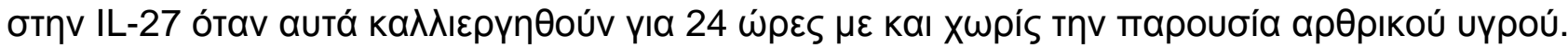

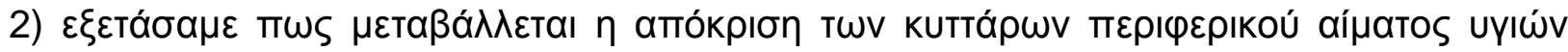

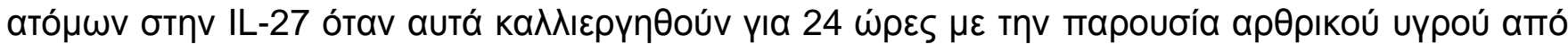

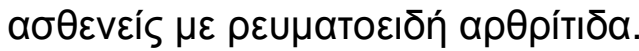

A

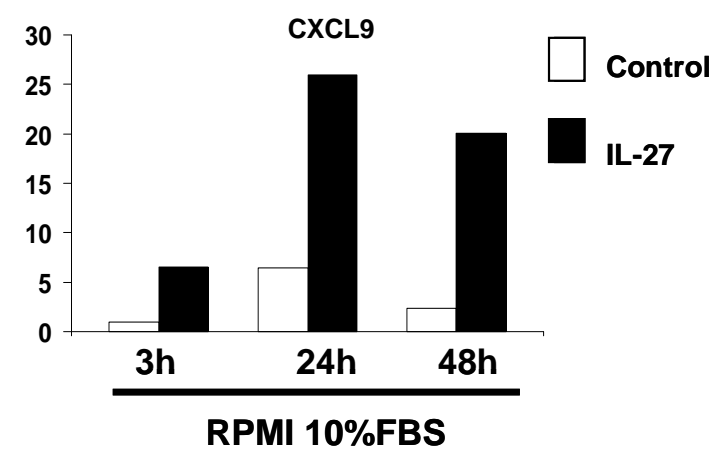

B

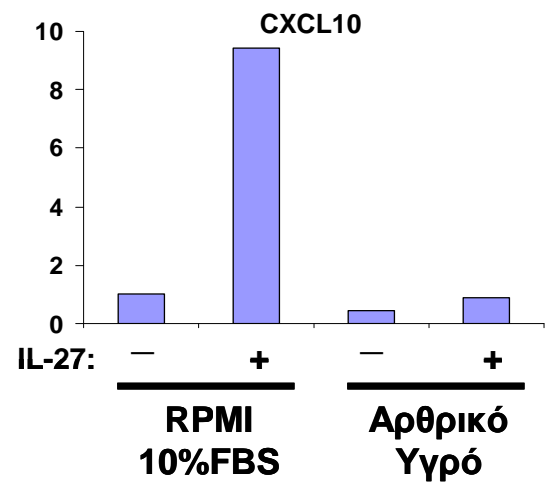

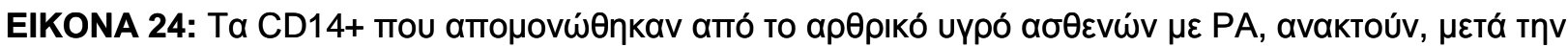

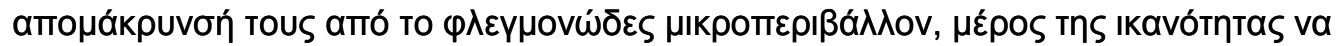

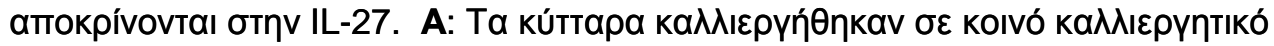

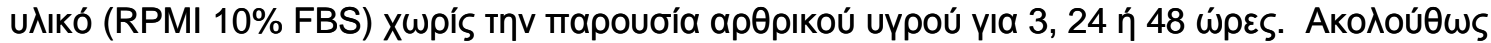

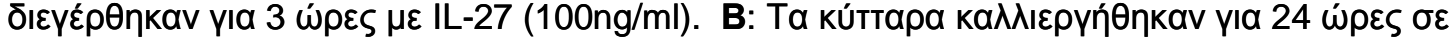

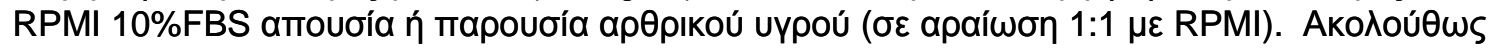

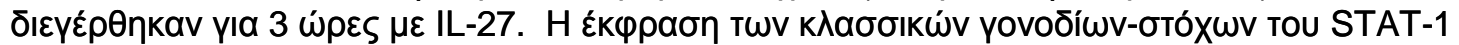

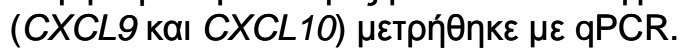




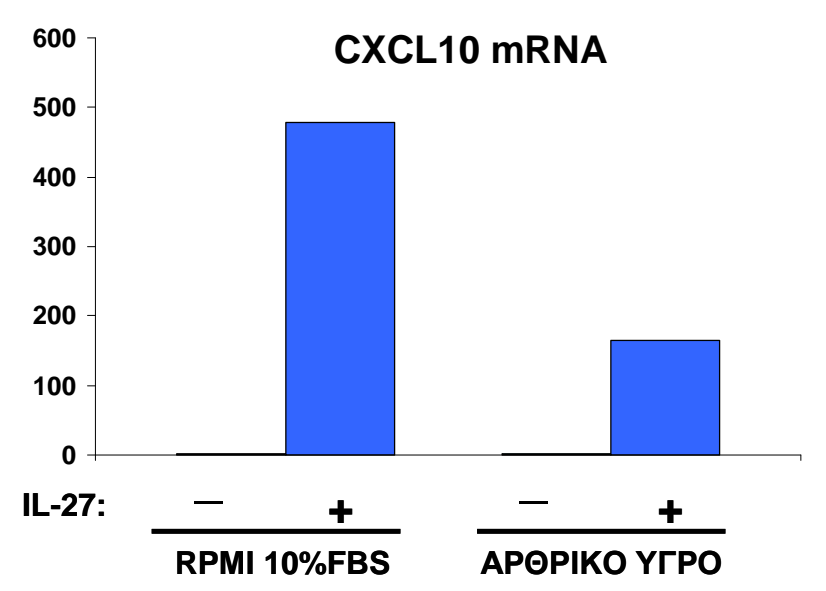

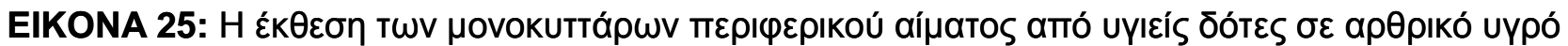

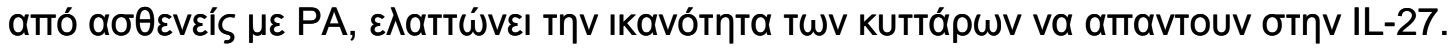

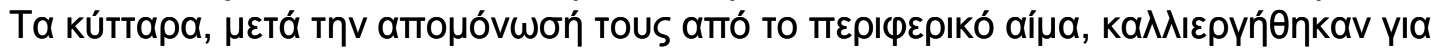

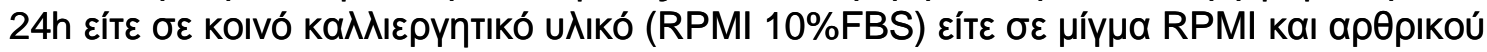

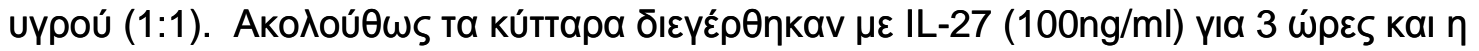

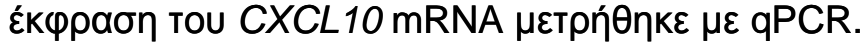

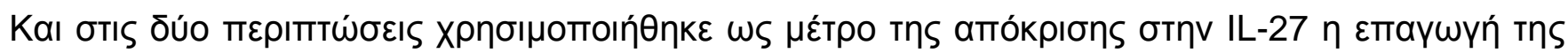

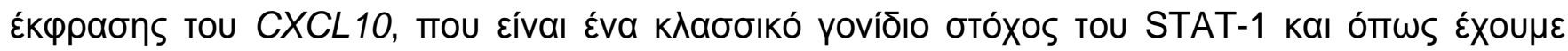

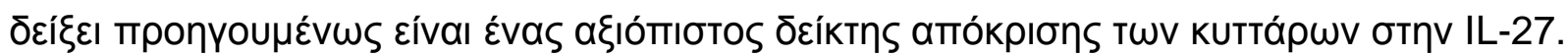

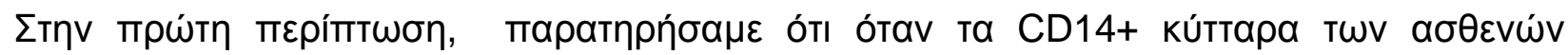

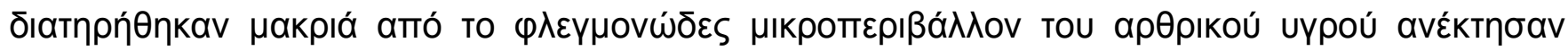

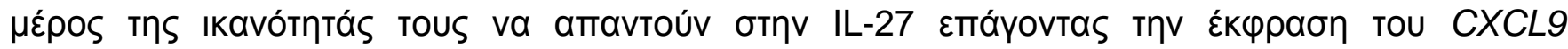

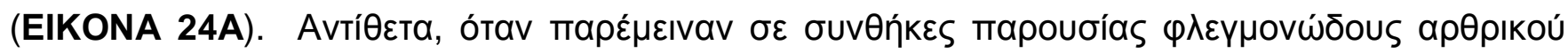

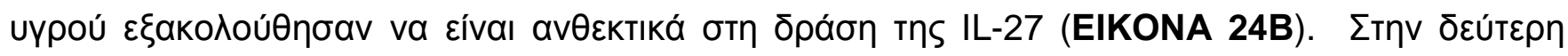

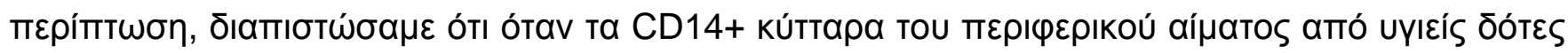

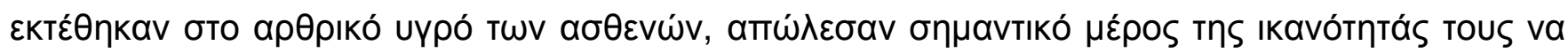

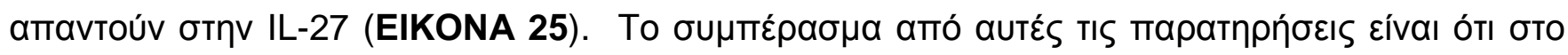

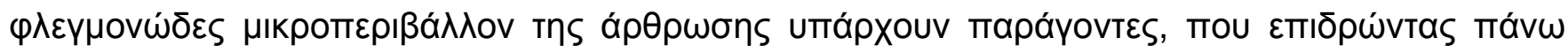

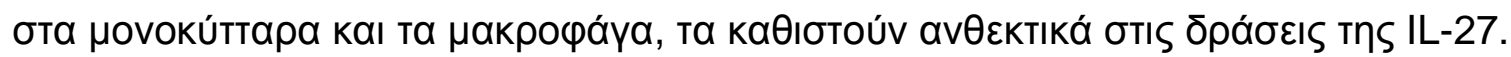




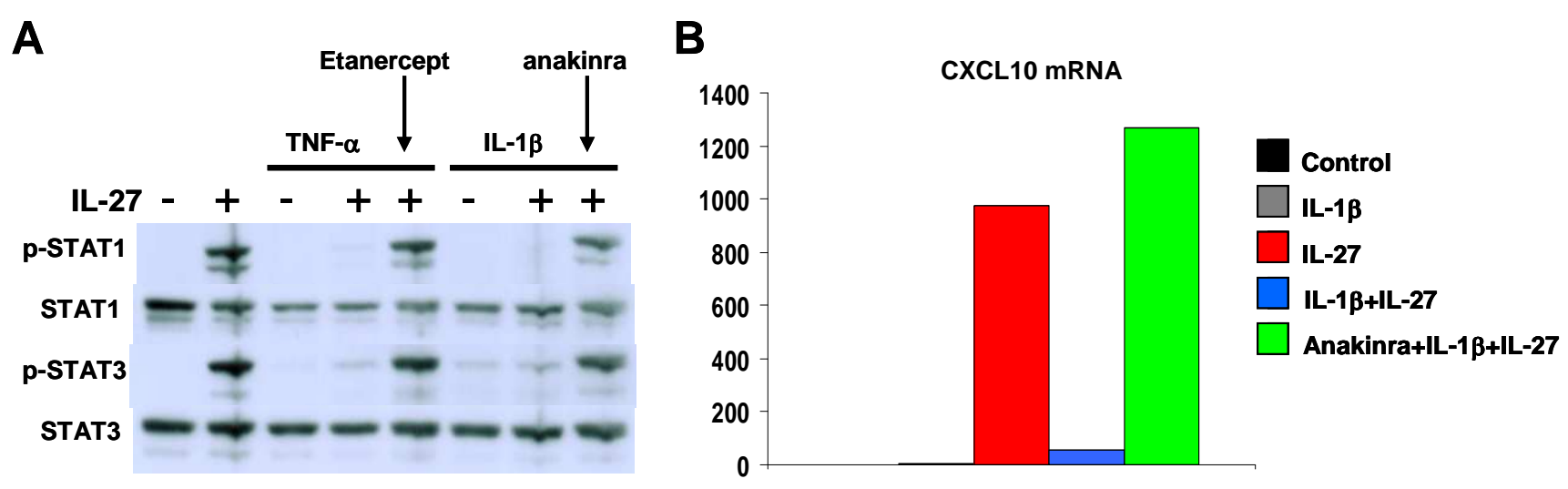

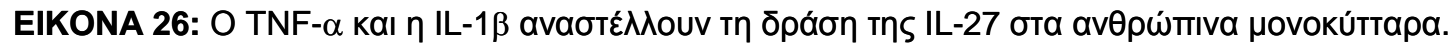

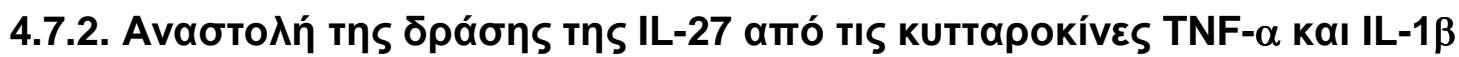

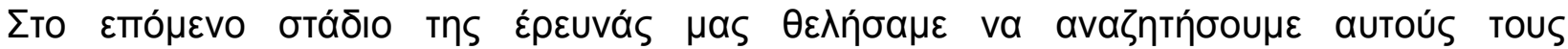

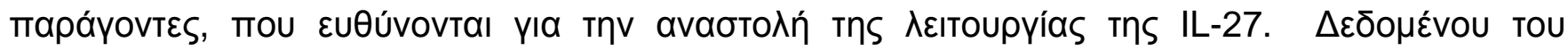

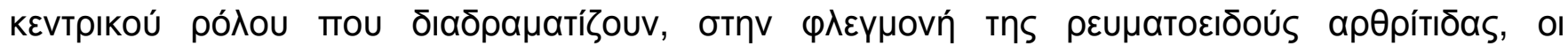

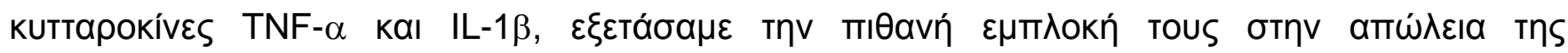

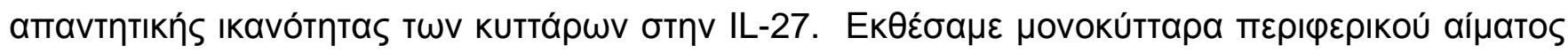

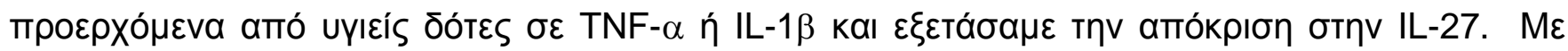

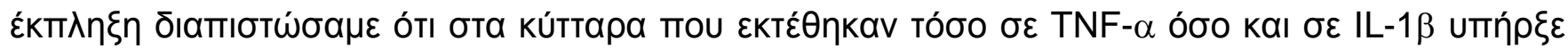

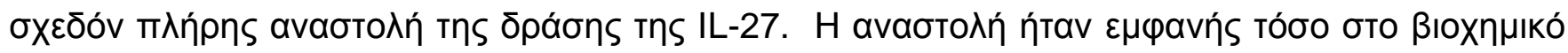

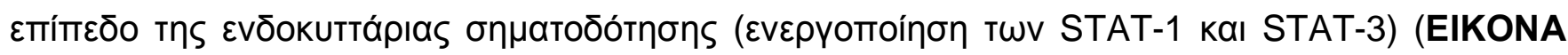

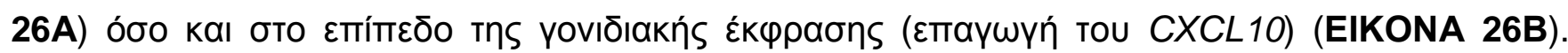

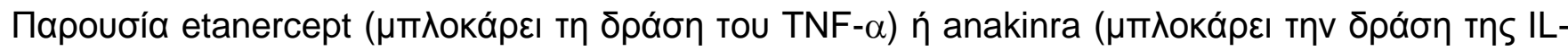

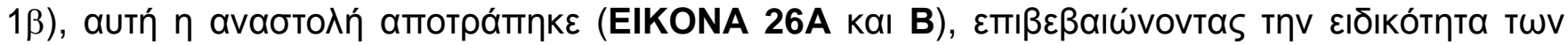

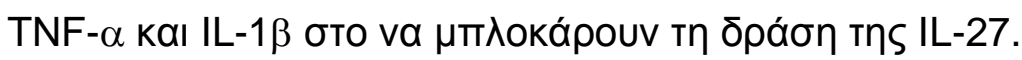


A

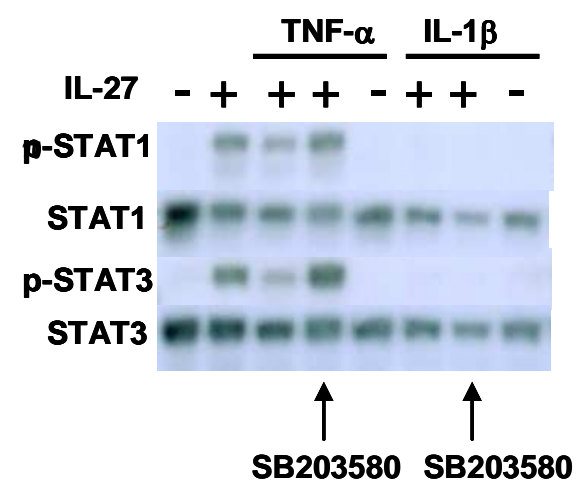

B

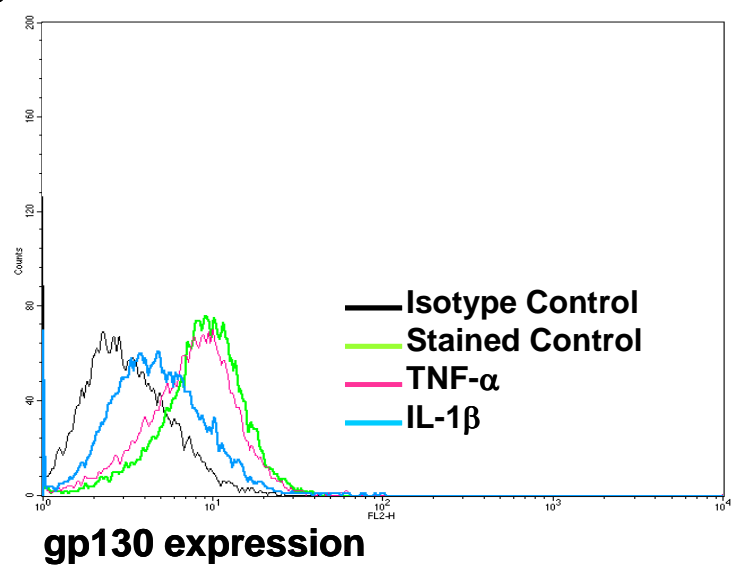

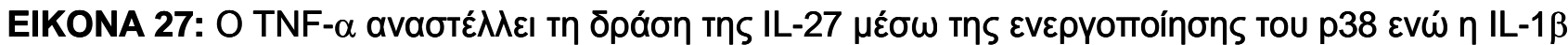

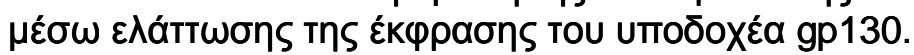

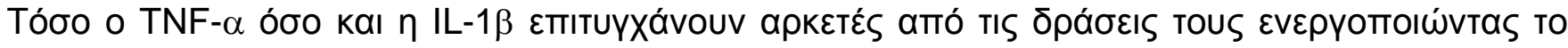

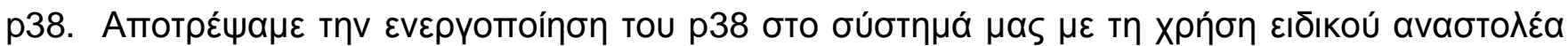

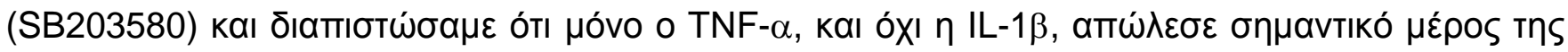

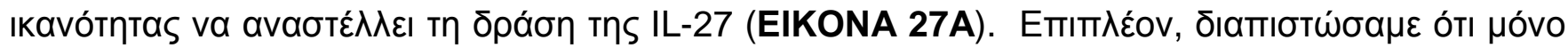

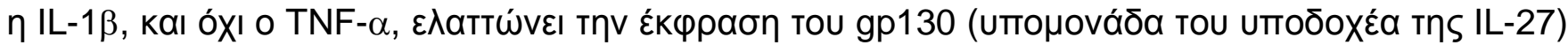

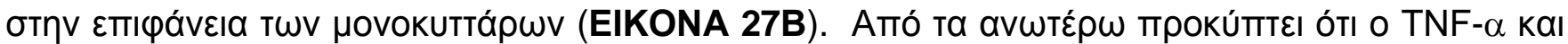

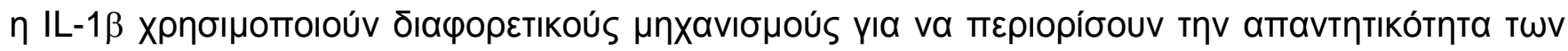

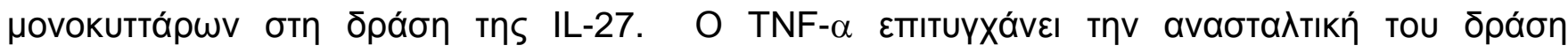

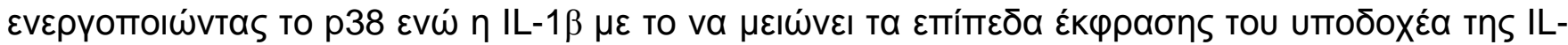

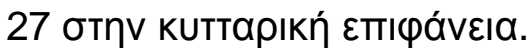


A

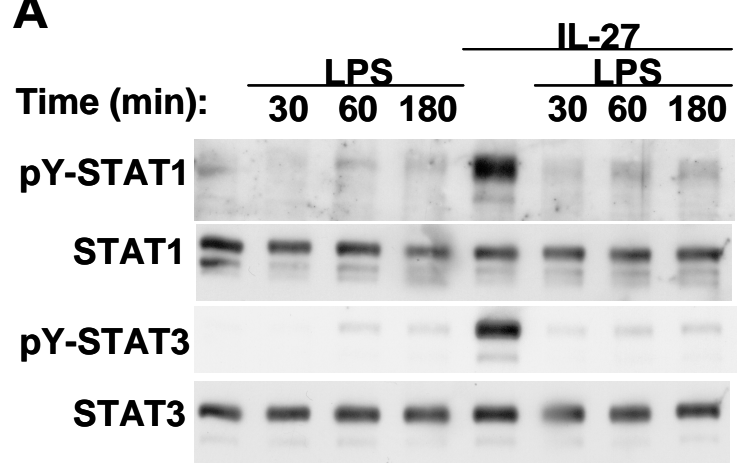

B

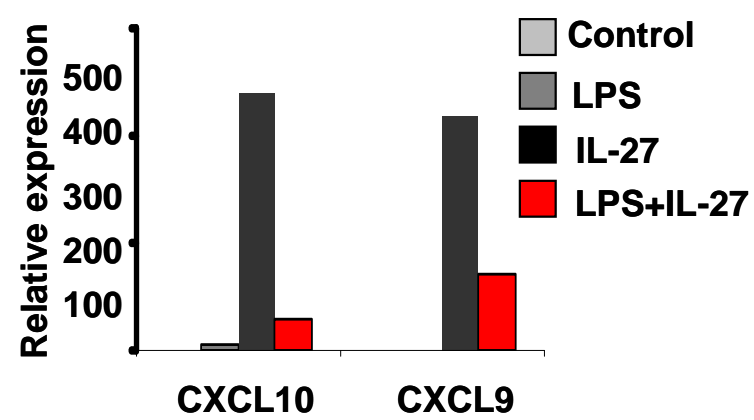

$\Gamma$

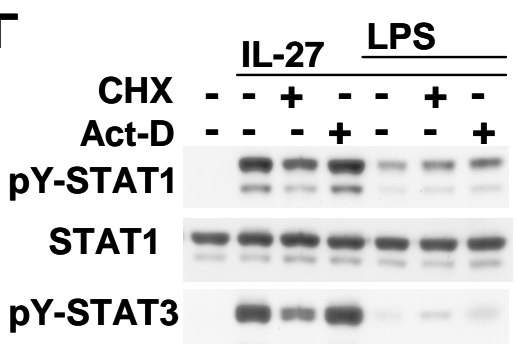

STAT3
$\Delta$

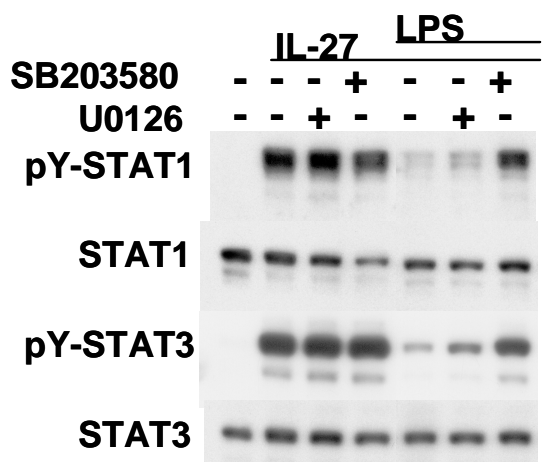

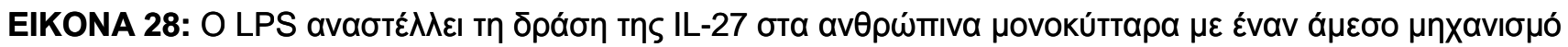

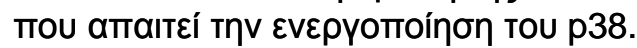

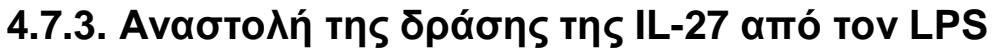

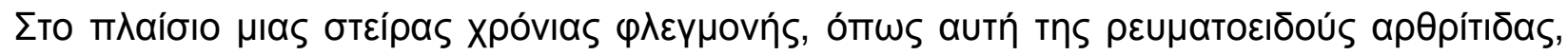

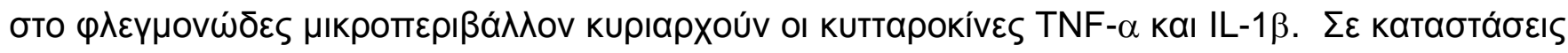

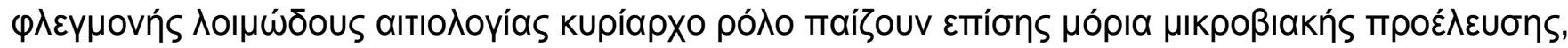

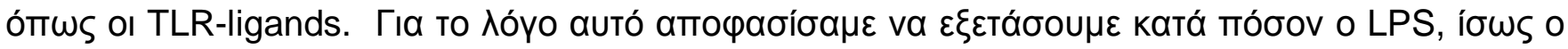

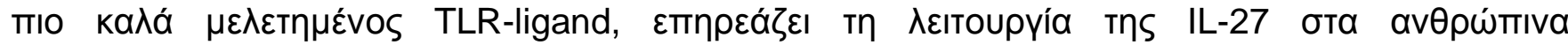

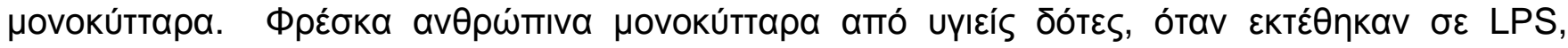

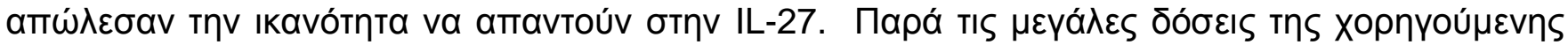

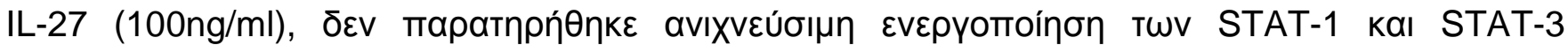

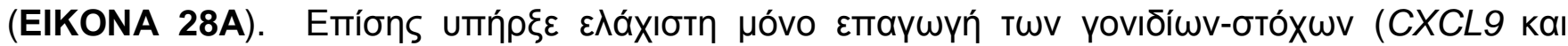




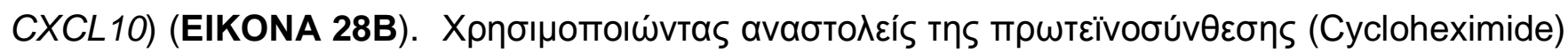

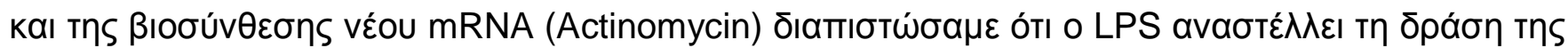

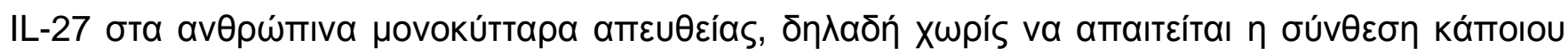

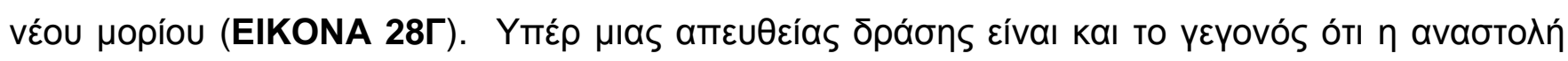

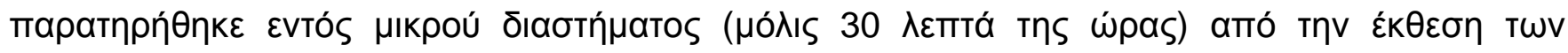

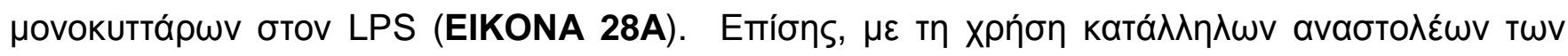

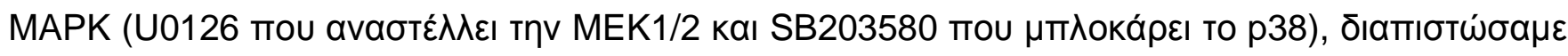

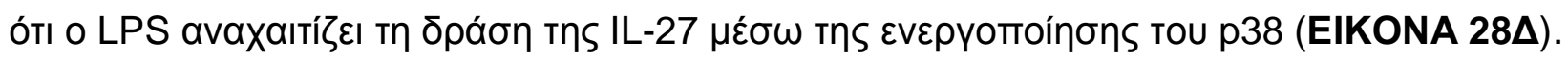

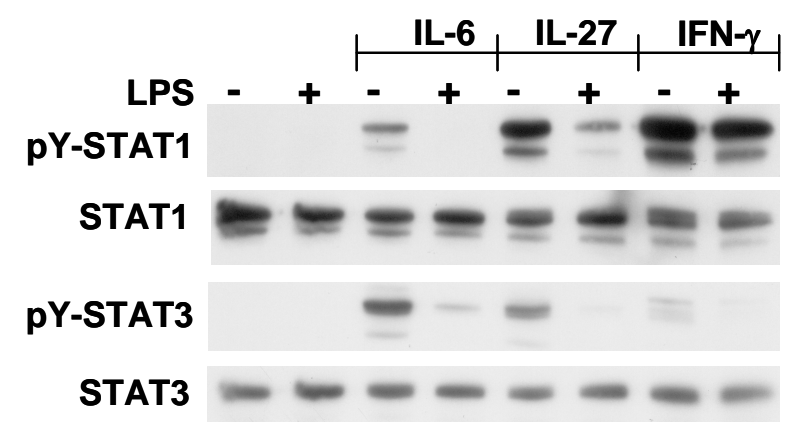

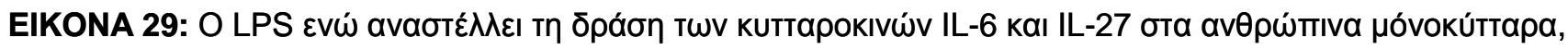

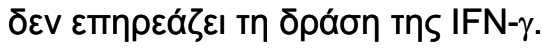

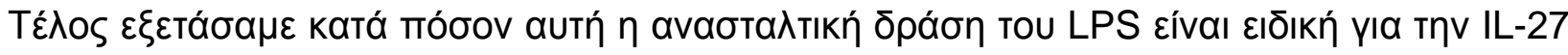

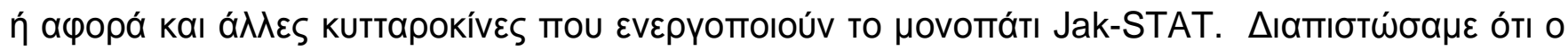

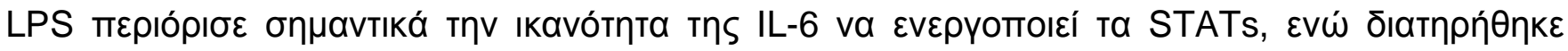

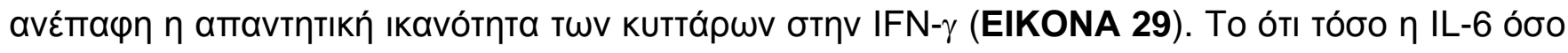

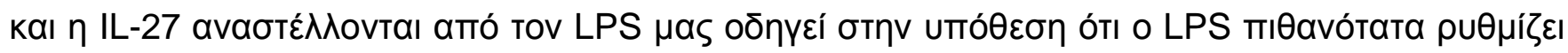

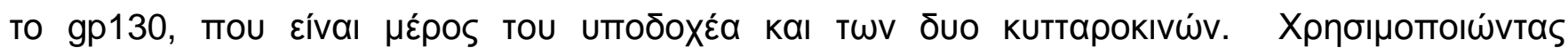

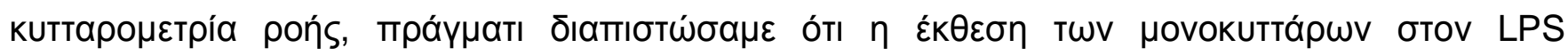

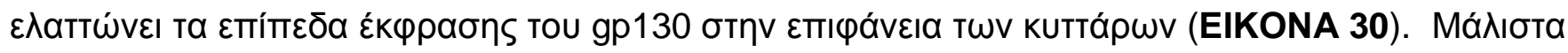

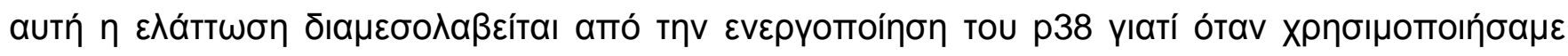




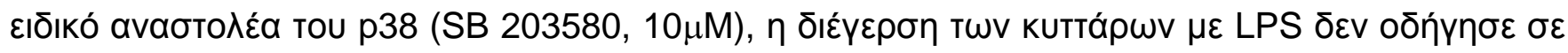

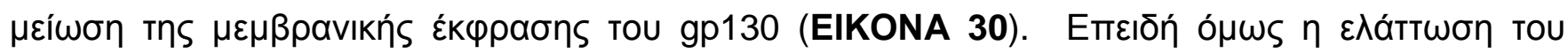

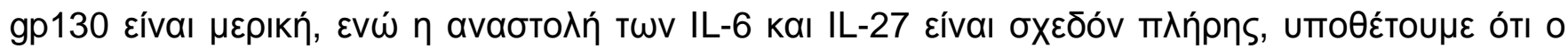

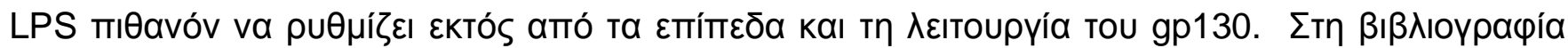

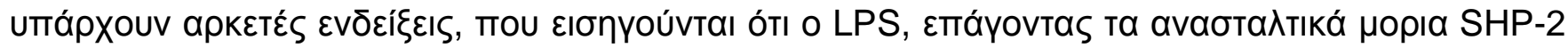

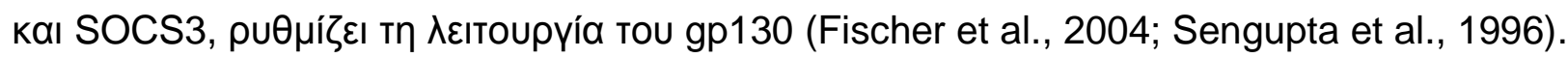

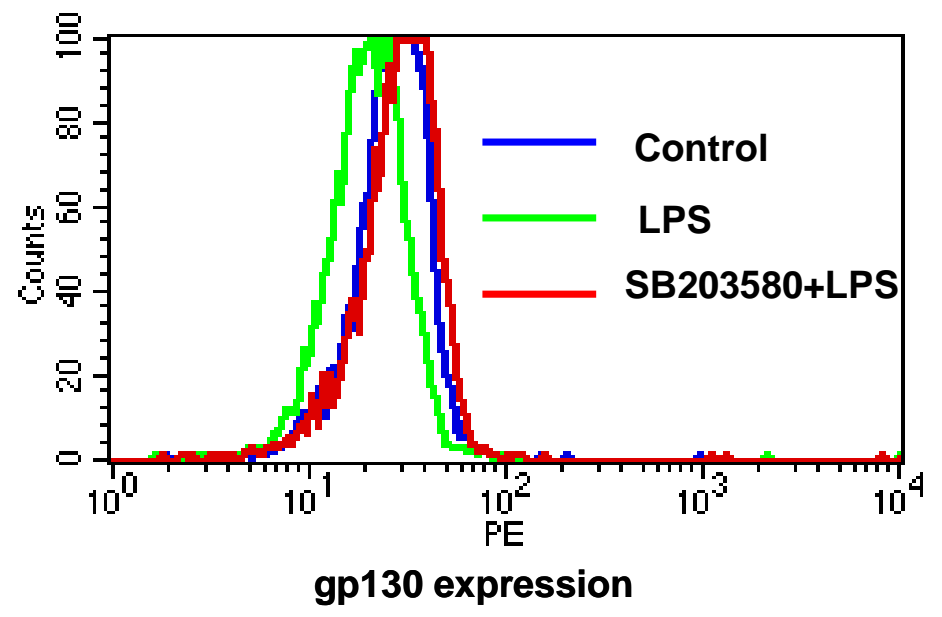

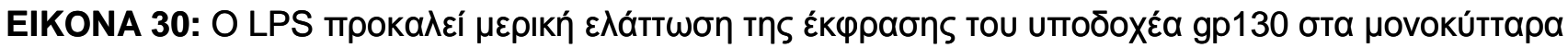

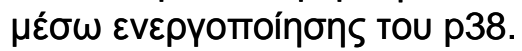




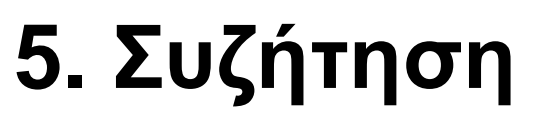




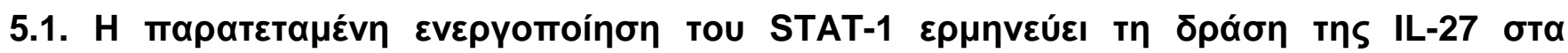

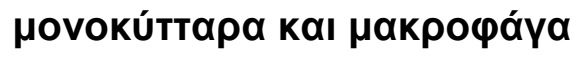

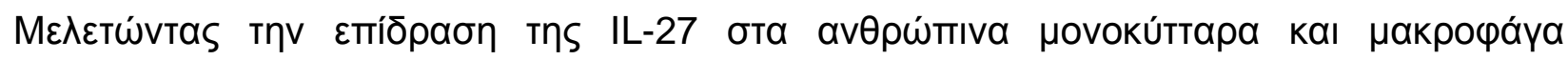

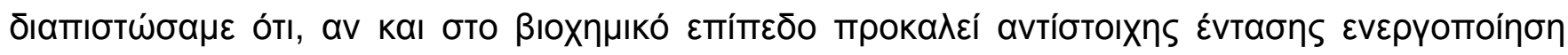

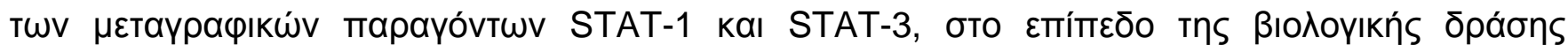

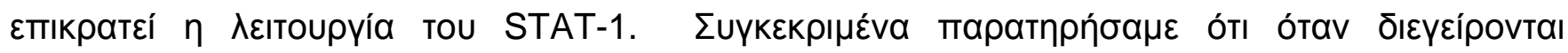

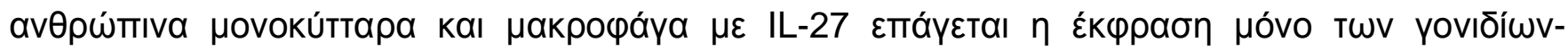

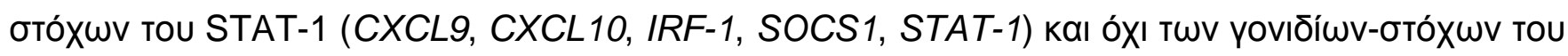

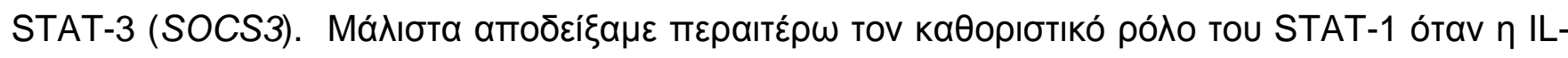

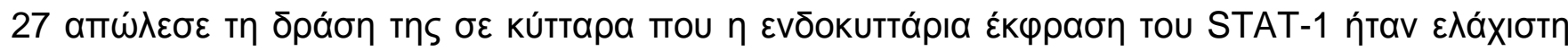
(THP-1 STAT-1 Low).

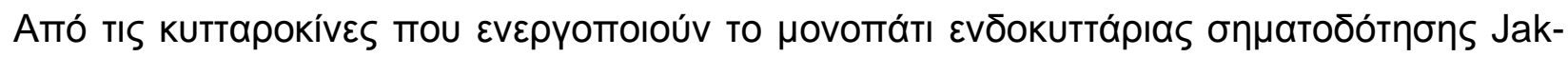

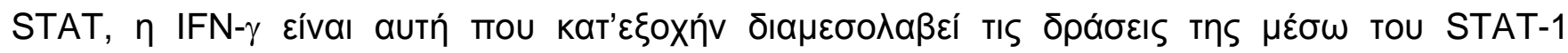

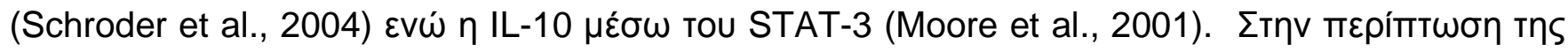

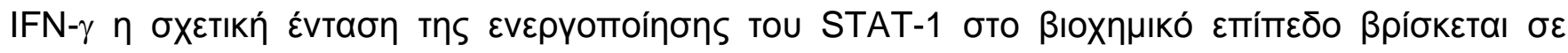

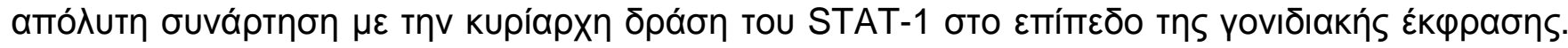

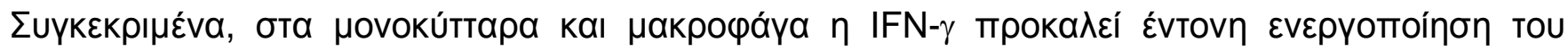

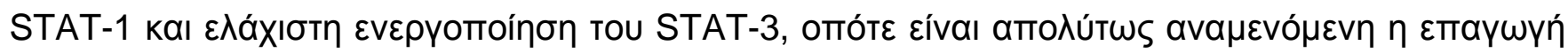

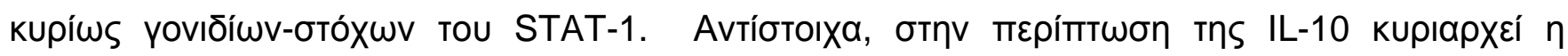

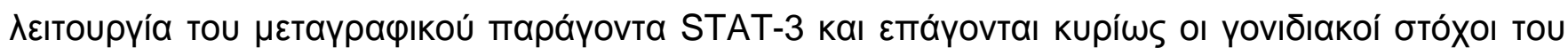

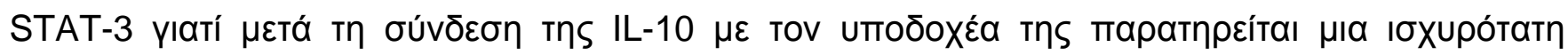

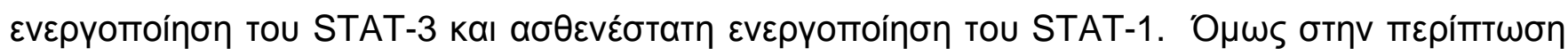

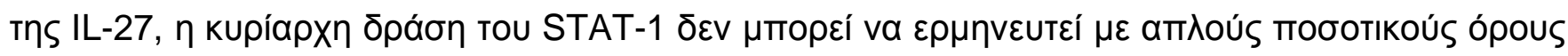




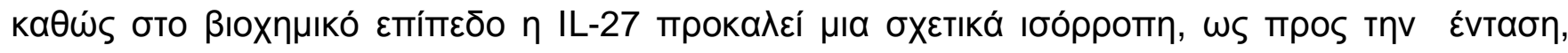

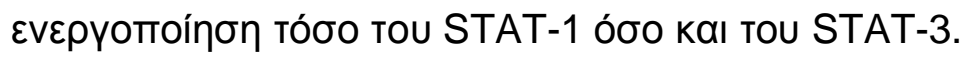

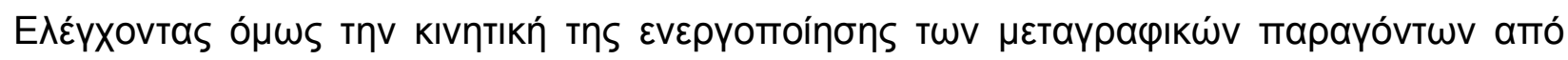

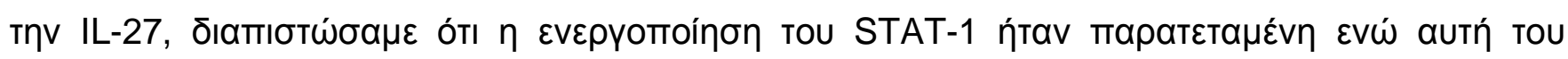

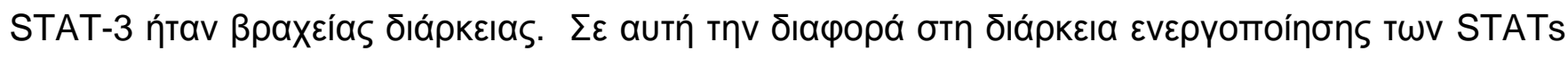

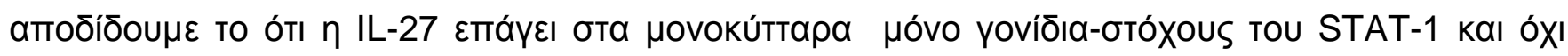

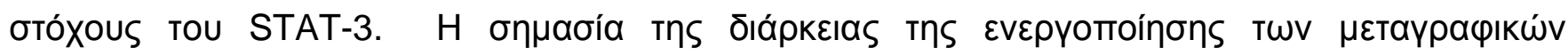

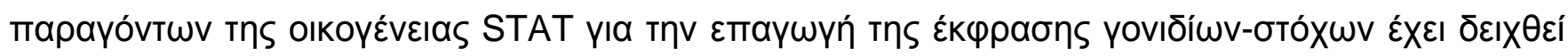

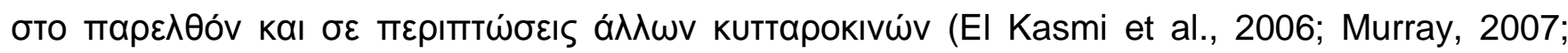
Yoshimura et al., 2007). 


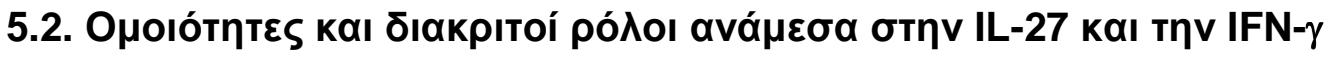

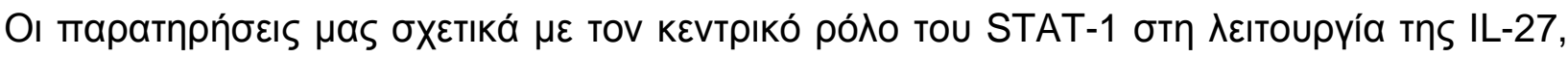

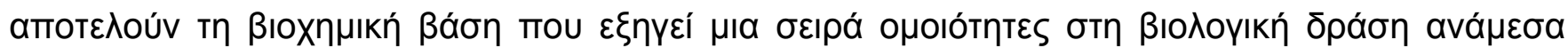

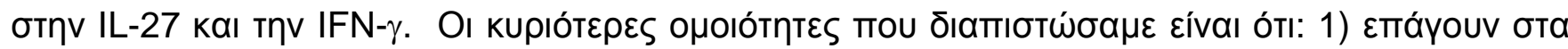

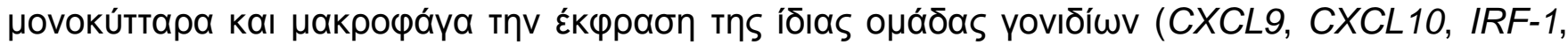

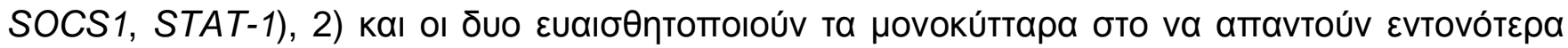

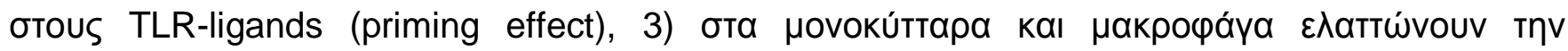

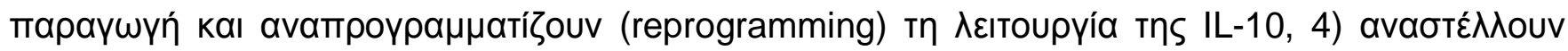

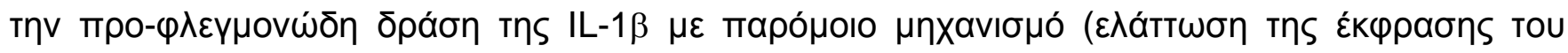

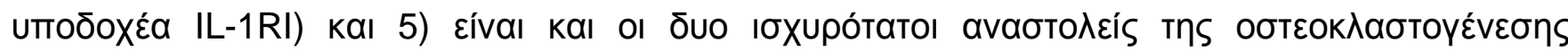
(Herrero et al., 2003; Hu et al., 2005; Hu et al., 2006; Takayanagi et al., 2000). Оı оноіо́тптৎ

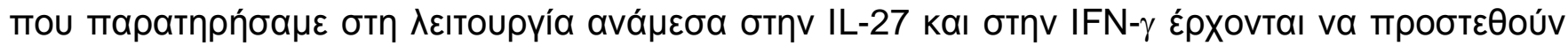

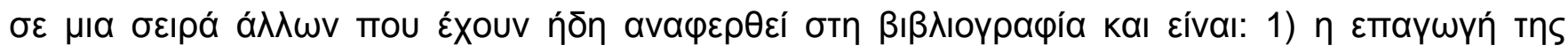

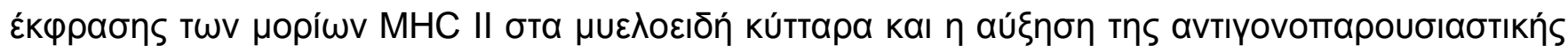

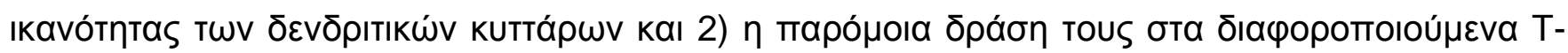

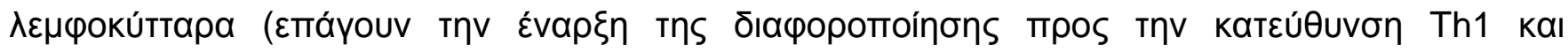

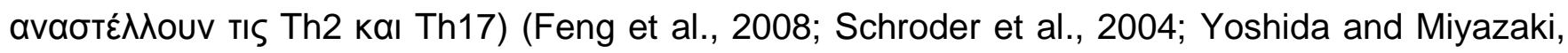
2008).

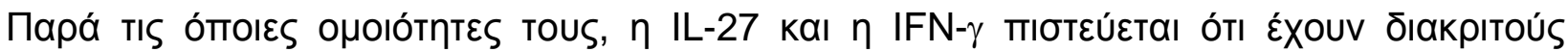

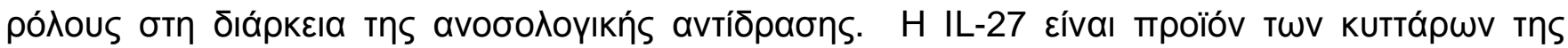

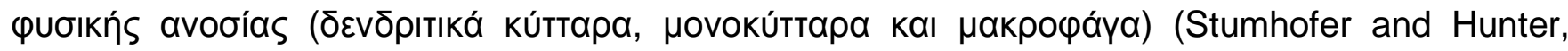

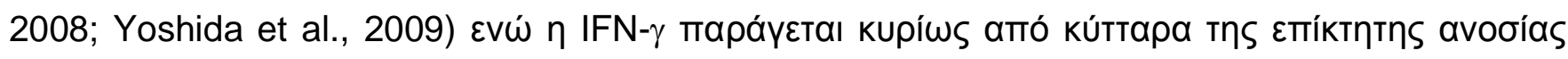

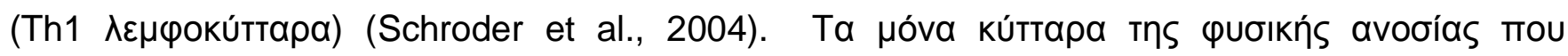




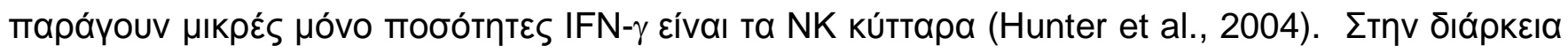

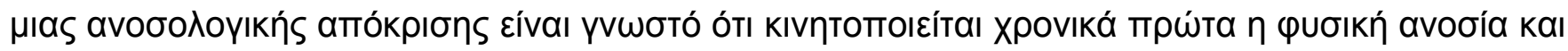

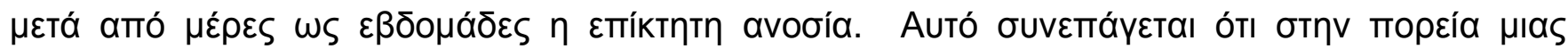

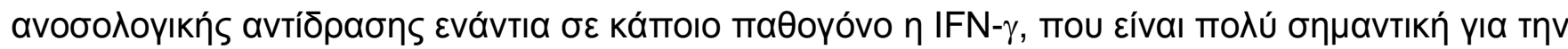

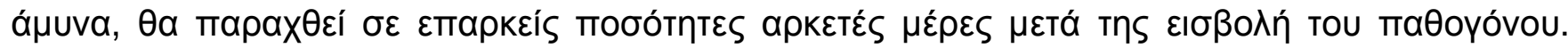

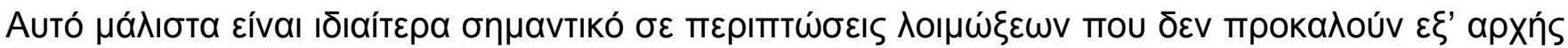

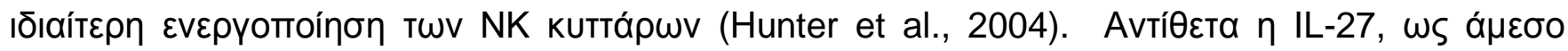

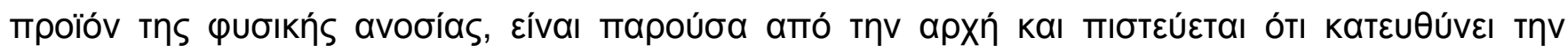

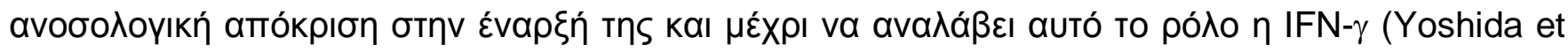

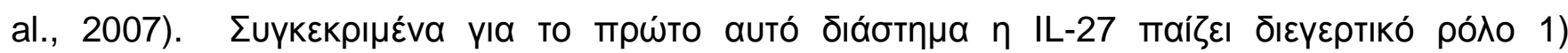

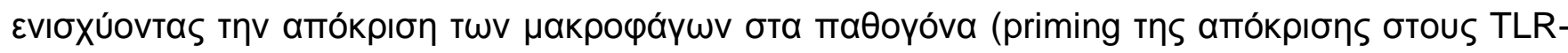

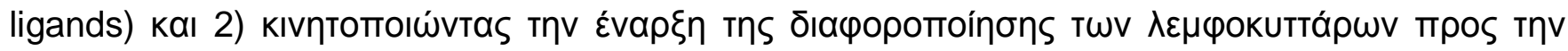

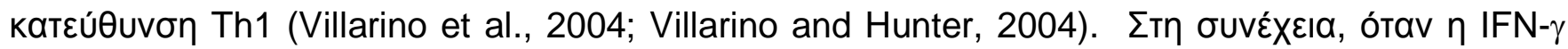

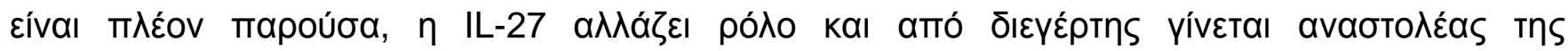

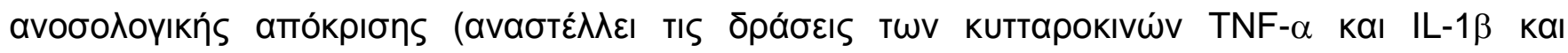

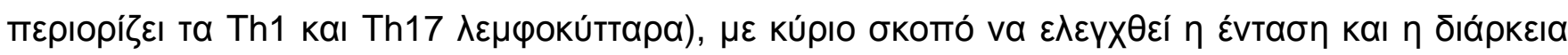

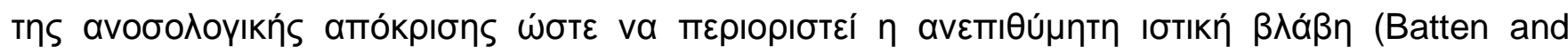
Ghilardi, 2007; Stumhofer and Hunter, 2008; Yoshida et al., 2009). 


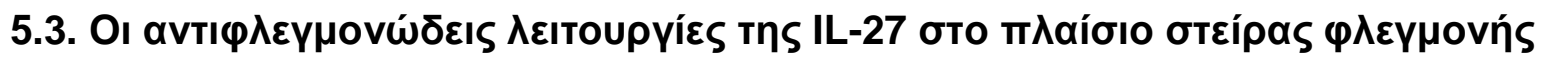

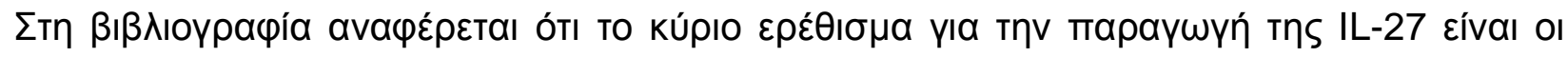
TLR-ligands каı та таӨoyóva (Goriely et al., 2008; Stumhofer and Hunter, 2008; Yoshida et al.,

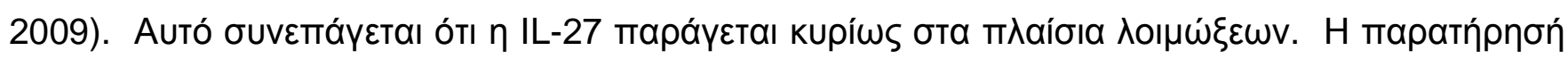

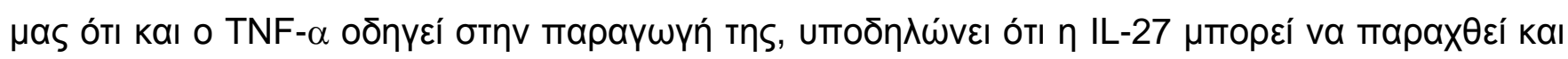

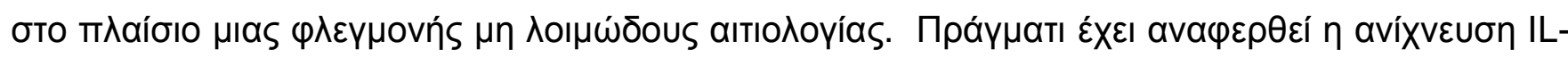

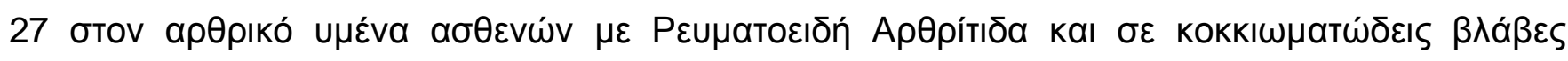

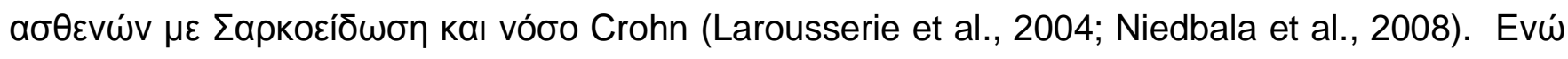

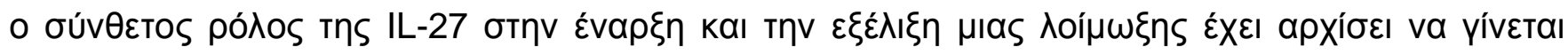

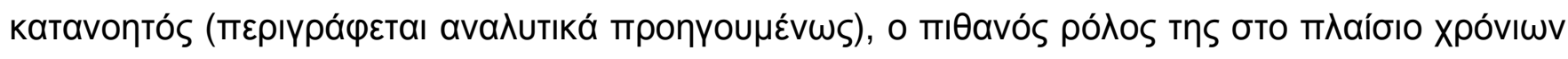

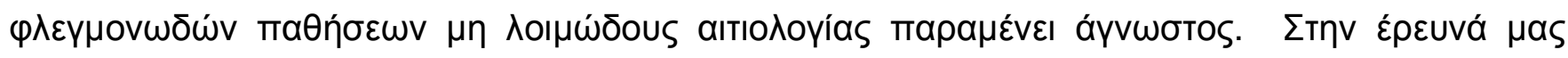

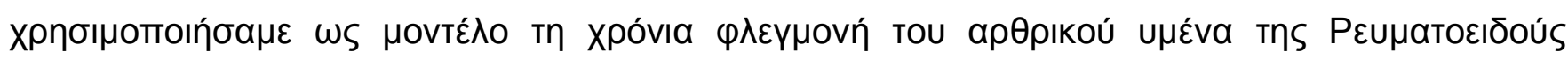

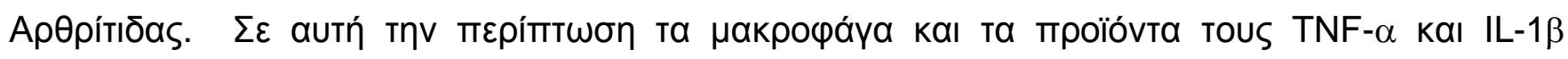

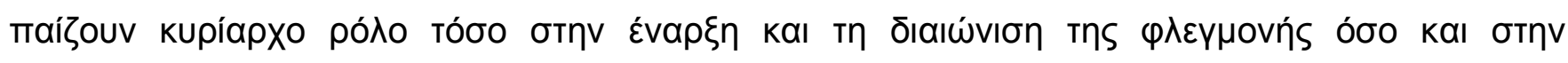

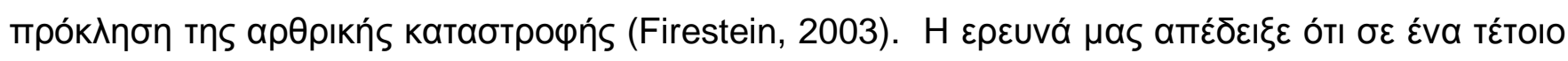

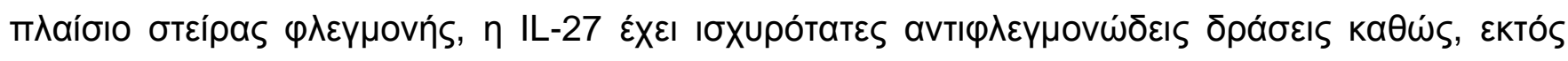

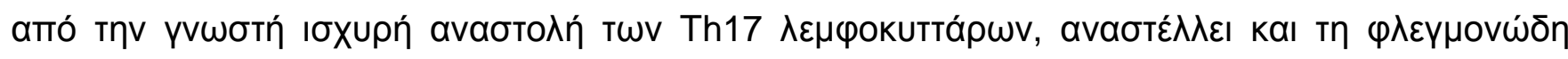

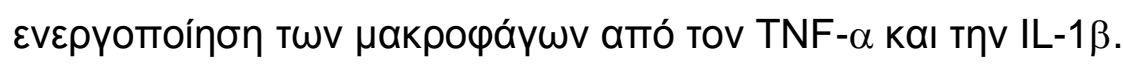

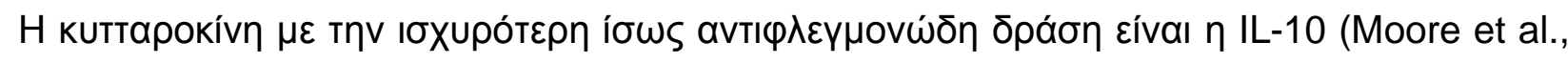

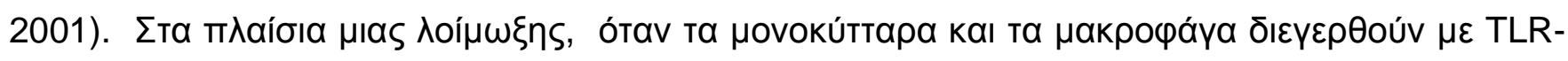

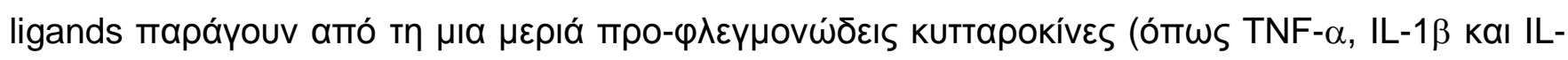

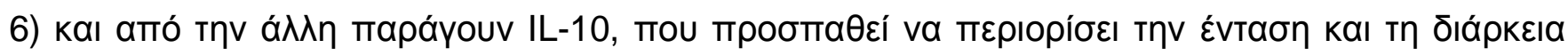

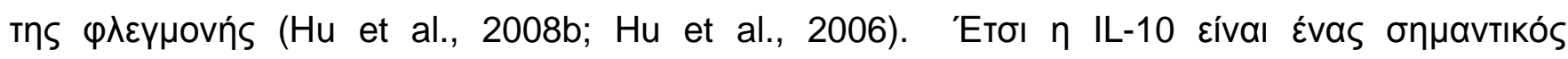




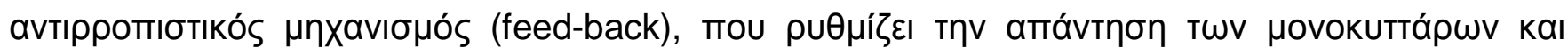

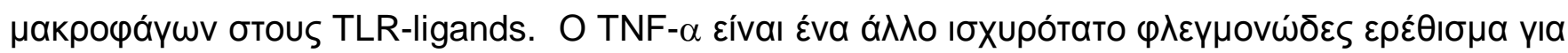

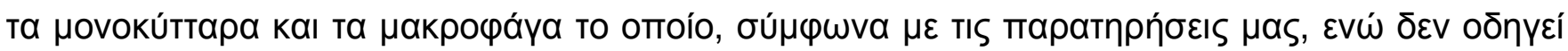

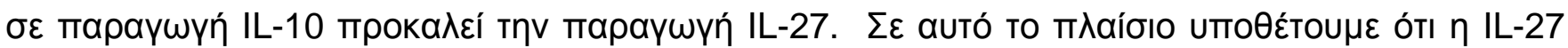

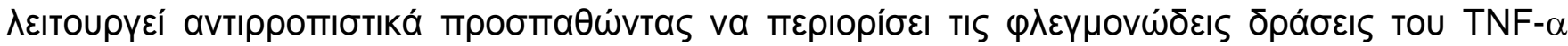

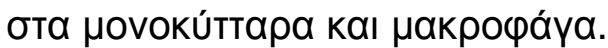




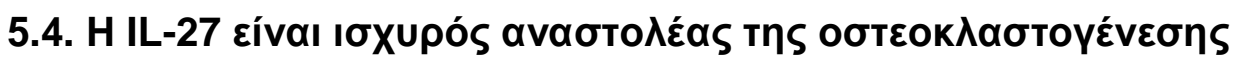

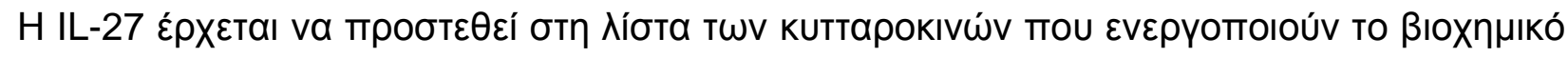

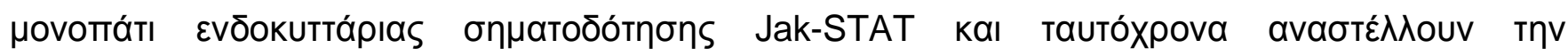

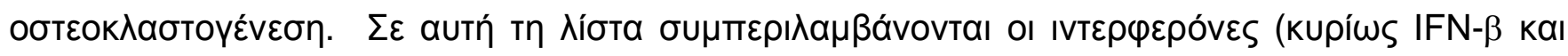
IFN- $\gamma$ ) (Takayanagi et al., 2002b; Takayanagi et al., 2002c; Takayanagi et al., 2000), ๆ IL-4

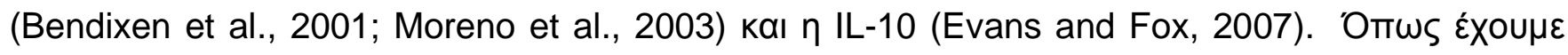

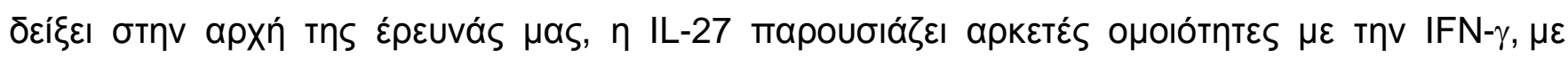

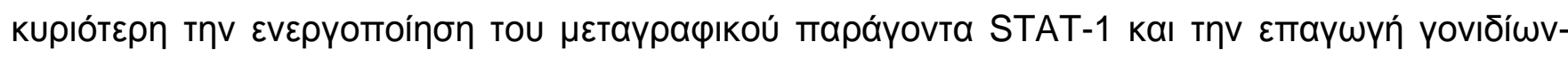

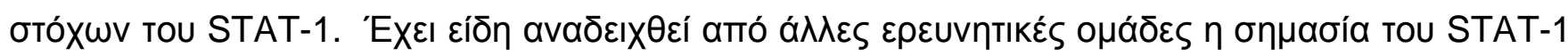

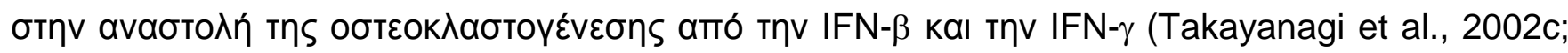

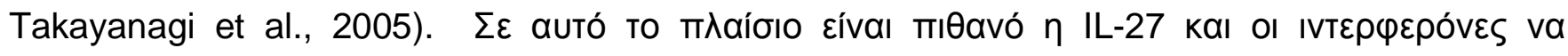

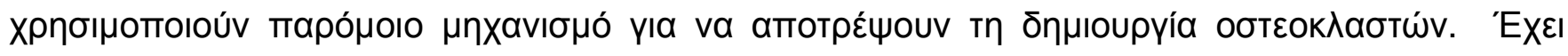

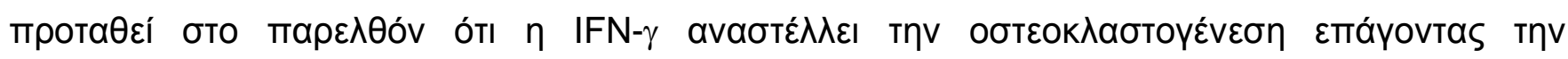

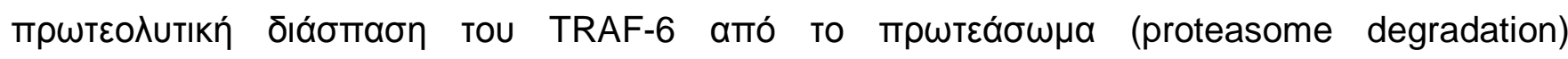

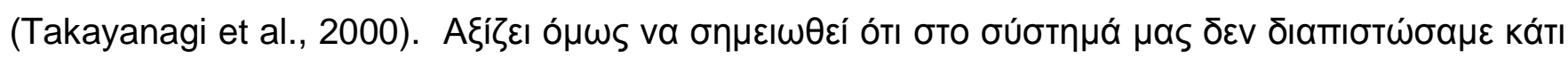

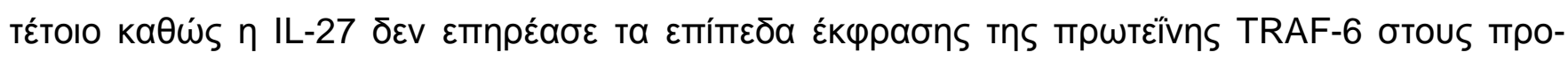

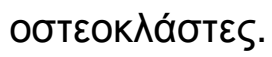

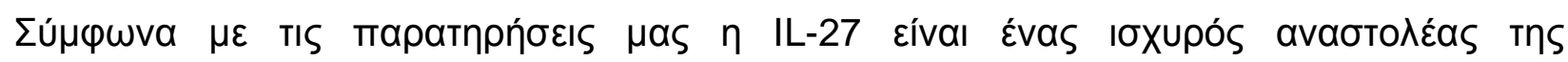

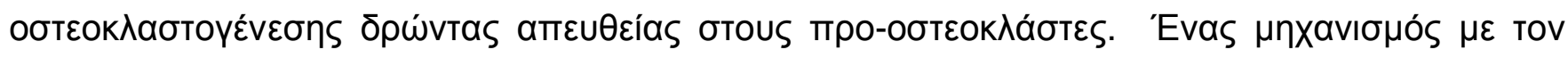

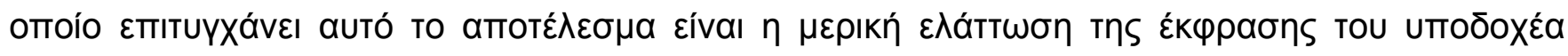

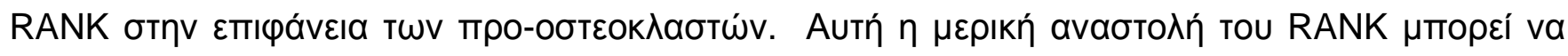

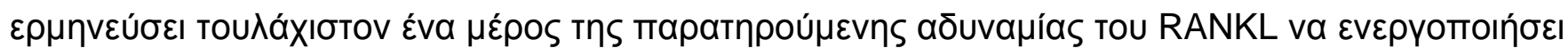

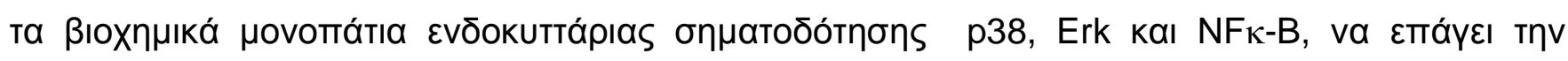




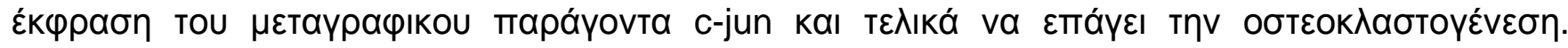

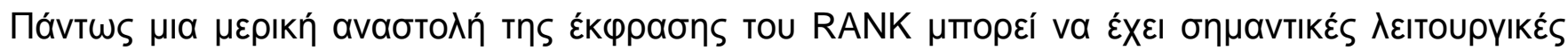

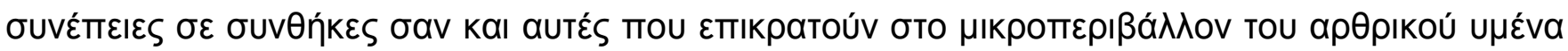

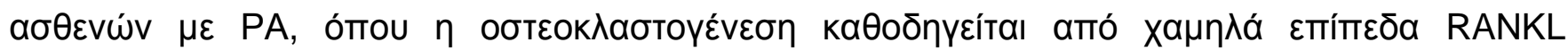

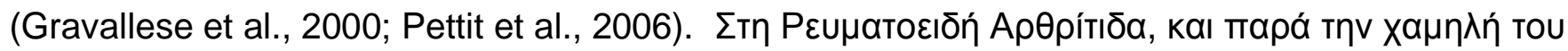

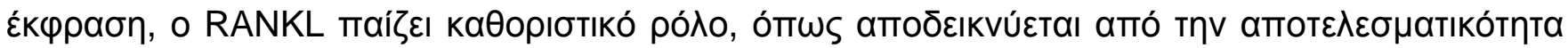
тоu denosumab ( катабтрофńs (Cohen et al., 2008).

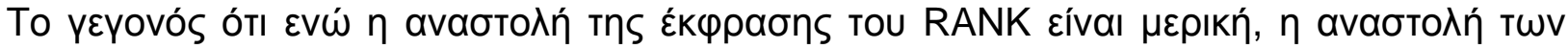

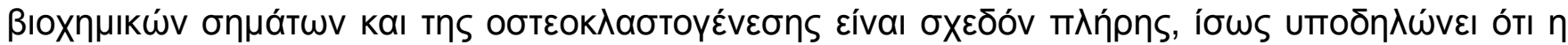

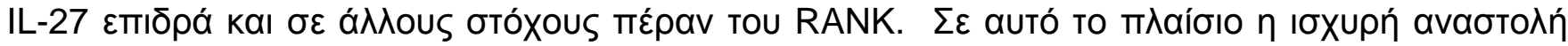

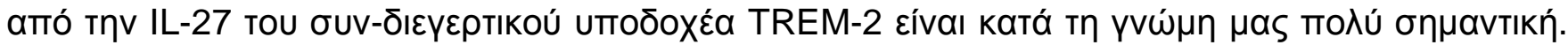

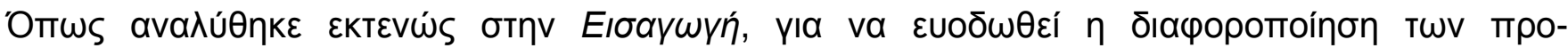

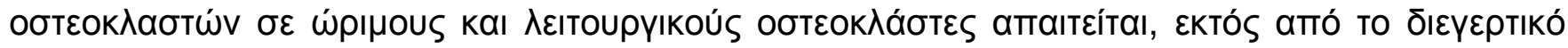

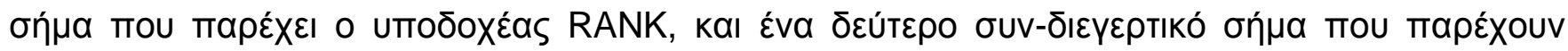

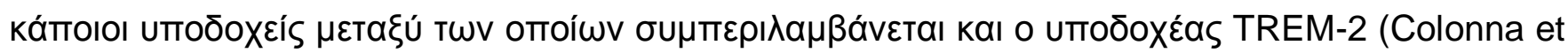

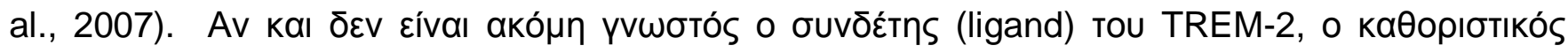

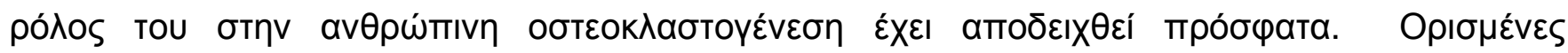

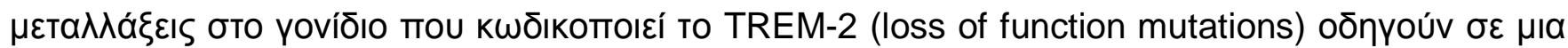

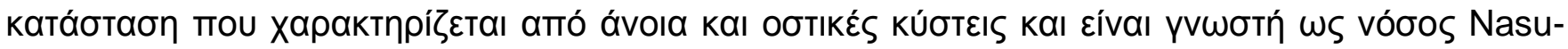

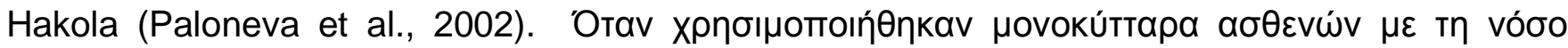

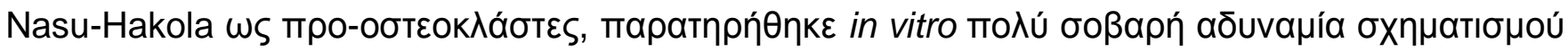

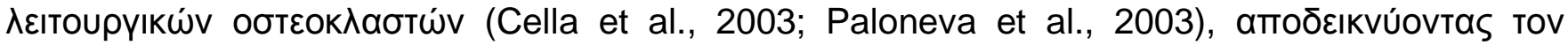




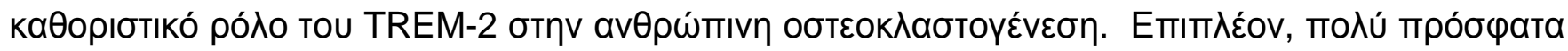

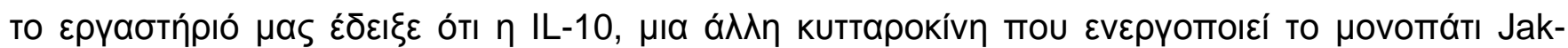

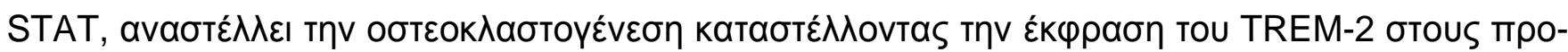

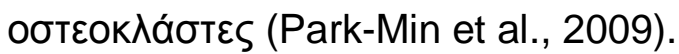

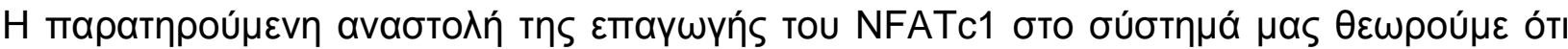

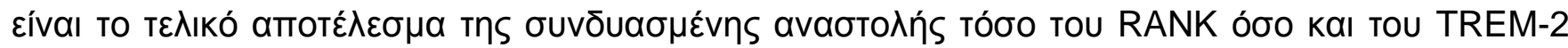

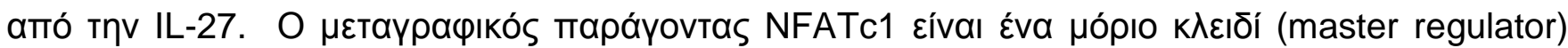

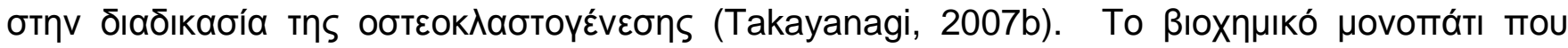

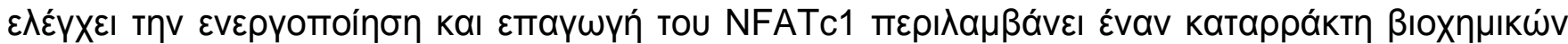

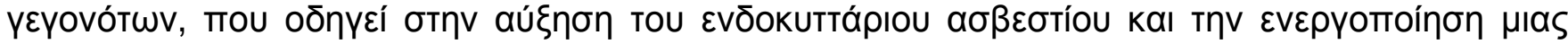

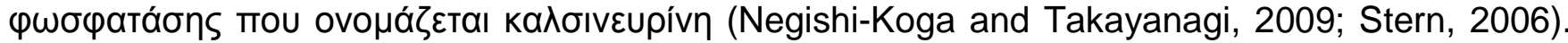

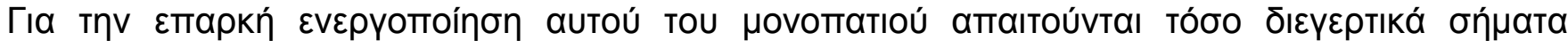

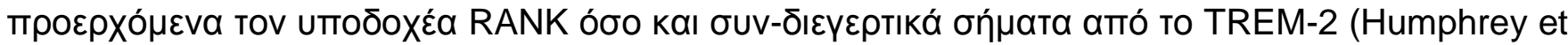

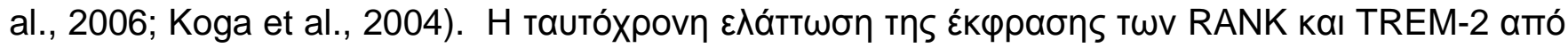

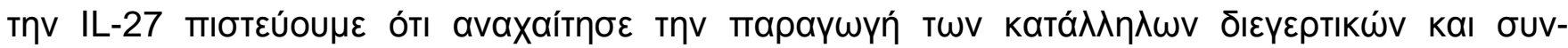

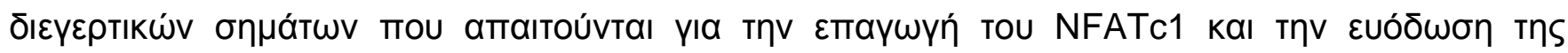

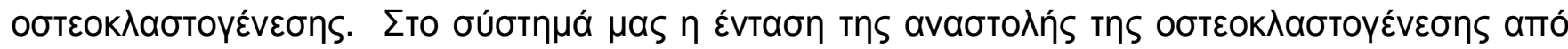

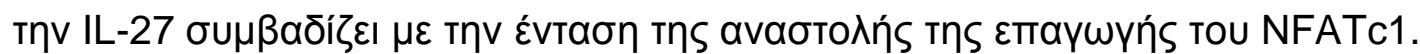

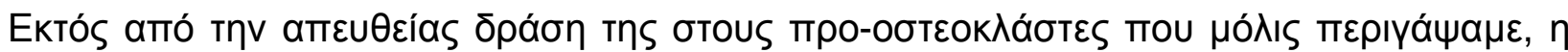

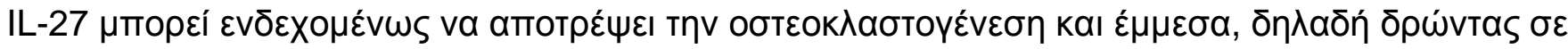

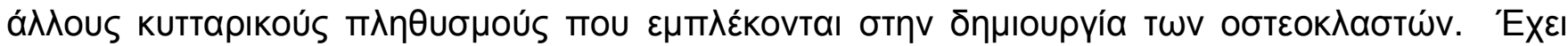

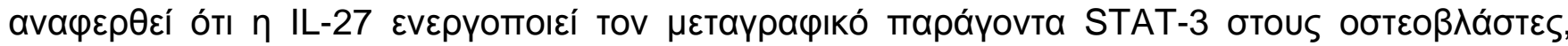

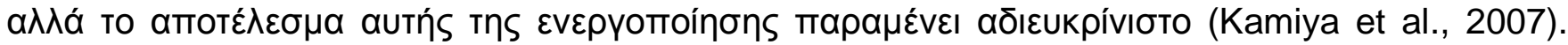




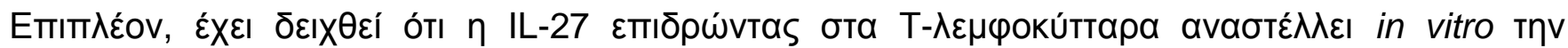

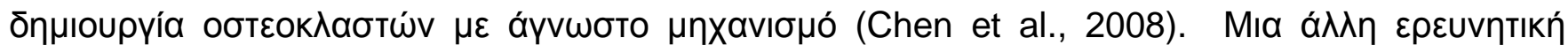

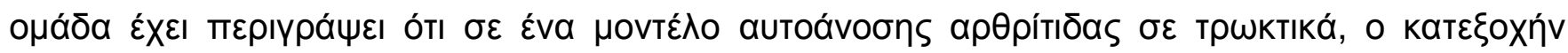

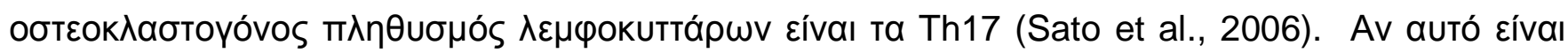

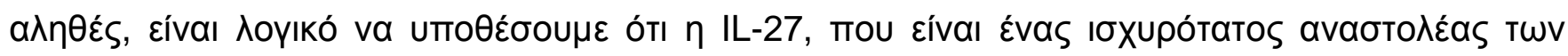

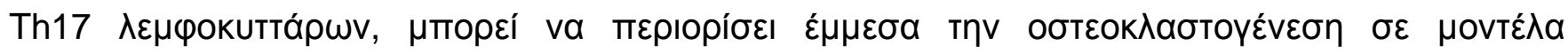

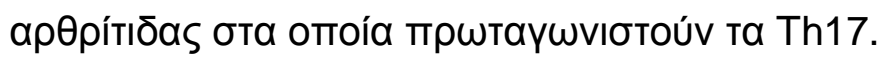




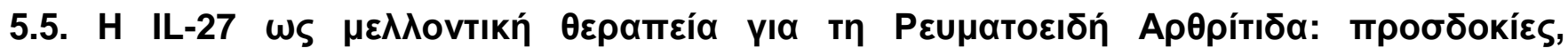

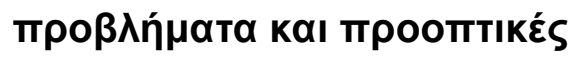

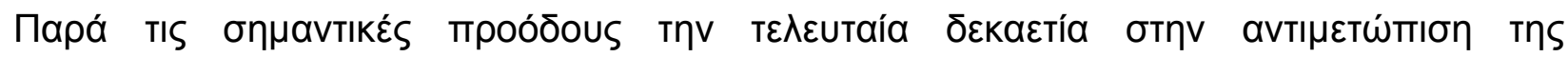

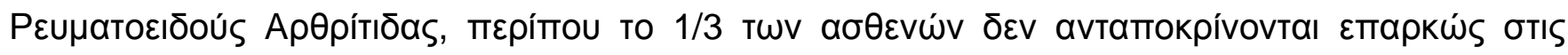

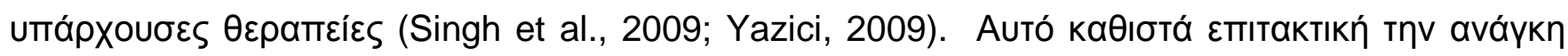

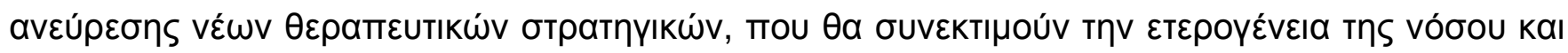

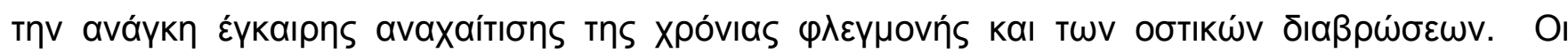

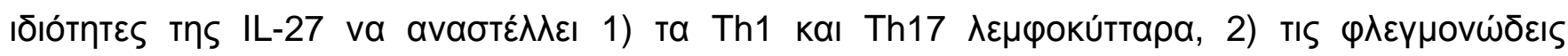

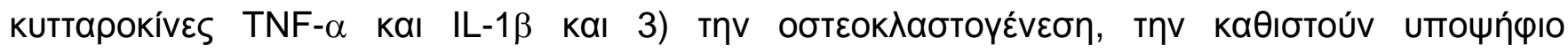

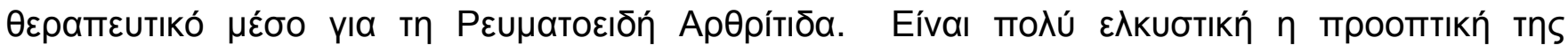

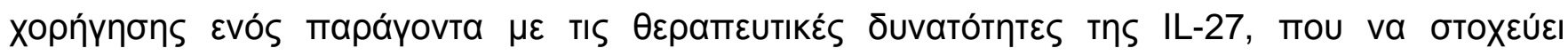

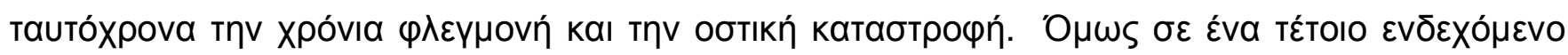

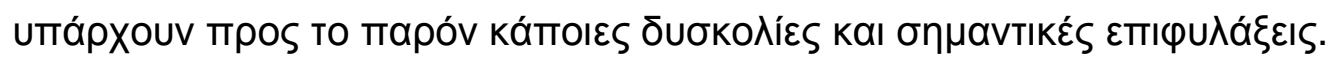

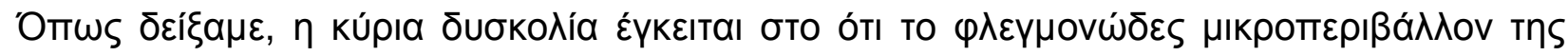

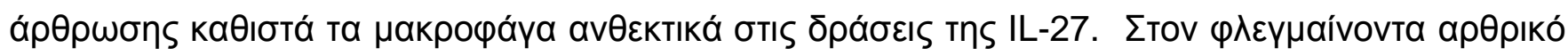

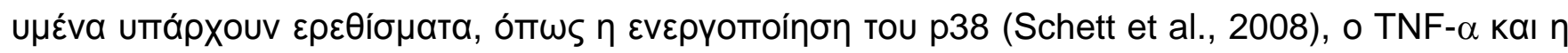

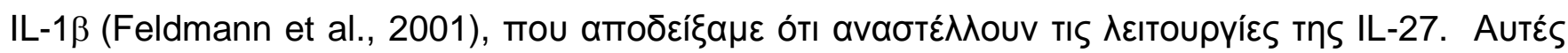

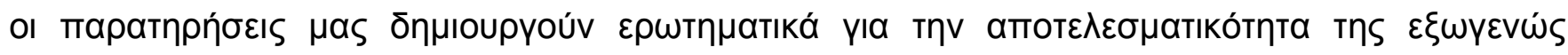

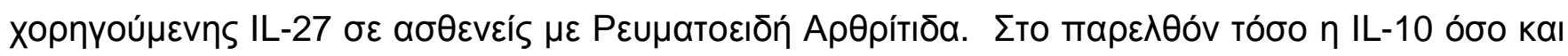

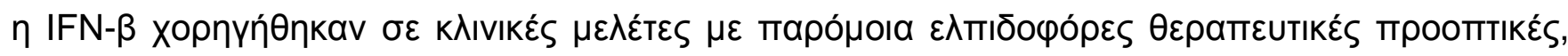

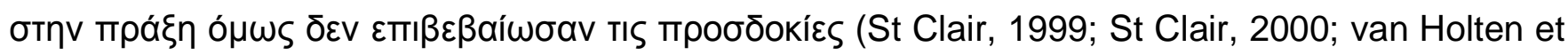

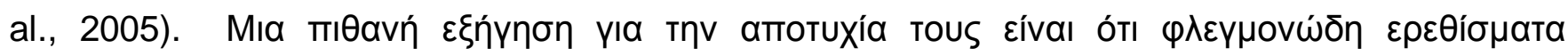

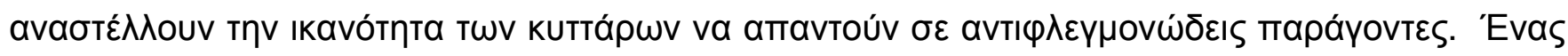




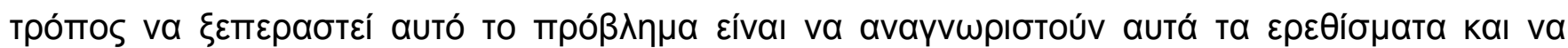

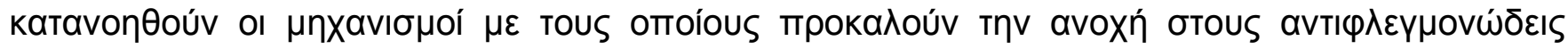

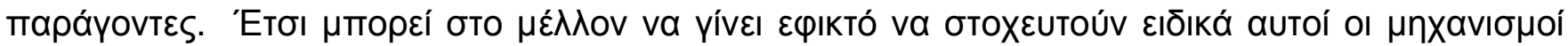

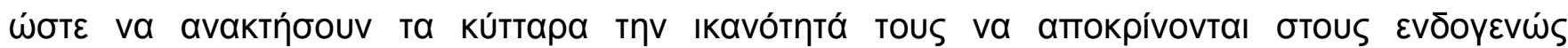

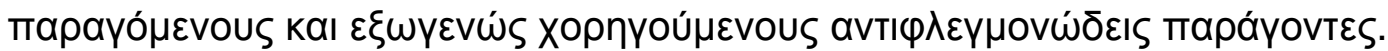

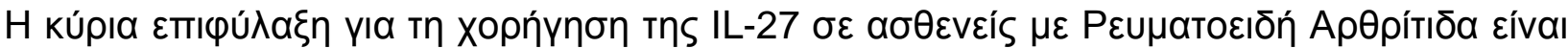

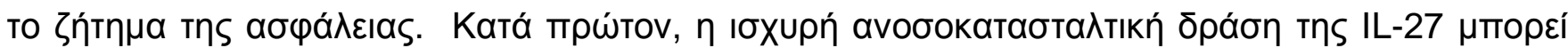

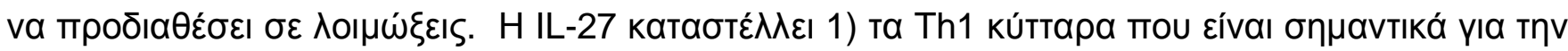

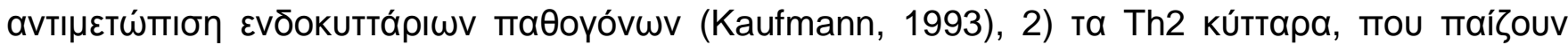

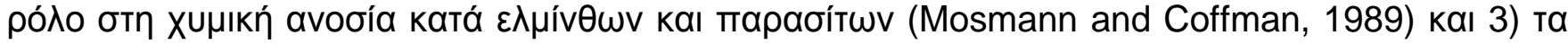

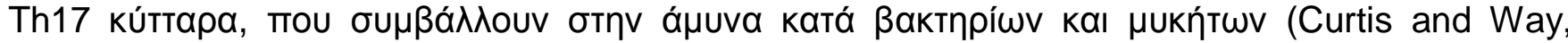

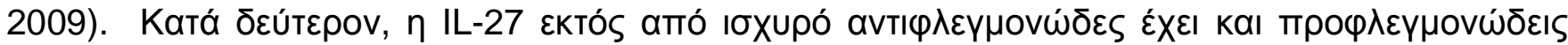

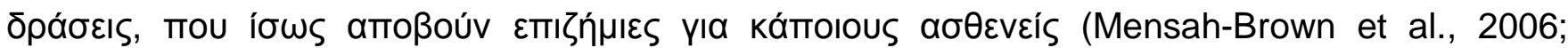
Villarino et al., 2008; Wang et al., 2008).

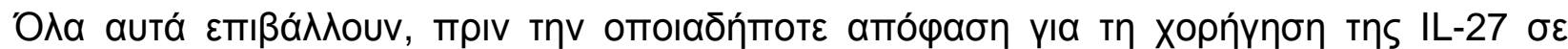

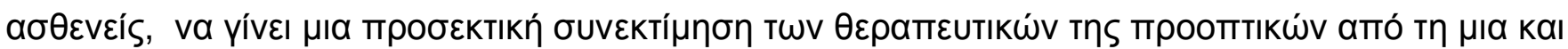

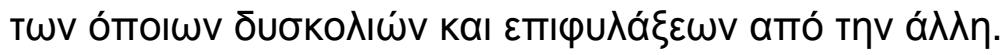


6. Bıß入ıоүрачía 
Akira, S., Uematsu, S., and Takeuchi, O. (2006). Pathogen recognition and innate immunity. Cell 124, 783801.

Amadi-Obi, A., Yu, C. R., Liu, X., Mahdi, R. M., Clarke, G. L., Nussenblatt, R. B., Gery, I., Lee, Y. S., and Egwuagu, C. E. (2007). TH17 cells contribute to uveitis and scleritis and are expanded by IL-2 and inhibited by IL-27/STAT1. Nat Med 13, 711-718.

Anderson, D. M., Maraskovsky, E., Billingsley, W. L., Dougall, W. C., Tometsko, M. E., Roux, E. R., Teepe, M. C., DuBose, R. F., Cosman, D., and Galibert, L. (1997). A homologue of the TNF receptor and its ligand enhance T-cell growth and dendritic-cell function. Nature 390, 175-179.

Andreakos, E., Sacre, S., Foxwell, B. M., and Feldmann, M. (2005). The toll-like receptor-nuclear factor kappaB pathway in rheumatoid arthritis. Front Biosci 10, 2478-2488.

Annunziato, F., Cosmi, L., Liotta, F., Maggi, E., and Romagnani, S. (2009). Type 17 T helper cells-origins, features and possible roles in rheumatic disease. Nat Rev Rheumatol 5, 325-331.

Asagiri, M., Sato, K., Usami, T., Ochi, S., Nishina, H., Yoshida, H., Morita, I., Wagner, E. F., Mak, T. W., Serfling, E., and Takayanagi, H. (2005). Autoamplification of NFATc1 expression determines its essential role in bone homeostasis. J Exp Med 202, 1261-1269.

Asquith, D. L., Miller, A. M., McInnes, I. B., and Liew, F. Y. (2009). Animal models of rheumatoid arthritis. Eur J Immunol 39, 2040-2044.

Athanasou, N. A., Quinn, J., Heryet, A., Puddle, B., Woods, C. G., and McGee, J. O. (1988). The immunohistology of synovial lining cells in normal and inflamed synovium. J Pathol 155, 133-142.

Awasthi, A., Carrier, Y., Peron, J. P., Bettelli, E., Kamanaka, M., Flavell, R. A., Kuchroo, V. K., Oukka, M., and Weiner, H. L. (2007). A dominant function for interleukin 27 in generating interleukin 10producing anti-inflammatory T cells. Nat Immunol 8, 1380-1389.

Banchereau, J., and Pascual, V. (2006). Type I interferon in systemic lupus erythematosus and other autoimmune diseases. Immunity 25, 383-392.

Barton, A., Thomson, W., Ke, X., Eyre, S., Hinks, A., Bowes, J., Plant, D., Gibbons, L. J., Wilson, A. G., Bax, D. E., et al. (2008). Rheumatoid arthritis susceptibility loci at chromosomes 10p15, 12q13 and 22q13. Nat Genet 40, 1156-1159.

Barton, G. M., and Medzhitov, R. (2003). Toll-like receptor signaling pathways. Science 300, 1524-1525. Batten, M., and Ghilardi, N. (2007). The biology and therapeutic potential of interleukin 27. J Mol Med 85, 661-672. 
Batten, M., Li, J., Yi, S., Kljavin, N. M., Danilenko, D. M., Lucas, S., Lee, J., de Sauvage, F. J., and Ghilardi, N. (2006). Interleukin 27 limits autoimmune encephalomyelitis by suppressing the development of interleukin 17-producing T cells. Nat Immunol 7, 929-936.

Beebe, A. M., Cua, D. J., and de Waal Malefyt, R. (2002). The role of interleukin-10 in autoimmune disease: systemic lupus erythematosus (SLE) and multiple sclerosis (MS). Cytokine Growth Factor Rev 13, 403-412.

Begovich, A. B., Carlton, V. E., Honigberg, L. A., Schrodi, S. J., Chokkalingam, A. P., Alexander, H. C., Ardlie, K. G., Huang, Q., Smith, A. M., Spoerke, J. M., et al. (2004). A missense single-nucleotide polymorphism in a gene encoding a protein tyrosine phosphatase (PTPN22) is associated with rheumatoid arthritis. Am J Hum Genet 75, 330-337.

Bendixen, A. C., Shevde, N. K., Dienger, K. M., Willson, T. M., Funk, C. D., and Pike, J. W. (2001). IL-4 inhibits osteoclast formation through a direct action on osteoclast precursors via peroxisome proliferatoractivated receptor gamma 1. Proc Natl Acad Sci U S A 98, 2443-2448.

Berenbaum, F. (2008). New horizons and perspectives in the treatment of osteoarthritis. Arthritis Res Ther 10 Suppl 2, S1.

Boulanger, M. J., and Garcia, K. C. (2004). Shared cytokine signaling receptors: structural insights from the gp130 system. Adv Protein Chem 68, 107-146.

Bowes, J., and Barton, A. (2008). Recent advances in the genetics of RA susceptibility. Rheumatology (Oxford) 47, 399-402.

Boyle, W. J., Simonet, W. S., and Lacey, D. L. (2003). Osteoclast differentiation and activation. Nature 423, 337-342.

Bravo, J., and Heath, J. K. (2000). Receptor recognition by gp130 cytokines. Embo J 19, 2399-2411.

Brennan, F. M., Chantry, D., Jackson, A., Maini, R., and Feldmann, M. (1989a). Inhibitory effect of TNF alpha antibodies on synovial cell interleukin-1 production in rheumatoid arthritis. Lancet 2, 244-247.

Brennan, F. M., Chantry, D., Jackson, A. M., Maini, R. N., and Feldmann, M. (1989b). Cytokine production in culture by cells isolated from the synovial membrane. J Autoimmun 2 Suppl, 177-186.

Brennan, F. M., and McInnes, I. B. (2008). Evidence that cytokines play a role in rheumatoid arthritis. J Clin Invest 118, 3537-3545.

Brent, L. H. (2009). Inflammatory arthritis: an overview for primary care physicians. Postgrad Med 121, 148-162.

Brentano, F., Kyburz, D., and Gay, S. (2009). Toll-like receptors and rheumatoid arthritis. Methods Mol Biol 517, 329-343. 
Brentano, F., Kyburz, D., Schorr, O., Gay, R., and Gay, S. (2005). The role of Toll-like receptor signalling in the pathogenesis of arthritis. Cell Immunol 233, 90-96.

Bresnihan, B., Tak, P. P., Emery, P., Klareskog, L., and Breedveld, F. (2000). Synovial biopsy in arthritis research: five years of concerted European collaboration. Ann Rheum Dis 59, 506-511.

Brown, K. D., Claudio, E., and Siebenlist, U. (2008). The roles of the classical and alternative nuclear factor-kappaB pathways: potential implications for autoimmunity and rheumatoid arthritis. Arthritis Res Ther 10, 212.

Burger, D., Dayer, J. M., Palmer, G., and Gabay, C. (2006). Is IL-1 a good therapeutic target in the treatment of arthritis? Best Pract Res Clin Rheumatol 20, 879-896.

Cao, Y., Doodes, P. D., Glant, T. T., and Finnegan, A. (2008). IL-27 induces a Th1 immune response and susceptibility to experimental arthritis. J Immunol 180, 922-930.

Carbia-Nagashima, A., and Arzt, E. (2004). Intracellular proteins and mechanisms involved in the control of gp130/JAK/STAT cytokine signaling. IUBMB Life 56, 83-88.

Carl, J. W., and Bai, X. F. (2008). IL27: Its Roles in the Induction and Inhibition of Inflammation. Int J Clin Exp Pathol 1, 117-123.

Cella, M., Buonsanti, C., Strader, C., Kondo, T., Salmaggi, A., and Colonna, M. (2003). Impaired differentiation of osteoclasts in TREM-2-deficient individuals. J Exp Med 198, 645-651.

Chen, L., Wei, X. Q., Evans, B., Jiang, W., and Aeschlimann, D. (2008). IL-23 promotes osteoclast formation by up-regulation of receptor activator of NF-kappaB (RANK) expression in myeloid precursor cells. Eur J Immunol 38, 2845-2854.

Chen, Q., Ghilardi, N., Wang, H., Baker, T., Xie, M. H., Gurney, A., Grewal, I. S., and de Sauvage, F. J. (2000). Development of Th1-type immune responses requires the type I cytokine receptor TCCR. Nature 407, 916-920.

Chiu, Y. H., Zhao, M., and Chen, Z. J. (2009). Ubiquitin in NF-kappaB signaling. Chem Rev 109, 15491560 .

Chow, D. C., Brevnova, L., He, X. L., Martick, M. M., Bankovich, A., and Garcia, K. C. (2002). A structural template for gp130-cytokine signaling assemblies. Biochim Biophys Acta 1592, 225-235.

Choy, E. H., and Panayi, G. S. (2001). Cytokine pathways and joint inflammation in rheumatoid arthritis. N Engl J Med 344, 907-916.

Chung, S. A., and Criswell, L. A. (2007). PTPN22: its role in SLE and autoimmunity. Autoimmunity 40, 582-590. 
Clark, W., Jobanputra, P., Barton, P., and Burls, A. (2004). The clinical and cost-effectiveness of anakinra for the treatment of rheumatoid arthritis in adults: a systematic review and economic analysis. Health Technol Assess 8, iii-iv, ix-x, 1-105.

Coenen, M. J., and Gregersen, P. K. (2009). Rheumatoid arthritis: a view of the current genetic landscape. Genes Immun 10, 101-111.

Coffman, R. L. (2006). Origins of the T(H)1-T(H)2 model: a personal perspective. Nat Immunol 7, 539541.

Coffman, R. L., and Carty, J. (1986). A T cell activity that enhances polyclonal IgE production and its inhibition by interferon-gamma. J Immunol 136, 949-954.

Cohen, S. B., Dore, R. K., Lane, N. E., Ory, P. A., Peterfy, C. G., Sharp, J. T., van der Heijde, D., Zhou, L., Tsuji, W., and Newmark, R. (2008). Denosumab treatment effects on structural damage, bone mineral density, and bone turnover in rheumatoid arthritis: a twelve-month, multicenter, randomized, double-blind, placebo-controlled, phase II clinical trial. Arthritis Rheum 58, 1299-1309.

Colgan, J., and Rothman, P. (2006). All in the family: IL-27 suppression of T(H)-17 cells. Nat Immunol 7, 899-901.

Collison, L. W., and Vignali, D. A. (2008). Interleukin-35: odd one out or part of the family? Immunol Rev $226,248-262$.

Collison, L. W., Workman, C. J., Kuo, T. T., Boyd, K., Wang, Y., Vignali, K. M., Cross, R., Sehy, D., Blumberg, R. S., and Vignali, D. A. (2007). The inhibitory cytokine IL-35 contributes to regulatory T-cell function. Nature 450, 566-569.

Colonna, M., Turnbull, I., and Klesney-Tait, J. (2007). The enigmatic function of TREM-2 in osteoclastogenesis. Adv Exp Med Biol 602, 97-105.

Costenbader, K. H., and Karlson, E. W. (2006). Cigarette smoking and autoimmune disease: what can we learn from epidemiology? Lupus 15, 737-745.

Coulomb-L'Hermine, A., Larousserie, F., Pflanz, S., Bardel, E., Kastelein, R. A., and Devergne, O. (2007). Expression of interleukin-27 by human trophoblast cells. Placenta 28, 1133-1140.

Curtis, M. M., and Way, S. S. (2009). Interleukin-17 in host defence against bacterial, mycobacterial and fungal pathogens. Immunology 126, 177-185.

Daoussis, D., Andonopoulos, A. P., and Liossis, S. N. (2008). Wnt Pathway and IL-17: Novel Regulators of Joint Remodeling in Rheumatic Diseases. Looking Beyond the RANK-RANKL-OPG Axis. Semin Arthritis Rheum.

Darnell, J. E., Jr. (1997). STATs and gene regulation. Science 277, 1630-1635. 
Darnell, J. E., Jr., Kerr, I. M., and Stark, G. R. (1994). Jak-STAT pathways and transcriptional activation in response to IFNs and other extracellular signaling proteins. Science 264, 1415-1421.

Davidson, A., and Diamond, B. (2001). Autoimmune diseases. N Engl J Med 345, 340-350.

Day, C. J., Kim, M. S., Lopez, C. M., Nicholson, G. C., and Morrison, N. A. (2005). NFAT expression in human osteoclasts. J Cell Biochem 95, 17-23.

Dayer, J. M. (2002). Evidence for the biological modulation of IL-1 activity: the role of IL-1Ra. Clin Exp Rheumatol 20, S14-20.

Dayer, J. M. (2003). The pivotal role of interleukin-1 in the clinical manifestations of rheumatoid arthritis. Rheumatology (Oxford) 42 Suppl 2, ii3-10.

de Vries, R. R., Huizinga, T. W., and Toes, R. E. (2006). HLA and RA revisited: citrullinated food for the SE hypothesis, the DR6 effect, and NIMA. Hum Immunol 67, 454-459.

Devergne, O., Hummel, M., Koeppen, H., Le Beau, M. M., Nathanson, E. C., Kieff, E., and Birkenbach, M. (1996). A novel interleukin-12 p40-related protein induced by latent Epstein-Barr virus infection in B lymphocytes. J Virol 70, 1143-1153.

Dinarello, C. A. (1996). Biologic basis for interleukin-1 in disease. Blood 87, 2095-2147.

Dinarello, C. A. (2002). The IL-1 family and inflammatory diseases. Clin Exp Rheumatol 20, S1-13.

Dinarello, C. A. (2007). Historical insights into cytokines. Eur J Immunol 37 Suppl 1, S34-45.

Dinarello, C. A. (2009). Immunological and inflammatory functions of the interleukin-1 family. Annu Rev Immunol 27, 519-550.

Diveu, C., McGeachy, M. J., Boniface, K., Stumhofer, J. S., Sathe, M., Joyce-Shaikh, B., Chen, Y., Tato, C. M., McClanahan, T. K., de Waal Malefyt, R., et al. (2009). IL-27 blocks RORc expression to inhibit lineage commitment of Th17 cells. J Immunol 182, 5748-5756.

Dubin, P. J., and Kolls, J. K. (2008). Th17 cytokines and mucosal immunity. Immunol Rev 226, 160-171.

El Kasmi, K. C., Holst, J., Coffre, M., Mielke, L., de Pauw, A., Lhocine, N., Smith, A. M., Rutschman, R., Kaushal, D., Shen, Y., et al. (2006). General nature of the STAT3-activated anti-inflammatory response. J Immunol 177, 7880-7888.

Elliott, M. J., Maini, R. N., Feldmann, M., Kalden, J. R., Antoni, C., Smolen, J. S., Leeb, B., Breedveld, F. C., Macfarlane, J. D., Bijl, H., and et al. (1994). Randomised double-blind comparison of chimeric monoclonal antibody to tumour necrosis factor alpha (cA2) versus placebo in rheumatoid arthritis. Lancet $344,1105-1110$. 
Elliott, M. J., Maini, R. N., Feldmann, M., Long-Fox, A., Charles, P., Katsikis, P., Brennan, F. M., Walker, J., Bijl, H., Ghrayeb, J., and et al. (1993). Treatment of rheumatoid arthritis with chimeric monoclonal antibodies to tumor necrosis factor alpha. Arthritis Rheum 36, 1681-1690.

Ernst, M., and Jenkins, B. J. (2004). Acquiring signalling specificity from the cytokine receptor gp130. Trends Genet 20, 23-32.

Evans, K. E., and Fox, S. W. (2007). Interleukin-10 inhibits osteoclastogenesis by reducing NFATc1 expression and preventing its translocation to the nucleus. BMC Cell Biol 8, 4.

Feldmann, M. (2008). Many cytokines are very useful therapeutic targets in disease. J Clin Invest 118 , 3533-3536.

Feldmann, M. (2009). Translating molecular insights in autoimmunity into effective therapy. Annu Rev Immunol 27, 1-27.

Feldmann, M., Brennan, F. M., Foxwell, B. M., and Maini, R. N. (2001). The role of TNF alpha and IL-1 in rheumatoid arthritis. Curr Dir Autoimmun 3, 188-199.

Feldmann, M., and Maini, R. N. (2002). Discovery of TNF-alpha as a therapeutic target in rheumatoid arthritis: preclinical and clinical studies. Joint Bone Spine 69, 12-18.

Feldmann, M., and Maini, S. R. (2008). Role of cytokines in rheumatoid arthritis: an education in pathophysiology and therapeutics. Immunol Rev 223, 7-19.

Feng, X. M., Liu, N., Yang, S. G., Hu, L. Y., Chen, X. L., Fang, Z. H., Ren, Q., Lu, S. H., Liu, B., and Han, Z. C. (2008). Regulation of the class II and class I MHC pathways in human THP-1 monocytic cells by interleukin-27. Biochem Biophys Res Commun 367, 553-559.

Fernando, M. M., Stevens, C. R., Walsh, E. C., De Jager, P. L., Goyette, P., Plenge, R. M., Vyse, T. J., and Rioux, J. D. (2008). Defining the role of the MHC in autoimmunity: a review and pooled analysis. PLoS Genet 4, e1000024.

Firestein, G. S. (2003). Evolving concepts of rheumatoid arthritis. Nature 423, 356-361.

Firestein, G. S. (2007). Biomedicine. Every joint has a silver lining. Science 315, 952-953.

Firestein, G. S. (2009). Rheumatoid arthritis in a mouse? Nat Clin Pract Rheumatol 5, 1.

Firestein, G. S., Alvaro-Gracia, J. M., and Maki, R. (1990). Quantitative analysis of cytokine gene expression in rheumatoid arthritis. J Immunol 144, 3347-3353.

Firestein, G. S., and Zvaifler, N. J. (1987). Peripheral blood and synovial fluid monocyte activation in inflammatory arthritis. II. Low levels of synovial fluid and synovial tissue interferon suggest that gammainterferon is not the primary macrophage activating factor. Arthritis Rheum 30, 864-871. 
Firestein, G. S., and Zvaifler, N. J. (2002). How important are T cells in chronic rheumatoid synovitis?: II. T cell-independent mechanisms from beginning to end. Arthritis Rheum 46, 298-308.

Fischer, P., Lehmann, U., Sobota, R. M., Schmitz, J., Niemand, C., Linnemann, S., Haan, S., Behrmann, I., Yoshimura, A., Johnston, J. A., et al. (2004). The role of the inhibitors of interleukin-6 signal transduction SHP2 and SOCS3 for desensitization of interleukin-6 signalling. Biochem J 378, 449-460.

Fitzgerald, D. C., Ciric, B., Touil, T., Harle, H., Grammatikopolou, J., Das Sarma, J., Gran, B., Zhang, G. X., and Rostami, A. (2007a). Suppressive effect of IL-27 on encephalitogenic Th17 cells and the effector phase of experimental autoimmune encephalomyelitis. J Immunol 179, 3268-3275.

Fitzgerald, D. C., Zhang, G. X., El-Behi, M., Fonseca-Kelly, Z., Li, H., Yu, S., Saris, C. J., Gran, B., Ciric, B., and Rostami, A. (2007b). Suppression of autoimmune inflammation of the central nervous system by interleukin 10 secreted by interleukin 27-stimulated T cells. Nat Immunol 8, 1372-1379.

Fukada, T., Yoshida, Y., Nishida, K., Ohtani, T., Shirogane, T., Hibi, M., and Hirano, T. (1999). Signaling through Gp130: toward a general scenario of cytokine action. Growth Factors 17, 81-91.

Furuzawa-Carballeda, J., Vargas-Rojas, M. I., and Cabral, A. R. (2007). Autoimmune inflammation from the Th17 perspective. Autoimmun Rev 6, 169-175.

Gabay, C., and McInnes, I. B. (2009). The biological and clinical importance of the 'new generation' cytokines in rheumatic diseases. Arthritis Res Ther 11, 230.

Gabriel, S. E. (2001). The epidemiology of rheumatoid arthritis. Rheum Dis Clin North Am 27, 269-281.

Gaffen, S. L. (2009). Structure and signalling in the IL-17 receptor family. Nat Rev Immunol 9, 556-567.

Galibert, L., Tometsko, M. E., Anderson, D. M., Cosman, D., and Dougall, W. C. (1998). The involvement of multiple tumor necrosis factor receptor (TNFR)-associated factors in the signaling mechanisms of receptor activator of NF-kappaB, a member of the TNFR superfamily. J Biol Chem 273, 34120-34127.

Gee, K., Guzzo, C., Che Mat, N. F., Ma, W., and Kumar, A. (2009). The IL-12 family of cytokines in infection, inflammation and autoimmune disorders. Inflamm Allergy Drug Targets 8, 40-52.

Gohda, J., Akiyama, T., Koga, T., Takayanagi, H., Tanaka, S., and Inoue, J. (2005). RANK-mediated amplification of TRAF6 signaling leads to NFATc1 induction during osteoclastogenesis. Embo J 24, 790799.

Goldberg, R., Wildbaum, G., Zohar, Y., Maor, G., and Karin, N. (2004). Suppression of ongoing adjuvantinduced arthritis by neutralizing the function of the p28 subunit of IL-27. J Immunol 173, 1171-1178.

Goldring, M. B., and Goldring, S. R. (2007). Osteoarthritis. J Cell Physiol 213, 626-634.

Goriely, S., Neurath, M. F., and Goldman, M. (2008). How microorganisms tip the balance between interleukin-12 family members. Nat Rev Immunol 8, 81-86. 
Gorman, J. D., and Criswell, L. A. (2002). The shared epitope and severity of rheumatoid arthritis. Rheum Dis Clin North Am 28, 59-78.

Grassi, W., De Angelis, R., Lamanna, G., and Cervini, C. (1998). The clinical features of rheumatoid arthritis. Eur J Radiol 27 Suppl 1, S18-24.

Graudal, N. (2004). The natural history and prognosis of rheumatoid arthritis: association of radiographic outcome with process variables, joint motion and immune proteins. Scand J Rheumatol Suppl, 1-38.

Gravallese, E. M., Harada, Y., Wang, J. T., Gorn, A. H., Thornhill, T. S., and Goldring, S. R. (1998). Identification of cell types responsible for bone resorption in rheumatoid arthritis and juvenile rheumatoid arthritis. Am J Pathol 152, 943-951.

Gravallese, E. M., Manning, C., Tsay, A., Naito, A., Pan, C., Amento, E., and Goldring, S. R. (2000). Synovial tissue in rheumatoid arthritis is a source of osteoclast differentiation factor. Arthritis Rheum 43, 250-258.

Gregersen, P. K., and Olsson, L. M. (2009). Recent advances in the genetics of autoimmune disease. Annu Rev Immunol 27, 363-391.

Haddad, J. J. (2002). Cytokines and related receptor-mediated signaling pathways. Biochem Biophys Res Commun 297, 700-713.

Halverson, P. B. (1995). Extraarticular manifestations of rheumatoid arthritis. Orthop Nurs 14, 47-50.

Hamilton, J. A. (1983). Hypothesis: in vitro evidence for the invasive and tumor-like properties of the rheumatoid pannus. J Rheumatol 10, 845-851.

Harel, M., and Shoenfeld, Y. (2006). Predicting and preventing autoimmunity, myth or reality? Ann N Y Acad Sci 1069, 322-345.

Haworth, C., Brennan, F. M., Chantry, D., Turner, M., Maini, R. N., and Feldmann, M. (1991). Expression of granulocyte-macrophage colony-stimulating factor in rheumatoid arthritis: regulation by tumor necrosis factor-alpha. Eur J Immunol 21, 2575-2579.

Heinrich, P. C., Behrmann, I., Muller-Newen, G., Schaper, F., and Graeve, L. (1998). Interleukin-6-type cytokine signalling through the gp130/Jak/STAT pathway. Biochem J 334 ( Pt 2), 297-314.

Helming, L., and Gordon, S. (2007). The molecular basis of macrophage fusion. Immunobiology 212, $785-$ 793.

Herrero, C., Hu, X., Li, W. P., Samuels, S., Sharif, M. N., Kotenko, S., and Ivashkiv, L. B. (2003). Reprogramming of IL-10 activity and signaling by IFN-gamma. J Immunol 171, 5034-5041. 
Hibbert, L., Pflanz, S., De Waal Malefyt, R., and Kastelein, R. A. (2003). IL-27 and IFN-alpha signal via Stat 1 and Stat3 and induce T-Bet and IL-12Rbeta2 in naive T cells. J Interferon Cytokine Res 23, 513-522. Hideshima, T., Podar, K., Chauhan, D., and Anderson, K. C. (2005). Cytokines and signal transduction. Best Pract Res Clin Haematol 18, 509-524.

Hitchon, C. A., and el-Gabalawy, H. S. (2003). The histopathology of early synovitis. Clin Exp Rheumatol 21, S28-36.

Ho, H. H., and Ivashkiv, L. B. (2006). Role of STAT3 in type I interferon responses. Negative regulation of STAT1-dependent inflammatory gene activation. J Biol Chem 281, 14111-14118.

Hogan, P. G., Chen, L., Nardone, J., and Rao, A. (2003). Transcriptional regulation by calcium, calcineurin, and NFAT. Genes Dev 17, 2205-2232.

Hu, X., Chakravarty, S. D., and Ivashkiv, L. B. (2008a). Regulation of interferon and Toll-like receptor signaling during macrophage activation by opposing feedforward and feedback inhibition mechanisms. Immunol Rev 226, 41-56.

Hu, X., Chung, A. Y., Wu, I., Foldi, J., Chen, J., Ji, J. D., Tateya, T., Kang, Y. J., Han, J., Gessler, M., et al. (2008b). Integrated regulation of Toll-like receptor responses by Notch and interferon-gamma pathways. Immunity 29, 691-703.

Hu, X., Herrero, C., Li, W. P., Antoniv, T. T., Falck-Pedersen, E., Koch, A. E., Woods, J. M., Haines, G. K., and Ivashkiv, L. B. (2002). Sensitization of IFN-gamma Jak-STAT signaling during macrophage activation. Nat Immunol 3, 859-866.

Hu, X., Ho, H. H., Lou, O., Hidaka, C., and Ivashkiv, L. B. (2005). Homeostatic role of interferons conferred by inhibition of IL-1-mediated inflammation and tissue destruction. J Immunol 175, 131-138.

Hu, X., Paik, P. K., Chen, J., Yarilina, A., Kockeritz, L., Lu, T. T., Woodgett, J. R., and Ivashkiv, L. B. (2006). IFN-gamma suppresses IL-10 production and synergizes with TLR2 by regulating GSK3 and CREB/AP-1 proteins. Immunity 24, 563-574.

Huang, Q., Ma, Y., Adebayo, A., and Pope, R. M. (2007). Increased macrophage activation mediated through toll-like receptors in rheumatoid arthritis. Arthritis Rheum 56, 2192-2201.

Huang, Q. Q., Sobkoviak, R., Jockheck-Clark, A. R., Shi, B., Mandelin, A. M., 2nd, Tak, P. P., Haines, G. K., 3rd, Nicchitta, C. V., and Pope, R. M. (2009). Heat shock protein 96 is elevated in rheumatoid arthritis and activates macrophages primarily via TLR2 signaling. J Immunol 182, 4965-4973.

Huber, M., Steinwald, V., Guralnik, A., Brustle, A., Kleemann, P., Rosenplanter, C., Decker, T., and Lohoff, M. (2008). IL-27 inhibits the development of regulatory T cells via STAT3. Int Immunol 20, 223234. 
Humphrey, M. B., Daws, M. R., Spusta, S. C., Niemi, E. C., Torchia, J. A., Lanier, L. L., Seaman, W. E., and Nakamura, M. C. (2006). TREM2, a DAP12-associated receptor, regulates osteoclast differentiation and function. J Bone Miner Res 21, 237-245.

Hunter, C. A. (2005). New IL-12-family members: IL-23 and IL-27, cytokines with divergent functions. Nat Rev Immunol 5, 521-531.

Hunter, C. A., Villarino, A., Artis, D., and Scott, P. (2004). The role of IL-27 in the development of T-cell responses during parasitic infections. Immunol Rev 202, 106-114.

Ikeda, F., Nishimura, R., Matsubara, T., Tanaka, S., Inoue, J., Reddy, S. V., Hata, K., Yamashita, K., Hiraga, T., Watanabe, T., et al. (2004). Critical roles of c-Jun signaling in regulation of NFAT family and RANKL-regulated osteoclast differentiation. J Clin Invest 114, 475-484.

Ishida, N., Hayashi, K., Hoshijima, M., Ogawa, T., Koga, S., Miyatake, Y., Kumegawa, M., Kimura, T., and Takeya, T. (2002). Large scale gene expression analysis of osteoclastogenesis in vitro and elucidation of NFAT2 as a key regulator. J Biol Chem 277, 41147-41156.

Ishii, M., Egen, J. G., Klauschen, F., Meier-Schellersheim, M., Saeki, Y., Vacher, J., Proia, R. L., and Germain, R. N. (2009). Sphingosine-1-phosphate mobilizes osteoclast precursors and regulates bone homeostasis. Nature 458, 524-528.

Ivanov, II, Zhou, L., and Littman, D. R. (2007). Transcriptional regulation of Th17 cell differentiation. Semin Immunol 19, 409-417.

Ivashkiv, L. B. (2009). Cross-regulation of signaling by ITAM-associated receptors. Nat Immunol 10, 340347.

Ivashkiv, L. B., and $\mathrm{Hu}, \mathrm{X}$. (2003). The JAK/STAT pathway in rheumatoid arthritis: pathogenic or protective? Arthritis Rheum 48, 2092-2096.

Ivashkiv, L. B., and Hu, X. (2004). Signaling by STATs. Arthritis Res Ther 6, 159-168.

Iwanaga, T., Shikichi, M., Kitamura, H., Yanase, H., and Nozawa-Inoue, K. (2000). Morphology and functional roles of synoviocytes in the joint. Arch Histol Cytol 63, 17-31.

Janeway, C. A., Jr., and Medzhitov, R. (1998). Introduction: the role of innate immunity in the adaptive immune response. Semin Immunol 10, 349-350.

Kamimura, D., Ishihara, K., and Hirano, T. (2003). IL-6 signal transduction and its physiological roles: the signal orchestration model. Rev Physiol Biochem Pharmacol 149, 1-38.

Kamiya, S., Nakamura, C., Fukawa, T., Ono, K., Ohwaki, T., Yoshimoto, T., and Wada, S. (2007). Effects of IL-23 and IL-27 on osteoblasts and osteoclasts: inhibitory effects on osteoclast differentiation. J Bone Miner Metab 25, 277-285. 
Kamiya, S., Owaki, T., Morishima, N., Fukai, F., Mizuguchi, J., and Yoshimoto, T. (2004). An indispensable role for STAT1 in IL-27-induced T-bet expression but not proliferation of naive CD4+ T cells. J Immunol 173, 3871-3877.

Kanazawa, K., and Kudo, A. (2005). TRAF2 is essential for TNF-alpha-induced osteoclastogenesis. J Bone Miner Res 20, 840-847.

Kaplan, M. J. (2009). Management of cardiovascular disease risk in chronic inflammatory disorders. Nat Rev Rheumatol 5, 208-217.

Kastelein, R. A., Hunter, C. A., and Cua, D. J. (2007). Discovery and biology of IL-23 and IL-27: related but functionally distinct regulators of inflammation. Annu Rev Immunol 25, 221-242.

Kaufmann, S. H. (1993). Immunity to intracellular bacteria. Annu Rev Immunol 11, 129-163.

Keffer, J., Probert, L., Cazlaris, H., Georgopoulos, S., Kaslaris, E., Kioussis, D., and Kollias, G. (1991). Transgenic mice expressing human tumour necrosis factor: a predictive genetic model of arthritis. Embo J $10,4025-4031$.

Kelso, A. (2000). Cytokines and their receptors: an overview. Ther Drug Monit 22, 40-43.

Klareskog, L., Padyukov, L., Lorentzen, J., and Alfredsson, L. (2006a). Mechanisms of disease: Genetic susceptibility and environmental triggers in the development of rheumatoid arthritis. Nat Clin Pract Rheumatol 2, 425-433.

Klareskog, L., Padyukov, L., Ronnelid, J., and Alfredsson, L. (2006b). Genes, environment and immunity in the development of rheumatoid arthritis. Curr Opin Immunol 18, 650-655.

Kobayashi, K., Takahashi, N., Jimi, E., Udagawa, N., Takami, M., Kotake, S., Nakagawa, N., Kinosaki, M., Yamaguchi, K., Shima, N., et al. (2000). Tumor necrosis factor alpha stimulates osteoclast differentiation by a mechanism independent of the ODF/RANKL-RANK interaction. J Exp Med 191, 275286.

Kobayashi, N., Kadono, Y., Naito, A., Matsumoto, K., Yamamoto, T., Tanaka, S., and Inoue, J. (2001). Segregation of TRAF6-mediated signaling pathways clarifies its role in osteoclastogenesis. Embo J 20, 1271-1280.

Kobayashi, S., Ikari, K., Kaneko, H., Kochi, Y., Yamamoto, K., Shimane, K., Nakamura, Y., Toyama, Y., Mochizuki, T., Tsukahara, S., et al. (2008a). Association of STAT4 with susceptibility to rheumatoid arthritis and systemic lupus erythematosus in the Japanese population. Arthritis Rheum 58, 1940-1946.

Kobayashi, S., Momohara, S., Kamatani, N., and Okamoto, H. (2008b). Molecular aspects of rheumatoid arthritis: role of environmental factors. Febs J 275, 4456-4462.

Kochi, Y., Suzuki, A., Yamada, R., and Yamamoto, K. (2009). Genetics of rheumatoid arthritis: underlying evidence of ethnic differences. J Autoimmun 32, 158-162. 
Koga, T., Inui, M., Inoue, K., Kim, S., Suematsu, A., Kobayashi, E., Iwata, T., Ohnishi, H., Matozaki, T., Kodama, T., et al. (2004). Costimulatory signals mediated by the ITAM motif cooperate with RANKL for bone homeostasis. Nature 428, 758-763.

Koga, T., Matsui, Y., Asagiri, M., Kodama, T., de Crombrugghe, B., Nakashima, K., and Takayanagi, H. (2005). NFAT and Osterix cooperatively regulate bone formation. Nat Med 11, 880-885.

Kolls, J. K., and Linden, A. (2004). Interleukin-17 family members and inflammation. Immunity 21, 467476.

Kruithof, E., Baeten, D., De Rycke, L., Vandooren, B., Foell, D., Roth, J., Canete, J. D., Boots, A. M., Veys, E. M., and De Keyser, F. (2005). Synovial histopathology of psoriatic arthritis, both oligo- and polyarticular, resembles spondyloarthropathy more than it does rheumatoid arthritis. Arthritis Res Ther 7, R569-580.

Kumar, H., Kawai, T., and Akira, S. (2009). Toll-like receptors and innate immunity. Biochem Biophys Res Commun 388, 621-625.

Kurth, I., Horsten, U., Pflanz, S., Timmermann, A., Kuster, A., Dahmen, H., Tacken, I., Heinrich, P. C., and Muller-Newen, G. (2000). Importance of the membrane-proximal extracellular domains for activation of the signal transducer glycoprotein 130. J Immunol 164, 273-282.

Lacey, D. L., Timms, E., Tan, H. L., Kelley, M. J., Dunstan, C. R., Burgess, T., Elliott, R., Colombero, A., Elliott, G., Scully, S., et al. (1998). Osteoprotegerin ligand is a cytokine that regulates osteoclast differentiation and activation. Cell 93, 165-176.

Lang, R. (2005). Tuning of macrophage responses by Stat3-inducing cytokines: molecular mechanisms and consequences in infection. Immunobiology 210, 63-76.

Larousserie, F., Pflanz, S., Coulomb-L'Hermine, A., Brousse, N., Kastelein, R., and Devergne, O. (2004). Expression of IL-27 in human Th1-associated granulomatous diseases. J Pathol 202, 164-171.

Lee, D. M., Kiener, H. P., Agarwal, S. K., Noss, E. H., Watts, G. F., Chisaka, O., Takeichi, M., and Brenner, M. B. (2007). Cadherin-11 in synovial lining formation and pathology in arthritis. Science 315, 1006-1010.

Lee, S. K., Goldring, S. R., and Lorenzo, J. A. (1995). Expression of the calcitonin receptor in bone marrow cell cultures and in bone: a specific marker of the differentiated osteoclast that is regulated by calcitonin. Endocrinology 136, 4572-4581.

Leibbrandt, A., and Penninger, J. M. (2009). RANKL/RANK as key factors for osteoclast development and bone loss in arthropathies. Adv Exp Med Biol 649, 100-113.

Levy, D. E., and Darnell, J. E., Jr. (2002). Stats: transcriptional control and biological impact. Nat Rev Mol Cell Biol 3, 651-662. 
Ling, P., Gately, M. K., Gubler, U., Stern, A. S., Lin, P., Hollfelder, K., Su, C., Pan, Y. C., and Hakimi, J. (1995). Human IL-12 p40 homodimer binds to the IL-12 receptor but does not mediate biologic activity. J Immunol 154, 116-127.

Linn-Rasker, S. P., van der Helm-van Mil, A. H., van Gaalen, F. A., Kloppenburg, M., de Vries, R. R., le Cessie, S., Breedveld, F. C., Toes, R. E., and Huizinga, T. W. (2006). Smoking is a risk factor for anti-CCP antibodies only in rheumatoid arthritis patients who carry HLA-DRB1 shared epitope alleles. Ann Rheum Dis $65,366-371$.

Lipsky, P. E. (2007). Why does rheumatoid arthritis involve the joints? N Engl J Med 356, 2419-2420.

Lipsky, P. E., van der Heijde, D. M., St Clair, E. W., Furst, D. E., Breedveld, F. C., Kalden, J. R., Smolen, J. S., Weisman, M., Emery, P., Feldmann, M., et al. (2000). Infliximab and methotrexate in the treatment of rheumatoid arthritis. Anti-Tumor Necrosis Factor Trial in Rheumatoid Arthritis with Concomitant Therapy Study Group. N Engl J Med 343, 1594-1602.

Liu, J., Guan, X., and Ma, X. (2007). Regulation of IL-27 p28 gene expression in macrophages through MyD88- and interferon-gamma-mediated pathways. J Exp Med 204, 141-152.

Lleo, A., Battezzati, P. M., Selmi, C., Gershwin, M. E., and Podda, M. (2008). Is autoimmunity a matter of sex? Autoimmun Rev 7, 626-630.

Lohr, J., Knoechel, B., and Abbas, A. K. (2006). Regulatory T cells in the periphery. Immunol Rev 212, 149-162.

Lomaga, M. A., Yeh, W. C., Sarosi, I., Duncan, G. S., Furlonger, C., Ho, A., Morony, S., Capparelli, C., Van, G., Kaufman, S., et al. (1999). TRAF6 deficiency results in osteopetrosis and defective interleukin-1, CD40, and LPS signaling. Genes Dev 13, 1015-1024.

Lucas, S., Ghilardi, N., Li, J., and de Sauvage, F. J. (2003). IL-27 regulates IL-12 responsiveness of naive CD4+ T cells through Stat1-dependent and -independent mechanisms. Proc Natl Acad Sci U S A 100, 15047-15052.

Maini, R., St Clair, E. W., Breedveld, F., Furst, D., Kalden, J., Weisman, M., Smolen, J., Emery, P., Harriman, G., Feldmann, M., and Lipsky, P. (1999). Infliximab (chimeric anti-tumour necrosis factor alpha monoclonal antibody) versus placebo in rheumatoid arthritis patients receiving concomitant methotrexate: a randomised phase III trial. ATTRACT Study Group. Lancet 354, 1932-1939.

Marino, C., and McDonald, E. (1991). Osteoarthritis and rheumatoid arthritis in elderly patients. Differentiation and treatment. Postgrad Med 90, 237-243.

Matsuo, K., and Irie, N. (2008). Osteoclast-osteoblast communication. Arch Biochem Biophys 473, 201209. 
McInnes, I. B., and Schett, G. (2007). Cytokines in the pathogenesis of rheumatoid arthritis. Nat Rev Immunol 7, 429-442.

McKellar, G. E., McCarey, D. W., Sattar, N., and McInnes, I. B. (2009). Role for TNF in atherosclerosis? Lessons from autoimmune disease. Nat Rev Cardiol 6, 410-417.

Medzhitov, R., and Janeway, C. A., Jr. (1997). Innate immunity: the virtues of a nonclonal system of recognition. Cell 91, 295-298.

Medzhitov, R., and Janeway, C. A., Jr. (1998a). An ancient system of host defense. Curr Opin Immunol 10, $12-15$.

Medzhitov, R., and Janeway, C. A., Jr. (1998b). Innate immune recognition and control of adaptive immune responses. Semin Immunol 10, 351-353.

Medzhitov, R., and Janeway, C. A., Jr. (2002). Decoding the patterns of self and nonself by the innate immune system. Science 296, 298-300.

Mensah-Brown, E. P., Shahin, A., Al-Shamsi, M., and Lukic, M. L. (2006). New members of the interleukin-12 family of cytokines: IL-23 and IL-27 modulate autoimmune diabetes. Ann N Y Acad Sci $1079,157-160$.

Mertens, M., and Singh, J. A. (2009). Anakinra for rheumatoid arthritis: a systematic review. J Rheumatol $36,1118-1125$.

Mills, K. H. (2004). Regulatory T cells: friend or foe in immunity to infection? Nat Rev Immunol 4, 841855.

Miossec, P., Korn, T., and Kuchroo, V. K. (2009). Interleukin-17 and type 17 helper T cells. N Engl J Med 361, 888-898.

Mizukami, J., Takaesu, G., Akatsuka, H., Sakurai, H., Ninomiya-Tsuji, J., Matsumoto, K., and Sakurai, N. (2002). Receptor activator of NF-kappaB ligand (RANKL) activates TAK1 mitogen-activated protein kinase kinase kinase through a signaling complex containing RANK, TAB2, and TRAF6. Mol Cell Biol 22, 992-1000.

Mjaavatten, M. D., Haugen, A. J., Helgetveit, K., Nygaard, H., Sidenvall, G., Uhlig, T., and Kvien, T. K. (2009). Pattern of joint involvement and other disease characteristics in 634 patients with arthritis of less than 16 weeks' duration. J Rheumatol 36, 1401-1406.

Molle, C., Nguyen, M., Flamand, V., Renneson, J., Trottein, F., De Wit, D., Willems, F., Goldman, M., and Goriely, S. (2007). IL-27 synthesis induced by TLR ligation critically depends on IFN regulatory factor 3. J Immunol 178, 7607-7615.

Moore, K. W., de Waal Malefyt, R., Coffman, R. L., and O'Garra, A. (2001). Interleukin-10 and the interleukin-10 receptor. Annu Rev Immunol 19, 683-765. 
Moreland, L. W., Schiff, M. H., Baumgartner, S. W., Tindall, E. A., Fleischmann, R. M., Bulpitt, K. J., Weaver, A. L., Keystone, E. C., Furst, D. E., Mease, P. J., et al. (1999). Etanercept therapy in rheumatoid arthritis. A randomized, controlled trial. Ann Intern Med 130, 478-486.

Moreno, J. L., Kaczmarek, M., Keegan, A. D., and Tondravi, M. (2003). IL-4 suppresses osteoclast development and mature osteoclast function by a STAT6-dependent mechanism: irreversible inhibition of the differentiation program activated by RANKL. Blood 102, 1078-1086.

Mosmann, T. R., Cherwinski, H., Bond, M. W., Giedlin, M. A., and Coffman, R. L. (1986). Two types of murine helper $\mathrm{T}$ cell clone. I. Definition according to profiles of lymphokine activities and secreted proteins. J Immunol 136, 2348-2357.

Mosmann, T. R., and Coffman, R. L. (1989). TH1 and TH2 cells: different patterns of lymphokine secretion lead to different functional properties. Annu Rev Immunol 7, 145-173.

Murakami, M., Kamimura, D., and Hirano, T. (2004). New IL-6 (gp130) family cytokine members, CLC/NNT1/BSF3 and IL-27. Growth Factors 22, 75-77.

Murray, P. J. (2007). The JAK-STAT signaling pathway: input and output integration. J Immunol 178, 2623-2629.

Narvaez, J. A., Narvaez, J., Serrallonga, M., De Lama, E., de Albert, M., Mast, R., and Nolla, J. M. (2008). Cervical spine involvement in rheumatoid arthritis: correlation between neurological manifestations and magnetic resonance imaging findings. Rheumatology (Oxford) 47, 1814-1819.

Negishi-Koga, T., and Takayanagi, H. (2009). Ca2+-NFATc1 signaling is an essential axis of osteoclast differentiation. Immunol Rev 231, 241-256.

Neufert, C., Becker, C., Wirtz, S., Fantini, M. C., Weigmann, B., Galle, P. R., and Neurath, M. F. (2007). IL-27 controls the development of inducible regulatory $\mathrm{T}$ cells and Th17 cells via differential effects on STAT1. Eur J Immunol 37, 1809-1816.

Niedbala, W., Cai, B., Wei, X., Patakas, A., Leung, B. P., McInnes, I. B., and Liew, F. Y. (2008). Interleukin 27 attenuates collagen-induced arthritis. Ann Rheum Dis 67, 1474-1479.

Niedbala, W., Wei, X. Q., Cai, B., Hueber, A. J., Leung, B. P., McInnes, I. B., and Liew, F. Y. (2007). IL35 is a novel cytokine with therapeutic effects against collagen-induced arthritis through the expansion of regulatory T cells and suppression of Th17 cells. Eur J Immunol 37, 3021-3029.

Nurmohamed, M. T. (2009). Cardiovascular risk in rheumatoid arthritis. Autoimmun Rev 8, 663-667.

O'Dell, J. R. (2004). Therapeutic strategies for rheumatoid arthritis. N Engl J Med 350, 2591-2602.

O'Neill, L. A. (2008). Primer: Toll-like receptor signaling pathways--what do rheumatologists need to know? Nat Clin Pract Rheumatol 4, 319-327. 
Olsen, N. J., and Stein, C. M. (2004). New drugs for rheumatoid arthritis. N Engl J Med 350, 2167-2179.

Oppenheim, J. J. (2001). Cytokines: past, present, and future. Int J Hematol 74, 3-8.

Orbach, H., and Shoenfeld, Y. (2007). Hyperprolactinemia and autoimmune diseases. Autoimmun Rev 6 , 537-542.

Owaki, T., Asakawa, M., Fukai, F., Mizuguchi, J., and Yoshimoto, T. (2006). IL-27 induces Th1 differentiation via p38 MAPK/T-bet- and intercellular adhesion molecule-1/LFA-1/ERK1/2-dependent pathways. J Immunol 177, 7579-7587.

Owaki, T., Asakawa, M., Morishima, N., Hata, K., Fukai, F., Matsui, M., Mizuguchi, J., and Yoshimoto, T. (2005). A role for IL-27 in early regulation of Th1 differentiation. J Immunol 175, 2191-2200.

Paloneva, J., Mandelin, J., Kiialainen, A., Bohling, T., Prudlo, J., Hakola, P., Haltia, M., Konttinen, Y. T., and Peltonen, L. (2003). DAP12/TREM2 deficiency results in impaired osteoclast differentiation and osteoporotic features. J Exp Med 198, 669-675.

Paloneva, J., Manninen, T., Christman, G., Hovanes, K., Mandelin, J., Adolfsson, R., Bianchin, M., Bird, T., Miranda, R., Salmaggi, A., et al. (2002). Mutations in two genes encoding different subunits of a receptor signaling complex result in an identical disease phenotype. Am J Hum Genet 71, 656-662.

Park-Min, K. H., Ji, J. D., Antoniv, T., Reid, A. C., Silver, R. B., Humphrey, M. B., Nakamura, M., and Ivashkiv, L. B. (2009). IL-10 suppresses calcium-mediated costimulation of receptor activator NF-kappa B signaling during human osteoclast differentiation by inhibiting TREM-2 expression. J Immunol 183, 24442455.

Pascual, V., Farkas, L., and Banchereau, J. (2006). Systemic lupus erythematosus: all roads lead to type I interferons. Curr Opin Immunol 18, 676-682.

Pettit, A. R., Walsh, N. C., Manning, C., Goldring, S. R., and Gravallese, E. M. (2006). RANKL protein is expressed at the pannus-bone interface at sites of articular bone erosion in rheumatoid arthritis. Rheumatology (Oxford) 45, 1068-1076.

Pflanz, S., Hibbert, L., Mattson, J., Rosales, R., Vaisberg, E., Bazan, J. F., Phillips, J. H., McClanahan, T. K., de Waal Malefyt, R., and Kastelein, R. A. (2004). WSX-1 and glycoprotein 130 constitute a signaltransducing receptor for IL-27. J Immunol 172, 2225-2231.

Pflanz, S., Timans, J. C., Cheung, J., Rosales, R., Kanzler, H., Gilbert, J., Hibbert, L., Churakova, T., Travis, M., Vaisberg, E., et al. (2002). IL-27, a heterodimeric cytokine composed of EBI3 and p28 protein, induces proliferation of naive CD4(+) T cells. Immunity 16, 779-790.

Plenge, R. M., Seielstad, M., Padyukov, L., Lee, A. T., Remmers, E. F., Ding, B., Liew, A., Khalili, H., Chandrasekaran, A., Davies, L. R., et al. (2007). TRAF1-C5 as a risk locus for rheumatoid arthritis--a genomewide study. N Engl J Med 357, 1199-1209. 
Pratt, A. G., Isaacs, J. D., and Mattey, D. L. (2009). Current concepts in the pathogenesis of early rheumatoid arthritis. Best Pract Res Clin Rheumatol 23, 37-48.

Rawlins, B. A., Girardi, F. P., and Boachie-Adjei, O. (1998). Rheumatoid arthritis of the cervical spine. Rheum Dis Clin North Am 24, 55-65.

Reinhardt, R. L., Kang, S. J., Liang, H. E., and Locksley, R. M. (2006). T helper cell effector fates--who, how and where? Curr Opin Immunol 18, 271-277.

Remmers, E. F., Plenge, R. M., Lee, A. T., Graham, R. R., Hom, G., Behrens, T. W., de Bakker, P. I., Le, J. M., Lee, H. S., Batliwalla, F., et al. (2007). STAT4 and the risk of rheumatoid arthritis and systemic lupus erythematosus. N Engl J Med 357, 977-986.

Revaz, S., Dudler, J., and Kai-Lik So, A. (2006). Fever and musculoskeletal symptoms in an adult: differential diagnosis and management. Best Pract Res Clin Rheumatol 20, 641-651.

Romas, E., Gillespie, M. T., and Martin, T. J. (2002). Involvement of receptor activator of NFkappaB ligand and tumor necrosis factor-alpha in bone destruction in rheumatoid arthritis. Bone 30, 340-346.

Sakaguchi, S., Ono, M., Setoguchi, R., Yagi, H., Hori, S., Fehervari, Z., Shimizu, J., Takahashi, T., and Nomura, T. (2006). Foxp3+ CD25+ CD4+ natural regulatory T cells in dominant self-tolerance and autoimmune disease. Immunol Rev 212, 8-27.

Sato, K. (2008). Th17 cells and rheumatoid arthritis--from the standpoint of osteoclast differentiation. Allergol Int 57, 109-114.

Sato, K., Suematsu, A., Okamoto, K., Yamaguchi, A., Morishita, Y., Kadono, Y., Tanaka, S., Kodama, T., Akira, S., Iwakura, Y., et al. (2006). Th17 functions as an osteoclastogenic helper T cell subset that links T cell activation and bone destruction. J Exp Med 203, 2673-2682.

Scheller, J., and Rose-John, S. (2006). Interleukin-6 and its receptor: from bench to bedside. Med Microbiol Immunol 195, 173-183.

Scheller, J., Schuster, B., Holscher, C., Yoshimoto, T., and Rose-John, S. (2005). No inhibition of IL-27 signaling by soluble gp130. Biochem Biophys Res Commun 326, 724-728.

Schett, G. (2007). Erosive arthritis. Arthritis Res Ther 9 Suppl 1, S2.

Schett, G. (2009). Osteoimmunology in rheumatic diseases. Arthritis Res Ther 11, 210.

Schett, G., and Teitelbaum, S. L. (2009). Osteoclasts and arthritis. J Bone Miner Res 24, 1142-1146.

Schett, G., Zwerina, J., and Firestein, G. (2008). The p38 mitogen-activated protein kinase (MAPK) pathway in rheumatoid arthritis. Ann Rheum Dis 67, 909-916. 
Schmitz, J., Dahmen, H., Grimm, C., Gendo, C., Muller-Newen, G., Heinrich, P. C., and Schaper, F. (2000). The cytoplasmic tyrosine motifs in full-length glycoprotein 130 have different roles in IL-6 signal transduction. J Immunol 164, 848-854.

Schroder, K., Hertzog, P. J., Ravasi, T., and Hume, D. A. (2004). Interferon-gamma: an overview of signals, mechanisms and functions. J Leukoc Biol 75, 163-189.

Scott, D. L., and Kingsley, G. H. (2006). Tumor necrosis factor inhibitors for rheumatoid arthritis. N Engl J Med 355, 704-712.

Scott, D. L., Symmons, D. P., Coulton, B. L., and Popert, A. J. (1987). Long-term outcome of treating rheumatoid arthritis: results after 20 years. Lancet 1, 1108-1111.

Sengupta, T. K., Schmitt, E. M., and Ivashkiv, L. B. (1996). Inhibition of cytokines and JAK-STAT activation by distinct signaling pathways. Proc Natl Acad Sci U S A 93, 9499-9504.

Shahrara, S., Huang, Q., Mandelin, A. M., 2nd, and Pope, R. M. (2008). TH-17 cells in rheumatoid arthritis. Arthritis Res Ther 10, R93.

Sharif, M. N., Tassiulas, I., Hu, Y., Mecklenbrauker, I., Tarakhovsky, A., and Ivashkiv, L. B. (2004). IFNalpha priming results in a gain of proinflammatory function by IL-10: implications for systemic lupus erythematosus pathogenesis. J Immunol 172, 6476-6481.

Sharpe, A. H. (2009). Mechanisms of costimulation. Immunol Rev 229, 5-11.

Shinohara, M., and Takayanagi, H. (2007). Novel osteoclast signaling mechanisms. Curr Osteoporos Rep 5, 67-72.

Singh, J. A., Christensen, R., Wells, G. A., Suarez-Almazor, M. E., Buchbinder, R., Lopez-Olivo, M. A., Tanjong Ghogomu, E., and Tugwell, P. (2009). Biologics for rheumatoid arthritis: an overview of Cochrane reviews. Cochrane Database Syst Rev 4, CD007848.

Smith-Garvin, J. E., Koretzky, G. A., and Jordan, M. S. (2009). T cell activation. Annu Rev Immunol 27, 591-619.

Sokka, T. (2009). Long-term outcomes of rheumatoid arthritis. Curr Opin Rheumatol 21, 284-290.

Sorensen, M. G., Henriksen, K., Schaller, S., Henriksen, D. B., Nielsen, F. C., Dziegiel, M. H., and Karsdal, M. A. (2007). Characterization of osteoclasts derived from CD14+ monocytes isolated from peripheral blood. J Bone Miner Metab 25, 36-45.

St Clair, E. W. (1999). Interleukin 10 treatment for rheumatoid arthritis. Ann Rheum Dis 58 Suppl 1, I99I102.

St Clair, E. W. (2000). Interleukin-10: therapeutic prospects in rheumatoid arthritis. Curr Dir Autoimmun 2, 126-149. 
Steinman, L. (2007). A brief history of $T(H) 17$, the first major revision in the $T(H) 1 / T(H) 2$ hypothesis of $T$ cell-mediated tissue damage. Nat Med 13, 139-145.

Stern, P. H. (2006). The calcineurin-NFAT pathway and bone: intriguing new findings. Mol Interv 6, 193196.

Stumhofer, J. S., and Hunter, C. A. (2008). Advances in understanding the anti-inflammatory properties of IL-27. Immunol Lett 117, 123-130.

Stumhofer, J. S., Laurence, A., Wilson, E. H., Huang, E., Tato, C. M., Johnson, L. M., Villarino, A. V., Huang, Q., Yoshimura, A., Sehy, D., et al. (2006). Interleukin 27 negatively regulates the development of interleukin 17-producing $\mathrm{T}$ helper cells during chronic inflammation of the central nervous system. Nat Immunol 7, 937-945.

Stumhofer, J. S., Silver, J. S., Laurence, A., Porrett, P. M., Harris, T. H., Turka, L. A., Ernst, M., Saris, C. J., O'Shea, J. J., and Hunter, C. A. (2007). Interleukins 27 and 6 induce STAT3-mediated T cell production of interleukin 10. Nat Immunol 8, 1363-1371.

Takayanagi, H. (2007a). Osteoimmunology: shared mechanisms and crosstalk between the immune and bone systems. Nat Rev Immunol 7, 292-304.

Takayanagi, H. (2007b). The role of NFAT in osteoclast formation. Ann N Y Acad Sci 1116, 227-237.

Takayanagi, H., Kim, S., Koga, T., Nishina, H., Isshiki, M., Yoshida, H., Saiura, A., Isobe, M., Yokochi, T., Inoue, J., et al. (2002a). Induction and activation of the transcription factor NFATc1 (NFAT2) integrate RANKL signaling in terminal differentiation of osteoclasts. Dev Cell 3, 889-901.

Takayanagi, H., Kim, S., Matsuo, K., Suzuki, H., Suzuki, T., Sato, K., Yokochi, T., Oda, H., Nakamura, K., Ida, N., et al. (2002b). RANKL maintains bone homeostasis through c-Fos-dependent induction of interferon-beta. Nature 416, 744-749.

Takayanagi, H., Kim, S., and Taniguchi, T. (2002c). Signaling crosstalk between RANKL and interferons in osteoclast differentiation. Arthritis Res 4 Suppl 3, S227-232.

Takayanagi, H., Ogasawara, K., Hida, S., Chiba, T., Murata, S., Sato, K., Takaoka, A., Yokochi, T., Oda, H., Tanaka, K., et al. (2000). T-cell-mediated regulation of osteoclastogenesis by signalling cross-talk between RANKL and IFN-gamma. Nature 408, 600-605.

Takayanagi, H., Sato, K., Takaoka, A., and Taniguchi, T. (2005). Interplay between interferon and other cytokine systems in bone metabolism. Immunol Rev 208, 181-193.

Takeda, A., Hamano, S., Yamanaka, A., Hanada, T., Ishibashi, T., Mak, T. W., Yoshimura, A., and Yoshida, H. (2003). Cutting edge: role of IL-27/WSX-1 signaling for induction of T-bet through activation of STAT1 during initial Th1 commitment. J Immunol 170, 4886-4890. 
Taylor, P. C., and Feldmann, M. (2009). Anti-TNF biologic agents: still the therapy of choice for rheumatoid arthritis. Nat Rev Rheumatol 5, 578-582.

Teitelbaum, S. L. (2000). Bone resorption by osteoclasts. Science 289, 1504-1508.

Teitelbaum, S. L. (2004). RANKing c-Jun in osteoclast development. J Clin Invest 114, 463-465.

Theofilopoulos, A. N. (1995a). The basis of autoimmunity: Part I. Mechanisms of aberrant self-recognition. Immunol Today 16, 90-98.

Theofilopoulos, A. N. (1995b). The basis of autoimmunity: Part II. Genetic predisposition. Immunol Today $16,150-159$.

Tracey, D., Klareskog, L., Sasso, E. H., Salfeld, J. G., and Tak, P. P. (2008). Tumor necrosis factor antagonist mechanisms of action: a comprehensive review. Pharmacol Ther 117, 244-279.

Turesson, C., and Matteson, E. L. (2009). Vasculitis in rheumatoid arthritis. Curr Opin Rheumatol 21, 3540.

Vallabhapurapu, S., and Karin, M. (2009). Regulation and function of NF-kappaB transcription factors in the immune system. Annu Rev Immunol 27, 693-733.

Vallbracht, I., and Helmke, K. (2005). Additional diagnostic and clinical value of anti-cyclic citrullinated peptide antibodies compared with rheumatoid factor isotypes in rheumatoid arthritis. Autoimmun Rev 4, 389-394.

van der Pouw Kraan, T. C., van Gaalen, F. A., Kasperkovitz, P. V., Verbeet, N. L., Smeets, T. J., Kraan, M. C., Fero, M., Tak, P. P., Huizinga, T. W., Pieterman, E., et al. (2003). Rheumatoid arthritis is a heterogeneous disease: evidence for differences in the activation of the STAT-1 pathway between rheumatoid tissues. Arthritis Rheum 48, 2132-2145.

van Holten, J., Pavelka, K., Vencovsky, J., Stahl, H., Rozman, B., Genovese, M., Kivitz, A. J., Alvaro, J., Nuki, G., Furst, D. E., et al. (2005). A multicentre, randomised, double blind, placebo controlled phase II study of subcutaneous interferon beta-1a in the treatment of patients with active rheumatoid arthritis. Ann Rheum Dis 64, 64-69.

van Oosterhout, M., Bajema, I., Levarht, E. W., Toes, R. E., Huizinga, T. W., and van Laar, J. M. (2008). Differences in synovial tissue infiltrates between anti-cyclic citrullinated peptide-positive rheumatoid arthritis and anti-cyclic citrullinated peptide-negative rheumatoid arthritis. Arthritis Rheum 58, 53-60.

van Venrooij, W. J., Vossenaar, E. R., and Zendman, A. J. (2004). Anti-CCP antibodies: the new rheumatoid factor in the serology of rheumatoid arthritis. Autoimmun Rev 3 Suppl 1, S17-19.

van Vollenhoven, R. F. (2009). Treatment of rheumatoid arthritis: state of the art 2009. Nat Rev Rheumatol $5,531-541$. 
Vang, T., Miletic, A. V., Arimura, Y., Tautz, L., Rickert, R. C., and Mustelin, T. (2008). Protein tyrosine phosphatases in autoimmunity. Annu Rev Immunol 26, 29-55.

Villarino, A., Hibbert, L., Lieberman, L., Wilson, E., Mak, T., Yoshida, H., Kastelein, R. A., Saris, C., and Hunter, C. A. (2003). The IL-27R (WSX-1) is required to suppress T cell hyperactivity during infection. Immunity 19, 645-655.

Villarino, A. V., Artis, D., Bezbradica, J. S., Miller, O., Saris, C. J., Joyce, S., and Hunter, C. A. (2008). IL27R deficiency delays the onset of colitis and protects from helminth-induced pathology in a model of chronic IBD. Int Immunol 20, 739-752.

Villarino, A. V., Huang, E., and Hunter, C. A. (2004). Understanding the pro- and anti-inflammatory properties of IL-27. J Immunol 173, 715-720.

Villarino, A. V., and Hunter, C. A. (2004). Biology of recently discovered cytokines: discerning the proand anti-inflammatory properties of interleukin-27. Arthritis Res Ther 6, 225-233.

Wada, T., Nakashima, T., Hiroshi, N., and Penninger, J. M. (2006). RANKL-RANK signaling in osteoclastogenesis and bone disease. Trends Mol Med 12, 17-25.

Wagner, E. F., and Eferl, R. (2005). Fos/AP-1 proteins in bone and the immune system. Immunol Rev 208, 126-140.

Wang, R., Han, G., Wang, J., Chen, G., Xu, R., Wang, L., Li, X., Shen, B., and Li, Y. (2008). The pathogenic role of interleukin-27 in autoimmune diabetes. Cell Mol Life Sci 65, 3851-3860.

Weaver, C. T., Harrington, L. E., Mangan, P. R., Gavrieli, M., and Murphy, K. M. (2006). Th17: an effector CD4 T cell lineage with regulatory T cell ties. Immunity 24, 677-688.

Weinblatt, M. E., Keystone, E. C., Furst, D. E., Moreland, L. W., Weisman, M. H., Birbara, C. A., Teoh, L. A., Fischkoff, S. A., and Chartash, E. K. (2003). Adalimumab, a fully human anti-tumor necrosis factor alpha monoclonal antibody, for the treatment of rheumatoid arthritis in patients taking concomitant methotrexate: the ARMADA trial. Arthritis Rheum 48, 35-45.

Williams, R. O., Feldmann, M., and Maini, R. N. (1992). Anti-tumor necrosis factor ameliorates joint disease in murine collagen-induced arthritis. Proc Natl Acad Sci U S A 89, 9784-9788.

Wirtz, S., Becker, C., Fantini, M. C., Nieuwenhuis, E. E., Tubbe, I., Galle, P. R., Schild, H. J., Birkenbach, M., Blumberg, R. S., and Neurath, M. F. (2005). EBV-induced gene 3 transcription is induced by TLR signaling in primary dendritic cells via NF-kappa B activation. J Immunol 174, 2814-2824.

Wong, B. R., Besser, D., Kim, N., Arron, J. R., Vologodskaia, M., Hanafusa, H., and Choi, Y. (1999). TRANCE, a TNF family member, activates Akt/PKB through a signaling complex involving TRAF6 and c-Src. Mol Cell 4, 1041-1049. 
Wong, B. R., Josien, R., Lee, S. Y., Vologodskaia, M., Steinman, R. M., and Choi, Y. (1998). The TRAF family of signal transducers mediates NF-kappaB activation by the TRANCE receptor. J Biol Chem 273, 28355-28359.

Wong, B. R., Rho, J., Arron, J., Robinson, E., Orlinick, J., Chao, M., Kalachikov, S., Cayani, E., Bartlett, F. S., 3rd, Frankel, W. N., et al. (1997). TRANCE is a novel ligand of the tumor necrosis factor receptor family that activates c-Jun N-terminal kinase in T cells. J Biol Chem 272, 25190-25194.

Wu, Y., Tworkoski, K., Michaud, M., and Madri, J. A. (2009). Bone marrow monocyte PECAM-1 deficiency elicits increased osteoclastogenesis resulting in trabecular bone loss. J Immunol 182, 2672-2679.

Xing, L., Schwarz, E. M., and Boyce, B. F. (2005). Osteoclast precursors, RANKL/RANK, and immunology. Immunol Rev 208, 19-29.

Yago, T., Nanke, Y., Ichikawa, N., Kobashigawa, T., Mogi, M., Kamatani, N., and Kotake, S. (2009). IL17 induces osteoclastogenesis from human monocytes alone in the absence of osteoblasts, which is potently inhibited by anti-TNF-alpha antibody: A novel mechanism of osteoclastogenesis by IL-17. J Cell Biochem.

Yasuda, H., Shima, N., Nakagawa, N., Yamaguchi, K., Kinosaki, M., Mochizuki, S., Tomoyasu, A., Yano, K., Goto, M., Murakami, A., et al. (1998). Osteoclast differentiation factor is a ligand for osteoprotegerin/osteoclastogenesis-inhibitory factor and is identical to TRANCE/RANKL. Proc Natl Acad Sci U S A 95, 3597-3602.

Yazici, Y. (2009). Treatment of rheumatoid arthritis: we are getting there. Lancet 374, 178-180.

Yazici, Y., and Yazici, H. (2008). Tumor necrosis factor alpha inhibitors, methotrexate or both? An inquiry into the formal evidence for when they are to be used in rheumatoid arthritis. Clin Exp Rheumatol 26, 449452.

Yeo, H., McDonald, J. M., and Zayzafoon, M. (2006). NFATc1: a novel anabolic therapeutic target for osteoporosis. Ann N Y Acad Sci 1068, 564-567.

Yoshida, H., and Miyazaki, Y. (2008). Regulation of immune responses by interleukin-27. Immunol Rev 226, 234-247.

Yoshida, H., Miyazaki, Y., Wang, S., and Hamano, S. (2007). Regulation of Defense Responses against Protozoan Infection by Interleukin-27 and Related Cytokines. J Biomed Biotechnol 2007, 79401.

Yoshida, H., Nakaya, M., and Miyazaki, Y. (2009). Interleukin 27: a double-edged sword for offense and defense. J Leukoc Biol.

Yoshimura, A., Naka, T., and Kubo, M. (2007). SOCS proteins, cytokine signalling and immune regulation. Nat Rev Immunol 7, 454-465. 
Zenz, R., Eferl, R., Scheinecker, C., Redlich, K., Smolen, J., Schonthaler, H. B., Kenner, L., Tschachler, E., and Wagner, E. F. (2008). Activator protein 1 (Fos/Jun) functions in inflammatory bone and skin disease. Arthritis Res Ther 10, 201.

Zhao, Q., Shao, J., Chen, W., and Li, Y. P. (2007). Osteoclast differentiation and gene regulation. Front Biosci 12, 2519-2529.

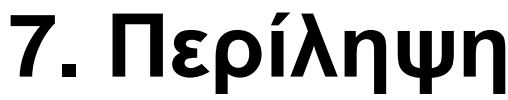




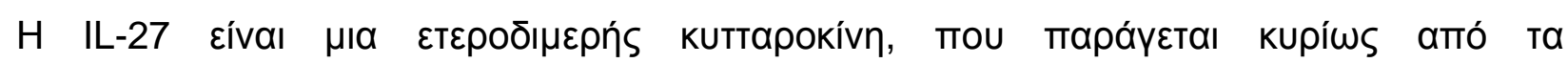

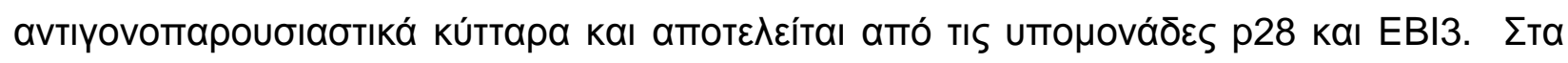

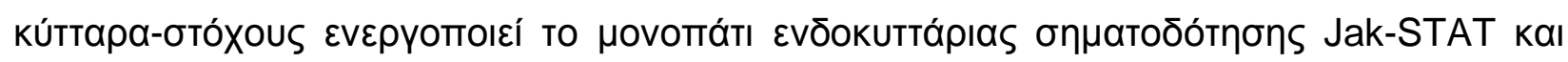

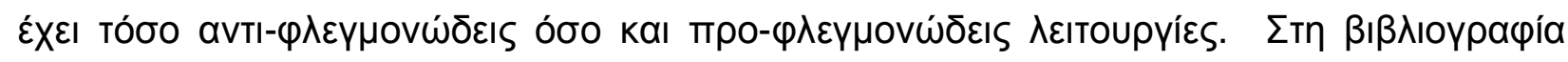

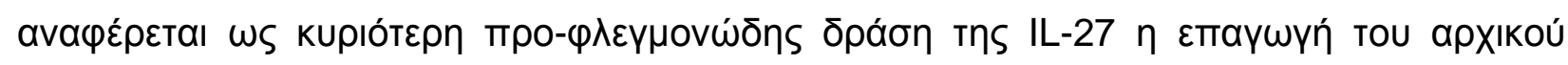

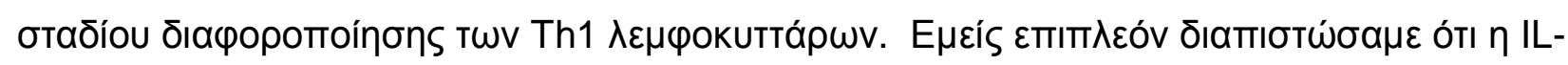

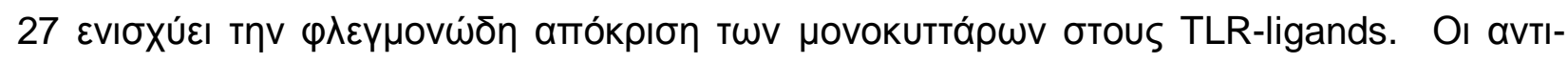

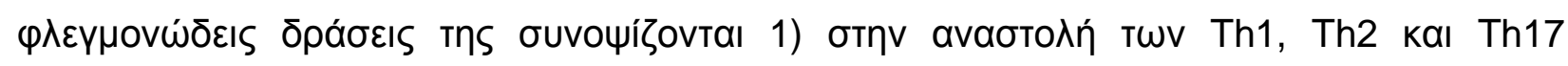

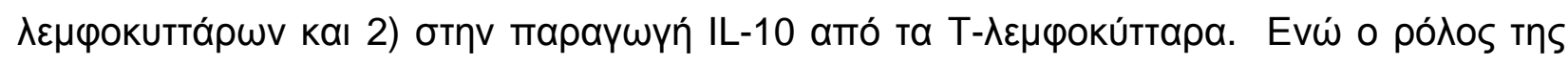

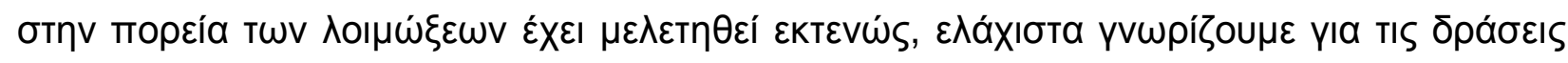

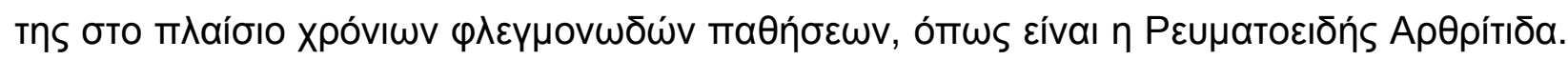

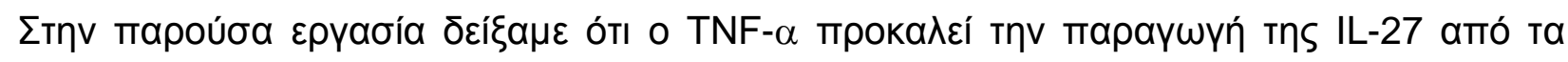

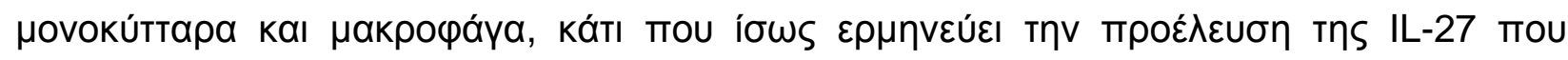

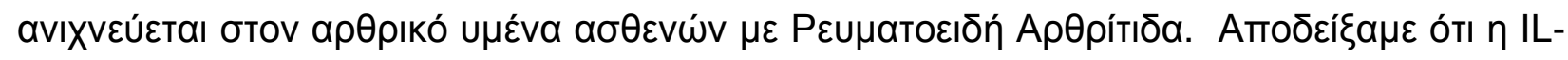

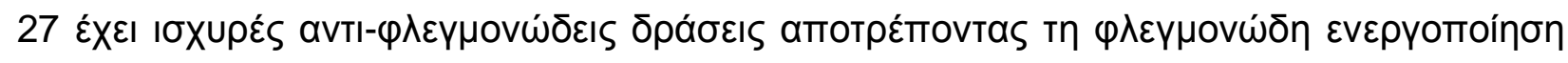

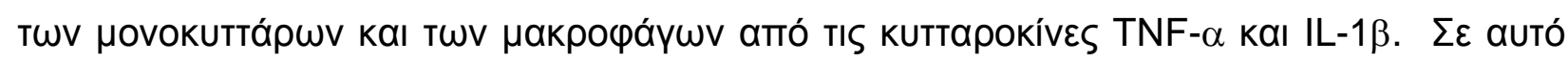

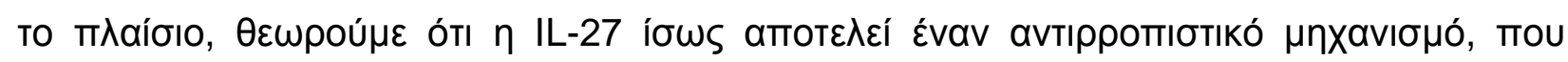

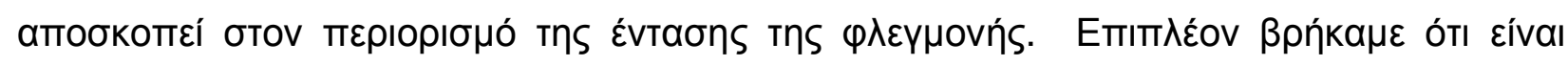

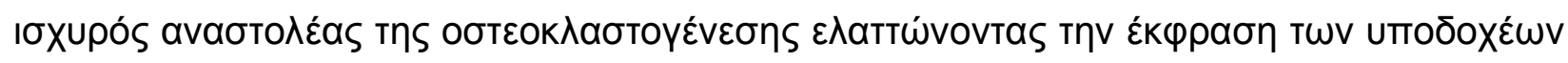

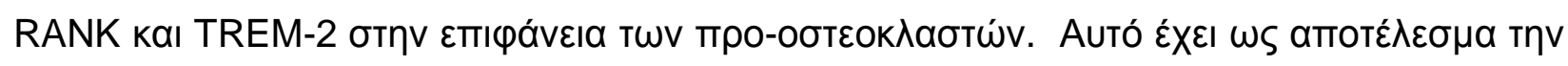




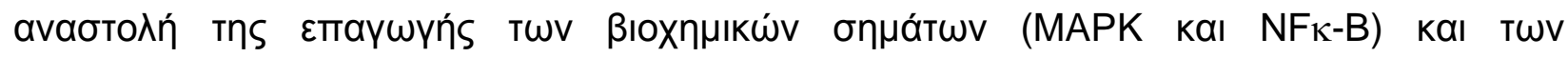

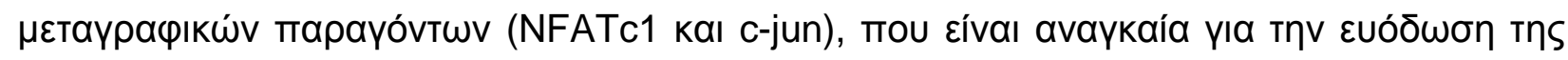

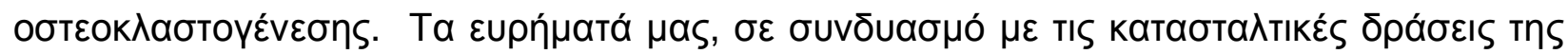

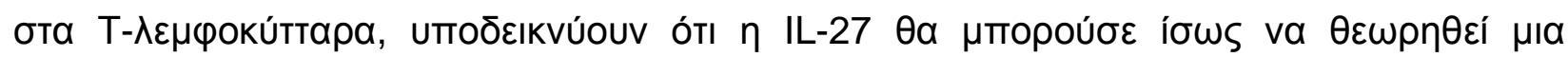

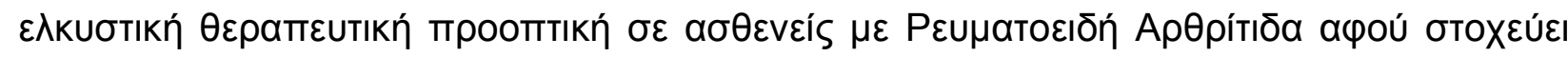

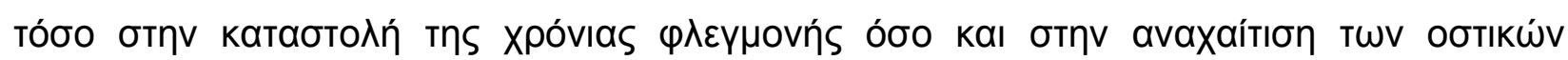

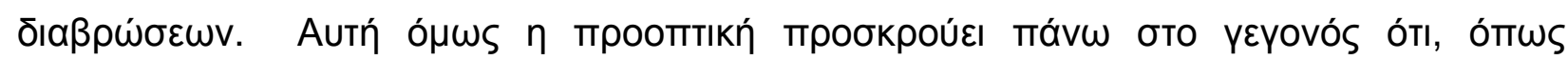

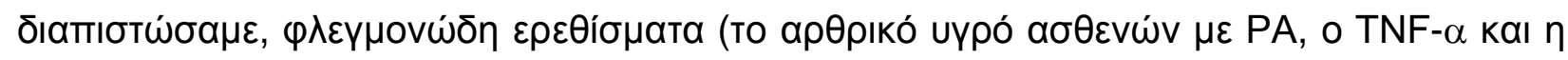

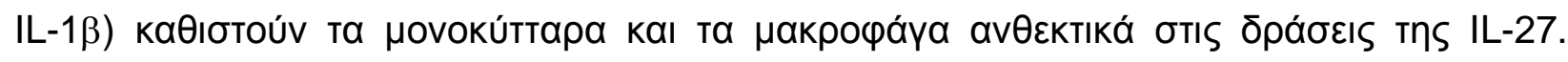

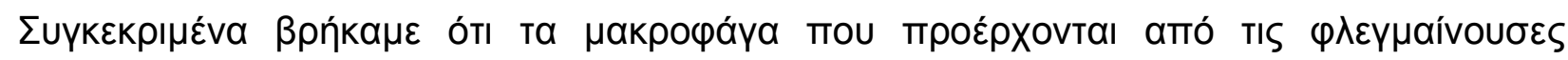

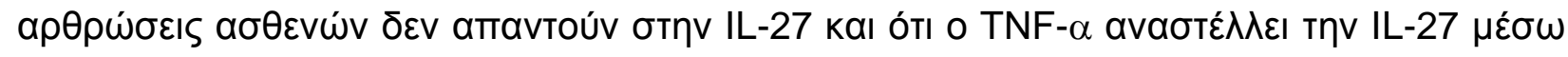

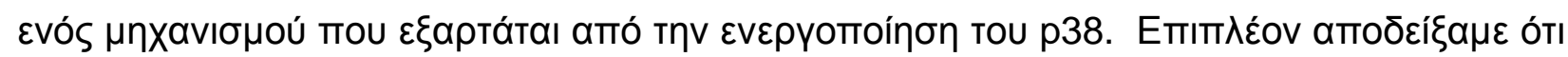

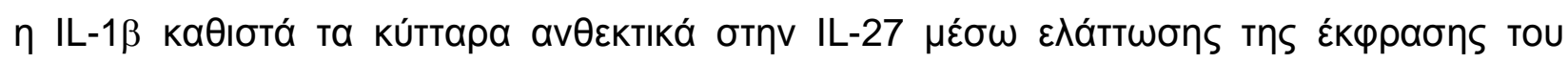

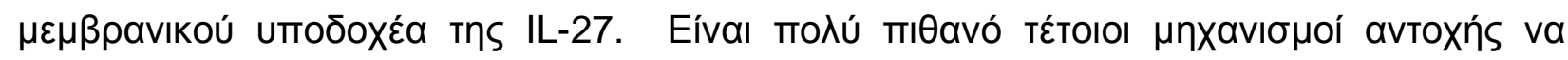

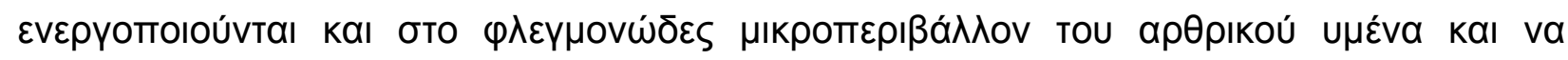

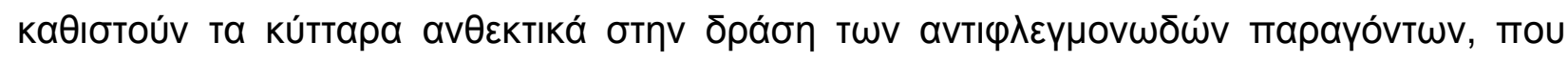

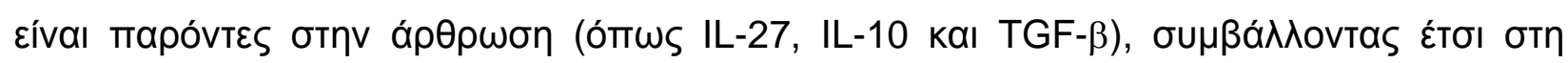

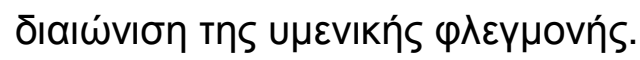


8. Summary 
IL-27 is a cytokine mainly produced by antigen presenting cells and is comprised of p28 and EBI3 subunits. IL-27 activates the Jak-STAT signaling pathway and has both antiand pro-inflammatory functions. The main pro-inflammatory function of IL-27 is the initiation of Th1 differntiation. We have also found that IL-27 primes human monocytes for more robust responses to TLR-ligands. The dominant anti-inflammatory functions of IL-27 are: 1) the inhibition of Th1, Th2 and Th17 differentiation and 2) the production of IL-10 by lymphocytes. While the functions of IL-27 in the course of infections are well-known, the potential role of IL-27 in chronic inflammatory diseases, like Rheumatoid Arthritis (RA), is still unclear. In our current study we have found that TNF- $\alpha$ stimulates production of IL-27 from monocytes and macrophages and this is a potential explanation for the reported expression of IL-27 in synovial tissues from patients with RA. We have also found that IL27 has potent anti-inflammatory functions by inhibiting the effects of TNF- $\alpha$ and IL-1 $1 \beta$ on human monocytes and macrophages. In this context we hypothesize that IL-27 represents a feed-back inhibition mechanism that aims to restrain inflammation. Additionally, we observed that IL-27 is a potent inhibitor of human osteoclastogenesis by down-regulating the expression of RANK and TREM-2 on osteoclast precursors and abrogating RANKLmediated activation of MAPK and NFK-B pathways and induction of NFATc1 and c-jun. Our observations suggest that IL-27, by targeting concurrently inflammation and joint destruction, could be a potential attractive therapeutic strategy for RA. The main limitation for this perspective is that inflammatory stimuli (including synovial fluids derived from RA patients, TNF- $\alpha$ and IL-1 $\beta$ ) render monocytes and macrophages refractory to the 
regulatory functions of IL-27. We have found that: 1) synovial macrophages derived from RA do not respond to IL-27 and 2) TNF- $\alpha$ and IL-1 $\beta$ inhibit IL-27 functions by a p38dependent mechanism and by down-regulating expression of IL-27 receptor respectively. In rheumatoid arthritis, within the inflammed synovium, p38 is active and the cytokines TNF- $\alpha$ and IL-1 $\beta$ are abundant. In this context, we believe that these inflammatory stimuli render cells refractory to the anti-inflammatory factors that are expressed within the synovial microenvironment (including IL-27, IL-10 and TGF- $\beta$ ), contributing to the perpetuation of joint inflammation. 
9. А 


\title{
IL-27 Activates Human Monocytes via STAT1 and Suppresses IL-10 Production but the Inflammatory Functions of IL-27 Are Abrogated by TLRs and p38
}

\author{
George D. Kalliolias* and Lionel B. Ivashkiv ${ }^{2}{ }^{\dagger}$
}

IL-27 is a member of the IL-12 family of cytokines that activates the Jak-STAT signaling pathway in a context-dependent manner and has pleiotropic effects on acquired immunity. IL-27 has the capacity to promote early stages of Th1 generation, but recent evidence has suggested a predominant suppressive effect on Th1, Th2, and Th17 differentiation. Although modest suppressive effects of IL-27 on myeloid lineage cells have been observed, there is limited knowledge about the role of IL-27 in the regulation of innate immunity. In this study we report that although in resting murine macrophages IL-27 had minimal if any effects, in resting human monocytes IL-27 had profound proinflammatory functions. IL-27 activated a STAT1-dominant pattern of signaling in human monocytes with the consequent activation of STAT1-dependent inflammatory target genes. IL-27 primed monocytes for augmented responses to TLR stimulation in a STAT1-dependent manner, altered IL-10 signaling, and attenuated IL-10-induced gene expression. Strikingly, IL-27 strongly suppressed TLR-induced IL-10 production in human monocytes. However, the proinflammatory effects of IL-27 on human monocytes were rapidly abrogated by LPS via a p38-mediated mechanism that inhibited IL-27 signaling. Our findings identify a predominantly proinflammatory function for IL-27 in human monocytes and suggest a mechanism by which the activating effects of IL-27 on innate immunity are attenuated as an immune response proceeds and IL-27 transitions to predominantly suppressive effects on acquired immunity. The Journal of Immunology, 2008, 180: 6325-6333.

I nterleukin-27 is a member of the IL-12 family of cytokines that are produced by APCs and play a key role in regulating T cell differentiation and function (1). IL-27 is predominantly produced early after stimulation of APCs by TLR ligands or infectious agents. Initial studies of IL-27 function revealed a role in promoting early stages of activation of naive $\mathrm{CD} 4{ }^{+}$cells and differentiation to the Th1 lineage (2-4). It was proposed that IL-27 amplifies early responses to weak infectious stimuli to achieve a robust response (5). More recent work has highlighted a regulatory role for IL-27 in suppressing acquired immunity. Indeed, IL-27 suppresses the development of Th1, Th2, and Th17 T cell subsets, and mice deficient in the IL-27 receptor exhibit enhanced inflammation and related toxicity in several infectious disease models (6-8). Strikingly, IL-27 has recently been shown to induce IL-10 production in activated T cells (9-11), thus identifying one mechanism for the suppressive effects of IL-27. However, the molecular basis for its pleiotropic actions during the course of immune responses has not been elucidated.

*Arthritis and Tissue Degeneration Program, Department of Medicine, Hospital for Special Surgery, New York, NY 10021; and Graduate Program in Immunology, Weill Graduate School of Medical Sciences of Cornell University, New York, NY 10021

Received for publication December 18, 2007. Accepted for publication February 28, 2008.

The costs of publication of this article were defrayed in part by the payment of page charges. This article must therefore be hereby marked advertisement in accordance with 18 U.S.C. Section 1734 solely to indicate this fact.

${ }^{1}$ This work was supported by The Stavros S. Niarchos International Fellowship Exchange Program (to G.D.K.) and by a grant from the National Institutes of Health (to L.B.I.).

${ }^{2}$ Address correspondence and reprint requests to Dr. Lionel B. Ivashkiv, Department of Medicine, Hospital for Special Surgery, 535 East 70th Street, New York, NY 10021. E-mail address: ivashkivl@hss.edu

Copyright $\odot 2008$ by The American Association of Immunologists, Inc. 0022-1767/08/\$2.00
IL-27 is a heterodimeric cytokine comprised of EBI3 (EBVinduced protein 3) and p28 subunits that share similarity with the p40 and p35 subunits of IL-12, respectively (12). The IL-27 receptor is a heterodimer composed of a WSX-1 subunit (also termed TCCR for T cell cytokine receptor), which confers ligand specificity, and the gp130 signaling subunit, which is also used by the IL- 6 family of cytokines and activates the Jak-STAT signal transduction pathway (13). IL-27 activates STATs in a contextdependent manner, depending on cell type and activation state. In resting lymphocytes IL-27 activates STAT1, STAT3, STAT5, and low amounts of STAT4 $(14,15)$, whereas activation of STAT1 is decreased in fully activated relative to resting $\mathrm{CD} 4^{+} \mathrm{T}$ cells (16). STAT1 plays a key role in mediating the suppressive effects of IL-27 on Th17 (17-19) and Th2 differentiation (15), whereas both STAT1 and STAT3 are required for IL-27-induced IL-10 production in T cells $(10,11)$. In myeloid cells, IL-27-induced phosphorylation of STAT1 and STAT3 has been observed (8, 13, 20-22), but activation of these STATs has not been linked to IL-27 function in the myeloid lineage.

STAT1 and STAT3 have many antagonistic functions in myeloid cells. For example, STAT1 mediates inflammatory, proapoptotic and antiproliferative effects, while STAT3 mediates antiinflammatory, antiapoptotic, and proliferative effects $(22,23)$. Several cytokines, including IFN- $\gamma$, IFN- $\alpha \beta$, IL-6 and IL-10, activate both STAT1 and STAT3 in myeloid cells, and we and others have proposed that the relative balance of STAT1 and STAT3 activation by a given cytokine will determine its proinflammatory vs anti-inflammatory functional profile (24-30). For example, proinflammatory IFN- $\gamma$ activates predominantly STAT1 (31) while the potent anti-inflammatory IL-10 activates predominantly STAT3 $(22,32)$. More pleiotropic cytokines, such as IL-6 and IFN- $\alpha$, activate both STAT1 and STAT3 $(33,34)$ and we have shown that conditions that augment STAT1 activation, such as 
priming macrophages $(\mathrm{M} \phi \mathrm{s})^{3}$ with IFN- $\gamma$, increase the proinflammatory properties of cytokines and can even confer activating functions to IL-10 $(24,26,35)$. IL-27 activates both STAT1 and STAT3 in myeloid cells and thus has the potential to induce both proinflammatory and anti-inflammatory effects.

There is limited knowledge about the role of IL-27 in the regulation of innate immunity. An early study showed that IL-27 can induce proinflammatory cytokines in human monocytes and mast cells (13). Follow-up studies in murine systems have suggested a moderately suppressive function of IL-27 on activated myeloid cells. IL-27 inhibited the function of murine mast cells (36), partially suppressed the production of proinflammatory cytokines by activated murine M $\phi \mathrm{s}$ (8), and attenuated the Ag-presenting capacity and Th1-promoting function of activated murine dendritic cells in vitro (21). We investigated the function and signaling of IL-27 in monocytes and $\mathrm{M} \phi \mathrm{s}$. We found that resting murine $\mathrm{M} \phi$ are minimally responsive to IL-27, but human monocytes are strongly activated by IL-27 in a STAT1-dominant manner. IL-27 primed human monocytes to produce augmented inflammatory responses to TLR ligation, strongly suppressed TLR-induced IL-10 production, and altered IL-10 function by increasing IL-10-induced STAT1 activation and attenuating IL-10-induced gene expression. IL-27 signaling to STAT1 and downstream gene activation were rapidly abrogated by TLR signaling via a p38-dependent mechanism. These results identify STAT1-dependent inflammatory functions for IL-27 in human monocytes and suggest a mechanism by which the inflammatory properties of IL-27 are extinguished as the immune response proceeds and IL-27 assumes a more regulatory role.

\section{Materials and Methods \\ Cell culture}

Bone marrow-derived $\mathrm{M} \phi \mathrm{s}$, resident peritoneal $\mathrm{M} \phi \mathrm{s}$, and splenocytes were obtained as described (25) from C57BL/6J mice (The Jackson Laboratory). Cells were cultured in DMEM supplemented with 20\% FBS (HyClone), $100 \mathrm{U} / \mathrm{ml}$ penicillin, and streptomycin in the presence or absence of 10 $\mathrm{ng} / \mathrm{ml}$ murine M-CSF (PeproTech). The following murine ( $\mathrm{m}$ ) cytokines were used to stimulate cells as indicated: $\mathrm{mIFN}-\gamma(100 \mathrm{U} / \mathrm{ml})$ (PeproTech), mIL-27 (100 ng/ml), and mIL-6 (50 ng/ml) (R\&D Systems). PBMCs were obtained from whole blood from healthy volunteers by density gradient centrifugation using Ficoll (Invitrogen). CD14 ${ }^{+}$monocytes were purified from fresh PBMCs using anti-CD14 magnetic beads (Miltenyi Biotec) as recommended by the manufacturer. Purity of monocytes was $>97 \%$ as verified by FACS. Cells were cultured in RPMI 1640 medium (Invitrogen) supplemented with 10\% FBS (HyClone), $100 \mathrm{U} / \mathrm{ml}$ penicillin, and streptomycin in the presence or absence of $10 \mathrm{ng} / \mathrm{ml}$ human (h) M-CSF (hMCSF) (PeproTech). The following human cytokines were used to stimulate cells as indicated: hIFN- $\gamma(100 \mathrm{U} / \mathrm{ml})$ (Roche), hIL-10 (100 ng/ ml) (PeproTech), hIL-27 (100 ng/ml) (R\&D Systems), hIL-6 (50 ng/ml) (R\&D Systems), and soluble IL-6R (100 ng/ml). Monocytes were stimulated with $(S)$-[2,3-bis(palmitoyloxy)-(2-RS)-propyl]- $N$-palmitoyl- $(R)$ Cys- $(S)$-Ser- $(S)-\mathrm{Lys}_{4}-\mathrm{OH}, 3 \mathrm{HCl}$ ( $\mathrm{Pam}_{3} \mathrm{Cys}$; ECM Microcollections), LPS (Sigma), CL097 (InvivoGen), and zymosan (Molecular Probes) as indicated in the figure legends. In some experiments cells were pretreated with cycloheximide (A.G. Scientific), actinomycin D (Sigma), the p38 inhibitor SB203580, or the MEK inhibitor U0126 (Calbiochem). The experiments using human and murine cells were approved by the Hospital for Special Surgery Institutional Review Board and Institutional Animal Care and Use Committee (New York, NY), respectively.

\footnotetext{
${ }^{3}$ Abbreviations used in this paper: $\mathrm{M} \phi$, macrophage; BMDM, bone marrow-derived macrophage; CR1, complement receptor 1; DEPP, decidual protein-induced progesterone; h (prefix), human; IRF-1, IFN regulatory factor $1 ; \mathrm{m}$ (prefix), murine;

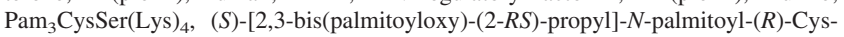
$(S)$-Ser- $(S)$-Lys $4-\mathrm{OH}, 3 \mathrm{HCl} ; \mathrm{PGDH}, \quad 15$-hydroxy prostaglandin dehydrogenase; SHP-2, Src homology domain 2-containing phosphatase; shRNA, short hairpin RNA; SOCS, suppressor of cytokine signaling.
}

\section{Immunoblotting and ELISA}

Whole cell extracts were obtained and protein levels were quantitated using the Bradford assay (Bio-Rad). For immunoblotting, $5 \mu \mathrm{g}$ of whole cell lysates were fractionated on $7.5 \%$ polyacrylamide gels using SDS-PAGE, transferred to polyvinylidene fluoride membranes (Millipore), and incubated with specific Abs. ECL was used for detection. mAbs against STAT1 and STAT3 were purchased from BD Transduction Laboratories. Phosphorylation-specific (tyrosine 701) STAT1 Ab and phosphorylation-specific (tyrosine 705) STAT3 Ab were obtained from Cell Signaling Technology. For ELISA, paired TNF- $\alpha$, IL-6, and IL-10 capture and detection Abs were purchased from R\&D Systems and used in a sandwich ELISA according to the instructions of the manufacturer.

\section{Real-time quantitative RT-PCR}

For real-time PCR, total RNA was extracted using an RNeasy mini kit (Qiagen) and $1 \mu \mathrm{g}$ of total RNA was reverse transcribed using a first strand cDNA synthesis kit (Fermentas). Quantitative PCR was performed using iQ SYBR Green Supermix and iCycler iQ thermal cycler (Bio-Rad) following the manufacturer's protocols. Triplicate reactions were run for each sample and expression of a tested gene was normalized relative to levels of GAPDH. The oligonucleotide primers used are as follows: mGAPDH, 5' ATCAAGAAGGTGGTGAAGCA-3' (forward) and 5'-AGACAACCTG GTCCTCAGTGT-3' (reverse); murine suppressor of cytokine signaling (SOCS) 1, 5' -CATCCCTCTTAACCCGGTAC-3' (forward) and 5'-TGGA AGGGGAAGGAACTCAG-3' (reverse); mSOCS3, 5' -ATTTGCCTCAAT CACTTTTAT-3' (forward) and 5'-ACTGGGATTTGGTTGAGTTT-3' (reverse); murine IFN regulatory factor 1 (IRF-1), 5' -CCCACAGAAGAG CATAGCAC-3' (forward) and 5'-AGCAGTTCTTTGGGAATAGG-3' (reverse); mouse inducible protein-10 (IP-10), 5'-ATTCTTTAAGGGCT GGTCTGA-3' (forward) and 5'-CACCTCCACATAGCTTACAGT-3' (reverse); hGAPDH, 5'-ATCAAGAAGGTGGTGAAGCA-3' (forward) and 5'-GTCGCTGTTGAAGTCAGAGGA-3' (reverse); hSOCS1, 5'-TG TTGTAGCAGCTTAACTGTATC-3' (forward) and 5'-AGAGGTAGGA GGTGCGAGT-3' (reverse); hSOCS3, 5'-CACTCTTCAGCATCTCTGTC GGAAG-3' (forward) and 5'-CATAGGAGTCCAGGTGGCCGTT GAC-3' (reverse); hCXCL10, 5'-ATTTGCTGCCTTATCTTTCTG-3' (forward) and 5'-TCTCACCCTTCTTTTTCATTGTAG-3' (reverse); human complement receptor 1 (CR-1), 5'-GGATCCCTGCTGGCGGTT GTG-3' (forward) and 5'-CCAGGGCGGCATTCATAGTTCAGA-3' (reverse); human decidual protein-induced progesterone (DEPP), 5'-GGCC GCCCTGCTGTGTCC-3' (forward) and 5'-CCTCTGGCTTGGCTGCTT TTCCTG-3' (reverse); hCD163, 5'-CCAGTCCCAAACACTGTCCT-3' (forward) and 5'-CACTCTCTATGCAGGCCACA-3' (reverse); human 15-hydroxy prostaglandin dehydrogenase (PDGH), 5'-GCTGGAGTGAA TAATGAGAAAAAC-3' (forward) and 5'-CAATAAACCGGCTGCT GTG-3' (reverse); hCXCL9, 5'-ATCAGCACCAACCAAGGGACT-3' (forward) and 5'-GCTTTTTCTTTTGGCTGACCTG-3' (reverse); hSTAT1, 5' -TGGGTTTGACAAGGTTCTT-3' (forward) and 5' -TATGCA GTGCCACGGAAAG-3' (reverse); hIRF-1, 5'-GCACTAAGCGAAAATT GCA-3' (forward) and 5'-GGGAGTTTCCTTCACATTCA-3' (reverse); hTNF- $\alpha, 5^{\prime}$-AATAGGCTGTTCCCATGTAGC-3' (forward) and 5'-AG AGGCTCAGCAATGAGTGA-3' (reverse); hIL-6, 5' -TAATGGGCATTC CTTCTTCT-3' (forward) and 5' -TGTCCTAACGCTCATACTTTT-3' (reverse); hIL-10, 5'-TTATCTTGTCTCTGGGCTTGG-3' (forward) and 5' GTTGGGGAATGAGGTTAGGG-3' (reverse).

\section{Results}

Nonactivated murine $M \phi$ s are minimally responsive to $I L-27$

IL-27 strongly suppresses murine T cells via STAT1 and STAT3 $(12,16)$, and IL-27-induced STAT3 activation, along with moderately suppressive effects on activation-induced cytokine production, has been described in murine myeloid cells (8). We wished to investigate the effects of IL-27 on resting M $\phi$ s not exposed to activation stimuli. We analyzed IL-27-induced activation of STAT tyrosine phosphorylation and downstream gene expression. Stimulation of murine splenocytes, which are comprised predominantly of lymphocytes, with IL-27 rapidly induced tyrosine phosphorylation of STAT1 and STAT3 as expected (Fig. 1A). Surprisingly, tyrosine phosphorylation of STAT1 and STAT3 was not observed after the stimulation of bone marrow-derived M $\phi$ s (BMDMs) with the same preparation of IL-27 in parallel wells in the same experiment (Fig. 1B), and similar results were obtained using resident peritoneal $\mathrm{M} \phi$ (data not shown). In contrast, IFN- $\gamma$ and IL-6, used 

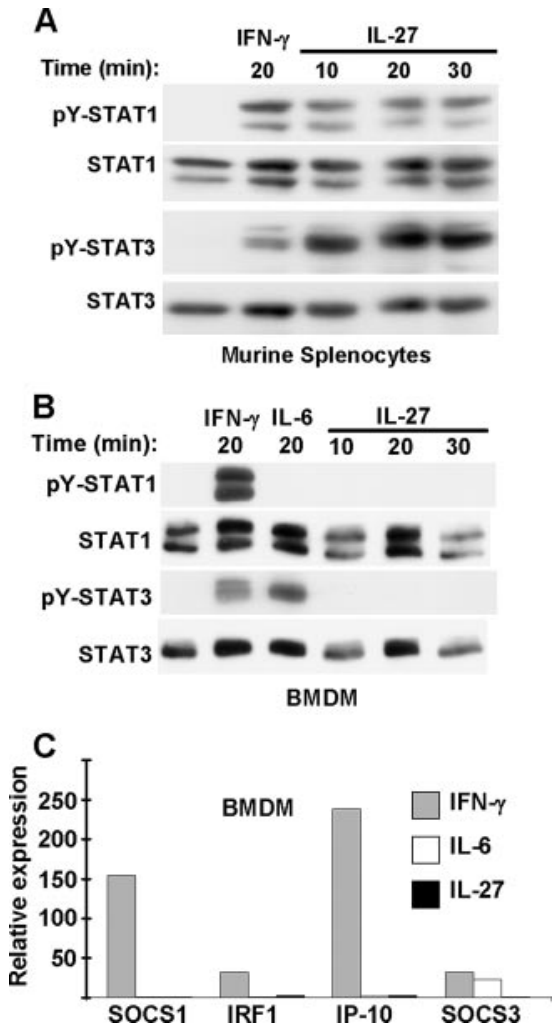

FIGURE 1. Nonactivated murine $\mathrm{M} \phi \mathrm{s}$ are minimally responsive to IL-27. $A$, Splenocytes obtained from C57BL/6J mice were stimulated with mIFN- $\gamma$ (100 U/ml) for $20 \mathrm{~min}$ or $\mathrm{mIL}-27(100 \mathrm{ng} / \mathrm{ml})$ for 10,20 , and $30 \mathrm{~min}$, and STAT1 and STAT3 tyrosine phosphorylation (pY) was measured by immunoblotting. $B$, BMDMs obtained from C57BL/6J mice were stimulated with $\mathrm{mIFN}-\gamma(100 \mathrm{U} / \mathrm{ml})$ or IL-6 $(50 \mathrm{ng} / \mathrm{ml})$ for $20 \mathrm{~min}$ or $\mathrm{mIL}-27(100 \mathrm{ng} / \mathrm{ml})$ for 10, 20, and $30 \mathrm{~min}$, and STAT1 and STAT3 tyrosine phosphorylation (pY) was measured by immunoblotting. $C$, BMDMs were cultured for $3 \mathrm{~h}$ with $\mathrm{mIFN}-\gamma(300 \mathrm{U} / \mathrm{ml})$, IL-6 $(50 \mathrm{ng} / \mathrm{ml})$, or $\mathrm{mIL}-27(100 \mathrm{ng} / \mathrm{ml})$ and mRNA was measured using real-time PCR and normalized relative to mGAPDH expression. Results are depicted as expression in cytokine-stimulated monocytes relative to unstimulated monocytes (set as 1) and the means $\pm \mathrm{SD}$ of triplicate determinants in a representative experiment of three independent experiments are shown; small SDs are not readily apparent because of large inductions. here as positive controls, activated STAT1 and STAT3 in murine M $\phi$ s (Fig. 1B). These results suggest that IL-27 activates STAT1 and STAT3 weakly in murine M $\phi$ s, below the limits of detection by the immunoblotting of whole cell lysates. Activation of STAT target genes is a very sensitive measure of STAT activation, and robust gene activation can be observed downstream of minimal or undetectable signals (24). Thus, we tested the effects of IL-27 stimulation on the expression of the STAT1-target genes SOCS1, IRF1, and $I P-10$ and the STAT3 target gene SOCS3. IL-27 stimulation had minimal, if any, effect on expression of these STAT target genes, in contrast to the robust activation of STAT1 target genes by IFN- $\gamma$ and SOCS3 by IL-6 (Fig. 1C). Overall, these results indicate that resting $\mathrm{mBMDMs}$ (and peritoneal $\mathrm{M} \phi \mathrm{s}$ ) are minimally responsive to IL-27, which is consistent with low or undetectable expression of the IL-27 receptor component WSX-1 in these cells (Ref. 3 and C. Hunter, unpublished observations).

\section{IL-27 activates predominantly STAT1-mediated responses in human monocytes and $M \phi$}

We next tested whether human monocytes and $\mathrm{M} \phi$ s were responsive to IL-27. IL-27 induced tyrosine phosphorylation of both STAT1 and STAT3 in freshly isolated human monocytes (Fig. 2, $A$ and $B$ ). IL-27 appeared to activate moderate levels of both of these STATs relative to the strong activation of STAT1 by IFN- $\gamma$ and the strong activation of STAT3 by IL-10 (Fig. 2, $A$ and $B$ ). Thus, at the time point tested (20 min after cytokine stimulation), IL-27 appeared to activate a balanced signal mediated by both STAT1 and STAT3 (Fig. 2, $A$ and $B$ ) that oppose each other's activity in many contexts $(22,23)$. In contrast, the activating cytokine IFN- $\gamma$ induced a STAT1-dominant signal and the suppressive cytokine IL-10 induced a STAT3-dominant signal (Fig. 2, A and $B$ ). Our next step was to investigate the induction of gene expression by IL-27. IL-27 strongly induced expression of the STAT1 target genes SOCS1, IRF1, CXCL10, and STAT1 comparably to the activation observed with saturating concentrations of IFN- $\gamma$ at several time points (Fig. 2, $C$ and $D$, and data not shown). IL-27 activated the expression of STAT1-dependent genes when de novo protein synthesis was blocked using cycloheximide (Fig. $2 D$ ), indicating that gene induction was direct and not dependent
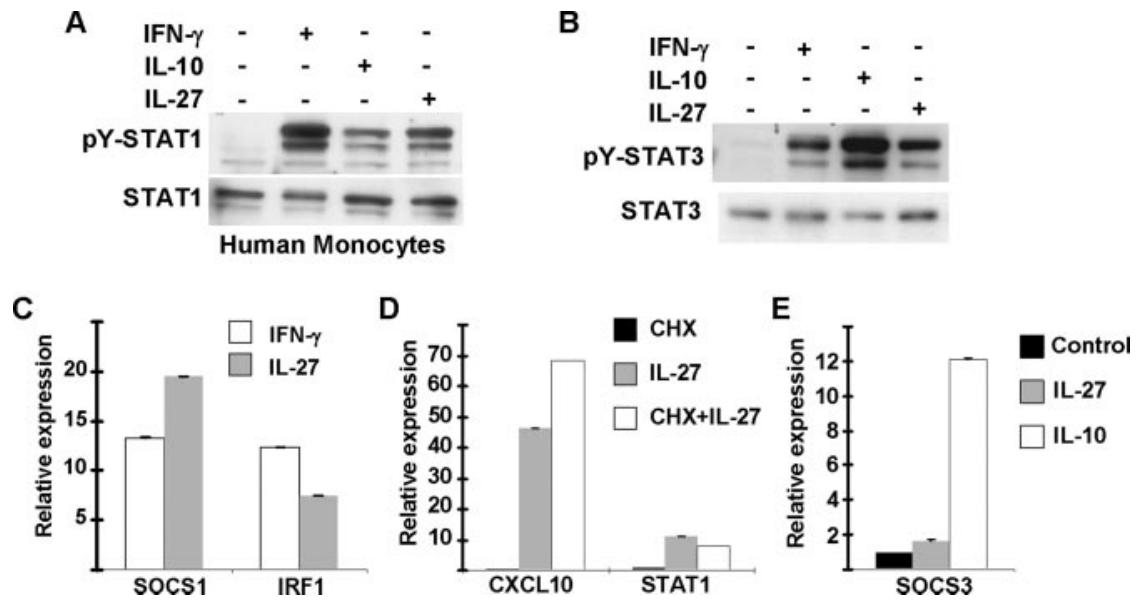

FIGURE 2. IL-27 activates STAT1 and STAT1 target genes in human monocytes. $A$ and $B$, Freshly isolated human monocytes were stimulated with IFN- $\gamma$ $(100 \mathrm{U} / \mathrm{ml})$, IL-10 (100 ng/ml), or IL-27 (100 ng/ml) for $15 \mathrm{~min}$ and STAT1 $(A)$ or STAT3 (B) tyrosine phosphorylation (pY) was measured by immunoblotting. Representative results of at least three independent experiments are shown. $C$, Monocytes were cultured for $3 \mathrm{~h}$ with IFN- $\gamma(100 \mathrm{U} / \mathrm{ml}) \mathrm{or} \mathrm{IL-27} \mathrm{(100} \mathrm{ng/ml)} \mathrm{and}$ mRNA expression was measured using real-time PCR and normalized relative to GAPDH expression. Representative results of at least 15 independent experiments are shown. $D$, Monocytes were cultured in the presence or absence of cycloheximide (CHX; $15 \mu \mathrm{g} / \mathrm{ml}$ ) for $30 \mathrm{~min}$, then stimulated with IL-27 (100 ng/ml) for $3 \mathrm{~h}$, and mRNA was measured using real-time PCR. E, Monocytes were cultured for $3 \mathrm{~h}$ with IL-10 (100 ng/ml) or IL-27 (100 ng/ml) and mRNA was measured using real-time PCR. For $D$ and $E$, representative results from at least three independent experiments are shown. Results are depicted as expression in cytokinestimulated monocytes relative to unstimulated monocytes (set as 1) and the means \pm SD of triplicate determinants in a representative experiment are shown. 
on synthesis of other cytokines. In contrast to the robust activation of STAT1 target genes, the induction of the STAT3 target gene SOCS3 in response to saturating concentrations of IL-27 was very modest and markedly lower compared with induction by IL-10 (Fig. 2E). The effects of IL-27 on STAT activation and gene expression were highly reproducible and were observed in $>15$ experiments with monocytes derived from different blood donors. These results suggest that IL-27 has a STAT1-dominant (IFN- $\gamma$ like) effect on gene activation in human monocytes despite the simultaneous activation of STAT3.

The duration of STAT activation is an important determinant of downstream gene expression, such that sustained activation of STATs is more effective in inducing gene expression than transient activation of comparable magnitude $(37,38)$. Therefore, we investigated the kinetics of STAT activation downstream of the IL-27 receptor. In these experiments we compared IL-27 to IL-6, which also activates both STAT1 and STAT3 (33). The IL-27 and IL-6 receptors both use the gp130 signaling subunit that activates STAT1 and STAT3 (Fig. 3A). In addition, the IL-27 receptor contains the WSX-1 subunit that confers ligand specificity and activates STAT1 (13). Comparable levels of STAT1 tyrosine phosphorylation were induced after 15 min of stimulation with IL-6 and IL-27 (Fig. 3A). IL-6-induced STAT1 tyrosine phosphorylation decayed rapidly and returned almost to baseline $30 \mathrm{~min}$ after the addition of IL-6. In contrast, IL-27 induced sustained STAT1 tyrosine phosphorylation that was still elevated $120 \mathrm{~min}$ after the addition of IL-27 (Fig. 3A). Activation of STAT3 by IL-6 and IL-27, mediated in both cases by gp130, followed similar kinetics (Fig. 3A). Subsequently, we tested whether the substantial difference in the duration of STAT1 activation by IL-27 and IL-6 was associated with differential induction of STAT1 target genes. The STAT1 target genes SOCS1, IRF1, and CXCL10 were induced by IL-27 (Fig. 3B), consistent with our previous observations (Fig. 2, $C$ and $D$ ). In contrast, IL-6 had a minimal effect on the expression of STAT1 target genes (Fig. 3B). These results suggest that WSX-1 contributes to a sustained STAT1 response that is transduced into effective activation of downstream genes.

\section{IL-27 primes monocytes for enhanced TLR responses by a STAT1-dependent mechanism}

We wished to investigate the functional consequences of IL-27 signaling on inflammatory cytokine production by monocytes. IFN- $\gamma$ enhances TLR-induced inflammatory cytokine production, whereas IL-10 suppresses cytokine production by a STAT3-dependent mechanism $(31,32)$. We tested whether IL-27 would enhance or suppress TLR-induced cytokine production in primary human monocytes. We cultured human monocytes in the presence or absence of IL-27, and then stimulated cells with TLR ligands and measured cytokine production using ELISA. As expected, stimulation of monocytes with $\mathrm{Pam}_{3} \mathrm{Cys}$ (TLR2 ligand) induced the production of the proinflammatory cytokines TNF- $\alpha$ and IL-6 (Fig. 4A, open bars). Cells that had been pretreated with IL-27 produced substantially higher levels of TNF- $\alpha$ and IL- 6 in response to $\mathrm{Pam}_{3}$ Cys (Fig. $4 A$, gray bars). In parallel, IL-27 pretreatment augmented TLR2-induced accumulation of TNF- $\alpha$ and IL-6 mRNA (Fig. 4B), and similar results were obtained when IL-1 $\beta$ and IL-12 p40 mRNA were measured (data not shown). IL-27 also augmented inflammatory cytokine production in response to the TLR4 ligand LPS (Fig. 4, $C$ and $D$ ) and the TLR7/8 ligand CL097 (data not shown).

The priming effects of IL-27 on TLR-induced inflammatory cytokine production are similar to those exerted by IFN- $\gamma$, a strong activator of STAT1 (31). Therefore, we wished to test whether augmented cytokine production was dependent on
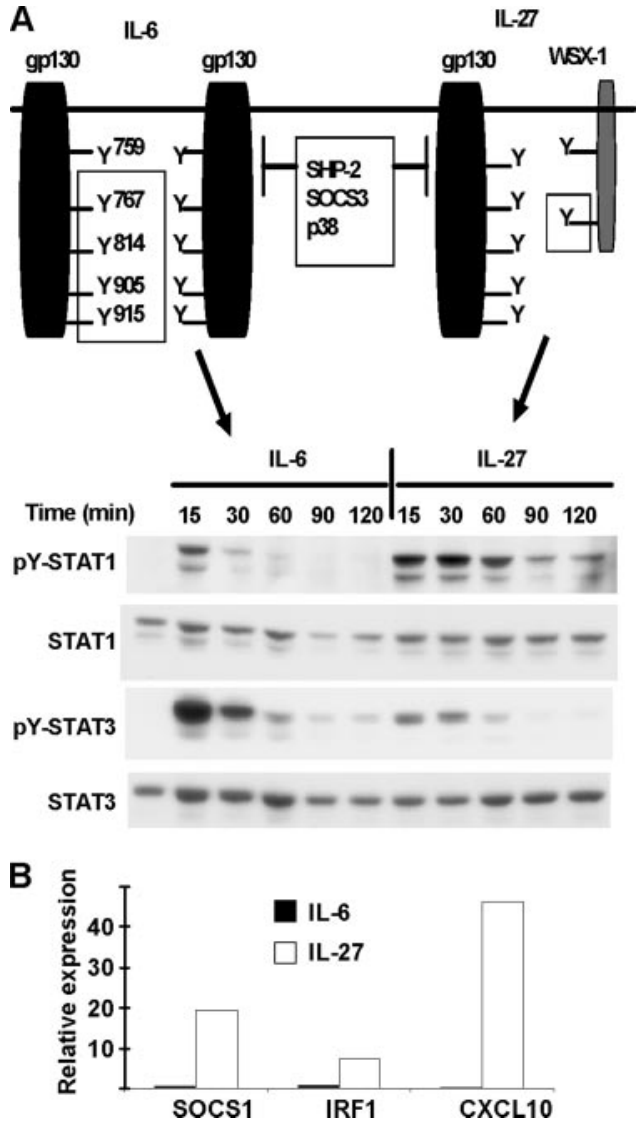

FIGURE 3. Prolonged activation of STAT1 correlates with induction of STAT1 target genes downstream of the IL-27 receptor. A, IL-6 signals via gp130 homodimers whereas IL-27 signals via WSX-1 and gp130 heterodimers. gp130 is subject to negative regulation by SOCS3 and the phosphatase SHP2 via tyrosine 759 (Y759) and by p38. gp130 tyrosines 767, 814, 905, and 915 recruit STAT3, tyrosines 905 and 915 also recruit STAT1, and the membrane-distal tyrosine of WSX-1 recruits STAT1. Monocytes were cultured with IL-6 $(50 \mathrm{mg} / \mathrm{ml})$ plus IL-6R $\alpha(100 \mathrm{ng} / \mathrm{ml})$ or IL-27 $(100 \mathrm{ng} / \mathrm{ml})$ for the indicated times and STAT1 and STAT3 tyrosine phosphorylation (pY) was measured by immunoblotting. Representative results of two independent experiments are shown. $B$, Monocytes were cultured for $3 \mathrm{~h}$ with IL-6 $(50 \mathrm{mg} / \mathrm{ml})$ plus IL-6R $\alpha(100 \mathrm{ng} / \mathrm{ml})$ or IL-27 (100 ng/ml), and mRNA was measured using real-time PCR. Representative results from at least three independent experiments are shown. Results are depicted as expression in cytokine-stimulated monocytes relative to unstimulated monocytes (set as 1 ) and the means \pm SD of triplicate determinants in a representative experiment are shown.

STAT1. We approached this question by using human monocytic THP-1 cells that had been stably transduced with lentiviral vectors encoding short hairpin RNA (shRNA) that effectively silences STAT1 expression (or control shRNA) as previously described (27). Consistent with our observations using primary monocytes, THP-1 cells transduced with control shRNA produced increased levels of TLR2-induced IL-6 after priming with IL-27 (Fig. 4E). In contrast, this priming effect on TLR2induced IL-6 production was abolished in cells in which STAT1 expression was silenced (Fig. 4E). These results show that IL-27 augments TLR-induced inflammatory cytokine production by a STAT1-dependent mechanism.

\section{$I L-27$ modulates $I L-10$ function and suppresses $I L-10$ production}

Effective priming and activation of monocytes involves overcoming suppression by regulatory factors such as IL-10 and 

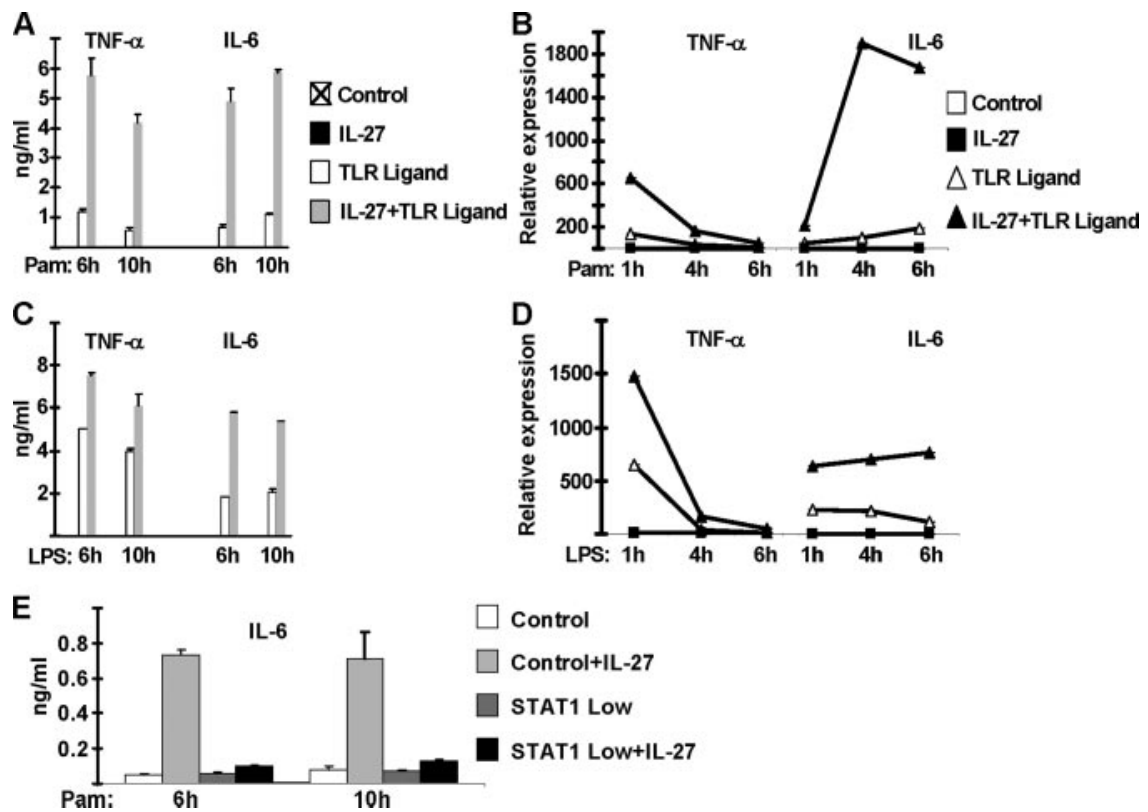

FIGURE 4. IL-27 primes monocytes for enhanced TLR responses by a STAT1-dependent mechanism. $A-D$, Monocytes were cultured with M-CSF $(10 \mathrm{ng} / \mathrm{ml})$ in the presence or absence of IL-27 (100 ng/ml) for $24 \mathrm{~h}$ and stimulated for the indicated times with Pam ${ }_{3} \mathrm{Cys}(\mathrm{Pam} ; 10 \mathrm{ng} / \mathrm{ml})(A$ and $B)$ or LPS $(10 \mathrm{ng} / \mathrm{ml})(C$ and $D)$. TNF- $\alpha$ and IL-6 protein were measured in culture supernatants using ELISA $(A$ and $C)$ and mRNA was measured using real time PCR $(B$ and $D)$. Representative results of at least five independent experiments are shown. For $B$ and $D$, results are depicted as expression in cytokine-stimulated monocytes relative to unstimulated monocytes (set as 1) and the means \pm SD of triplicate determinants in a representative experiment are shown. E, THP-1 monocytic cells transduced with lentiviral vectors encoding control or STAT1-specific shRNA interference cells (STAT1 Low) were cultured in the presence or absence of IL-27 ( $200 \mathrm{ng} / \mathrm{ml}$ ) for $24 \mathrm{~h}$, stimulated with Pam ${ }_{3}$ Cys, and IL-6 in culture supernatants was measured using ELISA. Representative results of at least three independent experiments are shown.

TGF- $\beta$ (39). As IL-10 is a key deactivator of monocytes $(22$, 32 ), we investigated the effects of IL-27 on IL-10 signaling and function. The suppressive effects of IL-10 are mediated by STAT3, and we tested the effects of IL-27 on IL-10-induced STAT activation. IL-10 induced tyrosine phosphorylation of STAT3 as expected, and this induction was not affected by IL-27 (Fig. 5A). As predicted based on the mRNA results (Fig. $2 D$ ), IL-27 strongly increased STAT1 protein expression (Fig. $5 A$ ). Concomitant with increased STAT1 expression, IL-10 in- duced increased levels of STAT1 tyrosine phosphorylation in monocytes that had been primed with IL-27 (Fig. 5A). Because STAT1 can suppress the expression of STAT3 target genes (27), we tested the effects of IL-27 on IL-10-induced gene expression. Interestingly, IL-27 attenuated the induction of SOCS3, PGDH, DEPP, CD163, and CR1 genes by IL-10 (Fig. $5 B$ ), suggesting that IL-27 may attenuate IL-10 function, including suppressive functions mediated by SOCS3 and PGDH $(38,40)$.

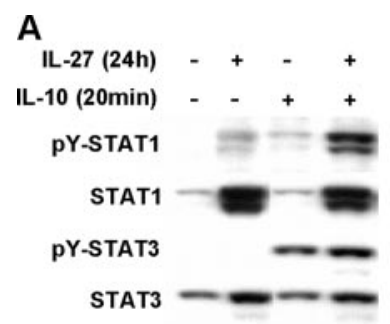

B
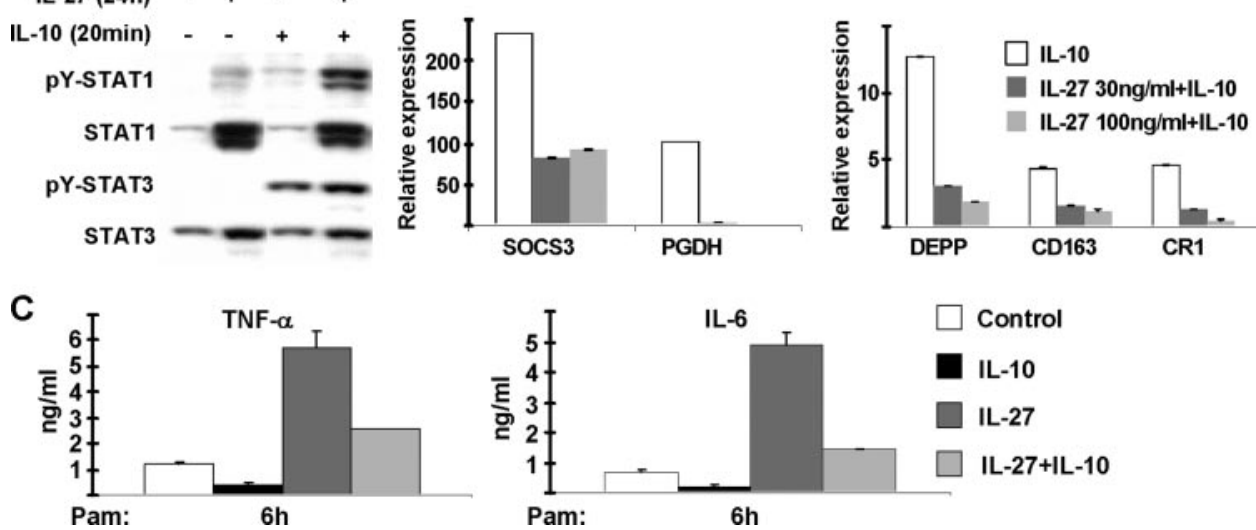

FIGURE 5. IL-27 modulates IL-10 signaling and function. Monocytes were cultured with M-CSF (10 ng/ml) in the presence or absence of IL- 27 ( 30 or $100 \mathrm{ng} / \mathrm{ml})$ for $24 \mathrm{~h}$. A, Control and IL-27-primed cells were stimulated with or without IL-10 (20 ng/ml) for 20 min and cell lysates were analyzed by immunoblotting. PY, Tyrosine phosphorylation. B, Control and IL-27-primed cells were stimulated with IL-10 (20 ng/ml) for $3 \mathrm{~h}$ and SOCS3, PGDH, DEPP, CD163, and CR1 mRNA were measured using real-time PCR and normalized relative to GAPDH expression. $C$, Control and IL-27-primed cells were cultured for $3 \mathrm{~h}$ in the presence or absence of IL-10 (20 ng/ml) and then stimulated for $6 \mathrm{~h}$ with Pam ${ }_{3}$ Cys (Pam). Production of TNF- $\alpha$ and IL-6 protein was measured in the culture supernatants using ELISA. Representative results from at least three independent experiments are shown. For $B$, results are depicted as expression in cytokine-stimulated monocytes relative to unstimulated monocytes (set as 1), and the means \pm SD of triplicate determinants in a representative experiment are shown. 
FIGURE 6. IL-27 suppresses IL-10 production from human monocytes. Monocytes were cultured with M-CSF $(10 \mathrm{ng} / \mathrm{ml})$ in the presence or absence of IL-27 (100 ng/ml) for $24 \mathrm{~h}$, stimulated for the indicated times with $\mathrm{Pam}_{3} \mathrm{Cys}$ $(\mathrm{Pam} ; 10 \mathrm{ng} / \mathrm{ml})(A$ and $B)$, LPS $(10 \mathrm{ng} / \mathrm{ml})(C$ and $D$ ) or zymosan (Zym; $40 \mu \mathrm{g} / \mathrm{ml})(E)$. IL-10 protein in culture supernatants was measured using ELISA, and IL-10 mRNA was measured using real time PCR. Representative results from at least four independent experiments are shown. For $B$ and $D$, results are depicted as expression in cytokine-stimulated monocytes relative to unstimulated monocytes (set as 1) and the means $\pm \mathrm{SD}$ of triplicate determinants in a representative experiment are shown.
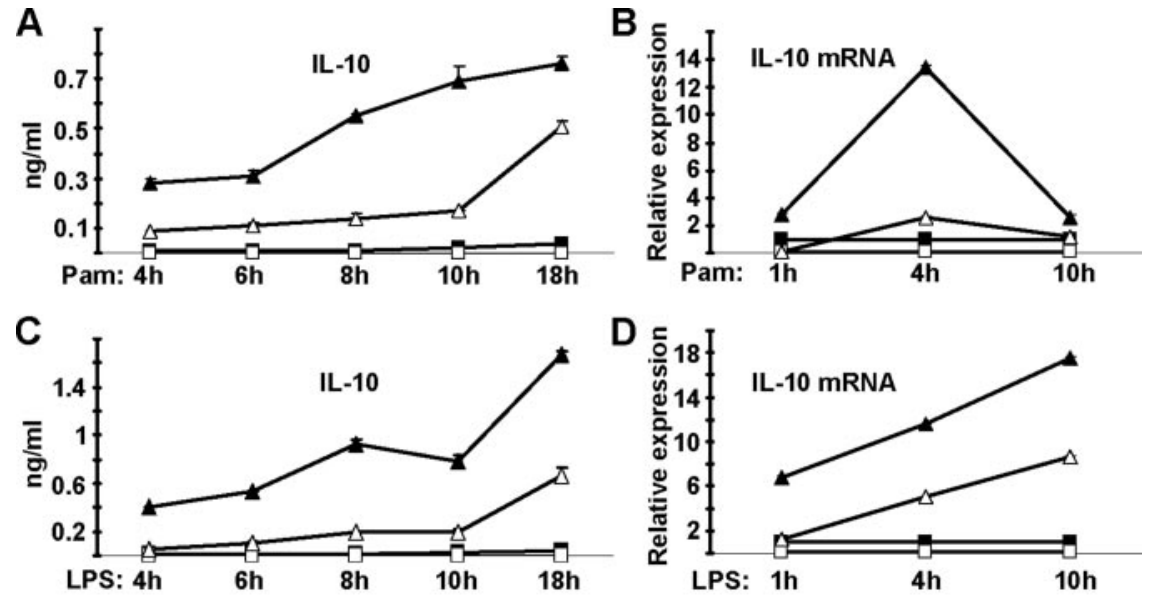

E

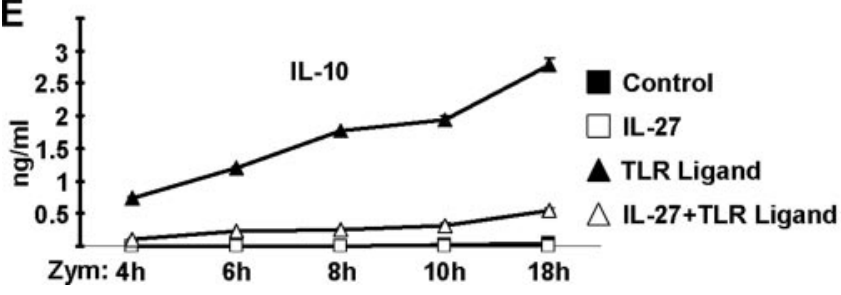

We then tested whether IL-27 could attenuate a key suppressive function of IL-10, namely IL-10-mediated down-regulation of inflammatory cytokine production. As expected, IL-10 suppressed $\mathrm{Pam}_{3}$ Cys-induced production of TNF- $\alpha$ and IL-6 (Fig. $5 C$ ). In accord with our previous results, IL-27 augmented TLR-induced TNF- $\alpha$ and IL-6 production (Fig. 5C). This augmented cytokine production was still effectively suppressed by IL-10. However, even though IL-10 still maintained suppressive effects on cytokine production in IL-27-primed monocytes, monocytes that were treated with both IL-27 and IL-10 produced relatively high levels of TNF- $\alpha$ and IL-6 (Fig. 5C, light gray bars), comparable to or higher than cytokine levels produced by control monocytes stimulated with TLR ligands in the absence of IL-10 (Fig. 5C, open bars). Similar results were obtained when monocytes were stimulated with LPS (data not shown). These results make two points: 1 ) when the total monocyte output of TNF- $\alpha$ and IL-6 are considered, the proinflammatory effects of IL-27 dominate over the suppressive effects of IL-10; and 2) despite these quantitative differences, IL-10 retains its ability to suppress TLR-induced cytokine production in the presence of IL-27.

Because IL-27 did not abrogate the suppressive effects of IL-10 on cytokine production, we next addressed whether IL-27 regulated TLR-induced IL-10 production, which serves an important feedback inhibitory and homeostatic function (32). Treatment of monocytes with IL-27 resulted in a striking inhibition of $\mathrm{Pam}_{3} \mathrm{Cys}$ induction of IL-10 protein and mRNA (Fig. 6, $A$ and $B$ ). IL-27 had similar inhibitory effects on the induction of IL-10 by LPS (Fig. 6 , $C$ and $D$ ) and by zymosan (Fig. $6 E$ ), in which case IL-10 production is mainly dependent on dectin-1, which signals by tyrosine kinase pathways (41). Thus, in striking contrast to $\mathrm{T}$ cells where IL-27 induces IL-10 production (9-11), IL-27 broadly suppresses IL-10 production in human monocytes. Collectively, the results indicate that disruption of IL-10-mediated feedback inhibition and suppression is a component of the activating function of IL-27 on human monocytes.
Activation of monocytes by IL-27 is abrogated by TLRs via a p38-dependent mechanism

The activating effects of IL-27 on fresh human monocytes that we have described stand in stark contrast to reports that document a predominantly suppressive function of IL-27 on acquired immunity at later stages of immune responses $(6-8,12,16-19,36)$. We reasoned that IL-27 may prime the first steps of innate immune responses, similar to early activation of naive $\mathrm{T}$ cells $(2-4,16$, 42-44), but that these priming functions may be blocked as the immune response evolves and IL-27 function shifts to a suppressive mode. We tested this notion by investigating whether activated monocytes became refractory to the effects of IL-27. Strikingly, activation of primary human monocytes with LPS strongly inhibited IL-27-induced STAT1 activation over a sustained time period (Fig. 7A). Concomitant with the inhibition of signaling, LPS inhibited IL-27-induced activation of the STAT1 target genes IRF1, STAT1, CXCL10, and CXCL9 (Fig. 7B); the time frame of these experiments is short, such that the LPS-mediated induction of $C X C L 9$ and $C X C L 10$ that occurs indirectly via autocrine IFN- $\beta$ is not readily detectable. The IL-27 receptor is comprised of gp130 and WSX-1 subunits (13), and we and others have shown that gp130-mediated signaling is strongly inhibited by TLRs via a rapidly induced mechanism that is dependent on p38 and independent of de novo protein synthesis (45-51). Interestingly, inhibition of IL-27 signaling was induced rapidly, within 30 min of TLR stimulation (Fig. 7A), and was independent of de novo RNA or protein synthesis (Fig. 7C). In addition, LPS-induced suppression was reversed when the p38 inhibitor SB203580 was used (Fig. 7D) and, thus, was dependent on the activation of p38. As LPS inhibits IL-6 and IL-27 signaling under the same conditions (Fig. 7E) by a similar p38-dependent but RNA and protein synthesis-independent mechanism (45-51), these results suggest that TLRs inhibit IL-27 signaling in part by targeting the gp130 receptor subunit. This raises the possibility that although IL-27 and IFN- $\gamma$ share STAT1activating properties, the functions of these cytokines may be differentially regulated by TLRs. This notion was supported by the 
A

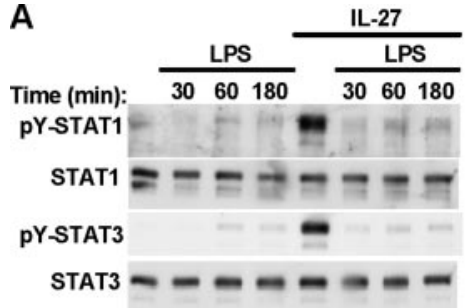

C
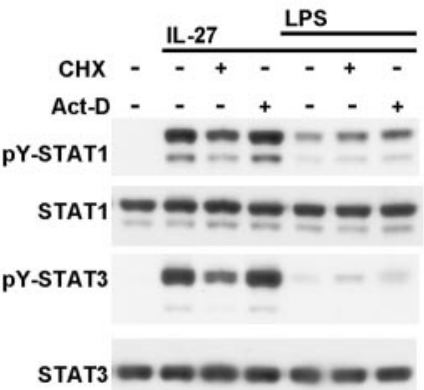

E

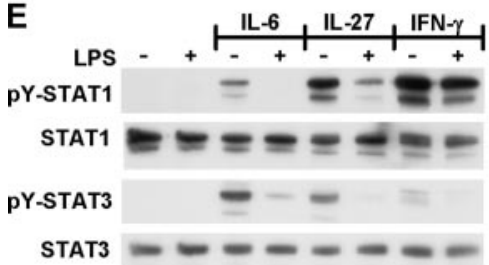

B

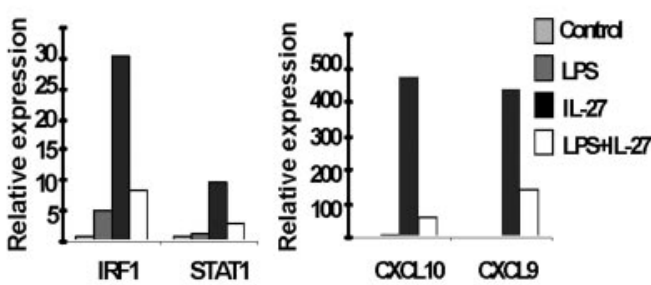

D

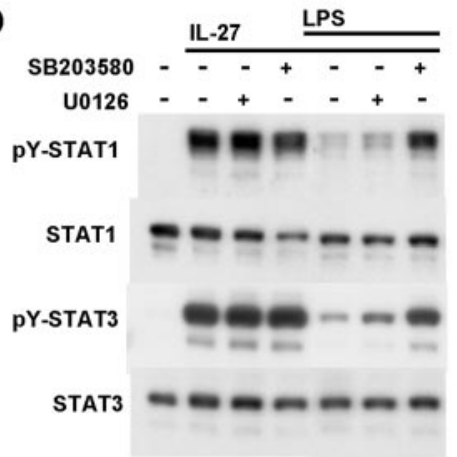

$\mathbf{F}$

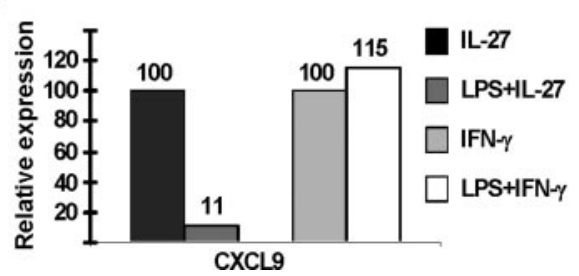

FIGURE 7. Activation of monocytes by IL-27 is abrogated by TLRs by a p38-dependent mechanism. A, Monocytes were cultured in the presence or absence of LPS $(10 \mathrm{ng} / \mathrm{ml})$ for the indicated times, stimulated with IL-27 (100 ng/ml) and STAT1, and STAT3 tyrosine phosphorylation (pY) was measured by immunoblotting. Representative results of at least five independent experiments are shown. $B$, Monocytes were cultured in the presence or absence of LPS for $30 \mathrm{~min}$, stimulated with IL-27 for $3 \mathrm{~h}$, and IRF1, STAT1, CXCL10, and CXCL9 mRNA were measured using real-time PCR and normalized relative to GAPDH expression. Representative results of at least three independent experiments are shown. $C$, Monocytes were cultured in the presence or absence of cycloheximide (CHX; $15 \mu \mathrm{g} / \mathrm{ml}$ ) or actinomycin D (Act-D; $5 \mu \mathrm{g} / \mathrm{ml}$ ) for 30 min before stimulation with LPS (30 min) and then with IL-27 (20 min) as in A. STAT1 and STAT3 tyrosine phosphorylation (pY) was measured by immunoblotting. $D$, Monocytes were cultured in the presence or absence of SB203580 (p38 inhibitor; $10 \mu \mathrm{M}$ ) or U0126 (MEK inhibitor; $10 \mu \mathrm{M}$ ) before LPS and IL-27 stimulation as in $C$. For $C$ and $D$, representative results of at least two independent experiments are shown. E, Monocytes were cultured in the presence $(+)$ or absence ( - ) of LPS for 30 min and then stimulated with IL-6 (50 ng/ml) plus IL-6R $\alpha$ (100 ng/ml), IL-27 (100 ng/ml), or IFN- $\gamma(100$ U/ml) for $20 \mathrm{~min}$ and STAT1 and STAT3 activation was measured by immunoblotting. $F$, Monocytes were cultured in the presence or absence of LPS for 30 min, stimulated with IL- 27 or IFN- $\gamma$ for $3 \mathrm{~h}$ and $\mathrm{mRNA}$ was measured using real-time PCR. Expression of CXCL9 mRNA in LPS-pretreated cells relative to cells stimulated with cytokines alone (each set as 100) is shown. For $E$ and $F$, representative results from at least three independent experiments are shown. For $B$ and $F$, results are depicted as expression in cytokine-stimulated monocytes relative to unstimulated monocytes (set as 1), and the means \pm SD of triplicate determinants in a representative experiment are shown.

finding that, in contrast to the inhibition of IL-27 responses, IFN$\gamma$-induced activation of STAT1 and downstream expression of STAT1 target genes were preserved in LPS-activated monocytes (Fig. 7, $E$ and $F$ ). Collectively, the results show that IL-27 function in human monocytes is tightly regulated, and the activating functions of IL-27 that can promote the earliest steps of innate responses are rapidly attenuated as monocytes become activated and the immune response matures.

\section{Discussion}

Emerging paradigms about the role of IL-27 in the orchestration of immune responses suggest that IL-27 is pleiotropic and has opposite activating or suppressing effects dependent on timing and context. Recent work suggests a predominant suppressive role for IL-27 that dampens acquired immunity and attenuates the later phases of immune activation by suppressing T cells. We found that IL-27 has strong activating effects on human monocytes, leading to IFN- $\gamma$-like activation of STAT1 target genes, increased production of chemokines and inflammatory cytokines, and attenuated IL-10 function and production. These findings suggest an activating function of IL-27 on human innate immunity that contrasts with its suppressive effects on acquired immunity. However, this activating function of IL-27 is transient, as IL-27 signaling was inactivated by TLR ligands via a rapidly induced p38-dependent inhibitory mechanism. Inhibition of IL-27 signaling in M $\phi$ s provides a mechanism by which IL-27 transitions from early activating effects on innate immunity to switch to predominantly suppressive effects on acquired immunity.

The activation of human monocytes by IL-27 has similarities but also significant differences from activation by IFN- $\gamma$, the prototypic $\mathrm{M} \phi$-activating cytokine. Many of these similar actions can be explained on the basis of STAT1 activation, which leads to expression of canonical inflammatory STAT1 target genes such as chemokines (27) and to suppression of IL-10 production in myeloid cells in vitro (39) and in vivo (52). Interestingly, STAT1 appears to have the opposite effect on IL-10 production in T cells, where IL-27 induces IL-10 production by a STAT1-dependent (and also STAT3-dependent) mechanism $(10,11)$. We have also 
shown for the first time that priming for increased TLR-induced inflammatory cytokine production is dependent on STAT1; it is likely that priming by IFN- $\gamma$ depends on STAT1, similar to priming by IL-27 (Fig. 4). Interesting differences between IL-27 and IFN- $\gamma$ include cellular source, regulation of production, regulation of cellular responses to these cytokines, and different effects on acquired immunity. IL-27 is produced mainly by APCs in response to microbial products. IFN- $\gamma$ is also produced by NK and NKT cells that participate in innate immunity; however, Ag-specific T cells are a major source of IFN- $\gamma$, and thus IFN- $\gamma$ production can be finely regulated in an Ag-specific manner. In contrast, $\mathrm{M} \phi$ responses to IL-27 are more tightly regulated than responses to IFN- $\gamma$, as TLR stimulation inhibited IL-27 but not IFN- $\gamma$ signaling. Thus it appears that IL-27 and IFN- $\gamma$ have similar functions on human monocytes, but the expression of and signaling by these cytokines are differentially regulated to tune $\mathrm{M} \phi$ responses to be appropriate for the environment.

IL-27 uses gp130 that is also used by IL-6 family cytokines; gp130 activates both STAT3 and STAT1 with a tendency to activate a STAT3-dominant response $(53,54)$. In contrast to IL-6 that activated STAT1 transiently in monocytes, IL-27 activated sustained tyrosine phosphorylation of STAT1 that correlated with a much stronger IL-27 induction of STAT1 target gene expression (Fig. 3). The duration of STAT activation has been associated with the magnitude of target gene expression $(28,37,38)$, and the sustained pattern of STAT1 activation by IL-27 is likely conferred by WSX-1, because the activation of STATs by gp130 was transient in monocytes (Fig. 3) and WSX-1 has been shown to activate STAT1 in other systems (13). Interestingly, our group and others have shown that gp130 signaling is highly regulated and is targeted for inhibition by multiple mechanisms, including SOCS3 and Src homology domain 2-containing phosphatase (SHP-2), that bind to tyrosine 759 in the gp130 cytoplasmic domain and by a rapidly acting p38-dependent pathway $(45-51,55,56)$ that likely induces receptor internalization (Ref. 57 and G. Kalliolias, unpublished observations) (Fig. 3A). Thus, gp130 functions not just as a signal transducer but as a target for inhibition that confers suppression on signaling by receptor subunits such as WSX-1, with which it associates. In contrast, activation of STAT1 by IFN- $\gamma$ is resistant to inhibition by TLR-induced pathways. The association of WSX-1 with gp130 thus provides a mechanism by which STAT1 can be strongly activated but also becomes subject to inhibition by factors that activate $\mathrm{p} 38$.

Our results suggest differences between human monocytes that are strongly activated by IL-27 and murine BMDMs and peritoneal $\mathrm{M} \phi$ s that have minimal responsiveness in our hands (Fig. 1), consistent with low WSX-1 expression in these M $\phi$ populations (Ref. 3 and C. Hunter, unpublished observations). IL-27 function is context dependent and it is possible that IL-27 activates STAT1 in murine myeloid populations more directly comparable (but difficult to access experimentally) to the human monocytes studied here. Other laboratories have actually detected modest direct suppressive effects of IL-27, accompanied by STAT3 activation, in activated murine $\mathrm{M} \phi \mathrm{s}$ and dendritic cells cultured under different conditions than those used in our study $(8,21)$. It is not yet clear whether these differences between the activation of human monocytes and the modest suppression of murine myeloid cells reflect differences related to cell differentiation and activation state or represent a qualitative species difference, possibly related to differential relative activation of STAT3 vs STAT1 by murine and human IL-27 receptors. Alternatively, these differences may be related to differences in WSX-1 expression, which is regulated by cytokines $(58,59)$ in different monocyte/ $\mathrm{M} \phi$ populations, or to differential targeting and modulation of gp130 signaling. To date we have been unsuccessful in detecting activating responses to IL-27 in several murine $\mathrm{M} \phi$ populations while finding that human cells maintain IL-27 responsiveness during differentiation into $\mathrm{M} \phi$ s during culture with M-CSF (G. Kalliolias, unpublished observations). Future work comparing the expression and signaling of human vs murine WSX-1 will help resolve these issues.

Our results suggest a temporal regulation of monocyte/M $\phi$ responses to IL-27 during the course of an immune response. In this model, during the early phases of innate immune responses circulating monocytes or recent monocytic emigrants into inflammatory sites are strongly activated by systemic or paracrine IL-27, respectively. If monocytes encounter microbes or their products at sites of infection, they will start to produce IL-27 but at the same time become refractory to its effects. Thus the amplifying effects and potential toxicity of autocrine IL-27 are avoided, and IL-27 can act in a paracrine manner, eventually to suppress lymphocyte responses. This model postulates that the pleiotropic biological effects of IL-27 are in part mediated by induced changes in IL-27 signaling in monocytes. This mechanism of regulation is similar to the reprogramming of the pattern of STAT activation that occurs in $\mathrm{T}$ cells during their activation (16) and has been suggested to alter $\mathrm{T}$ cell responses to IL-27 in a context-dependent manner. Overall, our observations extend our understanding of the bidirectional and context-dependent role of IL-27 in the orchestration of immune responses.

\section{Acknowledgments}

We thank Jong Dae Ji and Kyung-Hyun Park-Min for critical reading of the manuscript. We thank Simi Ahmed and Zhimei Du for some of the IFN- $\gamma$ experiments.

\section{Disclosures}

The authors have no financial conflict of interest.

\section{References}

1. Kastelein, R. A., C. A. Hunter, and D. J. Cua. 2007. Discovery and biology of IL-23 and IL-27: related but functionally distinct regulators of inflammation. Annu. Rev. Immunol. 25: 221-242.

2. Chen, Q., N. Ghilardi, H. Wang, T. Baker, M. H. Xie, A. Gurney, I. S. Grewal, and F. J. de Sauvage. 2000. Development of Th1-type immune responses requires the type I cytokine receptor TCCR. Nature 407: 916-920.

3. Yoshida, H., S. Hamano, G. Senaldi, T. Covey, R. Faggioni, S. Mu, M. Xia, A. C. Wakeham, H. Nishina, J. Potter, et al. 2001. WSX-1 is required for the initiation of Th1 responses and resistance to L. major infection. Immunity 15: $569-578$.

4. Pflanz, S., J. C. Timans, J. Cheung, R. Rosales, H. Kanzler, J. Gilbert, L. Hibbert, T. Churakova, M. Travis, E. Vaisberg, et al. 2002. IL-27, a heterodimeric cytokine composed of EBI3 and $\mathrm{p} 28$ protein, induces proliferation of naive $\mathrm{CD} 4^{+} \mathrm{T}$ cells. Immunity 16: 779-790

5. Hunter, C. A., A. Villarino, D. Artis, and P. Scott. 2004. The role of IL-27 in the development of T-cell responses during parasitic infections. Immunol. Rev. 202: $106-114$.

6. Villarino, A., L. Hibbert, L. Lieberman, E. Wilson, T. Mak, H. Yoshida, R. A. Kastelein, C. Saris, and C. A. Hunter. 2003. The IL-27R (WSX-1) is required to suppress $\mathrm{T}$ cell hyperactivity during infection. Immunity 19: 645-655.

7. Hamano, S., K. Himeno, Y. Miyazaki, K. Ishii, A. Yamanaka, A. Takeda, M. Zhang, H. Hisaeda, T. W. Mak, A. Yoshimura, and H. Yoshida. 2003. WSX-1 is required for resistance to Trypanosoma cruzi infection by regulation of proinflammatory cytokine production. Immunity 19: 657-667.

8. Holscher, C., A. Holscher, D. Ruckerl, T. Yoshimoto, H. Yoshida, T. Mak, C. Saris, and S. Ehlers. 2005. The IL-27 receptor chain WSX-1 differentially regulates antibacterial immunity and survival during experimental tuberculosis. J. Immunol. 174: 3534-3544.

9. Awasthi, A., Y. Carrier, J. P. Peron, E. Bettelli, M. Kamanaka, R. A. Flavell, V. K. Kuchroo, M. Oukka, and H. L. Weiner. 2007. A dominant function for interleukin 27 in generating interleukin 10-producing anti-inflammatory $\mathrm{T}$ cells. Nat. Immunol. 8: 1380-1389.

10. Fitzgerald, D. C., G. X. Zhang, M. El-Behi, Z. Fonseca-Kelly, H. Li, S. Yu, C. J. Saris, B. Gran, B. Ciric, and A. Rostami. 2007. Suppression of autoimmune inflammation of the central nervous system by interleukin 10 secreted by interleukin 27-stimulated T cells. Nat. Immunol. 8: 1372-1379.

11. Stumhofer, J. S., J. S. Silver, A. Laurence, P. M. Porrett, T. H. Harris, L. A. Turka, M. Ernst, C. J. Saris, J. J. O'Shea, and C. A. Hunter. 2007. Interleukins 27 and 6 induce STAT3-mediated T cell production of interleukin 10. Nat. Immunol. 8: 1363-1371. 
12. Batten, M., and N. Ghilardi. 2007. The biology and therapeutic potential of interleukin 27. J. Mol. Med. 85: 661-672.

13. Pflanz, S., L. Hibbert, J. Mattson, R. Rosales, E. Vaisberg, J. F. Bazan, J. H. Phillips, T. K. McClanahan, R. de Waal Malefyt, and R. A. Kastelein. 2004. WSX-1 and glycoprotein 130 constitute a signal-transducing receptor for IL-27. J. Immunol. 172: 2225-2231.

14. Hibbert, L., S. Pflanz, R. De Waal Malefyt, and R. A. Kastelein. 2003. IL-27 and IFN- $\alpha$ signal via Stat 1 and Stat 3 and induce T-Bet and IL-12R $\beta 2$ in naive T cells. J. Interferon Cytokine Res. 23: 513-522.

15. Lucas, S., N. Ghilardi, J. Li, and F. J. de Sauvage. 2003. IL-27 regulates IL-12 responsiveness of naive $\mathrm{CD} 4^{+} \mathrm{T}$ cells through Stat 1-dependent and -independent mechanisms. Proc. Natl. Acad. Sci. USA 100: 15047-15052.

16. Yoshimura, T., A. Takeda, S. Hamano, Y. Miyazaki, I. Kinjyo, T. Ishibashi, A. Yoshimura, and H. Yoshida. 2006. Two-sided roles of IL-27: induction of Th1 differentiation on naive $\mathrm{CD}^{+} \mathrm{T}$ cells versus suppression of proinflammatory cytokine production including IL-23-induced IL-17 on activated CD4 ${ }^{+} \mathrm{T}$ cells partially through STAT3-dependent mechanism. J. Immunol. 177: 5377-5385.

17. Batten, M., J. Li, S. Yi, N. M. Kljavin, D. M. Danilenko, S. Lucas, J. Lee, F. J. de Sauvage, and N. Ghilardi. 2006. Interleukin 27 limits autoimmune encephalomyelitis by suppressing the development of interleukin 17-producing $\mathrm{T}$ cells. Nat. Immunol. 7: 929-936.

18. Stumhofer, J. S., A. Laurence, E. H. Wilson, E. Huang, C. M. Tato, L. M. Johnson, A. V. Villarino, Q. Huang, A. Yoshimura, D. Sehy, et al. 2006. Interleukin 27 negatively regulates the development of interleukin 17-producing $\mathrm{T}$ helper cells during chronic inflammation of the central nervous system. Nat. Immunol. 7: 937-945.

19. Amadi-Obi, A., C. R. Yu, X. Liu, R. M. Mahdi, G. L. Clarke, R. B. Nussenblatt, I. Gery, Y. S. Lee, and C. E. Egwuagu. 2007. TH17 cells contribute to uveitis and scleritis and are expanded by IL-2 and inhibited by IL-27/STAT1. Nat. Med. 13: 711-718.

20. Kamiya, S., C. Nakamura, T. Fukawa, K. Ono, T. Ohwaki, T. Yoshimoto, and S. Wada. 2007. Effects of IL-23 and IL-27 on osteoblasts and osteoclasts: inhibitory effects on osteoclast differentiation. J. Bone Miner. Metab. 25: 277-285.

21. Wang, S., Y. Miyazaki, Y. Shinozaki, and H. Yoshida. 2007. Augmentation of antigen-presenting and Th1-promoting functions of dendritic cells by WSX-1(IL27R) deficiency. J. Immunol. 179: 6421-6428.

22. Lang, R. 2005. Tuning of macrophage responses by Stat3-inducing cytokines: molecular mechanisms and consequences in infection. Immunobiology 210 63-76.

23. Ivashkiv, L. B., and X. Hu. 2004. Signaling by STATs. Arthritis Res. Ther. 6: $159-168$.

24. Hu, X., C. Herrero, W. P. Li, T. T. Antoniv, E. Falck-Pedersen, A. E. Koch, J. M. Woods, G. K. Haines, and L. B. Ivashkiv. 2002. Sensitization of IFN- $\gamma$ Jak-STAT signaling during macrophage activation. Nat. Immunol. 3: 859-866.

25. Tassiulas, I., X. Hu, H. Ho, Y. Kashyap, P. Paik, Y. Hu, C. A. Lowell, and L. B. Ivashkiv. 2004. Amplification of IFN- $\alpha$-induced STAT1 activation and inflammatory function by Syk and ITAM-containing adaptors. Nat. Immunol. 5: 1181-1189.

26. Sharif, M. N., I. Tassiulas, Y. Hu, I. Mecklenbrauker, A. Tarakhovsky, and L. B. Ivashkiv. 2004. IFN- $\alpha$ priming results in a gain of proinflammatory function by IL-10: implications for systemic lupus erythematosus pathogenesis. J. Immunol. 172: 6476-6481

27. Ho, H. H., and L. B. Ivashkiv. 2006. Role of STAT3 in type I interferon responses: negative regulation of STAT1-dependent inflammatory gene activation. J. Biol. Chem. 281: 14111-14118

28. El Kasmi, K. C., J. Holst, M. Coffre, L. Mielke, A. de Pauw, N. Lhocine, A. M. Smith, R. Rutschman, D. Kaushal, Y. Shen, et al. 2006. General nature of the STAT3-activated anti-inflammatory response. J. Immunol. 177: 7880-7888.

29. Qing, Y., and G. R. Stark. 2004. Alternative activation of STAT1 and STAT3 in response to interferon- $\gamma$. J. Biol. Chem. 279: 41679-41685.

30. Costa-Pereira, A. P., S. Tininini, B. Strobl, T. Alonzi, J. F. Schlaak, H. Is'harc, I. Gesualdo, S. J. Newman, I. M. Kerr, and V. Poli. 2002. Mutational switch of an IL-6 response to an interferon- $\gamma$-like response. Proc. Natl. Acad. Sci. USA 99: 8043-8047.

31. Schroder, K., P. J. Hertzog, T. Ravasi, and D. A. Hume. 2004. Interferon- $\gamma$ : an overview of signals, mechanisms, and functions. J. Leukocyte Biol. 75: 163-189.

32. Moore, K. W., R. de Waal Malefyt, R. L. Coffman, and A. O'Garra. 2001. Interleukin-10 and the interleukin-10 receptor. Annu. Rev. Immunol. 19: 683-765.

33. Kamimura, D., K. Ishihara, and T. Hirano. 2003. IL-6 signal transduction and its physiological roles: the signal orchestration model. Rev. Physiol. Biochem. Pharmacol. 149: 1-38.

34. van Boxel-Dezaire, A. H., M. R. Rani, and G. R. Stark. 2006. Complex modulation of cell type-specific signaling in response to type I interferons. Immunity 25: $361-372$

35. Herrero, C., X. Hu, W. P. Li, S. Samuels, M. N. Sharif, S. Kotenko, and L. B. Ivashkiv. 2003. Reprogramming of IL-10 activity and signaling by IFN- $\gamma$. J. Immunol. 171: 5034-5041.

36. Artis, D., A. Villarino, M. Silverman, W. He, E. M. Thornton, S. Mu, S. Summer, T. M. Covey, E. Huang, H. Yoshida, et al. 2004. The IL-27 receptor (WSX-1) is an inhibitor of innate and adaptive elements of type 2 immunity. J. Immunol. 173: $5626-5634$

37. Murray, P. J. 2007. The JAK-STAT signaling pathway: input and output integration. J. Immunol. 178: 2623-2629.

38. Yoshimura, A., T. Naka, and M. Kubo. 2007. SOCS proteins, cytokine signalling, and immune regulation. Nat. Rev. Immunol. 7: 454-465.

39. Hu, X., P. K. Paik, J. Chen, A. Yarilina, L. Kockeritz, T. T. Lu, J. R. Woodgett, and L. B. Ivashkiv. 2006. IFN- $\gamma$ suppresses IL-10 production and synergizes with TLR2 by regulating GSK3 and CREB/AP-1 proteins. Immunity 24: 563-574.

40. Antoniv, T. T., K. H. Park-Min, and L. B. Ivashkiv. 2005. Kinetics of IL-10induced gene expression in human macrophages. Immunobiology 210: 87-95.

41. Saijo, S., N. Fujikado, T. Furuta, S. H. Chung, H. Kotaki, K. Seki, K. Sudo, S. Akira, Y. Adachi, N. Ohno, et al. 2007. Dectin-1 is required for host defense against Pneumocystis carinii but not against Candida albicans. Nat. Immunol. 8: 39-46.

42. Yoshida, H., Y. Miyazaki, S. Wang, and S. Hamano. 2007. Regulation of defense responses against protozoan infection by interleukin-27 and related cytokines. J. Biomed. Biotechnol. 2007: 79401.

43. Owaki, T., M. Asakawa, N. Morishima, K. Hata, F. Fukai, M. Matsui, J. Mizuguchi, and T. Yoshimoto. 2005. A role for IL-27 in early regulation of Th1 differentiation. J. Immunol. 175: 2191-2200.

44. Owaki, T., M. Asakawa, F. Fukai, J. Mizuguchi, and T. Yoshimoto. 2006. IL-27 induces Th1 differentiation via p38 MAPK/T-bet- and intercellular adhesion molecule-1/LFA-1/ERK1/2-dependent pathways. J. Immunol. 177: 7579-7587.

45. Sengupta, T. K., E. S. Talbot, P. A. Scherle, and L. B. Ivashkiv. 1998. Rapid inhibition of interleukin- 6 signaling and Stat 3 activation mediated by mitogenactivated protein kinases. Proc. Natl. Acad. Sci. USA 95: 11107-11112.

46. Ahmed, S. T., and L. B. Ivashkiv. 2000. Inhibition of IL-6 and IL-10 signaling and Stat activation by inflammatory and stress pathways. J. Immunol. 165: 5227-5237.

47. Niemand, C., A. Nimmesgern, S. Haan, P. Fischer, F. Schaper, R. Rossaint, P. C. Heinrich, and G. Muller-Newen. 2003. Activation of STAT3 by IL-6 and IL-10 in primary human macrophages is differentially modulated by suppressor of cytokine signaling 3. J. Immunol. 170: 3263-3272.

48. Fischer, P., U. Lehmann, R. M. Sobota, J. Schmitz, C. Niemand, S. Linnemann, S. Haan, I. Behrmann, A. Yoshimura, J. A. Johnston, et al. 2004. The role of the inhibitors of interleukin-6 signal transduction SHP2 and SOCS3 for desensitization of interleukin-6 signalling. Biochem. J. 378: 449-460.

49. Schaper, F., C. Gendo, M. Eck, J. Schmitz, C. Grimm, D. Anhuf, I. M. Kerr, and P. C. Heinrich. 1998. Activation of the protein tyrosine phosphatase SHP2 via the interleukin-6 signal transducing receptor protein gp130 requires tyrosine kinase Jak1 and limits acute-phase protein expression. Biochem. J. 335: 557-565.

50. Kim, H., T. S. Hawley, R. G. Hawley, and H. Baumann. 1998. Protein tyrosine phosphatase 2 (SHP-2) moderates signaling by gp130 but is not required for the induction of acute-phase plasma protein genes in hepatic cells. Mol. Cell. Biol. 18: $1525-1533$.

51. Kim, H., and H. Baumann. 1999. Dual signaling role of the protein tyrosine phosphatase SHP-2 in regulating expression of acute-phase plasma proteins by interleukin-6 cytokine receptors in hepatic cells. Mol. Cell. Biol. 19: 5326-5338.

52. VanDeusen, J. B , M. H Shah, B Becknell, B W Blaser, A K Ferketich, G. J. Nuovo, B. M. Ahmer, J. Durbin, and M. A. Caligiuri. 2006. STAT-1mediated repression of monocyte interleukin-10 gene expression in vivo. Eur. J. Immunol. 36: 623-630.

53. Zhong, Z., Z. Wen, and J. E. Darnell, Jr. 1994. Stat3: a STAT family member activated by tyrosine phosphorylation in response to epidermal growth factor and interleukin-6. Science 264: 95-98.

54. Schmitz, J., H. Dahmen, C. Grimm, C. Gendo, G. Muller-Newen, P. C. Heinrich, and F. Schaper. 2000. The cytoplasmic tyrosine motifs in full-length glycoprotein 130 have different roles in IL-6 signal transduction. J. Immunol. 164: 848-854.

55. Lehmann, U., J. Schmitz, M. Weissenbach, R. M. Sobota, M. Hortner, K. Friederichs, I. Behrmann, W. Tsiaris, A. Sasaki, J. Schneider-Mergener, et al. 2003. SHP2 and SOCS3 contribute to Tyr-759-dependent attenuation of interleukin-6 signaling through gp130. J. Biol. Chem. 278: 661-671.

56. Schmitz, J., M. Weissenbach, S. Haan, P. C. Heinrich, and F. Schaper. 2000. SOCS3 exerts its inhibitory function on interleukin-6 signal transduction through the SHP2 recruitment site of gp130. J. Biol. Chem. 275: 12848-12856.

57. Radtke, S., X. Yang, S. Wuller, B. Lippok, H. Schmitz-Van de Leur, B. Mutze, C. Stross, J. Bode, M. Gaestel, P. C. Heinrich, et al. 2007. Pro-inflammatory cytokines restrict IL-6 signalling through p38/MK2-mediated internalization and degradation of gp130. In Cytokines in Health and Disease: 15th Annual Meeting of the International Cytokine Society, October 26-30. International Cytokine Society, Atlanta, Abstract 223.

58. Villarino, A. V., J. Larkin, III, C. J. Saris, A. J. Caton, S. Lucas, T. Wong, F. J. de Sauvage, and C. A. Hunter. 2005. Positive and negative regulation of the IL-27 receptor during lymphoid cell activation. J. Immunol. 174: 7684-7691.

59. Ruckerl, D., M. Hessmann, T. Yoshimoto, S. Ehlers, and C. Holscher. 2006. Alternatively activated macrophages express the IL-27 receptor $\alpha$ chain WSX-1. Immunobiology 211: 427-436. 


\section{IL-27 inhibits human osteoclastogenesis by abrogating RANKL-mediated induction of NFATc1 and suppressing proximal RANK signaling}

Running title: IL-27 inhibits human osteoclastogenesis

George D. Kalliolias ${ }^{1 *}$ MD, Baohong Zhao* PhD, Antigoni Triantafyllopoulou* MD, KyungHyun Park-Min* PhD and Lionel B. Ivashkiv* ${ }^{\dagger}$ MD.

${ }^{1}$ Corresponding Author and reprint request

Address: Hospital for Special Surgery, 535 East $70^{\text {th }}$ Street, Research Building $4^{\text {th }}$ floor, New York, NY 10021 USA

Telephone number: 212-606-1651

Fax number: 212-774-2560

e-mail: kallioliasg@hss.edu

*Arthritis and Tissue Degeneration Program and Department of Medicine, Hospital for Special Surgery, New York, NY 10021.

${ }^{\dagger}$ Graduate Program in Immunology and Microbial Pathogenesis, Weill Cornell Graduate School of Medical Sciences New York, NY 10021.

This work was supported by grants from NIH (L.B.I), Niarchos Foundation (G.D.K) and Arthritis Foundation (K.H-P.M).

Keywords: Osteoclastogenesis, Cytokines, Interleukins, RANKL, Rheumatoid Arthritis 


\begin{abstract}
Objective. IL-27 has stimulatory and regulatory immune functions and is expressed in rheumatoid arthritis synovium. We investigated the effects of IL-27 on human osteoclastogenesis to determine whether IL-27 can stimulate or attenuate osteoclast-mediated bone resorption that is a hallmark of rheumatoid arthritis.

Methods. Osteoclasts were generated from blood-derived human CD14+ cells. The effects of IL-27 on osteoclast formation were evaluated by counting the number of TRAP+ multinucleated cells and measuring expression of osteoclast-related genes. The induction of NFATc1 and the activation of signaling pathways downstream of RANK were measured by immunoblotting. The expression of key molecules implicated in osteoclastogenesis (NFATc1, RANK, costimulatory receptors, ITAM-harboring adaptors) was measured by real time RT-PCR. Murine osteoclast precursors were obtained from bone marrow. Responsiveness to IL-27 of synovial fluid macrophages derived from RA patients was also tested.
\end{abstract}

Results. IL-27 inhibited human osteoclastogenesis, suppressed the induction of NFATc1, downregulated expression of RANK and TREM-2, and inhibited RANKL-mediated activation of

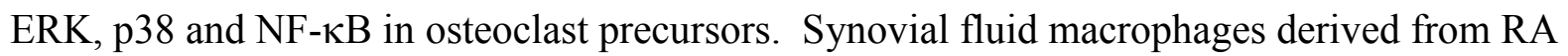
patients were refractory to the effects of IL-27. In contrast to humans, IL-27 only moderately suppressed murine osteoclastogenesis, likely due to low expression of the IL-27 receptor subunit WSX-1 on murine osteoclast precursors.

Conclusion. IL-27 inhibits human osteoclastogenesis by a direct mechanism suppressing responses of osteoclast precursors to RANKL. Our findings suggest that in addition to its wellknown anti-inflammatory effects, IL-27 plays a homeostatic role in restraining bone erosion. 
This homeostatic function is compromised under conditions of chronic inflammation such as RA synovitis. 
IL-27 is a member of the IL-12 family of the heterodimeric cytokines that also includes IL-12, IL-23 and IL-35 (1-3). It is comprised of EBI3 (EBV-induced protein 3) and p28 subunits that share similarity with, respectively, the p40 and p35 subunits of IL-12 (4). The IL-27 receptor is a heterodimer composed of a WSX-1 subunit (also termed TCCR, T cell cytokine receptor), which confers ligand specificity, and the gp130 signaling subunit that is also utilized by the IL-6 family of cytokines (5). IL-27 activates the Jak-STAT signal transduction pathway in a context dependent manner, depending on cell type and activation state. In resting lymphocytes, IL-27 activates STAT1, STAT3, STAT5 and low amounts of STAT4 (6), whereas activation of STAT1 is decreased in fully activated $\mathrm{CD}^{+} \mathrm{T}$ cells relative to resting cells (7). In myeloid cells IL-27 induces phosphorylation of STAT1 and STAT3 $(5,8,9)$ and we have recently reported that in human monocytes IL-27 has a STAT1-dominant effect (10). In contrast, we have found that murine BMDM are minimally responsive to IL-27.

IL-27 plays both activating and regulatory roles in immune responses (2). IL-27 is produced early during innate responses and augments the induction of Th1 responses $(4,6)$. In contrast, later in the evolution of an immune response IL-27 suppresses Th1 polarization and inhibits Th17 and Th2 differentiation (6, 11-14), in part by inducing IL-10 production (15-17). The role of IL-27 in arthritis and other inflammatory or infectious diseases has been explained on the basis of its effects on T cell differentiation (1-3). IL-27 can play either a pathogenic or a protective role in murine models of inflammatory arthritis, depending upon the model and underlying pathogenic mechanisms. In adjuvant-induced arthritis and proteoglycan-induced arthritis, both considered Th1-mediated diseases, IL-27 is pathogenic, consistent with its known Th1-inducing effects $(18,19)$. In contrast, in collagen-induced arthritis, which is Th17-mediated (and where Th1 responses can actually be protective), IL-27 was protective and proposed as a 
potential treatment for arthritis (20). IL-27 is expressed in human rheumatoid arthritis (20), which has been considered a Th1-mediated disease, but recent evidence suggests a role for Th17 responses in disease pathogenesis (21). Thus, it is important to determine whether IL-27 plays a pathogenic or protective role in human RA.

Rheumatoid arthritis is characterized by chronic synovial inflammation and bone destruction and a hallmark of disease is bone erosions mediated by osteoclasts (22). In the last few years, significant breakthroughs have improved our understanding of mechanisms that control the activity of osteoclasts under physiologic and pathologic circumstances $(23,24)$. Osteoclasts are large multinucleated cells created by the differentiation and fusion of myeloid lineage precursor cells, which include cells in the blood monocyte pool (25). In the microenvironment of bone, myeloid-derived osteoclast precursors are exposed to stimuli that commit them to the osteoclast lineage (26). The driving force of this commitment-differentiation process is the interaction of RANKL (a cytokine member of the TNF super-family) with its receptor RANK that is expressed on the surface of osteoclast precursors $(27,28)$. Effective osteoclastogenesis requires co-stimulatory calcium-mediated signals by ITAM-coupled receptors that co-operate with RANK signaling to induce NFATc1 and the downstream osteoclast differentiation program (29-32). In humans, TREM-2 is a key costimulatory receptor, as loss of function mutations in TREM-2 compromise bone remodeling and lead to Nasu Hakola disease $(33,34)$.

The multi-step osteoclast differentiation program is tightly controlled by hormones, cytokines and other regulatory factors. Cytokines that activate the Jak-STAT signal transduction pathway have been implicated in the regulation of osteoclastogenesis bridging immune regulation with bone remodeling. IFNs $(35,36)$, IL-4 (37), IL-6 (38) and IL-10 (39) inhibit 
osteoclast differentiation, while IL-23 promotes osteoclast formation by up-regulating RANK expression in precursor cells (40). IL-27, which belongs to the same cytokine family as IL-23, has been reported to modestly and indirectly regulate osteoclast differentiation in murine systems $(40,41)$, but mechanisms by which IL-27 regulates osteoclastogenesis and the role of IL-27 in human osteoclastogenesis have not been addressed.

We wished to explore the role of IL-27 in human osteoclastogenesis as this could yield insight into one facet of the potential pathogenic vs. protective roles of IL-27 in human RA. We found that IL-27 is a potent inhibitor of human osteoclastogenesis by a direct effect on osteoclast precursor cells. One mechanism that mediates the anti-osteoclastogenic function of IL-27 is abrogation of RANKL-induced c-Jun and NFATc1 expression by downregulation of the expression of RANK and inhibition of MAPK- and NF-kB signaling pathways downstream of RANK. We also found that in osteoclast precursor cells IL-27 downregulates the expression of the TREM-2 costimulatory receptor. Surprisingly, we found that synovial fluid derived macrophages from joint effusions of patients with active RA were refractory to the effects of IL27. Our findings, combined with the reported inhibitory effects of IL-27 on Th17-mediated inflammation, suggest that IL-27 has the capacity to regulate both chronic inflammation and associated bone erosion, but this regulatory function is attenuated within the inflammatory microenvironment of the joint. 


\section{Materials and Methods}

Cell culture. PBMCs from healthy volunteers' blood, purchased from the NY Blood Center, were obtained by density gradient centrifugation. Mononuclear cells were isolated from synovial fluids derived from five patients with rheumatoid arthritis (diagnosis was based on ACR criteria). $\mathrm{CD}_{1} 4^{+}$cells were purified from PBMCs and from synovial fluid derived mononuclear cells using anti-CD14 magnetic beads (Miltenyi Biotec). Murine monocytes were obtained from C57BL/6J mice (The Jackson Laboratory) by density gradient centrifugation and positive selection using anti-CD11b magnetic beads (Miltenyi Biotec). Bone marrow cells and splenocytes were obtained from C57BL/6J mice and bone marrow-derived osteoclast precursors were generated by 4 days of culture on Petri dishes in DMEM supplemented with 20\% FBS (HyClone) and recombinant murine M-CSF (20 ng/ml) (Peprotech). To measure cell viability, MTT assays were performed using an MTT assay kit (Roche Diagnostics, Indianapolis, IN). Experiments with human cells were approved by the Hospital for Special Surgery IRB and with mouse cells by the Institutional Animal Care and Use Committee.

Osteoclast differentiation. Human $\mathrm{CD} 14^{+}$cells were incubated in $\alpha$-MEM (Invitrogen) supplemented with 10\% FBS (Defined, Hyclone) and 20ng/ml of hM-CSF (PeproTech) for 2 days to generate osteoclast precursors. Osteoclast precursors were incubated with $20 \mathrm{ng} / \mathrm{ml}$ of MCSF and 40ng/ml of sRANKL (PeproTech) for an additional 5 days. Cytokines were replenished every 3 days. On culture day 7, cells were fixed and stained for TRAP using the Acid Phosphatase Leukocyte diagnostic kit (Sigma, San Diego, CA). Multinucleated (> 3 nuclei) TRAP-positive osteoclasts were counted in triplicate wells of 96 well plates. Recombinant hIL-27 (R\&D Systems) was added either from the beginning of the culture and 
before RANKL (IL-27 pretreatment), simultaneously with RANKL on culture day 3, or after RANKL (on culture day 5). Murine bone marrow-derived osteoclast precursors were incubated with mMCSF $(20 \mathrm{ng} / \mathrm{ml})$ and sRANKL $(80 \mathrm{ng} / \mathrm{ml})$ for 5 days in $\alpha$-MEM above.

Pit formation assay. Human preosteoclasts were re-plated on dentin slices $\left(5 \times 10^{4}\right.$ cells/slice $)$ in 96-well culture plates and stimulated as indicated. Cells on dentin were removed, and the dentin slices were immersed in $1 \%$ toluidine blue $\mathrm{O}$ (Sigma-Aldrich) to stain resorption pits formed by mature osteoclasts.

Immunoblotting. Whole-cell extracts were prepared by lysis of cells in buffer containing 20 $\mathrm{mM}$ Hepes (pH 7.0), $300 \mathrm{mM} \mathrm{NaCl}, 10 \mathrm{mM} \mathrm{KCl}, 1 \mathrm{mM} \mathrm{MgCl} 2,0.1 \%$ Triton X-100, $0.5 \mathrm{mM}$ DTT, 20\% glycerol and $1 \times$ proteinase inhibitor cocktail (Roche, Basel, Switzerland). Nuclear extracts were prepared by incubating cells for $15 \mathrm{~min}\left(4^{\circ} \mathrm{C}\right)$ in buffer containing $10 \mathrm{mM}$ Hepes (pH 7.9), $1.5 \mathrm{mM} \mathrm{MgCl}_{2}, 10 \mathrm{mM} \mathrm{KCl}, 1 \times$ proteinase inhibitor cocktail and $1 \mathrm{mM}$ DTT. NP-40 was added to a final concentration of $0.2 \%$ and the lysate was centrifuged at $10,000 \mathrm{~g}$ for 30sec. Finally, the nuclear pellet was lysed in SDS-PAGE loading buffer.

For immunoblotting 5 or $10 \mu \mathrm{g}$ of whole-cell lysates were fractioned on polyacrylamide gels using SDS-PAGE, transferred to polyvinylidene fluoride membranes (Millipore) and incubated with specific Abs. ECL was used for detection. ERK, p-ERK, p-p38, p-IкB $\alpha, \operatorname{I\kappa B} \alpha, \mathrm{c}-J u n, p Y-$ STAT1 and pY-STAT3 antibodies were from Cell Signaling Technology. TRAF6, TBP and p38 antibodies were from Santa Cruz Biotechnology while NFATc1, STAT1 and STAT3 antibodies were purchased from BD Transduction Laboratories. 
RT-PCR and quantitative RT-PCR (qPCR). For RT-PCR and qPCR total RNA was extracted using an RNeasy mini kit (Qiagen) and $1 \mu \mathrm{g}$ of total RNA was reverse transcribed using a First Strand cDNA Synthesis kit (Fermentas). qPCR was performed using iQ SYBR Green Supermix and iCycler iQ thermal cycler (Bio-Rad). PCR $\left(34\right.$ cycles: $95^{\circ} \mathrm{C} 30 \mathrm{sec} / 60^{\circ} \mathrm{C} 30 \mathrm{sec} / 72^{\circ} \mathrm{C} 1 \mathrm{~min}$.) was utilized to quantify the mRNA levels of WSX-1 in human CD14+ or CD14- cells and murine osteoclast precursors or splenocytes. 


\section{Results}

\section{IL-27 inhibits human osteoclastogenesis in a dose- and time-dependent manner.}

In the presence of M-CSF and RANKL myeloid-lineage precursors differentiate into osteoclasts (25), but STAT1 activation downstream of IFNs has been shown to inhibit osteoclastogenesis (42). Recently we reported that IL-27 strongly activates STAT1 in human monocytes and macrophages (10) and in this study we wished to investigate whether IL-27 has any regulatory function on human osteoclastogenesis. As expected (43), culture with M-CSF plus RANKL induced differentiation of large multinucleated TRAP+ cells that were readily apparent 5 days after RANKL addition (Figure 1A, second panel and Figure 1B). When IL-27 was added at the beginning of culture (Day 1), a dose dependent inhibition of osteoclastogenesis was observed (Figure 1A and 1B), with nearly complete inhibition of generation of multinucleated TRAP+ cells observed at doses $\geq 30 \mathrm{ng} / \mathrm{ml}$ of IL-27 ( $\mathrm{p}<0.01$, Student's t test). When IL-27 (100ng/ml) was added at later time points during the differentiation process, a substantial inhibition was observed when IL-27 was added on day 3 (simultaneously with addition of RANKL), while there was slight inhibition when IL-27 was added on day 5 (Figure 1A and 1B). A similar pattern of dose- and time-dependent inhibition of RANKL-induced expression of osteoclast-related genes Cathepsin K (Figure 1C) and integrin $\beta 3$ (data not shown) by IL-27 was observed. Cathepsin K and integrin $\beta 3$ were still expressed in the mostly mononuclear cells observed when $30 \mathrm{ng} / \mathrm{ml}$ of IL-27 was used, but were strongly downregulatd by saturating concentrations of IL-27 that nearly completely suppressed osteoclastogenesis. The functional consequence of the observed IL-27-mediated inhibition of osteoclastogenesis was assessed by resorption pit formation assay using dentin slices. As expected, the observed inhibition of osteoclastogenesis by IL-27 was reflected also by the absence of resorption pits (Figure 1D, left). Interestingly, when IL-27 was 
added late on day 5 , the formed osteoclasts were capable of creating resorption pits (Figure 1D, left, fifth panel). The latter implies that IL-27 can not inhibit the late stages of osteoclast differentiation and function. Additionally, we found using the MTT assay that IL-27 mediates antiosteoclastogenic effects without affecting viability of osteoclast precursors (Figure 1D, right). Taken together, these observations suggest that IL-27 is a potent inhibitor of the early stages of human osteoclastogenesis by a direct effect on osteoclast precursors.

\section{IL-27 abrogates RANKL-mediated induction of NFATc1.}

We wished to investigate mechanisms by which IL-27 inhibits osteoclast differentiation. It is well established that NFATc1 is a master regulator of osteoclastogenesis, driving the expression of genes that are crucial for the commitment and differentiation of precursor cells to osteoclasts $(44,45)$. We tested the effects of IL-27 on NFATc1 expression. As expected, RANKL (40ng/ml) induced within 24h NFATc1 protein expression (Figure 2A, lane 4) and the levels of NFATc1 protein remained elevated for at least 72h following RANKL stimulation (Figure 2A, lanes 4-6). Addition of IL-27 resulted in the abrogation of RANKL-induced NFATc1 protein expression for the entire 72h period of RANKL stimulation (Figure 2A, lanes 7-9). We next investigated whether IL-27 inhibits NFATc1 gene expression. The levels of NFATc1 mRNA increased following 24 and 48h stimulation with RANKL (Figure 2B). Pretreatment with IL-27

resulted in substantial suppression of NFATc1 mRNA levels (Figure 2B). A similar striking abrogation of RANKL-induced NFATc1 protein expression was observed when IL-27 was added together with RANKL (on culture day 3) (Figure 2C, lanes 4-9). Simultaneous addition of IL-27 had a similar suppressive effect on NFATc1 mRNA expression (Figure 2D). These results 
indicate that the abrogation of NFATc1 induction is a mechanism that mediates the inhibitory effects of IL-27 on human osteoclastogenesis.

\section{IL-27 inhibits RANKL-induced activation of MAPK and NF-KB pathways and suppresses RANK expression.}

The induction of NFATc1 expression by RANK is dependent upon RANKL-induced activation of MAPK and NF-kB pathways $(23,24)$, and thus we investigated whether IL-27 inhibited RANK signaling. In agreement with the literature, we found that stimulation of osteoclast precursors with RANKL (40ng/ml) induced rapid phosphorylation of ERK and p38 MAPKs (Figure 3A, first and third panels, lanes 2-4). We observed strong inhibition of RANKL-induced ERK and p38 phosphorylation by IL-27 (Figure 3A, first and third panels, lanes 6-8). In addition, RANKL induced the expected phosphorylation and rapid degradation of IKB $\alpha$ (Figure 3B, top panels, lanes 2-4) indicating activation of the classical NF- $\mathrm{kB}$ pathway, while IL-27 prevented RANKL-induced IкB $\alpha$ phosphorylation and degradation (Figure 3B, top panels, lanes 6-8). IFN $\gamma$, the prototypic STAT1 activating cytokine, inhibits RANKL signaling in murine osteoclast precursors by inducing rapid degradation of the adapter TRAF6 that lies upstream of NF- $\mathrm{KB}$ and MAPK activation (35). In our system IL-27 had no effect on the expression of TRAF6 protein (Figure 3B, bottom panel). RANKL-mediated induction of AP-1 proteins, including Fos and Jun, is important for osteoclastogenesis. Induction of Fos by RANKL was not consistently observed in our human osteoclastogenesis system (data not shown). However, RANKL consistently induced c-Jun protein and this induction was detectable in nuclear extracts for at least $3 \mathrm{~h}$ following RANKL stimulation (Figure 3C, lanes 2-4). In the presence of IL-27 the RANKL-mediated induction of c-Jun was abrogated (Figure 3C, lanes 6-8). We then 
investigated whether IL-27 inhibits expression of RANK, which could explain IL-27-mediated inhibition of several RANK signaling pathways. Culture with MCSF induced the expected increase in RANK mRNA that peaked at $24 \mathrm{~h}$ and remained elevated at $48 \mathrm{~h}$ of culture (Figure 3D, black bars). IL-27 partially suppressed M-CSF-mediated induction of RANK mRNA expression in all donors tested $(>10)$ (Figure 3D, grey bars). When we tested the effects of IL-27 on the expression of the M-CSF receptor c-Fms, there was variability among donors in the effects of IL-27 on c-Fms mRNA levels and cell surface expression, while IL-27 strongly inhibited osteoclastogenesis in all donors tested $(n>10)$. Because IL-27 inhibited osteoclastogenesis effectively even in donors where c-Fms expression was not affected, we conclude that an effect of IL-27 on c-Fms expression is not a major mechanism to explain the observed inhibition of osteoclastogenesis. In sum, the results demonstrate that IL-27 inhibits RANK-induced signaling that is required for NFATc1 expression, and suggest that this inhibition may be mediated in part by downregulation of RANK expression.

\section{IL-27 suppresses TREM-2 mRNA expression}

As the strong inhibition of osteoclastogenesis and NFATc1 expression by IL-27 could not be solely explained by inhibition of RANK expression, we investigated the effects of IL-27 on other pathways implicated in the induction of NFATc1 expression. The immunonoreceptors TREM-2, OSCAR, SIRP $\beta 1$ and ILT7 (homolog of murine PIR-A) and integrin $\alpha$ V $\beta 3$ that signal via ITAM-containing adaptors DAP12 and FcR $\gamma$ cooperate with RANK signaling pathways to induce NFATc1 expression and osteoclastogenesis (29-32). We investigated the effects of IL-27 on costimulatory receptors and DAP12/FcR $\gamma$ expression. The expression of TREM-2 increased during the early differentiation of osteoclast precursors, and this increase in TREM-2 expression 
was strongly suppressed by IL-27 (Figure 4A). In contrast, expression of OSCAR, SIRP $\beta 1$, DAP12 and FcR $\gamma$, did not substantially change during differentiation of osteoclast precursors, and was not suppressed by IL-27 (Figure 4B and 4C). ILT7 mRNA expression was moderately increased by IL-27 (Figure 4B). As costimulatory signaling is important for NFATc1 expression and TREM-2 plays a key role in costimulation of human osteoclastogenesis $(33,34)$, these observations suggest down-regulation of TREM-2 as a mechanism by which IL-27 suppresses NFATc1 induction and inhibits human osteoclastogenesis.

\section{Synovial fluid macrophages derived from RA patients are refractory to IL-27.}

Given the above described inhibitory effects of IL-27 in human osteoclastogenesis, we wondered whether IL-27 exerts these regulatory functions in $\mathrm{CD} 14^{+}$cells derived from synovial fluid (SF) of patients with active rheumatoid arthritis. SF-derived $\mathrm{CD} 14^{+}$cells from RA patients responded to IFN- $\gamma$ stimulation with activation of STAT1 (Figure $5 \mathrm{~A}$, first panel, lane 2) and a robust induction of CXCL10 gene expression (Figure 5B, white bar). Surprisingly, following stimulation with IL-27 we observed a very faint activation of STAT1 (Figure 5A, top panel, lane 3) that was inadequate to induce STAT1-target gene expression (including CXCL10, CXCL9, IRF-1 and STAT1, Figure 5B and data not shown; $p<0.001$, Student's $t$ test). These results were consistently observed in synovial macrophages from all five patients tested and suggest that cells derived from the joints of many patients with active RA are only minimally responsive to IL-27. The latter implies that, within the microenvironment of an inflamed joint, IL-27 may not exert its regulatory functions. 


\section{IL-27 moderately inhibits murine osteoclastogenesis due to low expression of WSX-1.}

We have recently reported that murine macrophages are minimally responsive to IL-27 (10) and in this study we wished to test whether IL-27 has an effect on murine osteoclast differentiation. Because we wished to use murine monocytes as osteoclast precursors (to directly compare to human monocytes), we first tested the responsiveness of murine monocytes to IL-27. Following stimulation of murine monocytes with IFN- $\gamma$ (that was used as a positive control) we observed the expected activation of STAT1 and STAT3 (Figure 6A, first and third panel, lanes 2-4) and induction of classical STAT1-target genes (including IP-10, MIG, IRF-1, data not shown). Similarly to murine macrophages (10), in murine monocytes following IL-27 stimulation there was no detectable activation of STAT1 and STAT3 (Figure 6A, first and third panel, lanes 5-7) and minimal induction of STAT1-target genes (data not shown). Because murine monocytes did not differentiate efficiently into osteoclasts in our system, we used bone marrow-derived osteoclast precursors, a standard approach to study murine osteoclastogenesis, to test the effects of IL-27. As expected, addition of RANKL to murine bone marrow-derived osteoclast precursors resulted in the formation of large multinucleated TRAP+ cells within 5 days (Figure 6B, upper

panels). When IL-27 was added (prior to or simultaneously with RANKL) in doses ranging from $1-100 \mathrm{ng} / \mathrm{ml}$ there was a minimal to moderate inhibition of osteoclast formation (Figure 6B, panels 3-5). Similarly, the number of TRAP+ multinucleated cells (Figure 6C) and expression of osteoclast marker genes (data not shown) were only moderately lower in the presence of IL-27. In stark contrast to the human system, IL-27 had minimal effect on RANKL-mediated induction of NFATc1 expression in murine osteoclast precursors (data not shown), further supporting the notion that murine osteoclast precursors are moderately responsive to IL-27. In our recent report (10), we have shown that IL-6 induces a substantial activation of STAT-3 in murine myeloid 
cells, indicating that gp130, the shared receptor subunit for IL-6 and IL-27 receptors, is

expressed on these cells. We next investigated the expression levels of the WSX-1 subunit of the IL-27 receptor in human and murine cells. We found that human lymphocytes and osteoclast precursors express WSX-1 mRNA at comparable levels (Figure 6D, lanes 1-2), while WSX-1 expression is low in murine osteoclast precursors compared to murine splenocytes

(predominantly lymphocytes) (Figure 6D, lanes 3-4). The latter suggests that low expression of WSX-1 in murine osteoclast precursors is a potential explanation for the modest responsiveness of these cells to the effects of IL-27.

\section{Discussion}

In this study we have found that IL-27 is a potent inhibitor of human osteoclastogenesis. This effect is mediated by directly targeting the early stages of differentiation of precursor cells into osteoclasts. In the presence of IL-27 we observed down-regulation of RANK and TREM-2 expression, an inhibition of MAPK- and NF-kB- pathways downstream of RANK and most importantly an abrogation of RANKL-induced c-Jun and NFATc1 expression. In stark contrast to blood-derived $\mathrm{CD} 14^{+}$cells from healthy donors, SF-derived $\mathrm{CD} 14^{+}$cells from RA patients were refractory to IL-27. Our observations, combined with the well-characterized antiinflammatory functions of IL-27 (3), suggest that IL-27 has the potential to limit the extent of bone destruction in the setting of infection or inflammation, but that this homeostatic function of IL-27 can be compromised during chronic inflammation, such as occurs in rheumatoid arthritis.

NFATc1 is essential for osteoclast differentiation in vitro and in vivo $(44,45)$. We observed a remarkable suppression of NFATc1 mRNA and protein expression by IL-27 in our 
system. Signaling pathways downstream of RANK and immunoreceptors (including TREM-2) co-operate leading to the activation of calcium signaling, the activation of NFATc1 and finally strong induction of NFATc1 itself through an auto-amplification loop (24). In our study, the

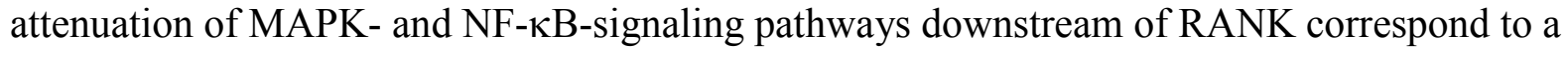
mechanism that contributes to the suppression of NFATc1 expression by IL-27. IL-27 appears to regulate the levels of c-Jun protein that is induced downstream of MAPK pathways. c-Jun is the partner of c-Fos for the formation AP-1, a transcription factor essential for the induction of NFATc1 (46). A potential explanation for the inhibition of the MAPK and NF- $\kappa$ B signaling pathways observed in our system is the down-regulation of RANK expression by IL-27 in human osteoclast progenitors. This downregulation of RANK was partial, suggesting that it contributes to diminished responses to RANKL only under conditions where osteoclast precursors are exposed to low concentrations of RANKL (as in the case of the inflamed synovium in rheumatoid arthritis). In contrast, TREM-2 expression was effectively inhibited by IL-27, indicating that IL-27 suppresses costimulation of RANK signaling.

We have previously reported that IL-27 has a STAT1-dominant, IFN $\gamma$-like effect on human monocytes (10). IFN $\gamma$ is also a powerful suppressor of osteoclast differentiation by a STAT1-mediated mechanism (42). Similar to our observations with IL-27, IFN- $\gamma$ inhibits the activation of MAPK and NF- $\kappa$ B pathways downstream of RANK (35). IFN $\gamma$ inhibits RANK signaling in murine osteoclast precursors by inducing rapid degradation of the signaling adapter TRAF6 that functions downstream of RANK (35). In our system the protein levels of TRAF6 were not reduced in IL-27 treated cells prior to or after RANKL stimulation, indicating that there was no overt degradation of TRAF6. This observation suggests that IL-27 and IFN $\gamma$ inhibit osteoclastogenesis by different mechanisms, although it is possible that differences in TRAF6 
protein regulation and stability between murine and human osteoclast precursors contributes to the observed differences.

In the current study we have found that SF macrophages derived from joint effusions of patients with active RA retain responsiveness to IFN- $\gamma$ but are refractory to IL-27. We have recently reported that resistance to IL-27 can be induced by LPS by a p38-dependent mechanism (10). These findings suggest that inflammatory factors that activate p38 can induce a state of refractoriness to IL-27, and in this context we hypothesize that, within the inflammatory microenvironment of the joint, where $\mathrm{p} 38$ is known to be activated, SF macrophages are exposed to stimuli that render them refractory to the regulatory effects of IL-27. The level of refractoriness is likely to wax and wane with disease activity, but the data suggest that patients with active disease would be refractory to IL-27 therapy. However, greater understanding of mechanisms that render cells refractory to IL-27 could potentially lead to therapeutic manipulations to restore cell responsiveness to the regulatory functions of IL-27. Under such conditions, endogenous IL-27 would have a beneficial effect in suppressing bone resorption, and exogenous IL-27 might represent an effective therapy.

Recently, several differences have become apparent in the regulation of osteoclastogenesis and bone remodeling between humans and mice (47). For example, TREM-2 deficiency in humans is associated with impaired osteoclastogenesis, aberrant bone remodeling and Nasu-Hakola disease (also called polycystic lipomembranous osteodysplasia with sclerosing leukoengephalopathy) $(33,34)$. In contrast, mice deficient in TREM-2 do not have a clear bone phenotype in vivo and have increased osteoclastogenesis in vitro (48). Our observations add to these species differences by showing substantially less effective inhibition of osteoclast differentiation by IL-27 with mouse relative to human osteoclast precursors. One potential 
explanation of this difference is that murine osteoclast precursors are moderately responsive to IL-27 due to low expression of WSX-1. An additional explanation is likely related to differences in TREM-2 regulation and function in humans and mice, and the contribution of TREM-2 downregulation to the suppressive effects of IL-27 in human cells. In addition, we have shown that in human cells IL-27 is a powerful inhibitor of osteoclastogenesis directly targeting osteoclast progenitors, while our findings with murine bone marrow-derived osteoclast progenitors and evidence from other groups suggest that in murine systems IL-27 regulates osteoclast differentiation mainly indirectly by affecting cell populations other than the osteoclast precursors. It has been reported that IL-27 induces activation of STAT-1 and STAT-3 in murine osteoblasts, but no profound functional consequences were found (41). In the same study, IL-27 inhibited osteoclastogenesis indirectly possibly by regulating the functions of activated CD4+ Tcells. Along the same lines, another study identified murine Th17 cells as the exclusive osteoclastogenic subset among the known CD4+ T-cell lineages (49) and several reports suggest that IL-27 suppresses Th17 polarization $(12,13,50)$.

The findings of our current study provide evidence that IL-27 is a powerful inhibitor of human osteoclastogenesis, but the perspective of using IL-27 as a potential treatment in human diseases including rheumatoid arthritis needs further investigation and careful evaluation. 


\section{References}

1. Kastelein RA, Hunter CA, Cua DJ. Discovery and biology of IL-23 and IL-27: related but functionally distinct regulators of inflammation. Annu Rev Immunol 2007;25:221-42.

2. Yoshida H, Yoshiyuki M. Regulation of immune responses by interleukin-27. Immunol Rev 2008;226:234-47.

3. Batten M, Ghilardi N. The biology and therapeutic potential of interleukin 27. J Mol Med 2007;85(7):661-72.

4. Pflanz S, Timans JC, Cheung J, Rosales R, Kanzler H, Gilbert J, et al. IL-27, a heterodimeric cytokine composed of EBI3 and p28 protein, induces proliferation of naive CD4(+) T cells. Immunity 2002;16(6):779-90.

5. Pflanz S, Hibbert L, Mattson J, Rosales R, Vaisberg E, Bazan JF, et al. WSX-1 and glycoprotein 130 constitute a signal-transducing receptor for IL-27. J Immunol 2004;172(4):2225-31.

6. Lucas S, Ghilardi N, Li J, de Sauvage FJ. IL-27 regulates IL-12 responsiveness of naive CD4+ T cells through Stat1-dependent and -independent mechanisms. Proc Natl Acad Sci U S A 2003;100(25):15047-52.

7. Yoshimura T, Takeda A, Hamano S, Miyazaki Y, Kinjyo I, Ishibashi T, et al. Two-sided roles of IL-27: induction of Th1 differentiation on naive CD4+ T cells versus suppression of proinflammatory cytokine production including IL-23-induced IL-17 on activated CD4+ T cells partially through STAT3-dependent mechanism. J Immunol 2006;177(8):5377-85.

8. Lang R. Tuning of macrophage responses by Stat3-inducing cytokines: molecular mechanisms and consequences in infection. Immunobiology 2005;210(2-4):63-76.

9. Wang S, Miyazaki Y, Shinozaki Y, Yoshida H. Augmentation of antigen-presenting and Th1-promoting functions of dendritic cells by WSX-1(IL-27R) deficiency. J Immunol 2007;179(10):6421-8.

10. Kalliolias GD, Ivashkiv LB. IL-27 activates human monocytes via STAT1 and suppresses IL-10 production but the inflammatory functions of IL-27 are abrogated by TLRs and p38. J Immunol 2008;180(9):6325-33.

11. Villarino A, Hibbert L, Lieberman L, Wilson E, Mak T, Yoshida H, et al. The IL-27R (WSX-1) is required to suppress T cell hyperactivity during infection. Immunity 2003;19(5):64555.

12. Batten M, Li J, Yi S, Kljavin NM, Danilenko DM, Lucas S, et al. Interleukin 27 limits autoimmune encephalomyelitis by suppressing the development of interleukin 17-producing $\mathrm{T}$ cells. Nat Immunol 2006;7(9):929-36.

13. Amadi-Obi A, Yu CR, Liu X, Mahdi RM, Clarke GL, Nussenblatt RB, et al. TH17 cells contribute to uveitis and scleritis and are expanded by IL-2 and inhibited by IL-27/STAT1. Nat Med 2007;13(6):711-8.

14. Artis D, Villarino A, Silverman M, He W, Thornton EM, Mu S, et al. The IL-27 receptor (WSX-1) is an inhibitor of innate and adaptive elements of type 2 immunity. J Immunol 2004;173(9):5626-34.

15. Fitzgerald DC, Zhang GX, El-Behi M, Fonseca-Kelly Z, Li H, Yu S, et al. Suppression of autoimmune inflammation of the central nervous system by interleukin 10 secreted by interleukin 27-stimulated T cells. Nat Immunol 2007;8(12):1372-9. 
16. Awasthi A, Carrier Y, Peron JP, Bettelli E, Kamanaka M, Flavell RA, et al. A dominant function for interleukin 27 in generating interleukin 10-producing anti-inflammatory T cells. Nat Immunol 2007;8(12):1380-9.

17. Stumhofer JS, Silver JS, Laurence A, Porrett PM, Harris TH, Turka LA, et al.

Interleukins 27 and 6 induce STAT3-mediated T cell production of interleukin 10. Nat Immunol 2007;8(12):1363-71.

18. Goldberg R, Wildbaum G, Zohar Y, Maor G, Karin N. Suppression of ongoing adjuvantinduced arthritis by neutralizing the function of the p28 subunit of IL-27. J Immunol 2004;173(2):1171-8.

19. Cao Y, Doodes PD, Glant TT, Finnegan A. IL-27 induces a Th1 immune response and susceptibility to experimental arthritis. J Immunol 2008;180(2):922-30.

20. Niedbala W, Cai B, Wei X, Patakas A, Leung BP, McInnes IB, et al. Interleukin 27 attenuates collagen-induced arthritis. Ann Rheum Dis 2008;67(10):1474-9.

21. Tesmer LA, Lundy SK, Sarkar S, Fox DA. Th17 cells in human disease. Immunol Rev 2008;223:87-113.

22. Firestein GS. Evolving concepts of rheumatoid arthritis. Nature 2003;423(6937):356-61.

23. Tanaka S, Nakamura K, Takahasi N, Suda T. Role of RANKL in physiological and pathological bone resorption and therapeutics targeting the RANKL-RANK signaling system. Immunol Rev 2005;208:30-49.

24. Takayanagi H. Osteoimmunology: shared mechanisms and crosstalk between the immune and bone systems. Nat Rev Immunol 2007;7(4):292-304.

25. Xing L, Schwarz EM, Boyce BF. Osteoclast precursors, RANKL/RANK, and immunology. Immunol Rev 2005;208:19-29.

26. Boyle WJ, Simonet WS, Lacey DL. Osteoclast differentiation and activation. Nature 2003;423(6937):337-42.

27. Lacey DL, Timms E, Tan HL, Kelley MJ, Dunstan CR, Burgess T, et al. Osteoprotegerin ligand is a cytokine that regulates osteoclast differentiation and activation. Cell 1998;93(2):16576.

28. Yasuda H, Shima N, Nakagawa N, Yamaguchi K, Kinosaki M, Mochizuki S, et al. Osteoclast differentiation factor is a ligand for osteoprotegerin/osteoclastogenesis-inhibitory factor and is identical to TRANCE/RANKL. Proc Natl Acad Sci U S A 1998;95(7):3597-602. 29. Humphrey MB, Lanier LL, Nakamura MC. Role of ITAM-containing adapter proteins and their receptors in the immune system and bone. Immunol Rev 2005;208:50-65.

30. Koga T, Inui M, Inoue K, Kim S, Suematsu A, Kobayashi E, et al. Costimulatory signals mediated by the ITAM motif cooperate with RANKL for bone homeostasis. Nature 2004;428(6984):758-63.

31. Zou W, Kitaura H, Reeve J, Long F, Tybulewicz VL, Shattil SJ, et al. Syk, c-Src, the alphavbeta3 integrin, and ITAM immunoreceptors, in concert, regulate osteoclastic bone resorption. J Cell Biol 2007;176(6):877-88.

32. Mocsai A, Humphrey MB, Van Ziffle JA, Hu Y, Burghardt A, Spusta SC, et al. The immunomodulatory adapter proteins DAP12 and Fc receptor gamma-chain (FcRgamma) regulate development of functional osteoclasts through the Syk tyrosine kinase. Proc Natl Acad Sci U S A 2004;101(16):6158-63.

33. Cella M, Buonsanti C, Strader C, Kondo T, Salmaggi A, Colonna M. Impaired differentiation of osteoclasts in TREM-2-deficient individuals. J Exp Med 2003;198(4):645-51. 
34. Paloneva J, Mandelin J, Kiialainen A, Bohling T, Prudlo J, Hakola P, et al. DAP12/TREM2 deficiency results in impaired osteoclast differentiation and osteoporotic features. J Exp Med 2003;198(4):669-75.

35. Takayanagi H, Ogasawara K, Hida S, Chiba T, Murata S, Sato K, et al. T-cell-mediated regulation of osteoclastogenesis by signalling cross-talk between RANKL and IFN-gamma. Nature 2000;408(6812):600-5.

36. Takayanagi H, Kim S, Matsuo K, Suzuki H, Suzuki T, Sato K, et al. RANKL maintains bone homeostasis through c-Fos-dependent induction of interferon-beta. Nature 2002;416(6882):744-9.

37. Shioi A, Teitelbaum SL, Ross FP, Welgus HG, Suzuki H, Ohara J, et al. Interleukin 4 inhibits murine osteoclast formation in vitro. J Cell Biochem 1991;47(3):272-7.

38. Duplomb L, Baud'huin M, Charrier C, Berreur M, Trichet V, Blanchard F, et al. Interleukin-6 inhibits receptor activator of nuclear factor kappaB ligand-induced osteoclastogenesis by diverting cells into the macrophage lineage: key role of Serine 727 phosphorylation of signal transducer and activator of transcription 3. Endocrinology 2008;149(7):3688-97.

39. Xu LX, Kukita T, Kukita A, Otsuka T, Niho Y, Iijima T. Interleukin-10 selectively inhibits osteoclastogenesis by inhibiting differentiation of osteoclast progenitors into preosteoclast-like cells in rat bone marrow culture system. J Cell Physiol 1995;165(3):624-9. 40. Chen L, Wei XQ, Evans B, Jiang W, Aeschlimann D. IL-23 promotes osteoclast formation by up-regulation of receptor activator of NF-kappaB (RANK) expression in myeloid precursor cells. Eur J Immunol 2008;38(10):2845-54.

41. Kamiya S, Nakamura C, Fukawa T, Ono K, Ohwaki T, Yoshimoto T, et al. Effects of IL23 and IL-27 on osteoblasts and osteoclasts: inhibitory effects on osteoclast differentiation. J Bone Miner Metab 2007;25(5):277-85.

42. Takayanagi H, Sato K, Takaoka A, Taniguchi T. Interplay between interferon and other cytokine systems in bone metabolism. Immunol Rev 2005;208:181-93.

43. Sorensen MG, Henriksen K, Schaller S, Henriksen DB, Nielsen FC, Dziegiel MH, et al. Characterization of osteoclasts derived from CD14+ monocytes isolated from peripheral blood. J Bone Miner Metab 2007;25(1):36-45.

44. Takayanagi H. The role of NFAT in osteoclast formation. Ann N Y Acad Sci 2007; 1116:227-37.

45. Aliprantis AO, Ueki Y, Sulyanto R, Park A, Sigrist KS, Sharma SM, et al. NFATc1 in mice represses osteoprotegerin during osteoclastogenesis and dissociates systemic osteopenia from inflammation in cherubism. J Clin Invest 2008;118(11):3775-89.

46. Wagner EF, Eferl R. Fos/AP-1 proteins in bone and the immune system. Immunol Rev 2005;208:126-40.

47. Takayanagi H. Mechanistic insight into osteoclast differentiation in osteoimmunology. $\mathrm{J}$ Mol Med 2005;83(3):170-9.

48. Colonna M, Turnbull I, Klesney-Tait J. The enigmatic function of TREM-2 in osteoclastogenesis. Adv Exp Med Biol 2007;602:97-105.

49. Sato K, Suematsu A, Okamoto K, Yamaguchi A, Morishita Y, Kadono Y, et al. Th17 functions as an osteoclastogenic helper $\mathrm{T}$ cell subset that links $\mathrm{T}$ cell activation and bone destruction. J Exp Med 2006;203(12):2673-82. 
50. Stumhofer JS, Laurence A, Wilson EH, Huang E, Tato CM, Johnson LM, et al. Interleukin 27 negatively regulates the development of interleukin 17-producing $\mathrm{T}$ helper cells during chronic inflammation of the central nervous system. Nat Immunol 2006;7(9):937-45. 


\section{Figure Legends}

\section{Figure 1}

IL-27 inhibits human osteoclastogenesis in a dose- and time-dependent manner.

Freshly isolated human CD14+ cells were cultured with MCSF (20ng/ml) for 48h and RANKL (40ng/ml) was added on Day 3 as described in Materials and Methods. IL-27 (3, 10, 30 and $100 \mathrm{ng} / \mathrm{ml}$ ) was added at the initiation of cultures (Day1) or later (Day 3 or Day 5). (A and B), TRAP positive multinucleated ( $>3$ nuclei) cells were counted 5 days after RANKL addition and representative data of one out of three independent experiments are shown as mean \pm SD from triplicate wells of 96 well plates $(*=p>0.05, * *=p<0.05$ and $* * *=p<0.01 . p$ values were calculated by Student's $t$ test). C, Cathepsin K mRNA was measured by using real-time PCR and normalized relative to GAPDH expression. The means \pm SD of triplicate determinants in a representative experiment of three independent experiments are shown; small SDs are not readily apparent because of large inductions. D (left panels), freshly isolated human monocytes were cultured as in A and osteoclast function was measured by a resorption pit formation assay. D (right panel), Human CD14 $4^{+}$monocytes were cultured with MCSF $(20 \mathrm{ng} / \mathrm{ml})$ in the presence or absence of IL-27 (100ng/ml) for 2days. Cell viability was measured by MTT assay. Representative results of at least three independent experiments are shown.

\section{Figure 2}

IL-27 abrogates RANKL-mediated induction of NFATc1.

A and B, Freshly isolated human CD14+ cells were cultured with MCSF (20ng/ml) in the presence or absence of IL-27 (100ng/ml) for $48 \mathrm{~h}$ and then were stimulated with RANKL. C and D, Freshly isolated human CD14+ cells were cultured with MCSF (20ng/ml) for $48 \mathrm{~h}$ and then 
were stimulated with RANKL (40ng/ml) in the presence or absence of IL-27 (100ng/ml).

NFATc1 protein expression 24, 48 and $72 \mathrm{~h}$ following RANKL stimulation was measured by immunoblotting (A and C). NFATc1 mRNA was measured using real-time PCR and normalized relative to GAPDH expression (B and D). Representative results of at least three independent experiments are shown.

\section{Figure 3}

IL-27 inhibits RANKL-mediated activation of MAPK and NF- $\kappa$ B pathways and induction of cJun.

Freshly isolated human CD14+ cells were cultured with MCSF (20ng/ml) in the presence or absence of IL-27 (100ng/ml) for 48h. Control and IL27-treated cells were stimulated with RANKL (40ng/ml) for 5, 10 and 15 minutes (A and B) or for 0.5, 1 and $3 \mathrm{~h}$ (C). Immunoblotting was used to measure threonine 202/tyrosine 204 phosphorylation of Erk1/Erk2 and threonine

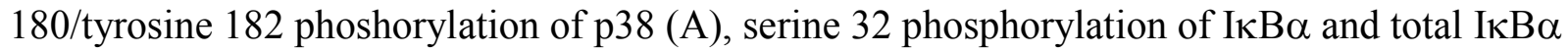
(B), total TRAF6 (B), and nuclear c-Jun and TBP proteins (C). Representative results of at least

five independent experiments are shown. D, RANK mRNA was measured in control and IL-27treated cells using real-time PCR and normalized relative to GAPDH expression. The means \pm SD of triplicate determinants in a representative experiment of three independent experiments are shown; small SDs are not readily apparent because of large inductions.

\section{Figure 4}

IL-27 inhibits TREM-2 expression. 
Freshly isolated human CD14+ cells were cultured with MCSF $(20 \mathrm{ng} / \mathrm{ml})$ in the presence or absence of IL-27 (100ng/ml) for 24 and 48h. TREM-2 mRNA (A), OSCAR mRNA, SIRP $\beta 1$ mRNA and ILT7 mRNA (B) and DAP12 and FcR $\gamma$ mRNA (C) was measured using real-time PCR and normalized relative to GAPDH expression. Representative results of at least four independent experiments are shown.

\section{Figure 5}

Synovial fluid macrophages derived from Rheumatoid Arthritis patients are refractory to IL-27.

Freshly isolated $\mathrm{CD} 14^{+}$cells derived from synovial fluid of five RA patients were stimulated for $15 \mathrm{~min}(\mathrm{~A})$ or $3 \mathrm{~h}(\mathrm{~B})$ with hIFN- $\gamma(100 \mathrm{U} / \mathrm{ml})$ or hIL-27 (100ng/ml). A, tyrosine 701 phosphorylation of STAT1 was measured by immunoblotting. Representative results of one out of five independent experiments are shown. B, CXCL10 mRNA expression was measured using real-time PCR and normalized relative to GAPDH expression ( $\mathrm{p}<0.001$ by paired Student's $t$ test, $\mathrm{n}=5$ ).

\section{Figure 6}

IL-27 is a moderate inhibitor of in vitro osteoclastogenesis in murine systems.

A, Murine monocytes were stimulated for 5, 10 and 20min with $\mathrm{mIFN}-\gamma(100 \mathrm{U} / \mathrm{ml})$ or $\mathrm{mIL}-27$ (100ng/ml). Immunoblotting was used to measure tyrosine 701 phosphorylation of STAT1 and tyrosine 705 phosphorylation of STAT3. (B-C) Bone marrow-derived osteoclast precursors obtained from C57BL/6J mice were cultured in the presence of MCSF $(20 \mathrm{ng} / \mathrm{ml})$ and RANKL (80ng/ml) was added as described in Materials and Methods. IL-27 (1, 10 and 100ng/ml) was 
added 1 day before RANKL (Pretreatment) or simultaneously with RANKL. (B and C), TRAP positive multinucleated ( $>3$ nuclei) cells were counted 5 days after RANKL addition and representative data of one out of three independent experiments are shown as mean $\pm \mathrm{SD}$ from triplicate wells of 96 well plates $(*=p>0.05$ and $* *=p<0.05$. p values were calculated by Student's $t$ test). D, The expression of WSX-1 m-RNA was measured by PCR in freshly isolated human CD14+ and CD14- cells and in murine BMDM and splenocytes. 
A

RANKL

IL-27(ng/ml): 0

Day:

B

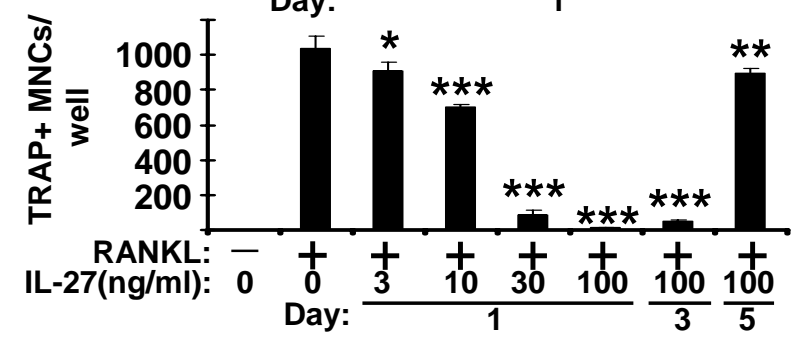

$10 \quad 30 \quad 100-\frac{100}{3} \frac{100}{5}$
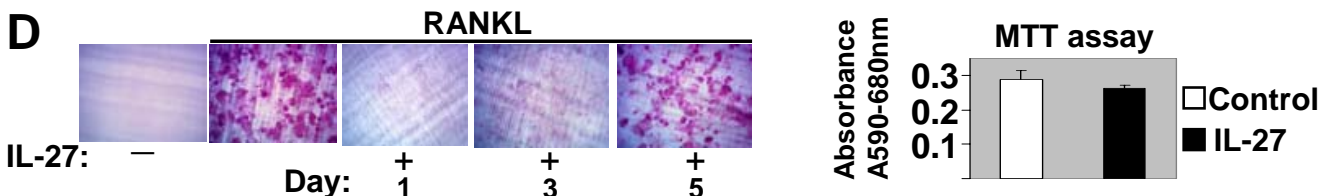

Figure 1. IL-27 inhibits human osteoclastogenesis in a dose- and time-dependent manner. Freshly isolated human CD14+ cells were cultured with MCSF $(20 \mathrm{ng} / \mathrm{ml})$ for $48 \mathrm{~h}$ and RANKL $(40 \mathrm{ng} / \mathrm{ml})$ was added on Day 3 as described in Materials and Methods. IL-27 (3, 10, 30 and 100ng/ml) was added at the initiation of cultures (Day1) or later (Day 3 or Day 5). (A and B), TRAP positive multinucleated (> 3 nuclei) cells were counted 5 days after RANKL addition and representative data of one out of three independent experiments are shown as mean \pm SD from triplicate wells of 96 well plates $(*=p>0.05$, $* *=\mathrm{p}<0.05$ and $* * *=\mathrm{p}<0.01$. p values were calculated by Student's $t$ test). C, Cathepsin K mRNA was measured by using real-time PCR and normalized relative to GAPDH expression. The means \pm SD of triplicate determinants in a representative experiment of three independent experiments are shown; small SDs are not readily apparent because of large inductions. D (left panels), freshly isolated human monocytes were cultured as in A and osteoclast function was measured by a resorption pit formation assay. D (right panel), Human CD14+ monocytes were cultured with MCSF (20ng/ml) in the presence or absence of IL-27 (100ng/ml) for 2days. Cell viability was measured by MTT assay. Representative results of at least three independent experiments are shown. 


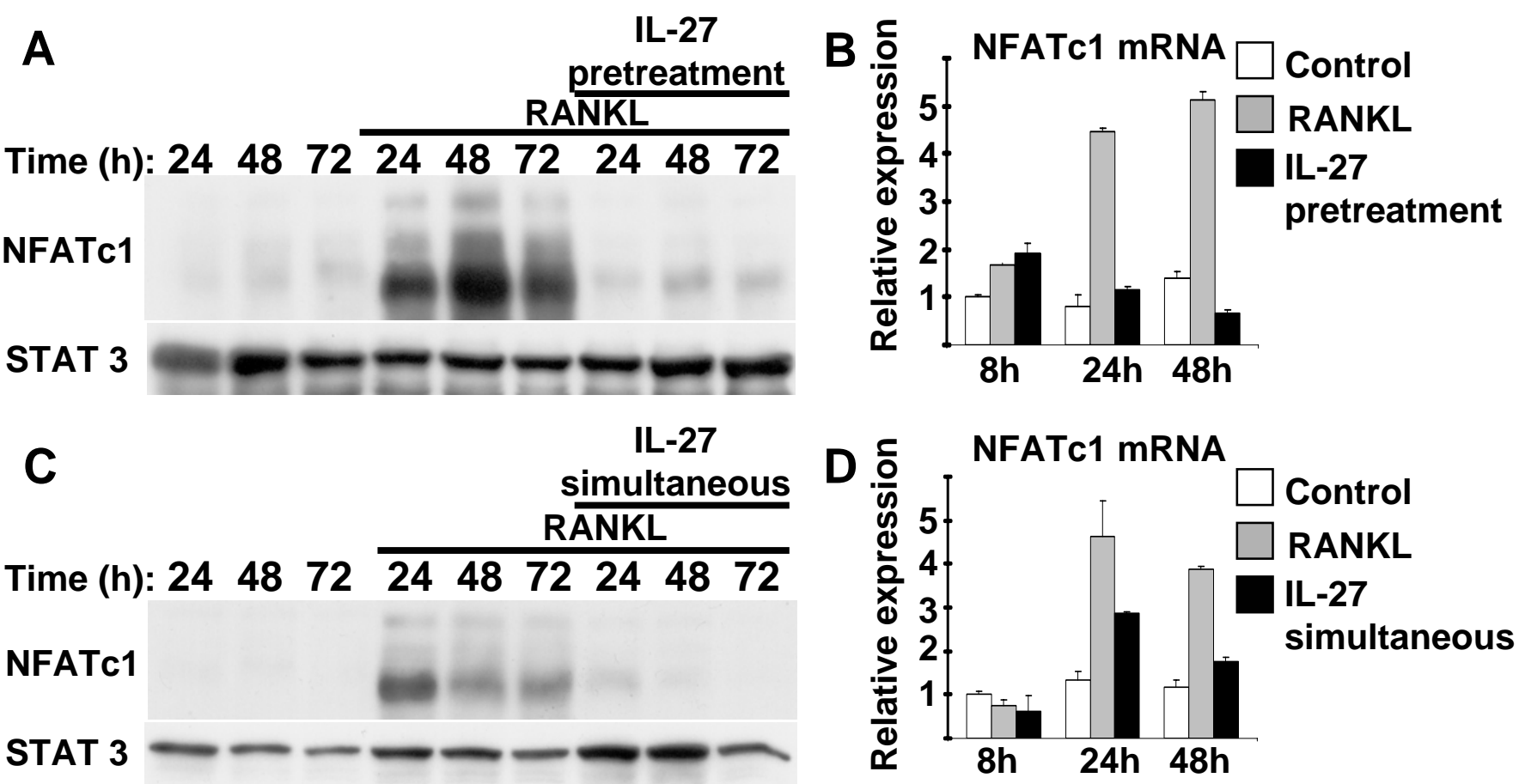

Figure 2. IL-27 abrogates RANKL-mediated induction of NFATc1. A and B, Freshly isolated human CD14+ cells were cultured with MCSF (20ng/ml) in the presence or absence of IL-27 (100ng/ml) for 48h and then were stimulated with RANKL. C and D, Freshly isolated human CD14+ cells were cultured with MCSF $(20 \mathrm{ng} / \mathrm{ml})$ for $48 \mathrm{~h}$ and then were stimulated with RANKL $(40 \mathrm{ng} / \mathrm{ml})$ in the presence or absence of IL-27 $(100 \mathrm{ng} / \mathrm{ml})$. NFATc1 protein expression 24, 48 and 72h following RANKL stimulation was measured by immunoblotting (A and C). NFATc1 mRNA was measured using real-time

PCR and normalized relative to GAPDH expression (B and D). Representative results of at least three independent experiments are shown. 
A

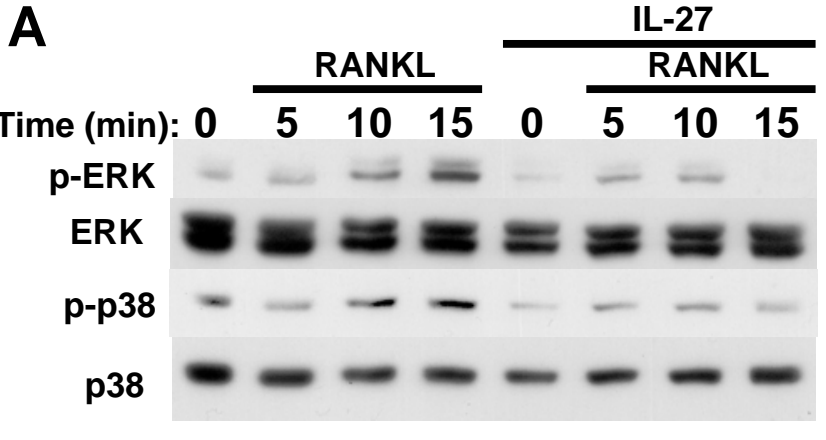

B

IL-27

C

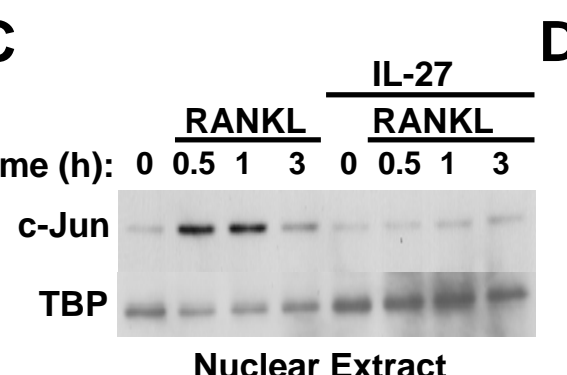

D

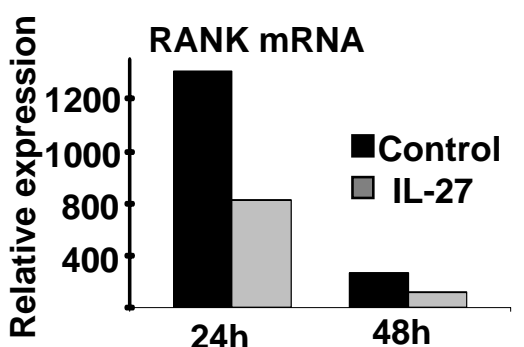

Figure 3. IL-27 inhibits RANKL-mediated activation of MAPK and NF- $\kappa$ B pathways and induction of c-Jun. Freshly isolated human CD14+ cells were cultured with MCSF $(20 \mathrm{ng} / \mathrm{ml})$ in the presence or absence of IL-27 $(100 \mathrm{ng} / \mathrm{ml})$ for $48 \mathrm{~h}$. Control and IL27-treated cells were stimulated with RANKL (40ng/ml) for 5, 10 and 15 minutes (A and B) or for 0.5, 1 and 3h (C). Immunoblotting was used to measure threonine 202/tyrosine 204 phosphorylation

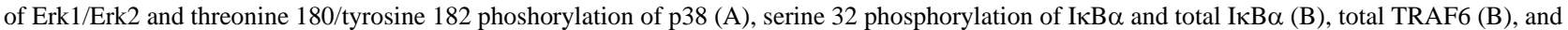
nuclear c-Jun and TBP proteins (C). Representative results of at least five independent experiments are shown. D, RANK mRNA was measured in control and IL-27-treated cells using real-time PCR and normalized relative to GAPDH expression. The means \pm SD of triplicate determinants in a

representative experiment of three independent experiments are shown; small SDs are not readily apparent because of large inductions. 

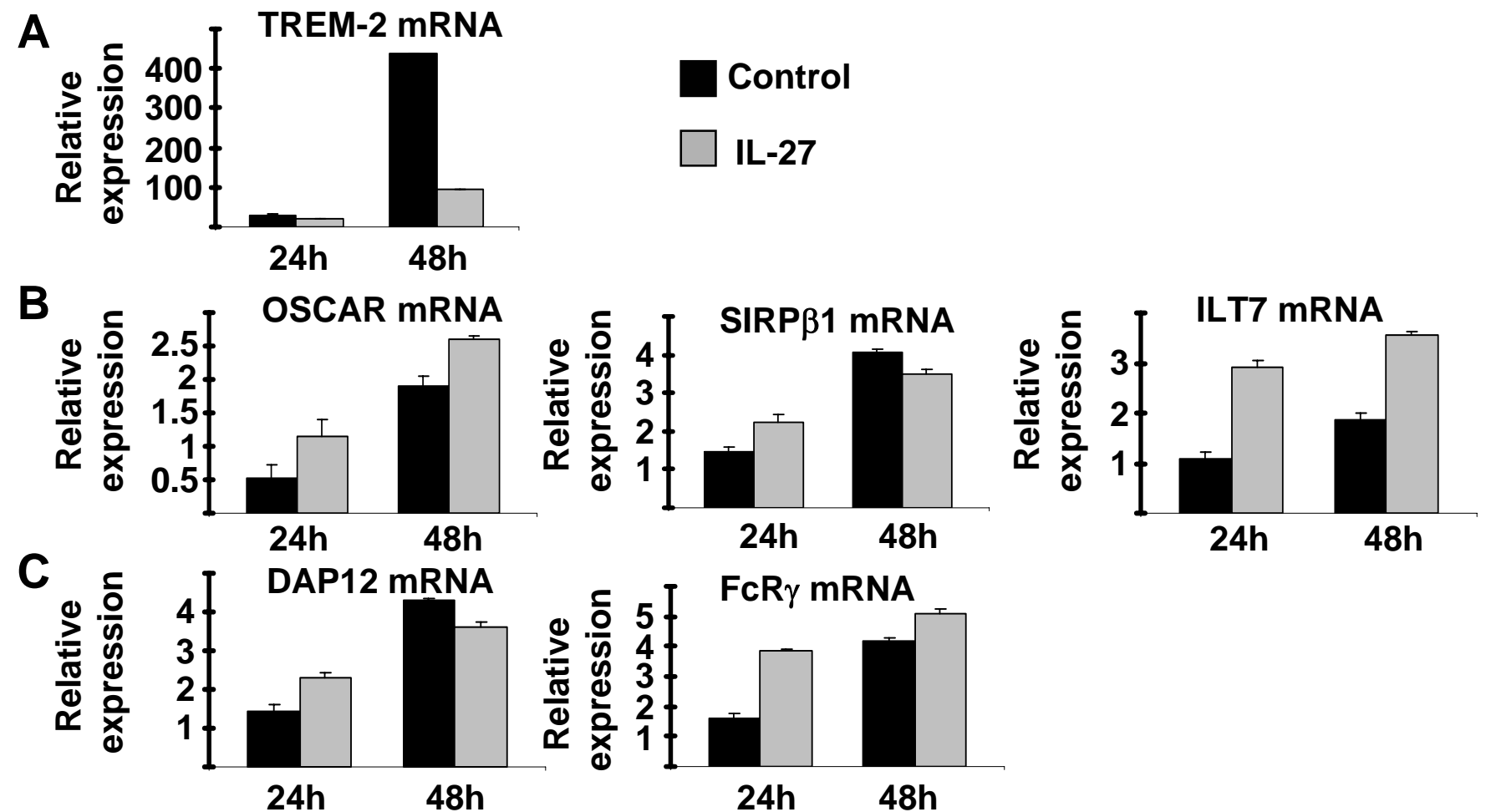

Figure 4. IL-27 inhibits TREM-2 expression. Freshly isolated human CD14+ cells were cultured with MCSF (20ng/ml) in the presence or absence of IL-27 (100ng/ml) for 24 and 48h. TREM-2 mRNA (A), OSCAR mRNA, SIRP $\beta 1$ mRNA and ILT7 mRNA (B) and DAP12 and FcR $\gamma$ mRNA (C) was measured

using real-time PCR and normalized relative to GAPDH expression. Representative results of at least four independent experiments are shown. 
A

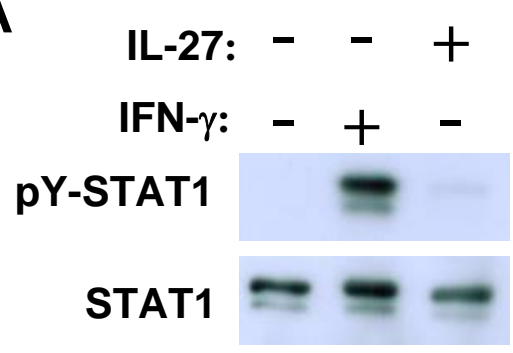

RA patient \#1
B

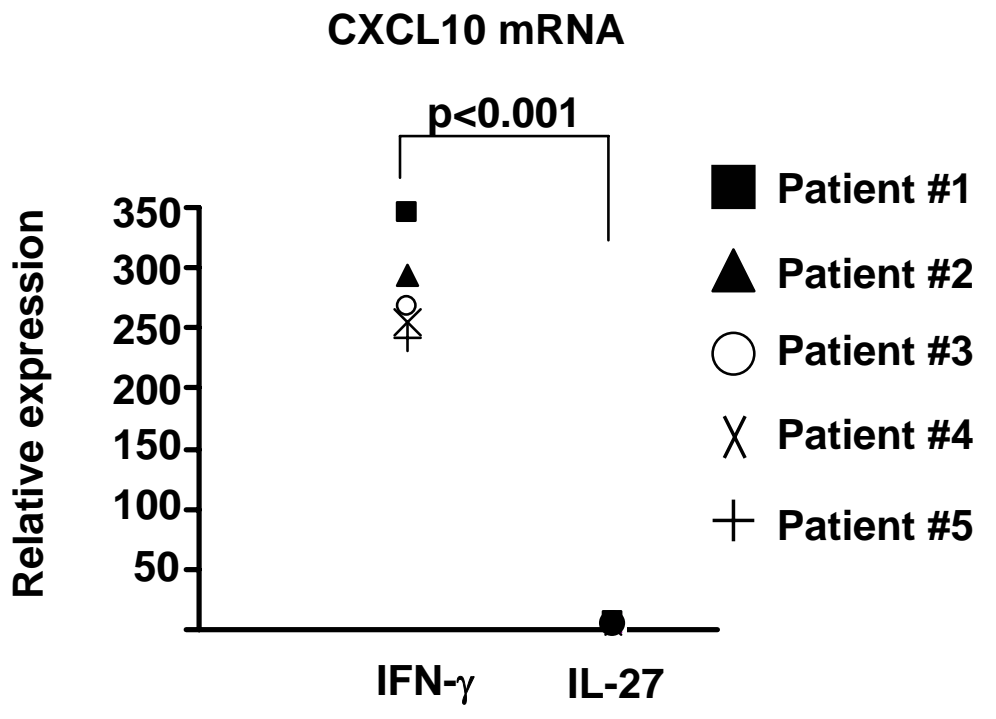

Figure 5. Synovial fluid macrophages derived from Rheumatoid Arthritis patients are refractory to IL-27. Freshly isolated CD14+ cells derived from synovial fluid of five RA patients were stimulated for $15 \mathrm{~min}$ (A) or 3h (B) with hIFN- $\gamma(100 \mathrm{U} / \mathrm{ml})$ or hIL-27 (100ng/ml). A, tyrosine 701 phosphorylation of STAT1 was measured by immunoblotting. Representative results of one out of five independent experiments are shown. B, CXCL10 mRNA expression was measured using real-time PCR and normalized relative to GAPDH expression ( $p<0.001$ by paired Student's $t$ test, $\mathrm{n}=5)$. 
A
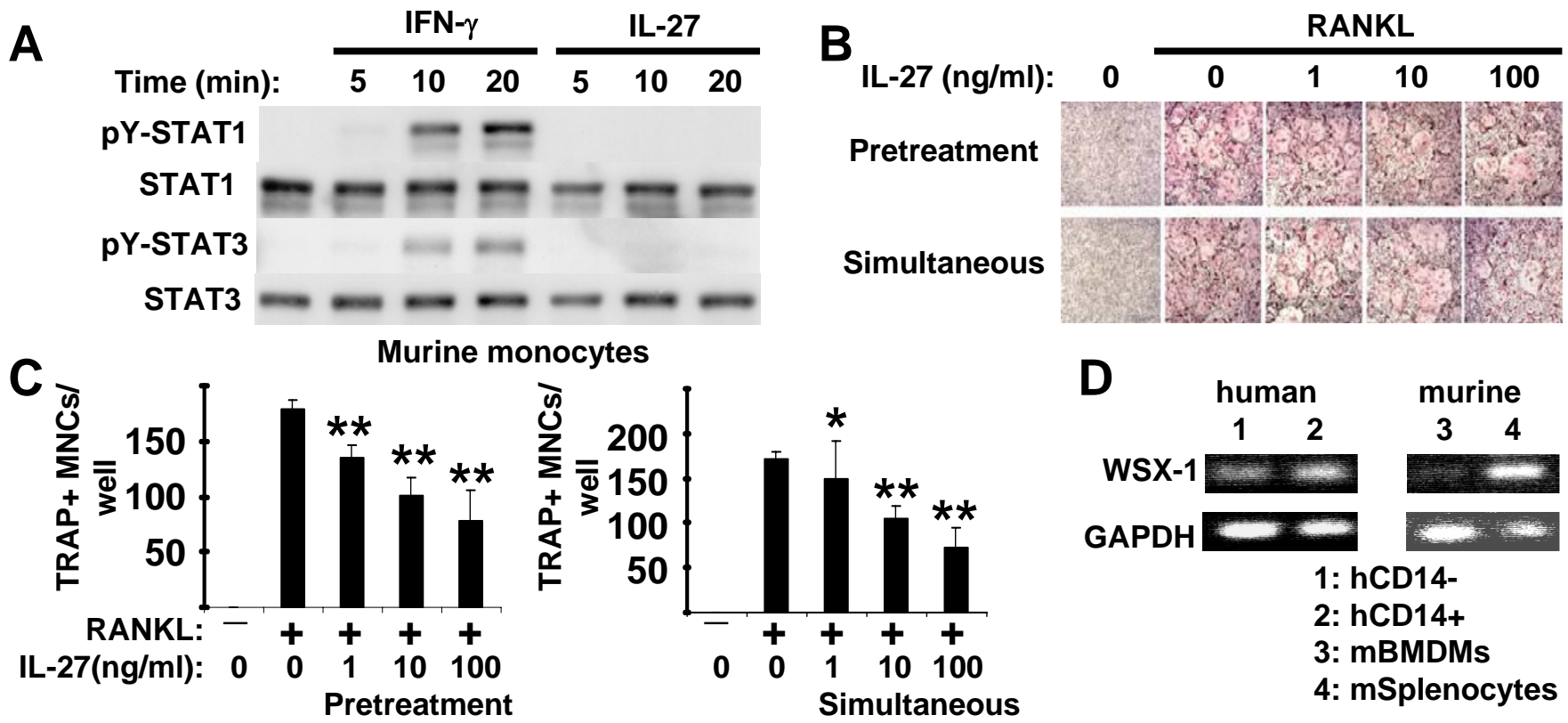

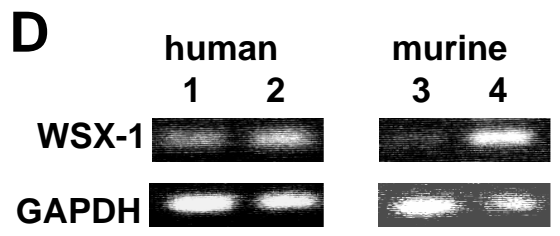

1: hCD14-

2: hCD14+

3: mBMDMs

4: mSplenocytes

Figure 6. IL-27 is a moderate inhibitor of in vitro osteoclastogenesis in murine systems. A, Murine monocytes were stimulated for 5, 10 and 20 min with mIFN- $\gamma(100 \mathrm{U} / \mathrm{ml})$ or $\mathrm{mIL}-27(100 \mathrm{ng} / \mathrm{ml})$. Immunoblotting was used to measure tyrosine 701 phosphorylation of STAT1 and tyrosine 705 phosphorylation of STAT3. (B-C) Bone marrow-derived osteoclast precursors obtained from C57BL/6J mice were cultured in the presence of MCSF (20ng/ml) and RANKL (80ng/ml) was added as described in Materials and Methods. IL-27 (1, 10 and 100ng/ml) was added 1 day before RANKL (Pretreatment) or simultaneously with RANKL. (B and C), TRAP positive multinucleated ( $>3$ nuclei) cells were counted 5 days after RANKL addition and representative data of one out of three independent experiments are shown as mean \pm SD from triplicate wells of 96 well plates $(*=p>0.05$ and $* *=p<0.05$. $p$ values were calculated by Student's $t$ test). D, The expression of WSX-1 m-RNA was measured by PCR in freshly isolated human CD14+ and CD14- cells and in murine BMDM and splenocytes. 